\title{
Higher Lefschetz invariants for foliated manifolds
}

\author{
Dissertation \\ zur Erlangung des mathematisch-naturwissenschaflichen \\ Doktorgrades \\ "Doctor rerum naturalium" \\ der Georg-August-Universität Göttingen \\ vorgelegt
}

\author{
von \\ Alessandro Fermi \\ aus Abbiategrasso (Mailand)
}

Göttingen 2012 
1. Gutachter: Prof. Thomas Schick

2. Gutachter: Prof. Ralf Meyer

3. Gutachter: Prof. Paolo Piazza

Tag der mündlichen Prüfung: 


\section{Acknowledgments}

There are many people who contributed directly to the writing of this work and whom I would like to thank heartly.

First, let me thank my supervisor Thomas Schick for his patience during these years, for all the discussions we had and for the freedom he accorded to me to develop my own research.

I want to thank Paolo Piazza for many helpful discussions and valuable suggestions about index theory, which made me better understand this enchanting subject.

Let me thank Ralf Meyer for his comments and suggestions, that led to an improvement of the work.

I want to thank J. Heitsch, M.T. Benameur, S. Hurder, G. Skandalis, C. Voigt and P. Carrillo-Rouse for helpful suggestions and correspondence.

A special thank goes to everyone, who participated in the many informal students seminars we organized. I really appreciated the scientific atmosphere we created and I am indebted to all these people.

I would like to thank heartly Alessandro Valentino and Sara Azzali for the great time we had together in Goettingen, discussing not only about maths and science, but also about politics, economics, culture and life in general.

I want to thank Paolo Antonini, for all the discussions we had and all the ideas we discussed together.

A really special thanks goes to Iakovos Androulidakis, for his help and encouragement, all the ideas he shared with me and for his great humanity.

I am particularly indebted to my parents, Marco e Rita, without whom this work would not have been possible. They let me do all the steps I wanted to do in my life, stepping aside when the things went well, and helping me, when the things did not go so well.

Finally, I want to thank heartly Francesca and I want to dedicate to her the following words

"Cuando no puedo mirar tu cara miro tus pies.

Tus pies de hueso arqueado, tus pequeños pies duros. 
Yo sé que te sostienen,

y que tu dulce peso

sobre ellos se levanta.

Tu cintura y tus pechos,

la duplicada púrpura de tus pezones,

la caja de tus ojos que recién han volado,

tu ancha boca de fruta,

tu cabellera roja,

pequeña torre mía.

Pero no amo tus pies

sino porque anduvieron

sobre la tierra y sobre

el viento y sobre el agua,

hasta que me encontraron."

(Tus pies, Pablo Neruda)

Cianfri, thank you for everything! 


\section{Contents}

\begin{tabular}{lll}
\hline & Foliations & 8
\end{tabular}

1.1 Basic definitions and some examples . . . . . . . . . . . . 8

1.2 Groups of diffeomorphisms preserving the foliation . . . . . . . 15

1.3 The leaf space and the holonomy groupoid . . . . . . . . . . 20

1.4 Holonomy groupoid of a foliated $\Gamma$-manifold $\ldots \ldots \ldots \ldots$

1.5 Lie $\Gamma$-groupoids and inner automorphisms . . . . . . . . . . 40

2 Foliation $\mathrm{C}^{*}$-algebras, Operators and equivariant Index classes $\mathbf{5 0}$

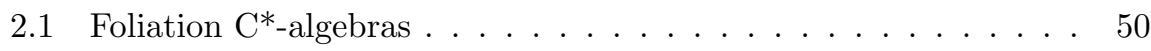

2.1.1 The smooth convolution algebra associated to a Lie groupoid 50

2.1.2 The reduced $\mathrm{C}^{*}$-algebras associated to a Lie groupoid . . 52

$2.1 .3 \quad$ Automorphisms defined by a group $\Gamma \ldots \ldots \ldots 7$

$2.2 \quad$ Equivariant K-theory and the equivariant analytic index class . . 63

$2.2 .1 \quad$ Equivariant $K$-theory $\ldots \ldots \ldots \ldots \ldots$

2.2 .2 Pseudo-differential operators, ellipticity and the funda-

$2.2 .3 \quad$ The equivariant index class and the Lefschetz class . . . . 73

2.3 Pseudo-differential calculus for foliated bundles . . . . . . . . . 79

\begin{tabular}{|lll}
3 & Equivariant and Twisted Cyclic Cohomology & 82
\end{tabular}

3.1 Equivariant Cyclic Cohomology for unital $\Gamma$-algebras . . . . . . . 82

$3.1 .1 \quad$ Cyclic group action . . . . . . . . . . . . . . . 84

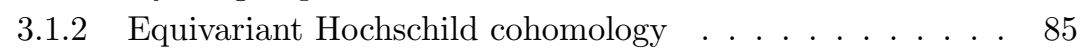

$3.1 .3 \quad$ Equivariant cyclic cohomology $\ldots \ldots \ldots$. . . . . . . . . 86

$3.2 \quad$ Equivariant Cyclic Bicomplex _ . . . . . . . . . . . . . . . 88

3.3 The equivariant SBI-sequence and the periodic theory . . . . . . 91

3.3 .1 Some Remarks and construction . . . . . . . . . . . . 93

3.4 The pairing with equivariant K-theory . . . . . . . . . . . . . . 98

3.5 Twisted cyclic cohomology and the pairing with equivariant $\mathrm{K}$ -

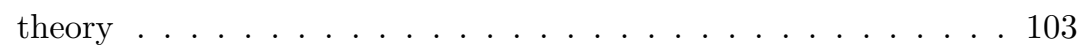

4 Equivariant Cyclic Cocycles for foliated manifolds 110

4.1 The equivariant transverse fundamental class . . . . . . . . . . . . 111

$4.1 .1 \quad$ Haefliger-type transverse cohomologies . . . . . . . . . . 111

$4.1 .2 \quad$ Cyclic cocycles on the twisted holonomy groupoid . . . . 113 
4.1 .3 Equivariant cyclic cocycles . . . . . . . . . . . . . . . 120

$4.1 .4 \quad$ Twisted cyclic cocycles. . . . . . . . . . . . . . . . . . 128

4.2 The equivariant Godbillon-Vey cyclic class . . . . . . . . . . . . 129

4.2 .1 The Godbillon-Vey class: classical definition . . . . . . . . 129

4.2 .2 The Godbillon-Vey class: the Moriyoshi-Natsume' cyclic

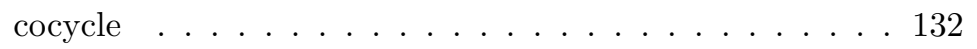

$4.2 .3 \quad$ The Godbillon-Vey class: the general case . . . . . . . . . 143

$4.2 .4 \quad$ The equivariant Godbillon-Vey cyclic cocycle . . . . . . . 155

4.3 Some examples and further developments $\ldots \ldots \ldots$

\begin{tabular}{ll|l|l|l|l|}
\hline A Proof of Lemma & 165
\end{tabular} 


\section{Introduction}

A regular foliation of a smooth manifold is, roughly speaking, a partition into immersed submanifolds, called the leaves of the foliation. These submanifolds are supposed to be of the same dimension and to fit together in a regular way. Foliations of manifolds occur in various situations. For instance, as the fibers of a submersion, as the orbits of a Lie group action on a smooth manifold or as solutions of differential equations and integrable systems (cf. [11, [12]).

The origin of this notion can be traced back to the works of Ehresmann, Reeb, Haefliger and many others in the ' 50 s, who were motivated by the problem of existence of completely integrable vector fields on 3-manifolds. They started to investigate these objects sistematically and to establish their first properties. A feature, that appeared immediately, is the "singular" nature of these geometrical objects. A foliated manifold can not be in general viewed as the total space of a fibration with leaves the fibers, since even in the simplest examples the base space is a space with trivial topology. Moreover, the leaves of a foliation need not be all diffemorphic to each other and the way in which they are immersed in the ambient manifold is generally very complicated and presents a strange behaviour with respect to the topology of the ambient manifold. We refer, for instance, to [39] and [11] for examples and further comments.

Put in a nutshell, foliated manifolds have a singular "transverse structure" that encodes geometric and dynamical properties of the foliation, but that is hard to study by means of usual methods of algebraic topology and differential geometry.

Thus, people working on foliations were forced to think of them as a class of "singular spaces" and were led to the idea of describing the transverse structure of a foliated manifold through a groupoid. This turned out to be a very profound idea and a unifying concept for dealing with other classes of singular spaces, like orbit spaces of smooth group actions on manifolds.

To a foliated manifold $(M, \mathcal{F})$ one can associate various groupoids, each of which reflects some property of the foliation and of its transverse geometry. Among them, there is the holonomy groupoid $H(\mathcal{F})$, the smallest Lie groupoid associated to the foliation which unwraps simultaneously all leaves. It was first rigorously defined in [47, following ideas of Ehresmann, and later it has been extensively studied form different points of view.

In the early ' 80 s Connes started in [13] to investigate the analytical significance of the holonomy groupoid $H(\mathcal{F})$. Using it, he was able to associate to any foli- 
ation $(M, \mathcal{F})$ various operator algebras, which ought to describe the transverse geometry of the foliation. Indeed, in [13], 14] and [16] he defined a von Neumann algebra $W^{*}(M, \mathcal{F})$, which should reflect measure theoretical properties of the transverse structure of $\mathcal{F}$, a $C^{*}$-algebra $C^{*}(M, \mathcal{F})$, which should encode the topological features of $(M, \mathcal{F})$ and suitable subalgebras of this $C^{*}$-algebra, which ought to codify differential-topological properties of the foliation.

The above algebras are no longer commutative, but in general highly noncommutative, showing from another point of view the singular nature of the transverse structure of the foliation.

Furthermore, using powerful invariants of such algebras, like Kasparov's bivariant $K$-theory (see [9]) and cyclic-type cohomology theories (cf. [17], Chapter III, 37] or [18]), he and his collaborators have succeeded in generalizing many aspects of index theory for compact manifolds to the more general context of compact foliated manifolds.

Let us be a bit more precise. Given a compact foliated manifold $(M, \mathcal{F})$, complex vector bundles $E$ and $F$ over $M$ and a leafwise elliptic differential operator $D: C^{\infty}(M, E) \rightarrow C^{\infty}(M, F)$ one can associate to $D$ an element of the $K$-theory $K_{0}\left(C^{*}(M, \mathcal{F})\right)$ of the foliation $C^{*}$-algebra $C^{*}(M, \mathcal{F})$. This element is called the analytic index class of $D$ and is denoted by $\operatorname{ind}_{a}(D)$.

Using Kasparov's bivariant $K$-theory, one can show that $i n d_{a}(D)$ depends only on topological data of the foliated manifold $(M, \mathcal{F})$. This is the content of the celebrated Connes-Skandalis longitudinal index theorem for foliations ([17], Chapter II, Section 9. $\gamma$ and the references therein). However, this theorem is abstract and does not provide explicit formulas for $i n d_{a}(D)$, relating the analytical index of $D$ with topological invariants of $(M, \mathcal{F})$.

To get such formulas, a crucial role is played by cyclic cocycles and their pairing with $K$-theory (see [15] or [17, Chapter III, Section 1 and Section 3). In general, any cyclic cocycle on an algebra $\mathcal{A}$ determines a complex-valued additive map on the $K$-theory $K_{0}(\mathcal{A})$ and one can attempt to compute explicitly the values of suitable $K$-theory classes under this map. In geometric situations, like those provided by foliations, this approach turns out to be very important and deep (cf. [16, [17, Chapter III). Thus, a fundamental problem of this method is that of constructing as many as possible "interesting" cyclic cocycles. In the case of a foliated manifold $(M, \mathcal{F})$, Connes [16], [17, Chapter III, constructs various geometric cyclic cocycles. Using these cocycles and the additive maps on $K$-theory induced by them, he gets explicit index formulae, evaluating these maps on the $K$-theory class $i n d_{a}(D)$. Surprisingly, the resulting index formulae involve very important invariants of the foliation, in particular, the so-called exotic characteristic classes of $(M, \mathcal{F})$ (see [12], Chapter 6 for the construction and properties). The most famous example is that of the Godbillon-Vey class and the corresponding Godbillon-Vey cyclic cocycle of a transversely oriented foliation of codimension 1 (cf. [16] and [4]).

These index formulae, relating on one side analytical invariants of the foliation and on the other side topological/geometric invariants, allow to prove deep results in the theory of foliated manifolds. Among them, we mention the in- 
variance of the $L$-class of a compact oriented and transversely oriented foliated manifold under orientation-preserving leafwise homotopy equivalences and the relationship between the nontriviality of the Godbillon-Vey class of a transversely oriented codimension 1 foliation $(M, \mathcal{F})$ and the type of the foliation von Neumann algebra $W^{*}(M, \mathcal{F})$. See [16] for details and further remarks.

An important variant of index theory is represented by the so-called equivariant index theory.

Classically, if $\Gamma$ is a compact Lie group acting smoothly on a compact manifold $M$ and $D$ is a $\Gamma$-equivariant elliptic operator on $M$, then it is interesting to take into account the action and to replace the numerical index of the operator $D$ by the natural representation of $\Gamma$ on $\operatorname{ker}(D)$ and on $\operatorname{coker}(D)$, i.e. the kernel and the cokernel of $D$, respectively. The resulting "character-index" is a natural generalization of the nonequivariant index and carries many interesting informations about the manifold and the action. The corresponding index theorem is a far-reaching generalization of the Lefschetz fixed point formula and has found many interesting applications in different branches of mathematics. We refer to [36] and the references therein for details and further remarks.

Considering what we have discussed above, it is natural to ask to which extent equivariant index theory can be generalized to the more general context of foliated manifolds.

First, one should make precise how a group acts on a foliated manifold. Given a foliated manifold $(M, \mathcal{F})$ let us assume to have a compact Lie group $\Gamma$ acting by foliation-preserving diffeomorphisms, i.e. for every $h \in \Gamma$ and every leaf $L$ of the foliation, $h(L)=L^{\prime}$, where $L^{\prime}$ is another leaf. Actually, we will concentrate on the case where each $h$ determines a diffeomorphism that maps each leaf into itself. A foliated manifold $(M, \mathcal{F})$ endowed with an action of a compact Lie group $\Gamma$ by leaf-preserving diffeomorphisms will be called a foliated $\Gamma$-manifold and denoted by $(M, \mathcal{F}, \Gamma)$. Such a triple will be our main object of study.

Now, if $(M, \mathcal{F}, \Gamma)$ is a foliated $\Gamma$-manifold, then the holonomy groupoid $H(\mathcal{F})$ of the foliation inherits an action of $\Gamma$, turning it into a so-called Lie $\Gamma$-groupoid. Using the holonomy groupoid $H(\mathcal{F})$ endowed with this $\Gamma$-action, Benameur [5] proved an equivariant version of the Connes-Skandalis index theorem in equivariant $K$-theory. More precisely, he proved that the equivariant index class $\operatorname{ind}_{a}^{\Gamma}(D) \in K_{0}^{\Gamma}\left(C^{*}(M, \mathcal{F})\right)$ of a leafwise elliptic $\Gamma$-equivariant differential operator on a foliated $\Gamma$-manifold $(M, \mathcal{F}, \Gamma)$ depends only on topological data. Moreover, generalizing methods of [3] to foliated manifolds, he is able to establish under some assumption a Lefschetz-type theorem for a foliated $\Gamma$-manifold $(M, \mathcal{F}, \Gamma)$. Roughly speaking, his theorem states that the Lefschetz class $L(h, D)$ of $h \in \Gamma$ with respect to a $\Gamma$-equivariant leafwise elliptic differential operator $D$, defined as a certain localization of $i n d_{a}^{\Gamma}(D)$, does only depend on topological data of the fixed point set $M^{h}$ of $h$ in $M$.

However, as in the nonequivariant case this result is very abstract and one is forced to develop other methods for extracting topological/geometric informations out of $L(h, D)$. In analogy with the nonequivariant theory a possible strategy is constructing equivariant cyclic cocycles on the foliated $\Gamma$-manifold, 
that determine additive maps on equivariant $K$-theory.

There are different way to define equivariant cyclic cocycles and equivariant cyclic (co-)homology. So far, the most general treatment to equivariant cyclic theories has been given by Voigt [46], where also many features of the theory, like stability and excission, are established. Unfortunately, for our purposes, his approach is difficult to deal with. Thus, we consider a different method to define equivariant cyclic cocycles (see [34, 23]), which seems to be more convenient for our applications. Roughly speaking, we shall view an equivariant cyclic cocycle as a $\Gamma$-equivariant "higher trace" with values in the set of continuous functions on $\Gamma$. With this definition, we will be able to show that an equivariant cyclic cocycle induces an additive map on equivariant $K$-theory.

Now, it turns out that in order to construct equivariant cyclic cocycles on a foliated $\Gamma$-manifold $(M, \mathcal{F}, \Gamma)$ as equivariant "higher traces", one has to understand the "transverse action" of the elements $h \in \Gamma$ on $(M, \mathcal{F})$. More precisely, since any element $h$ acts as a leaf-preserving diffeomorphism, then it determines a local diffeomorphism $\mathrm{Hol}(h)$ defined on a suitable local transversal $T_{x}$ passing through a point $x \in M$ to a suitable local transversal $T_{h(x)}$ through $h(x)$. If for all $x \in M$ these local transverse diffeomorphisms $\mathrm{Hol}(h)$ are holonomy transformations of the foliated manifold, there exists a smooth global bisection $\sigma_{h}$ of the holonomy groupoid $H(\mathcal{F})$ of the foliation that "lifts" the action of $h$ to the holonomy groupoid, i.e. $t \circ \sigma_{h}=h(t$ is the target map of $H(\mathcal{F}))$. If such a bisection exists for every $h \in \Gamma$, then we are able to construct equivariant cyclic cocycles on the holonomy groupoid $H(\mathcal{F})$ of $(M, \mathcal{F}, \Gamma)$.

Unfortunately, it is not true in general that for every leaf-preserving diffeomorphism $f$ there exists a smooth global bisection $\sigma_{f}$ of $H(\mathcal{F})$ with $t \circ \sigma_{f}=f$.

To overcome this problem, we introduce another Lie groupoid associated to the foliated $\Gamma$-manifold $(M, \mathcal{F}, \Gamma)$, that depends also on $\Gamma$ and that has a smooth global bisection $\sigma_{h}$ for every $h \in \Gamma$. This Lie groupoid, denoted by $H(M, \mathcal{F}, \Gamma)$, contains the holonomy groupoid $H(\mathcal{F})$ as open subgroupoid and "integrates" the same foliation $\mathcal{F}$ of $M$. Thus, it is a so-called foliation groupoid in the sense of [19]. Heuristically, $H(M, \mathcal{F}, \Gamma)$ encodes not only information of the transverse structure provided by holonomies, but also additional information coming from the local transverse diffeomorphisms $\mathrm{Hol}(h)$, for $h \in \Gamma$. Hence, it seems to be more appropriate than the holonomy groupoid for studying foliated $\Gamma-$ manifolds.

Using $H(M, \mathcal{F}, \Gamma)$, we will construct equivariant cyclic cocycles for $(M, \mathcal{F}, \Gamma)$. More precisely, generalizing first the construction of [7], we show that there exist equivariant cyclic cocycles coming from closed Haefliger currents of the foliation. Our construction is analogous to that in [7, but since we use the groupoid $H(M, \mathcal{F}, \Gamma)$ we can consider general leaf-preserving diffeomorphisms and not only holonomy diffeomorphisms, as in [7].

Furthermore, we will show that there exists an equivariant cyclic cocycle that corresponds to the Godbillon-Vey cyclic cocycle of the foliated manifold. To define such an equivariant cocycle, we first generalize the construction of the (nonequivariant) Godbillon-Vey cyclic cocycle of [4] to arbitrary transversely 
oriented foliations of codimension 1 . Then, we prove the existence of an equivariant cyclic cocycle, whose evaluation at the identity coincides with the GodbillonVey cyclic cocycle.

Through these equivariant cyclic cocycles we will define analytical higher Lefschetztype numbers associated to a foliated $\Gamma$-manifold $(M, \mathcal{F}, \Gamma)$ and to any leafwise elliptic $\Gamma$-equivariant differential operator $D$.

Let us now briefly explain how the text is organized. In the first chapter we begin with a section devoted to basic definitions and properties of foliated manifolds and of the associated group of diffeomorphisms preserving the foliation. In particular, we introduce the triples $(M, \mathcal{F}, \Gamma)$. In the subsequent section, we introduce the holonomy groupoid of the foliation, using the language of bisubmersions and bisections developed in [1]. Even though this approach may seem at first sight more complicated than the one using leafwise paths, it turns out to be more conceptual and more flexible for our purposes. Indeed, this is the language we use to construct the groupoid $H(M, \mathcal{F}, \Gamma)$ associated to a foliated $\Gamma$-manifold $(M, \mathcal{F}, \Gamma)$. We show that $H(M, \mathcal{F}, \Gamma)$ is a Lie groupoid "integrating" the foliation $\mathcal{F}$ of $M$ and containing the holonomy groupoid $H(\mathcal{F})$ as open Lie subgroupoid. In the last section, we present the notion of Lie $\Gamma$-groupoid and show that both groupoids $H(\mathcal{F})$ and $H(M, \mathcal{F}, \Gamma)$ inherite an action from the action of $\Gamma$ on $(M, \mathcal{F})$. The action of $\Gamma$ on $H(M, \mathcal{F}, \Gamma)$ is induced by smooth global bisections $\sigma_{h}$ of $H(M, \mathcal{F}, \Gamma)$, corresponding to the elements $h \in \Gamma$, and extends the $\Gamma$-action on $H(\mathcal{F})$.

In the second chapter we describe the algebras associated to a foliated manifold, that we will need in the following. More precisely, we introduce the smooth convolution algebra $C_{c}^{\infty}\left(G, \Omega^{1 / 2}\right)$ and the foliation $C^{*}$-algebra $C_{r}^{*}(G)$, where $G$ may denote both $H(\mathcal{F})$ and $H(M, \mathcal{F}, \Gamma)$. In the case of a foliated $\Gamma$-manifold, we will show that both algebras carry a natural action of $\Gamma$ by automorphisms, induced by the action of $\Gamma$ on $(M, \mathcal{F})$. Moreover, since the action of $\Gamma$ on $H(M, \mathcal{F}, \Gamma)$ is defined through bisections, we will prove that the $\Gamma$-action on $C_{r}^{*}(H(M, \mathcal{F}, \Gamma))$ is induced by suitable multipliers $S(h)$, for all $h \in \Gamma$. After this we survey some basic results of equivariant $K$-theory and we present the basic definitions and properties of $\Gamma$-equivariant pseudodifferential operators on a Lie groupoid endowed with an action of a group. Finally, we define $\Gamma$-equivariant index classes of an elliptic $\Gamma$-equivariant pseudodifferential operator $D$ and the corresponding Lefschetz classes of the conjugacy class $[h]$ of any element $h \in \Gamma$ with respect to $D$.

The third chapter contains basic definitions and properties of continuous equivariant cyclic cocycles and continuous equivariant cyclic cohomology of locally convex algebras. For technical reason, we define continuous equivariant cyclic cohomology only for Fréchet algebras. For such algebras, our treatment is based on the equivariant cyclic bicomplex, which provides a natural framework for nonunital algebras. Again, we point out that there is a different approach to equivariant cyclic cohomology developed in [46] that allow to define the theory for more general algebras.

However, even though the definition of continuous equivariant cyclic cohomology 
is well-posed only for a particular class of locally convex algebras, the notion of equivariant cyclic cocycle makes sense for any locally convex algebra and we show that any such equivariant cocycle gives rise to an additive map on $\Gamma$-equivariant $K$-theory with values in the central continuous functions of $\Gamma$. This is an important point in the theory, since it allows to define higher Lefschetz numbers on foliated $\Gamma$-manifolds.

The fourth chapter is the main part of the work and it is divided in two parts. In the first one, given a foliated $\Gamma$-manifold $(M, \mathcal{F}, \Gamma)$, with $\Gamma$ a compact Lie group, and given the groupoid $H(M, \mathcal{F}, \Gamma)$ we define a Haefliger-type transverse (co-)homology on $(M, \mathcal{F})$ with respect to the (equivalence class of ) pseudogroup $P_{\Gamma}$ determined by $H(M, \mathcal{F}, \Gamma)$. Then, using closed $P_{\Gamma}$-invariant currents we can define continuous equivariant cyclic cocycles $\phi_{C}$ on $\left(C_{c}^{\infty}(H(M, \mathcal{F}, \Gamma)), \Gamma\right)$. An important example is provided by the equivariant cyclic cocycle corresponding to the transverse orientation of a transversely oriented foliated $\Gamma$-manifold. As already mentioned, our construction is formally analogous to that in [7], but since we use the groupoid $H(M, \mathcal{F}, \Gamma)$ instead of the holonomy groupoid $H(\mathcal{F})$, we can deal with general $\Gamma$-actions by leaf-preserving diffeomorphisms and not only by holonomy diffeomorphisms (see [7]). Now, using even dimensional equivariant cyclic cocycles, we will be able to define higher Lefschetz numbers $L_{\phi_{C}}([h], D)$ of the conjugacy class $[h]$ of an element $h \in \Gamma$ with respect to an elliptic $\Gamma$-equivariant pseudodifferential operator $D$.

In the second part, we focus on a remarkable equivariant cyclic cocycle, namely the equivariant Godbillon-Vey cyclic cocycle. We first review the construction of the (nonequivariant) Godbillon-Vey cyclic cocycle for codimension 1 foliated bundles as given in [41]. Then, we generalize the construction of [41] to an arbitrary transversely oriented foliated $\Gamma$-manifold of codimension 1 in order to define a cyclic 2 -cocycle gv on $C_{c}^{\infty}(H(M, \mathcal{F}, \Gamma))$. Finally, we construct an equivariant cyclic cocycle $\mathbf{g v}^{\Gamma}$, whose evaluation at the identity of $\Gamma$ coincides with the Godbillon-Vey cyclic cocycle $\mathbf{g v}$. Using $\mathbf{g v}^{\Gamma}$ we can define the Godbillon-Vey Lefschetz number $L_{\mathbf{g v}}([h], D)$ of the conjugacy class $[h]$ of the element $h \in \Gamma$ with respect to the $\Gamma$-invariant elliptic pseudodifferential operator $D$.

At this point it is natural to ask how to compute the defined higher Lefschetz numbers. However, this question will not be addressed here for the following reasons. First, our equivariant cyclic cocycles are defined on the algebra $C_{c}^{\infty}(H(M, \mathcal{F}, \Gamma))$. However, in order to compute the higher Lefschetz numbers, we should extend the equivariant cyclic cocycles to holomorphically closed subalgebras containing $C_{c}^{\infty}(H(M, \mathcal{F}, \Gamma))$. This extension process is, however, a delicate point of the theory (see [17, Chapter III for further comments). Secondly, we cannot apply the equivariant Benameur-Connes-Skandalis longitudinal index theorem [5], since this is formulated in terms of the holonomy groupoid $H(\mathcal{F})$ and not in terms of $H(M, \mathcal{F}, \Gamma)$. Therefore, one needs first a suitable generalization of this theorem to encompass these more general groupoids. Alternatively, to obtain equivariant index formulas one could maybe use the approach through superconnections developed in [26]. This method has to be generalized to the 
equivariant case as well. These developments will be treated elsewhere. 


\section{Chapter 1}

\section{Foliations}

\subsection{Basic definitions and some examples}

Foliated manifolds are nowadays an important and still developing research topic in Mathematics. The origin of this notion can be traced back to the works of Ehresmann, Reeb, Haefliger and many others in the '50, who started sistematically to investigate these objects.

At the present foliated manifolds can be studied with a variety of powerful techniques, developed over the years and coming from different areas of the Mathematics. There is a homotopy theoretic approach that culminates in some beautiful results by Thurston, about quantitative aspects of the theory of foliations, or by Tsuboi. The interested reader is referred to 32 and 44 and the references therein. At the other side one can think of a foliation as a generalized dynamical system and then study dynamical and ergodic properties of a foliated manifold.

A further and powerful approach to the study of a foliated manifold comes from looking at it as a so called " noncommutative space". We refer to [?] for a more conceptual explanation of this name and for a brief historical 'excursus'. Here we just would like to emphasize that the foliations provide one of the basic class of examples of noncommutative spaces, that can be of course studied by methods of Noncommutative Geometry. Understanding these mathematical objects has maybe been one of the motivations and starting points in developing Noncommutative Geometry.

In this work we will adopt this point of view. Let us therefore begin by making more precise how a foliation gives rise to a noncommutative space and by introducing some basic invariants associated to it.

In the next sections and chapters we will go further using this approach, introducing other invariants for our foliations.

Let $M$ a manifold of class $C^{\infty}$ and dimension $n$. We consider open rectangular sets of $\mathbb{R}^{n}$, i.e. open sets $B \subseteq \mathbb{R}^{n}$ of the form $B=J_{1} \times J_{2} \times \ldots \times J_{n}$, where $J_{k}=\left(\alpha_{k}, \beta_{k}\right)$ is a connected open interval of the $k$-th coordinate axis of $\mathbb{R}^{n}$ possibly unbounded. 
Definition 1.1. A foliated chart on $M$ of codimension $q$ and class $C^{l}$ is a pair $(U, \phi)$ where $U \subseteq M$ is an open set and

$$
\phi: U \rightarrow B^{n-q} \times B^{q} \subseteq \mathbb{R}^{n-q} \times \mathbb{R}^{q}
$$

is a diffeomorphism of class $C^{l}$. Here $B^{n-q}$ and $B^{q}$ are open rectangular sets, respectively, of $\mathbb{R}^{n-q}$ and $\mathbb{R}^{q}$

Define the plaques of the foliated chart $(U, \phi)$ as the level sets

$$
P_{y}=\phi^{-1}\left(B^{n-q} \times\{y\}\right) \subset U
$$

for all $y \in B^{q}$. Similarly we define the transversals as

$$
T_{x}=\phi^{-1}\left(\{x\} \times B^{q}\right) \subset U
$$

for all $x \in B^{n-q}$ Troughout we will often denote a foliated chart $(U, x, y)$ instead of $(U, \phi)$, showing explicitly the longitudinal and transverse coordinates.

Definition 1.2. 1. A regular foliated atlas of codimension $q$ and of class $C^{l}$ on the smooth manifold $M$ is an atlas $\mathcal{U}=\left\{\left(U_{i}, \phi_{i}\right)\right\}_{i \in \mathcal{L}}$ of class $C^{l}$ on $M$ made up by foliated charts of codimension $q$ such that $\forall i, j \in \mathcal{L}$ for which $U_{i} \cap U_{j} \neq \emptyset$ the coordinate transformations

$$
\gamma_{i j}:=\phi_{i} \circ \phi_{j}^{-1}: \phi_{j}\left(U_{i} \cap U_{j}\right) \rightarrow \phi_{i}\left(U_{i} \cap U_{j}\right)
$$

have the form

$$
\gamma_{i j}\left(x_{j}, y_{j}\right)=\left(x_{i}\left(x_{j}, y_{j}\right), y_{i}\left(y_{j}\right)\right)
$$

2. We will say that two regular foliated atlases $\mathcal{U}=\left\{\left(U_{i}, \phi_{i}\right)\right\}$ and $\mathcal{V}=$ $\left\{\left(V_{j}, \psi_{j}\right)\right\}$ are coherent if their union is again a regular foliated atlas.

In other words in the definition of regular foliated atlas we demand that, in the case $U_{i} \cap U_{j} \neq \emptyset$, each plaque $P \subset U_{i}$ intersects at most one plaque $Q \subset U_{j}$ and that $P \cap Q$ is open both in $P$ and $Q$. In this way we prevent the possibility that a plaque $P$ might intersect distinct plaques of another foliated chart. (see, for instance [1] for further remarks.)

It is not difficult to prove that coherence of regular foliated atlases is an equivalence relation.

Definition 1.3. A foliation $\mathcal{F}$ on $M$ of codimension $q$ and of class $C^{l}$ is a coherence class of regular foliated atlases on $M$ of codimension $q$ and class $C^{l}$

In the following we will assume the foliated atlas to be of class $C^{\infty}$. By Zorn's lemma (cf. [2], pagg. 3-4) every coherence class of regular foliated atlases contains a unique maximal regular foliated atlas. Therefore we can equivalently define a foliation $\mathcal{F}$ of codimension $q$ on a smooth manifold $M$ to be a maximal regular foliated atlas of codimension $q$. 
Remark 1.1. Notice that the transverse coordinate map $y_{i}$ can be thought of as a submersion

$$
y_{i}: U_{i} \rightarrow B^{q} \subset \mathbb{R}^{q}
$$

and that the transverse coordinate transformations a regular foliated atlas $y_{i}\left(y_{j}\right)$ induces a local diffeomorphism of $R^{q}$ (whose regularity depends of course upon the regularity of the atlas)

$$
\gamma_{i j}: y_{j}\left(U_{i} \cap U_{j}\right) \subset R^{q} \rightarrow y_{i}\left(U_{i} \cap U_{j}\right) \subset R^{q}
$$

These local diffeomorphisms satisfy the cocycle conditions, i.e. on $y_{k}\left(U_{i} \cap U_{j} \cap\right.$ $\left.U_{k}\right)$

$$
\gamma_{i j} \circ \gamma_{j k}=\gamma_{i k}
$$

and moreover it holds that

$$
y_{i}=\gamma_{i j} \circ y_{j}
$$

The local diffeomorphisms $\gamma_{i j}$ play a crucial role in Foliation theory. They lead, indeed, to the notion of holonomy of the foliated manifold $(M, \mathcal{F})$. The set $\gamma=\left\{\gamma_{i j}\right\}$ is called the holonomy cocycle associated to the regular foliated atlas. It is nowadays well established that the set of germs of all possible holonomy transformations $\gamma_{i j}$ is a Lie groupoid and that this Lie groupoid models the transverse geometry of the foliation. We will deal with these ideas in the coming sections.

However for further remarks see [12, 11], 14] and the references therein.

Let $(M, \mathcal{F})$ be a foliated manifold, where $\operatorname{dim}(M)=n$ and $\operatorname{codim} \mathcal{F}=q$.

Lemma 1.1.1. There exists a family $\left\{L_{\lambda}, i_{\lambda}\right\}_{\lambda \in \Lambda}$ of dimension $n-q$ connected $C^{\infty}$-manifolds and $C^{\infty}-$ maps $i_{\lambda}: L_{\lambda} \rightarrow M$ such that

1. $i_{\lambda}: L_{\lambda} \rightarrow M$ are injective immersions.

2. $M=\bigsqcup_{\lambda \in \Lambda} L_{\lambda}$ (disjoint union)

3. If $(U, x, y) \in \mathcal{F}$ is a foliated chart and $L_{p}$ is the unique submanifold through a point $p \in U$, then $U \cap L_{p}$ is a union of plaques.

Proof. Let $\mathcal{F}=\left\{U_{j}, x_{j}, y_{j}\right\}$ denote the maximal regular foliated atlas defining the foliation on $M$. Let us introduce the following equivalence relation: for any two points $x, y \in M, x \sim y$ iff either there is a $\mathcal{F}$-plaque $P_{0}$ so that $x, y \in P_{0}$ or there exists a finite family of $\mathcal{F}$-plaques $\left\{P_{0}, P_{1}, \ldots, P_{m}\right\}$ such that

1. $x \in P_{0}$ and $y \in P_{m}$.

2. $P_{i} \cap P_{i+1} \neq \varnothing$, for all $i=0, \ldots, m-1$

The family of plaques $\left\{P_{0}, P_{1}, \ldots, P_{m}\right\}$ is called chain plaque of lenght $m$ connecting $x$ with $y$. It is obvious that this is indeed an equivalence relation. Denote by $L$ each equivalence class and by $\Lambda=M / \sim$ the set of equivalence classes.

The open subsets of the plaques $P \subseteq L$ generate a topology on $L$ which makes it 
into a Hausdorff, 2nd countable connected topological space. Moreover, since $\mathcal{F}$ is a regular foliated atlas, one knows that $\mathcal{F}$-plaques can only overlap in open subsets of each other. Therefore each equivalence class $L$ endowed with the above topology is a manifold of dimension $n-q$. If we denote now the inclusions by

$$
i_{\lambda}: L_{\lambda} \rightarrow M
$$

for each $\lambda \in \Lambda$, it is clear that these are injective, smooth and immersions with respect to the smooth structure just defined on $L_{\lambda}$.

It follows also immediately from the fact that $\sim$ is an equivalence relation that $M=\bigsqcup_{\lambda \in \Lambda} L_{\lambda}$, so 2) is proved. Finally, by definition of the equivalence classes themselves one has that $L \cap U$ is a union of plaques $P \subseteq U$, for any foliated chart $(U, x, y) \in \mathcal{F}$.

The submanifolds $i_{\lambda}\left(L_{\lambda}\right)$ of $(M, \mathcal{F})$, are called the leaves of the foliation. With a small abuse of notation we will identify the manifolds $L_{\lambda}$ with their images $i_{\lambda}\left(L_{\lambda}\right)$ through the immersions $i_{\lambda}$ and call them also the leaves of $(M, \mathcal{F})$.

Remark 1.2. An equivalent definition of foliation on a smooth manifold $M$ starts with a decomposition of $M$ in the disjoint union of a family of topologically immersed submanifolds $L_{\lambda}$ together with an atlas of foliated charts which is adapted to the decomposition. This means that for any foliated chart $(U, \phi)$ in the given atlas and any submanifold $L_{\lambda}$ the intersection $L_{\lambda} \cap U$, if not empty, is a union of the plaques of $(U, \phi)$.

Remark 1.3. In general a leaf $L$ in $(M, \mathcal{F})$ need not be an embedded submanifold of $M$, i.e. in general the immersion $i: L \rightarrow M$ is not an embedding. This is because the topology on each $L$, generated by the open subsets of each plaque $P \subseteq L$, may not coincide with the subspace topology of $L$ as subset of $M$. One simple example, showing this, is given by the Kronecker foliation $\left(T^{2}, \mathcal{F}_{\theta}\right)$ on the 2 -torus with slope $\theta \in \mathbb{R} \mathbb{Q}$.

Actually the proof of the lemma suggests that there is another topology on $M$ which is generated by all possible open subsets of any plaque in the maximal foliated atlas $\mathcal{F}$. With respect to this topology $M$ is a smooth manifold of dimension $\operatorname{dim} \mathcal{F}$, disconnected with connected components exactly the leaves $L$. This topology is often referred to as the leaf topology.

A further important feature appearing in the proof of lemma 1.1.1 is that a foliation naturally defines an equivalence relation. Set $\mathcal{R}_{\mathcal{F}}=\left\{\left(p, p^{\prime}\right) \in M \times M\right.$ : $\left.p \sim p^{\prime}\right\}$.

Definition 1.4. We will call $\mathcal{R}_{\mathcal{F}}$ the equivalence relation of the foliation $(M, \mathcal{F})$

The graph of this equivalence relation is a groupoid (see section 1.3 naturally associated to the foliation.

Let $(M, \mathcal{F})$ be a foliated manifold. For any $x \in M$, consider

$$
F_{x}:=T_{x} L_{x} \subset T_{x} M
$$


The collection

$$
F=\bigcup_{x \in M} F_{x}
$$

is a vector subbundle of $T M$, since the map $x \mapsto \operatorname{dim}\left(F_{x}\right)$ is a locally constant function on each connected component of $M$.

Definition 1.5. The smooth distribution $F \subset T M$ is called the vector bundle tangent to the foliation $\mathcal{F}$.

Moreover we have the natural short exact sequence of vector bundles on $M$

$$
0 \rightarrow F \rightarrow T M \rightarrow T M / F \rightarrow 0
$$

It is not difficult to check that $T M / F$ is a vector bundle of rank $q$.

Definition 1.6. The vector bundle $T M / F$ is called the normal bundle or the transverse bundle to the foliation $\mathcal{F}$

These two vector bundles naturally attached to a foliation $(M, \mathcal{F})$ play a crucial role in the theory of foliations. The transverse bundle is, for instance, fundamental for the construction of characteristic classes of a foliation, both geometrically and analytically. We will deal with some aspects of these considerations in the next chapters of this work.

In particular, let us give the following definition.

Definition 1.7. A foliated manifold $(M, \mathcal{F})$ is said to be transversely orientable if the normal bundle to the foliation $T M / F$ is orientable. The foliation is transversely oriented if it is transversely orientable and an orientation of $T M / F$ is chosen.

Transversely orientable foliated manifolds will play an important role in this work.

Now we will explain in which sense the tangent bundle $F$ to the foliation $(M, \mathcal{F})$ determines the foliation.

In particular we put the following problem: given a $C^{\infty}$-manifold $M$ of dimension $n$ and a distribution $F \subseteq T M$ of the tangent bundle of rank $p(p \leq n)$, does there exist a $C^{\infty}$ foliation $\mathcal{F}$ on $M$ of codimension $n-p$ such that $T(\mathcal{F})=F$ ? The solution to this question is provided by the classical theorem of Frobenius, that we would like briefly to mention.

Let us introduce the following notions

Definition 1.8. Let $F \subseteq T M$ be a $C^{\infty}$ distribution.

1. $F$ is said to be involutive if the smooth sections $C^{\infty}(F) \subseteq C^{\infty}(T M)$ is a Lie subalgebra of the Lie algebra of all smooth vector fields on $M$.

2. $F$ is completely integrable if $\forall x \in M$ there is a topologically immersed submanifold $N \subseteq M$ so that $x \in N$ and $T_{z} N=F_{z}, \forall z \in N$

3. A graded ideal $I^{*} \subseteq \Omega^{*}(M)$ is said to be differential if $d\left(I^{*}\right) \subseteq I^{*}$. 
Theorem 1.1.2. (Frobenius) Let $F$ be a rank $=p$ distribution of $T M$. The following are equivalent.

1. F is completely integrable.

2. The graded ideal $I^{*}(F) \subseteq \Omega^{*}(M)$ of smooth differential forms which vanish on $F$ is a differential graded ideal.

3. $F$ is involutive.

4. There exists a regular foliated atlas on $M$ of codimension $q=n-p$, such that every plaque in the atlas is an integral submanifold to $F$

As we stated the theorem, it is only a local result. However we have by definition the following global consequence

Corollary 1.1.3. If the rank $=p$ distribution $F \subseteq T M$ is completely integrable, then there is a $C^{\infty}$ foliation $\mathcal{F}$ of codimension $q=n-p$ on $M$ so that $T(\mathcal{F})=F$.

By virtue of the above theorem we get a negative answer to the integrability problem, we have put above. In fact, to have a foliation $(M, \mathcal{F})$ integrating a given distribution $F \subseteq T M$, one needs the smooth sections $C^{\infty}(F)$ tangent to $F$ to be closed with respect to Lie bracket of vector fields on $M$. There are indeed examples of not involutive distributions and not completely integrable distributions, even for $\mathbb{R}^{n}$, showing that the condition of the theorem of Frobenius is not trivial. For further remarks and proofs see [11] and the references therein. A further very important feature of the theorem above is that a foliation is completely determined by infinitesimal data, i.e. by the tangent bundle to the foliation. One might define therefore a foliated manifold to be a pair consisting of a smooth manifold $M$ with an involutive distribution $F \subseteq T M$.

Example 1.1. Let $\pi: V \rightarrow B$ a differentiable fiber bundle of class $C^{\infty}$ with tipical fibre the $p$-dimensional connected manifold $F$. Then the fibers $\pi^{-1}(b)$, for any $b \in B$, are the leaves of a natural foliation $\mathcal{F}$ of codimension $q=\operatorname{dim} B$ on the total space $V$. To see this, consider the distribution $T_{\text {vert }} V \subseteq T V$ defined by the vertical tangent vectors to the fibers

$$
T_{\text {vert }} V=\{v \in T V \mid d \pi(v)=0 .\}=\operatorname{ker}(d \pi)
$$

Since the pushforward $\pi_{*}$ is compatible with the Lie bracket on vector fields $\Gamma(T V)$, one immediately deduces that $\Gamma\left(T_{\text {vert }} V\right)$ is closed under the Lie bracket, i.e. $T_{\text {vert }} V$ is an involutive distribution. By Frobenius's theorem it defines a foliation $\mathcal{F}$ on $V$ of codimension $\operatorname{dim} V-\operatorname{rank} T_{\text {vert }} V$ such that $T \mathcal{F}=T_{\text {vert }} V$. It is now obvious that the leaves of this foliation are exactly the fibers $\pi^{-1}(b)$ for all $b \in B$.

The foliations arising in this way are called simple foliations. 
Example 1.2. Let $G$ be a Lie group and $H$ a connected Lie subgroup (not necessarily a closed subgroup). Thus one has that the left cosets $\{g H\}_{g \in G}$ are the leaves of a foliation on $G$. Indeed it is not difficult to check that the tangent vectors to the left cosets forms a distribution $G \times \mathfrak{h} \subseteq T G \cong G \times \mathfrak{g}$, where $\mathfrak{g}$ is the Lie algebra of $G$ and $\mathfrak{h}$ is the Lie subalgebra of the Lie subgroup $H$. It is now obvious that the distribution $G \times \mathfrak{h}$ is involutive and that the leaves of the foliation defined by it are the left cosets.

If $H$ is closed, then $G / H$ is a manifold and the canonical projection $\pi: G \rightarrow$ $G / H$ is a fibre bundle, whose fibers are again the left cosets by $H$.

In the above classes of examples all the leaves of the foliation are diffeomorphic to each other. This is false in general. We will construct in the next examples classes of foliations for which the leaves need not be all diffeomorphic to each other.

Example 1.3. (foliated bundle). Let $H$ be a finitely generated discrete group and $\tilde{M}$ be a smooth manifold on which $H$ acts properly and freely on the right. The quotient space $M=\tilde{M} / H$ is thus a smooth manifold. Let $T$ be a smooth connected manifold and assume $H$ acts on it on the right by diffeomorphisms. Consider the product manifold $\tilde{M} \times T$ endowed with the diagonal action of $H$ on the right. Thus we have the $H$-equivariant fibre bundle

$$
\pi: \tilde{M} \times T \rightarrow T
$$

Since the action of $H$ on $\tilde{M}$ is proper and free, the diagonal action on $\tilde{M} \times T$ is also proper and free. We denote the quotient manifold by $X=(\tilde{M} \times T) / H$.

Proposition 1.1.4. 1. The manifold $X$ is the total space of the $C^{\infty}$ fibre bundle

$$
p: X \rightarrow M
$$

over the manifold $M=\tilde{M} / H$ with typical fibre the manifold $T$.

2. Moreover $X$ inherits a $C^{\infty}$ foliation $\mathcal{F}$ of codimension $q=\operatorname{dim}(T)$, whose leaves are the images of the fibres of $\pi: \tilde{M} \times T \rightarrow T$ under the quotient map $\tilde{M} \times T \rightarrow X$.

3. For any point $m \in M$, the fibre $p^{-1}(m)$ is a complete transversal submanifold to the foliation $\mathcal{F}$ and the bundle projection $p$ restricted to any leaf $L$ of $\mathcal{F}$ is a covering map.

For a proof of this proposition and further comments, we refer to [1] and the references therein.

Definition 1.9. The triple $(X, p, \mathcal{F})$ is called a $T$-foliated bundle or, simply, a foliated bundle.

Foliated bundles constitutes an important class of foliated manifolds, that we will study in some detail in following sections and chapters of this work. 
Example 1.4. (group actions) Let $G$ be a connected Lie group acting smoothly on a connected $C^{\infty}$ manifold $M$. Assume that the dimension of the isotropy groups $G_{x}=\{g \in G: g(x)=x\}$ is indipendent of $x \in M$. In particular one could consider locally free smooth actions of connected Lie groups, meaning that the isotropy groups have to be discrete.

Then the orbits $G x$ of the action are immersed submanifolds of $M$ of constant dimension $p=\operatorname{dim} G-\operatorname{dim} G_{x}$ for all points $x \in M$ and define a foliation on $M$.

Roughly speaking, many information, both geometrical-topological and dynamical, is encoded in the transverse structure of the foliation and in the so called "space of leaves" of the foliation. We end this section with the following

Definition 1.10. Let $(M, \mathcal{F})$ a foliated manifold. The set of leaves $M / \mathcal{F}$ is the set of leaves of $\mathcal{F}$, i.e.

$$
M / \mathcal{F}=\{L \subseteq M: L \in \mathcal{F}\}
$$

One has the natural projection

$$
p: M \rightarrow M / \mathcal{F}
$$

which sends $x \in M$ to the (unique) leaf $L_{x}$ passing through $x$.

We may endowe the set of leaves with the quotient topology with respect to $p$. However with this topology the space of leaves is very often a very ill-behaved and singular space.

To deal with it we will think of it as a noncommutative space.

\subsection{Groups of diffeomorphisms preserving the foliation}

In the last sections we gave the basic definitions and examples of a foliated manifold $(M, \mathcal{F})$.

In this section, given a Lie group $\Gamma$ we will define the most basic object of study of the present work, the so-called foliated $\Gamma$-manifold. These are simply foliated manifolds endowed with a Lie group action of diffeomorphisms preserving the foliation. Thus, our primary task is to introduce these groups of foliation-preserving diffeomorphisms. It turns out that these groups encode in a complicated way important informations about the foliation, especially regarding its transverse structure. In next sections we will see how some of these informations can be extracted. In the present one we will just study some basic properties of them and show some examples of foliated $\Gamma$-manifolds.

Let $(M, \mathcal{F}))$ and $(N, \mathcal{G})$ denote foliated manifolds and $M / \mathcal{F}$ and $N / \mathcal{G}$, respectively, their space of leaves, as defined in the previous section.

Definition 1.11. 1 . A leafwise map $f:(M, \mathcal{F}) \rightarrow(N, \mathcal{G})$ is a smooth map $f: M \rightarrow N$ such that

$$
f(L) \subseteq L^{\prime}
$$


for any $L \in M / \mathcal{F}, L^{\prime} \in N / \mathcal{G}$.

2. A leafwise diffeomorphism $\phi:(M, \mathcal{F}) \rightarrow(N, \mathcal{G})$ is a diffeomorphism $\phi:$ $M \rightarrow N$ such that

$$
\phi(L)=L^{\prime}
$$

for all $L \in M / \mathcal{F}, L^{\prime} \in N / \mathcal{G}$

Denote $\operatorname{Diff}(M, \mathcal{F})$ the group of foliated diffeomorphisms of $(M, \mathcal{F})$ and $\operatorname{Diff}(\mathcal{F}) \unlhd \operatorname{Diff}(M, \mathcal{F})$ the normal subgroup of those foliated diffeomorphisms, which preserve each leaf of $(M, \mathcal{F})$, i.e. $f(L)=L$, for all $L \in M / \mathcal{F}$.

Lemma 1.2.1. Let $f: M \rightarrow N$ be a smooth map. The following are equivalent:

1. $f$ is a leafwise map.

2. $f$ preserves the equivalence relation groupoids associated to the foliations, i.e. $f\left(\mathcal{R}_{\mathcal{F}}\right) \subseteq \mathcal{R}_{\mathcal{G}}$.

3. the differential df $: T(M) \rightarrow T(N)$ sends $T \mathcal{F}$ to $T \mathcal{G}$.

Proof. 1. $\Longleftrightarrow 2$. If $f$ is a leafwise map between $(M, \mathcal{F})$ and $(N, \mathcal{G})$ then $f(x) \sim$ $f(y)$ in $N$, whenever $x \sim y$ in $M$. For the converse, observe simply that if $f$ preserves the equivalence relations, then it preserves also all equivalence classes. 1. $\Rightarrow 3$. Notice that for any leaf $L \in M / \mathcal{F}$, the restriction $f_{\mid L}: L \rightarrow L^{\prime}$ is a smooth map between leaves.

Let now $x \in M$ and $L_{x} \in M / \mathcal{F}$ be the leaf passing through $x$ and consider the restriction $f_{L_{x}}: L_{x} \rightarrow L_{f(x)}^{\prime}$. Since it is smooth, one has that $d\left(f_{L_{x}}\right)_{x}\left(T_{x}\left(L_{x}\right)\right) \subseteq$ $T_{f(x)}\left(L_{f(x)}^{\prime}\right)$. But one has that $T_{x} \mathcal{F}=T_{x}\left(L_{x}\right), T_{f(x)} \mathcal{G}=T_{f(x)}\left(L_{f(x)}^{\prime}\right)$ and $\left(d f_{x}\right)_{\mid F_{x}}=d\left(f_{L_{x}}\right)_{x}$. Thus one deduces that $d f_{x}\left(T_{x} \mathcal{F}\right) \subseteq T_{f(x)} \mathcal{G}$. Since $x$ is arbitrary we conclude that $d f(T \mathcal{F}) \subseteq T \mathcal{G}$.

3. $\Rightarrow 2$. Suppose that $x, y \in L$ and consider a leafwise path $\alpha:[0,1] \rightarrow L$ connecting $x$ with $y$. Then $f \cdot \alpha:[0,1] \rightarrow N$ is a path joining $f(x)$ with $f(y)$. Observe that $\forall t \in[0,1]$,

$$
\alpha^{\prime}(t) \in T_{\alpha(t)} \mathcal{F} .
$$

Choose a subdivision $\left\{0=t_{0}<t_{1}<\ldots<t_{m}=1\right\}$ of $[0,1]$ and foliated charts $\left\{U_{0}, U_{1}, \ldots, U_{m-1}\right\} \subseteq \mathcal{G}$ such that

$$
(f \cdot \alpha)\left(\left[t_{i}, t_{i+1}\right]\right) \subseteq U_{i} .
$$

Since $d f(T \mathcal{F}) \subseteq T \mathcal{G}$, one has

$$
d f_{f(\alpha(t))}\left(\alpha^{\prime}(t)\right) \in T_{f(\alpha(t))} \mathcal{G}
$$

and therefore there exists a plaque $P_{i} \subseteq U_{i}$ for all $i=0, \ldots, m-1$ such that

$$
(f \cdot \alpha)\left(\left[t_{i}, t_{i+1}\right]\right) \subseteq P_{i} .
$$

Thus there is a plaque chain $\left\{P_{0}, P_{1}, \ldots, P_{m-1}\right\}$ of lenght $m$ connecting $f(x)$ with $f(y)$. This implies that $f(x) \sim f(y)$, i.e. they are in the same leaf. 
Note that $\psi \in \operatorname{Diff}(\mathcal{F})$ if and only if $\operatorname{Graph}(\psi)=\{(x, \psi(x)) \mid x \in M\} \subseteq \mathcal{R}_{\mathcal{F}}$. The groups of diffeomorphisms defined above are in general very large as the following lemma states. In some sense the lemma is one of the main ingredients in the proof of the Frobenius theorem.

Recall that a vector field $X \in C^{\infty}(T M)$ on $M$ is called complete if it generates a one-parameter group of diffeomorphisms $\Phi=\left\{\phi_{t}\right\}$ for all $t \in \mathbb{R}$.

Lemma 1.2.2. 1. Let $(M, \mathcal{F})$ be a foliated manifold and $X \in C^{\infty}(T M)$ a complete smooth vector field. Let $\left\{\phi_{t}\right\}_{t \in \mathbb{R}}$ be the diffeomorphism flow generated by $X$. One has $\phi_{t} \in \operatorname{Diff}(M, \mathcal{F})$ for any $t \in \mathbb{R}$ if and only if $\left[X, C^{\infty}(T \mathcal{F})\right] \subseteq C^{\infty}(T \mathcal{F})$.

2. In particular $X \in C^{\infty}(T \mathcal{F})$ if and only if $\left\{\phi_{t}\right\}_{t \in \mathbb{R}} \subset \operatorname{Diff}(\mathcal{F})$.

Proof. 1. If, for all $t \in \mathbb{R} \phi_{t} \in \operatorname{Diff}(M, \mathcal{F})$, we already know by lemma 1.2 .1 that $T \mathcal{F}$ is also invariant under the flow. Thus, if $Y \in C^{\infty}(T \mathcal{F})$,

$$
[X, Y]=\lim _{t \rightarrow 0} \frac{\left(\phi_{-t}\right)_{*}(Y)-Y}{t} \in C^{\infty}(T \mathcal{F}) .
$$

Conversely, let us first assume that $t \in(-\epsilon, \epsilon)$, for $\epsilon$ small enough. We will prove that for such $t$ the diffeomorphism $\phi\{t$ carries plaques to plaques. The fact that $\phi_{t}$ carries plaques to plaques for all $t \in \mathbb{R}$ will then follow easily.

Let $(U, x, y) \in \mathcal{F}$. One has that

$$
X_{\mid U}=\sum_{i=1}^{p} f_{i}(x, y) \frac{\partial}{\partial x_{i}}+\sum_{j=1}^{q} t_{j}(x, y) \frac{\partial}{\partial y_{j}} .
$$

By hypothesis,

$$
\left[\frac{\partial}{\partial x_{i}}, X_{\mid U}\right] \in C^{\infty}(T \mathcal{F})
$$

therefore the functions $t_{j}(x, y)=t_{j}(y)$, i.e. they are indipendent of $x$, for all $j=1, \ldots, q$. The flow $\phi_{t}=\left(\phi_{t}^{1}(x, y, t), \phi_{t}^{2}(x, y, t), \ldots, \phi_{t}^{p+q}(x, y, t)\right)$ is by definition the solution of the system of differential equation

$$
\begin{array}{r}
\frac{d}{d t} \phi_{t}^{l}=f_{l}(x, y) \quad l=1, \ldots, p \\
\frac{d}{d t} \phi_{t}^{k}=t_{k}(y) \quad k=p+1, \ldots, p+q
\end{array}
$$

Given the initial condition $(a, b) \in U$, we deduce that the last $q$ coordinates of $\phi_{t}$ only depend on $b$ and $t$. Thus the plaque $y=y(b, 0)=b$ is mapped onto the plaque $y=y(b, t)$.

2. If $X \in C^{\infty}(T \mathcal{F})$, then, applying the same argument, one obtains that the coordinates $t_{j} \equiv 0$. The last $q$ coordinates of $\phi_{t}$ are therefore even indipendent of $t$. The claim follows. 
By virtue of the above lemma, we may define a very important subgroup of $\operatorname{Dif} f(\mathcal{F})$. Notice that each compactly supported vector field is complete. Thus consider the exponential map

$$
\exp : C_{c}^{\infty}(M, T \mathcal{F}) \rightarrow \operatorname{Diff}(\mathcal{F})
$$

which sends $X$ to $\exp (X)$, and the image $\exp \left(C_{c}^{\infty}(M, T \mathcal{F})\right) \subseteq \operatorname{Diff}(\mathcal{F})$.

Definition 1.12. The subgroup $\exp \mathcal{F}$ is the subgroup of $\operatorname{Diff}(\mathcal{F})$ generated by $\exp \left(C_{c}^{\infty}(M, T \mathcal{F})\right)$.

Lemma 1.2.3. The subgroup $\exp \mathcal{F}$ is a normal subgroup of Dif $f(\mathcal{F})$.

Proof. Let $X \in C_{c}^{\infty}(M, T \mathcal{F})$ and $f \in \operatorname{Diff}(\mathcal{F})$. It is not difficult to check that

$$
f \exp (X) f^{-1}=\exp \left(f_{*}(X)\right) \in \exp \mathcal{F}
$$

from which the claim follows.

Remark 1.4. The discussion above suggests that the the group $\operatorname{Diff}(M, \mathcal{F})$ and $\operatorname{Diff}(\mathcal{F})$ are regular infinite dimensional Lie groups with Lie algebra $C_{c}^{\infty}(M, T \mathcal{F})$.

One has the sequence of normal subgroups

$$
\exp \mathcal{F} \unlhd \operatorname{Diff}(\mathcal{F}) \unlhd \operatorname{Diff}(M, \mathcal{F})
$$

Definition 1.13. 1 . Let $f_{0}, f_{1}:(M, \mathcal{F}) \rightarrow(N, \mathcal{G})$ two leafwise maps. A leafwise homotopy between $f_{0}$ and $f_{1}$ is a $C^{\infty, 0}$ map $H: M \times[0,1] \rightarrow N$ such that $H(x, \cdot):[0,1] \rightarrow N$ is a leafwise path with starting point $f_{0}(x)$ and end point $f_{1}(x)$ for any $x \in M$.

If there is a leafwise homotopy, then the leafwise maps $f_{0}, f_{1}$ are said to be leafwise homotopic.

2. We shall say that two leafwise maps are leafwise isotopic if there is a $C^{\infty}$ leafwise homotopy between them. In this case we will write $f_{0} \sim_{\mathcal{F}} f_{1}$.

A $C^{\infty, 0} \operatorname{map} H: M \times[0,1] \rightarrow N$ is a continuous map such that $H_{t}: M \rightarrow N$ is a $C^{\infty}$ map for all $t \in[0,1]$. By definition one clearly has that $H_{t}$ is a leafwise map for any $t \in[0,1]$.

In particular, if $\psi_{0}, \psi_{1} \in \operatorname{Diff}(M, \mathcal{F})$ are leafwise homotopic through the leafwise homotopy $H$, then clearly $H_{t} \in \operatorname{Diff}(M, \mathcal{F})$. Further, if $\psi_{0}=H_{0}$ is so that $\psi_{0}(L)=L^{\prime}$, then $H_{t}(L)=L^{\prime}$ for all $L \in M / \mathcal{F}, L^{\prime} \in N / \mathcal{G}, t \in[0,1]$.

Consider the normal subgroup $\operatorname{Dif} f_{0}(\mathcal{F}) \unlhd \operatorname{Diff}(\mathcal{F})$ of leaf-preserving diffeomorphisms $\psi \sim \sim_{\mathcal{F}} i d$. (leafwise isotopic). One has the following short exact sequence of groups

$$
1 \rightarrow \operatorname{Diff} f_{0}(\mathcal{F}) \rightarrow \operatorname{Diff}(\mathcal{F}) \rightarrow \operatorname{Diff}(\mathcal{F}) / \operatorname{Diff} f_{0}(\mathcal{F}) \rightarrow 1
$$


Definition 1.14. We will call $\operatorname{Diff}(\mathcal{F}) / \operatorname{Dif} f_{0}(\mathcal{F})$ the leafwise mapping class group of the foliation.

The leafwise mapping class group can be also characterized as the group $\pi_{0}(\operatorname{Dif} f(\mathcal{F}))$ of path connectedcomponents of $\operatorname{Diff}(\mathcal{F})$. It might be interesting to study more deeply $\pi_{0}(\operatorname{Diff}(\mathcal{F}))$.

Lemma 1.2.4. $\exp \mathcal{F} \unlhd D i f f_{0}(\mathcal{F})$

Proof. Let $X \in C_{c}^{\infty}(M, T \mathcal{F})$. Then there is a natural leafwise isotopy between the identity and the leafwise diffeomorphism $\exp (X)$ given by

$$
H(m, t)=\exp (t X)(m)
$$

for $m \in M, t \in[0,1]$. Therefore $\exp \mathcal{F} \leq D$ if $f_{0}(\mathcal{F})$. Since $\exp \mathcal{F}$ is a normal subgroup of Diff $(\mathcal{F})$, it is a fortiori normal in Dif $f_{0}(\mathcal{F})$.

Thus one has the sequence of groups

$$
\exp \mathcal{F} \unlhd \operatorname{Diff} f_{0}(\mathcal{F}) \unlhd \operatorname{Diff}(\mathcal{F}) \unlhd \operatorname{Diff}(M, \mathcal{F})
$$

each inclusion of which defines a normal subgroup.

We will deal with foliated manifold $(M, \mathcal{F})$ equipped with the action of a Lie group by foliation-preserving diffeomorphisms. More precisely, let $\Gamma$ be a Lie group.

Definition 1.15. An action of $\Gamma$ on the foliated manifold $(M, \mathcal{F})$ is a smooth action $\rho$ of $\Gamma$ on the manifold $M$ such that $\rho_{h} \in \operatorname{Diff}(M, \mathcal{F})$ for any $h \in \Gamma$.

The map $\rho: \Gamma \rightarrow \operatorname{Diff}(M, \mathcal{F})$ defines of course a group homomorphism. Since we are primarily interested in fixed point formulae, we will mostly deal with $\operatorname{Dif} f(\mathcal{F})$ and its subgroups. Let us then give the following

Definition 1.16. A foliated manifold $(M, \mathcal{F})$ is called a foliated $\Gamma$-manifold, if there is an action $\rho$ of $\Gamma$ taking values in $\operatorname{Diff}(\mathcal{F})$. We will denote a foliated $\Gamma$-manifold by $(M, \mathcal{F}, \Gamma)$.

Example 1.5. Let $(M, \mathcal{F})$ be a foliated manifold. The most natural example of Lie group action by leave-preserving diffeomorphisms on $(M, \mathcal{F})$ is the following. Pick any element $\varphi \in \operatorname{Diff}(\mathcal{F})$ and consider the group $\Gamma$ generated by $\varphi$. This group is either isomorphic to $\mathbb{Z}$ or is isomorphic to the finite group $\mathbb{Z}_{m}$, if $\varphi^{m}=i d$ for some $m$.

With the obvious action, the triple $(M, \mathcal{F}, \Gamma)$ is a foliated $\Gamma$-manifold..

Example 1.6. A further simple, but interesting example is given by considering vector fields tangent to the foliation. Any $X \in C_{c}^{\infty}(M, T \mathcal{F})$ is in particular complete. Therefore by Lemma 1.2.2, we know that the diffeomorphisms flow $\left(\phi_{t}^{X}\right)_{t \in \mathbb{R}}$, generated by $X$, is a subgroup of $\operatorname{Diff}(\mathcal{F})$. Thus, letting $\mathbb{R}$ act on $(M, \mathcal{F})$ through the flow $\left(\phi_{t}^{X}\right)_{t \in \mathbb{R}}$, we obtain a foliated $\mathbb{R}$-manifold $(M, \mathcal{F}, \mathbb{R})$. 
Example 1.7. Let $\pi: V \rightarrow B$ a differentiable fiber bundle with typical fibre a $p$-dimensional connected manifold $F$. Then, as we have seen in Example 1.1 . one has the foliation $(V, \mathcal{F})$ whose leaves are just the fibers of $\pi$. In this case, of course, the group $\operatorname{Diff}(\mathcal{F})$ of leaf-preserving diffeomorphisms of $V$ coincides with the group of bundle isomorphisms $\operatorname{Diff}(V, \pi)=\{f: V \rightarrow V \mid f \in$ $\operatorname{Diff}(V), f \circ \pi=\pi\}$.

Each subgroup $\Gamma \leq \operatorname{Diff}(V, \pi)$ gives rise to a foliated $\Gamma$-manifold. In particular, if $V$ is compact, $g$ is a Riemannian metric on $V$, and $f \in \operatorname{Diff}(V, \pi) \cap$ $I s o(V, g)$, one may consider the topologically cyclic subgroup $T$ generated by $f$ in $I s o(V, g)$. The group $T$ is, in general, a subgroup of $\operatorname{Diff}(M, \mathcal{F})$. However it has got a dense subgroup $\Gamma \leq T$ which acts on $(V, \mathcal{F})$ by leaf-preserving diffeomorphisms. Thus $(V, \mathcal{F}, \Gamma)$ is a foliated $\Gamma$-manifold, such that each element $f \in \Gamma$ is an isometry of the chosen metric $g$ and $\Gamma$ is relatively compact in $\operatorname{Diff}(M, \mathcal{F})$.

Example 1.8. Consider the setting explained in Example 1.4. Then it is obvious that $G$ acts on $(M, \mathcal{F})$ by leaf-preserving diffeomorphisms. Each subgroup $\Gamma \leq G$ defines, by restriction, a foliated $\Gamma$-manifold. In particular, taking $\Gamma=T$ the maximal compact subgroup of $G$, one gets a foliated $T$-manifold with compact acting group.

In next sections we will see further examples of foliated $\Gamma$-manifolds.

The diffeomorphisms groups introduced above are important. Indeed we will see in the next section that the transverse structure of the foliation can be in some sense constructed from the group $\exp \mathcal{F}$.

Moreover if the foliation $(M, \mathcal{F})$ is a foliated $\Gamma$-manifold, there are groupoids modelling the transverse structure and encoding also the action by $\Gamma$.

\subsection{The leaf space and the holonomy groupoid}

The leaf space of a foliation reflects in some sense the transver se geometry of the foliation itself. It is nowadays well known, for instance, that many topological and geometrical invariants of the foliation, in particular those coming from Index Theory, are encoded in the transverse structure of the foliation, modelled on the leaf space. This section is thus devoted to introduce this transverse structure for any foliated manifold.

As first attempt to describe the transverse geometry we use the leaf space of the foliation. However the problem is that, even in the simplest examples, the leaf space of a foliation is a very ill-behaved object. Thus to be able to extract the informations in it, a very deep and poweful idea is to describe the transverse geometry of the foliation modelling the leaf space with a groupoid (see, for instance, 22, 39] or 38).

In a following section we will associate operator algebras to the leaf space, using one of the groupoids introduced in this section. These algebras are in general highly noncommutative, showing the pathological behaviour of the leaf space. 
The construction, that is presented here, is heavily based on the paper [1. Let $(M, \mathcal{F})$ be a foliated manifold. Recall that we have defined the leaf space of the foliated manifold to be the set

$$
M / \mathcal{F}=\{L \subseteq M: L \in \mathcal{F}\}
$$

and we may define the projection

$$
p: M \rightarrow M / \mathcal{F}
$$

which associates the leaf $L_{x} \in M / \mathcal{F}$ through any point $x \in M$.

Thus we can endow the leaf space $M / \mathcal{F}$ with the quotient topology. The simplest examples show that the leaf space with this topology is so singular that it is in practice useless.

The simplest example is perhaps the Kronecker foliation $\mathcal{F}_{\theta}$ with irrational slope $\theta$ on the 2 -dimensional torus $T^{2}$. This is an example of a minimal foliation, i.e. each leaf is dense in the ambient manifold $T^{2}$. Therefore it follows by the definition of quotient topology that the only open sets of $T^{2} / \mathcal{F}_{\theta}$ are the empty set $\emptyset$ and the whole space. In other words the leaf space $T^{2} / \mathcal{F}_{\theta}$ of the Kronecker foliation $\left(T^{2}, \mathcal{F}_{\theta}\right)$ is a trivial topological space.

This reflections force to look for a better model for the leaf space of a foliation. It turns out that a very powerful description of it can be achieved through the notion of groupoid.

Definition 1.17. A groupoid is a small category in which each arrow is invertible.

As it is customary, a groupoid is denoted by $G \rightrightarrows G^{(0)}$, where $G^{(0)}$ is the set of objects and $G$ is the set of arrows. The maps connecting $G$ with $G^{(0)}$ in the notation above are the source map $s: G \rightarrow G^{(0)}$ and the target map $t: G \rightarrow G^{(0)}$, defined for any $\gamma: x \rightarrow y$ in $G$ by

$$
s(\gamma)=x
$$

and

$$
t(\gamma)=y
$$

Since we are dealing with a category, we have a composition map

$$
m: G^{(2)}=\left\{\left(\gamma, \gamma^{\prime}\right) \in G \times G \mid s(\gamma)=t\left(\gamma^{\prime}\right)\right\} \rightarrow G
$$

given by the composition $m\left(\gamma, \gamma^{\prime}\right)=\gamma \gamma^{\prime}$.

Moreover since each arrow is by definition invertible, one also has the bijection $i: G \rightarrow G$ defined by

$$
i(\gamma)=\gamma^{-1}
$$

and the so-called inclusion of the units $u: G^{(0)} \hookrightarrow G$ defined by

$$
u(x)=1_{x}
$$

where $1_{x}: x \rightarrow x$ is the identity arrow. 
Definition 1.18. 1. A Lie groupoid is a groupoid $G \rightrightarrows G^{(0)}$ such that $G$ and $G^{(0)}$ are smooth manifolds, all the structure maps defined above are smooth, and $s, t: G \rightarrow G^{(0)}$ are submersions.

2. A Lie groupoid $G \rightrightarrows G^{(0)}$ is called étale if the source map $s: G \rightarrow G^{(0)}$ is a local diffeomorphism.

Remark 1.5. If the source map is a local diffeomorphism, then it follows that all other structure maps are also local diffeomorphisms.

Basic examples of groupoids are groups, equivalence relations and group actions on sets.

Examples of Lie groupoids are Lie groups, Lie group actions on manifolds, the Haefliger groupoid $\Gamma^{n}$ asociated with the pseudogroup of local diffeomorphisms of $\mathbb{R}^{n}$. For further examples, details and properties of Lie groupoids we refer to [17, 11], 8] and 39.

In the rest of this section and in the next ones we will construct various Lie groupoids naturally associated with a foliation $(M, \mathcal{F})$. As already mentioned at the beginning of the introduction we will use the deep methods developed in [1].

Definition 1.19. 1. Let $(M, \mathcal{F})$ be a foliated manifold. A bi-submersion of $(M, \mathcal{F})$ is a triple $(V, t, s)$ consisting of a smooth manifold $V$ together with smooth maps $t: V \rightarrow M$ and $s: V \rightarrow M$ satisfying the following

(a) $t$ and $s$ are submersions on their image.

(b) $t^{-1}(\mathcal{F})=s^{-1}(\mathcal{F})$, where these are the pullback foliations on $V$. We will sometimes denote $\mathcal{F}_{V}=s^{-1}(\mathcal{F})$.

(c) $C_{c}^{\infty}\left(V, T \mathcal{F}_{V}\right)=C_{c}^{\infty}(V$, kerds $)+C_{c}^{\infty}(V$, kerdt $)$

2. A bi-submersion $(V, t, s)$ is said to be leave-preserving if for any $v \in V$, $s(v)$ and $t(v)$ lie in the same leaf.

Roughly speaking, a bi-submersion is a kind of 'local Lie groupoid', which allows to study the foliation locally. We will mainly deal with leave-preserving bi-submersions.

Definition 1.20. Let $\left(V, t_{V}, s_{V}\right)$ and $\left(W, t_{W}, s_{W}\right)$ be bi-submersions of $(M, \mathcal{F})$. A morphism of bi-submersions $f:\left(V, t_{V}, s_{V}\right) \rightarrow\left(W, t_{W}, s_{W}\right)$ is a smooth map $f: V \rightarrow W$ such that $s_{V}(v)=s_{W}(f(v))$ and $t_{V}(v)=t_{W}(f(v))$.

Before introducing the bi-submersions that are needed for constructing the groupoids of a foliation, we observe some important features of bi-submersions. In the next proposition, we will see that each bi-submersion of $(M, \mathcal{F})$ has got an inverse and, moreover, any two bi-submersions of $(M, \mathcal{F})$ can be composed to give another bi-submersion of $(M, \mathcal{F})$.

Proposition 1.3.1. Let $(M, \mathcal{F})$ a foliated manifold and $\left(V, t_{V}, s_{V}\right)$ and $\left(W, t_{W}, s_{W}\right)$ be bi-submersions of $(M, \mathcal{F})$. 
1. The triple $\left(V, s_{V}, t_{V}\right)$ is a bi-submersion, which is called the inverse of $\left(V, t_{V}, s_{V}\right)$. We will denote the inverse by $V^{-1}$.

2. Set $Z=V_{s_{V}} \times_{t_{W}} W=\left\{(v, w) \in V \times W \mid s_{V}=t_{W}\right\}$ and $t_{Z}=t_{V} \circ p r_{1}$ and $s_{Z}=s_{W} \circ p r_{2}$. Then $\left(Z, t_{Z}, s_{Z}\right)$ is a bi-submersion, which is called the composition of $\left(V, t_{V}, s_{V}\right)$ and $\left(W, t_{W}, s_{W}\right)$. The composition will be denoted by $V \circ W$.

Proof. The proof of the first claim is obvious. For the second we refer to [1] where all the details are given.

The proposition above suggests to consider families of bi-submersions which are in some sense closed with respect to the operations above. More precisely

Definition 1.21. Let $\mathcal{V}=\left(V_{i}, t_{i}, s_{i}\right)_{i \in I}$ be a family of bi-submersions.

1. A bi-submersion $(U, t, s)$ is said to be adapted to $\mathcal{V}$ if for all $u \in U$ there is an open neighbourhood $W \subseteq U$ containing $u$, an index $i \in I$ and a morphism of bi-submersions $f: W \rightarrow V_{i}$.

2. The family $\mathcal{V}$ of bi-submersions is called an atlas if

(a) $\bigcup_{i \in I} s_{i}\left(V_{i}\right)=M$.

(b) The composition of any two elements of $\mathcal{V}$ is adapted to $\mathcal{V}$.

(c) The inverse of any element of $\mathcal{V}$ is adapted to $\mathcal{V}$.

3. Two atlases $\mathcal{U}$ and $\mathcal{V}$ are said to be equivalent if they are adapted to each other, i.e. if every element of $\mathcal{U}$ is adapted to $\mathcal{V}$ and conversely every element of $\mathcal{V}$ is adapted to $\mathcal{U}$

The notion of atlas is crucial in the description of the holonomy groupoid given in 1]. The point is that to any atlas one can associate a groupoid, i.e. loosely speaking the groupoid of germs of bisections of the elements of the atlas. In order to understand this let us give the following

Definition 1.22. Let $(V, t, s)$ be a bi-submersion.

1. A bisection of $(V, t, s)$ is a locally closed submanifold $T \subseteq V$ such that the restriction of both $t$ and $s$ is a diffeomorphism onto open subsets of $M$. The local diffeomorphism defined by a bisection is

$$
t_{\mid T} \circ s_{\mid T}^{-1}: s(T) \rightarrow t(T)
$$

2. A local diffeomorphism $\phi: \Omega \subseteq M \rightarrow M$ is carried by the bi-submersion $(V, t, s)$ at $v \in V$ if there is a bisection $T$ of $(V, t, s)$ containing $v$ whose local diffeomorphism coincide with $\phi$ in a neighborhood of $s(v)$. 
Remark 1.6. It follows directly from the definition that if a local diffeomorphism $\phi$ of $(M, \mathcal{F})$ is carried by a bi-submersion, then $\phi$ must preserve the foliation.

Moreover it is useful to observe that if two local diffeomorphisms $\phi$ and $\psi$ are carried respectively by $\left(U, t_{U}, s_{U}\right)$ at $u \in U$ and $\left(V, t_{V}, s_{V}\right)$ at $v \in V$ and $s_{U}(u)=t_{V}(v)$, then $\phi \circ \psi$ is carried by the composition $U \circ V$ at $(u, v)$.

Similarly the inverse of $\phi$ is carried by the inverse $U^{-1}$ at $u$.

Therefore one may think of a bisection as a 'geometric realization' of a local diffeomorphism preserving the foliation. Given any bi-submersion $(V, t, s)$ and any point $v \in V$, there exists a bisection $T$ containing $v$. For a proof of this see 1 .

Proposition 1.3.2. Let $(M, \mathcal{F})$ a foliated manifold and let $\operatorname{dim} M=n, \operatorname{dim} \mathcal{F}=$ $p$ and $\operatorname{codim} \mathcal{F}=n-p=q$. Moreover let $x \in M$.

1. (bi-submersions near the identities) Let $X_{1}, \ldots, X_{p} \in C_{c}^{\infty}(M, T \mathcal{F})$ be vector fields tangent to the foliation which are a local base frame for $T \mathcal{F}$ around $x$. For all $\lambda \in \mathbb{R}^{p}$ and all $y \in M$, set $\phi(\lambda, y)=\exp \left(\sum_{i} \lambda_{i} X_{i}\right)(y) \in \exp \mathcal{F}$. Define $\Omega_{0}=\mathbb{R}^{p} \times M$, the submersions $s_{0}(\lambda, y)=y$ and $t_{0}(\lambda, y)=\phi(\lambda, y)$. Then there exists an open neighborhood $\Omega \subseteq \Omega_{0}$ of $(0, x)$ such that $(\Omega, t=$ $\left.t_{0 \mid \Omega}, s=s_{0 \mid \Omega}\right)$ is a bi-submersion.

2. (minimality) If $(V, t, s)$ is another bi-submersion carrying the identity at $v \in V$ with $s(v)=x$, then there is an open neighborhood $V^{\prime} \subseteq V$ of $v$ and a local morphism of bi-submersions $\pi: V^{\prime} \rightarrow \Omega$ which is a submersion and $\pi(v)=(0, x)$.

Proof. We refer to 1 Proposition 2.10 for a detailed proof of the above result.

We will sometimes call identity bi-submersion, each of the bi-submersions constructed in 1.3 .21 ).

Corollary 1.3.3. 1. If $O \subseteq M$ is an open neighborhood of $x$ and $\phi: O \rightarrow$ $\Omega^{\prime}$ is a local diffeomorphism preserving the foliation, then there is a bisubmersion $(V, t, s)$ carrying $\phi$ at a point $v \in V$ such that $s(v)=x$.

2. If two bi-submersions $\left(U, t_{U}, s_{U}\right)$ and $\left(V, t_{V}, s_{V}\right)$ carry the same local diffeomorphism $\phi$ respectively at $u \in U$ and $v \in V$, then there exists an open neighborhhood $U^{\prime}$ of $u$ and a local morphism of bi-submersions $f: U^{\prime} \subseteq$ $U \rightarrow V$ such that $f(u)=v$.

3. If there is a local morphism of bi-submersions $h: V^{\prime} \subseteq V \rightarrow U$ with $h(v)=u$ for a point $v \in V^{\prime}$, then there is an open neighborhood $U^{\prime} \subseteq U$ of the point $u$ and a morphism of bi-submersions $f: U^{\prime} \rightarrow V$ so that $f(u)=v$. 
Proof. 1) Consider the point $x \in M$ and construct a bi-submersion $(\Omega, t, s)$ as constructed in 1.3 .21 ) around $x$. This bi-submersion carries the identity at $(0, x)$. Now up to reducing $\Omega$ we can suppose that $t(\Omega) \subseteq O$. Thus $(\Omega, \phi \circ t, s)$ is a bi-submersion carrying (a restriction of) $\phi$ at $(0, x)$.

2)As in 1) up to reducing $U$ and $V$ we have that $t_{U}(U)$ and $t_{V}(V)$ are contained in the range of $\phi$. Thus we can form new bi-submersions $\left(U, \phi^{-1} \circ t_{U}, s_{U}\right)$ and $\left(V, \phi^{-1} \circ t_{V}, s_{V}\right)$ which by construction carry the identity diffeomorphism respectively at $u \in U$ and $v \in V$. Now setting $s_{U}(u)=s_{V}(v)=x$, consider the identity bi-submersion $(\Omega, t, s)$ around $x$. We know from 1.3 .22$)$ that there exist open neighborhhoods $\tilde{U} \subseteq U$ of $u$ and $\tilde{V} \subseteq V$ of $v$ and morphisms of bi-submersions $h: \tilde{U} \rightarrow \Omega$ and $k: \tilde{V} \rightarrow \Omega$ with $h(u)=k(v)=(0, x)$ that are also submersions. Taking now a local section $s$ of $k$ such that $s(0, x)=v$, up to reducing $\tilde{U}$ we may assume the range of $h$ to be contained in the domain of $s$. Then we can set $f=s \circ h$.

3) Consider a bisection $T \subseteq V$ through $v \in V^{\prime}$ and let $\psi$ the local diffeomorphism associated to $T$. Since $h$ is a morphism of bi-submersions, $h(T)$ is a bi-section of $U$ through $h(v)=u$ and its associated diffeomorphism is $\psi$ again, because $h$ commutes with $s$ and $t$. Applying now 2), the claim follows.

Remark 1.7. It is important to note that in the identity bi-submersions constructed in 1.3 .21 ) the target submersion $t: \Omega \rightarrow M$ is defined by leavepreserving diffeomorphisms in the group $\exp \mathcal{F}$. (see section 1.2 ).

Remark 1.8. It is useful to note that the composition of identity bi-submersions can be described in a very simple form. Indeed, if $\left(\Omega_{1}, t_{1}, s_{1}\right)$ and $\left(\Omega_{2}, t_{2}, s_{2}\right)$ are two identity bi-submersions around, respectively, the points $x_{1}$ and $x_{2}$, their composition $\Omega_{2} \circ \Omega_{1}$ is by definition the set

$$
\left\{\left(\lambda^{2}, y^{2}\right),\left(\lambda^{1}, y^{1}\right) \in \Omega_{2} \times \Omega_{1} \mid \quad \exp \left(\sum \lambda_{i}^{1} X_{i}\right)\left(y^{1}\right)=y^{2}\right\} \subseteq \Omega_{2} \times \Omega_{1} .
$$

It is evident that the component $y^{2}$ is determined by the components $\lambda^{1}$ and $y^{1}$. Hence $\Omega_{2} \circ \Omega_{1}$ can be canonically identified with the open set

$$
\left\{\left(\lambda^{2}, \lambda^{1}, y^{1}\right) \mid \quad\left(\lambda^{2}, \exp \left(\sum \lambda_{i}^{1} X_{i}\right)\left(y^{1}\right) \in \Omega_{2},\left(\lambda^{1}, y^{1}\right) \in \Omega_{1}\right) \subseteq \mathbb{R}^{p} \times \mathbb{R}^{p} \times M\right.
$$

In what follows we will freely use both descriptions, wherever needed.

Let $\phi: \Omega \rightarrow \Omega^{\prime}$ be a local diffeomorphism preserving the foliation, $(V, t, s)$ be a bi-submersion and $x \in \Omega$. Suppose that $t(V)$ is contained in the domain of $\phi$. Then, as we did in the proof of corollary 1.3 .31 ), we may define a new bi-submersion $(V, \phi \circ t, s)$.

Definition 1.23. The bi-submersion $(V, \phi \circ t, s)$ is said to be twisted by the diffeomorphism $\phi$. 
Let $(M, \mathcal{F})$ be a foliated manifold and take a cover of $M$ by identity bisubmersions $\left\{\left(\Omega_{i}, t_{i}, s_{i}\right)\right\}_{i \in I}$ as given in 1.3 .21$)$. We consider the atlas $\mathcal{U}_{\tau}$ generated by those.

Moreover we consider also the maximal atlas $\mathcal{U}$ generated by all bi-submersions of this form.

Definition 1.24. As in [1] we will call the atlas $\mathcal{U}$ the path holonomy atlas and $\mathcal{U}_{\tau}$ the path holonomy atlas associated to the cover $\left\{\left(\Omega_{i}, t_{i}, s_{i}\right)\right\}$

The crucial theorem, valid for any atlas of bi-submersions, is the following

Theorem 1.3.4. Let $\mathcal{V}=\left(V_{i}, t_{i}, s_{i}\right)_{i \in I}$ be an atlas.

1. There is a natural equivalence relation on the manifold $\bigsqcup_{i \in I} V_{i}$ given by: $V_{i} \ni u \sim v \in V_{j}$ if and only if there is an open neighborhhood $V^{\prime} \subseteq V_{i}$ of $u$ and a morphism of bi-submersion $f: V^{\prime} \rightarrow V_{j}$ so that $f(u)=v$. We denote the set of equivalence classes by $G_{\mathcal{V}}$ and by $\pi=\left(\pi_{i}\right): \bigsqcup_{i \in I} V_{i} \rightarrow G_{\mathcal{V}}$ the quotient map.

2. There are two maps $s, t: G_{\mathcal{V}} \rightarrow M$, such that $s \circ \pi_{i}=s_{i}$ and $t \circ \pi_{i}=t_{i}$.

3. $G_{\mathcal{V}}$ is a groupoid with space of objects given by $M$ and source and target maps given respectively by $s$ and $t$. Moreover $\left(G_{\mathcal{V}}, t, s\right)$ is a topological groupoid if endowed with the quotient topology.

Proof. 1). The relation $\sim$ defined on $\bigsqcup_{i \in I} V_{i}$ is an equivalence realtion. Indeed, the reflexivity and transitivity are clear. The fact that it is symmetric follows from Corollary 1.3 .3 3).

2). Let us observe first that if $V_{i} \ni u \sim v \in V_{j}$, then $s_{i}(u)=s_{j}(v) \in M$ and $t_{i}(u)=t_{j}(v) \in M$. Thus we define the surjective map $s: G_{\mathcal{V}} \rightarrow M$ by

$$
G_{\mathcal{V}} \ni[u] \mapsto s_{i}(u) \in M
$$

if $u \in V_{i}$ is a representative of the element $[u] \in G_{\mathcal{V}}$. From the observation above we know that this map is well defined. Moreover it is also clear, from the definition, that for all $i \in I$ and $v \in V_{i}, s\left(\pi_{i}(v)\right)=s_{i}(v)$.

For the map $t: G_{\mathcal{V}} \rightarrow M$ the proof is the same.

$3)$. Let $[u],[v] \in G_{\mathcal{V}}$ such that $t([u])=s([v])$. This means that, if $u \in V_{i}$ and $v \in V_{j}$, one has

$$
t_{i}(u)=s_{j}(v)
$$

Consider the composition $V_{j} \circ V_{i}$ of bi-submersions and notice that $(v, u) \in V_{j} \circ V_{i}$. Since $V_{j} \circ V_{i}$ is adapted to the atlas, there is the quotient map (see [1] for further details)

$$
\pi_{V_{j} \circ V_{i}}: V_{j} \circ V_{i} \rightarrow G_{\mathcal{V}}
$$

We define the composition of the elements $[u]$ and $[v]$ by

$$
[v] \cdot[u]:=\pi_{V_{j} \circ V_{i}}(v, u) .
$$


This definition is well-posed. In fact, let us give $(W, t, s)$ and $\left(Z, t^{\prime}, s^{\prime}\right)$, bisubmersions adapted to $\mathcal{V}$, and $w \in W, z \in Z$ such that

$$
\pi_{W}(w)=\pi_{i}(u)=[u] \quad \pi_{Z}(z)=\pi_{j}(v)=[v] .
$$

A very important consequence of Corollary 1.3 .3 is that, for any two bi-submersions $(E, t, s)$ and $\left(F, t^{\prime}, s^{\prime}\right)$ adapted to the atlas $\mathcal{V}$ and points $e \in E$ and $f \in F$, one has $\pi_{E}(e)=\pi_{F}(f)$ if and only if there exists a local diffeomorphism carried both by $E$ at $e$ and by $F$ at $f$.

Therefore, there is a local diffeomorphisms $\psi$ carried both by $W$ at $w$ and by $V_{i}$ at $u$, and, analogously, there is a local diffeomorphism $\phi$ carried both by $Z$ at $z$ and by $V_{j}$ at $v$.

From 1.37 it follows now that these local diffeomorphisms can be locally composed and from Remark 1.6 one deduces that $Z \circ W$ and $V_{j} \circ V_{i}$ carry the same diffeomorphism respectively at $(z, w)$ and $(v, u)$, namely the composition $\psi \circ \phi$ defined on a suitable open set. Thus it follows from Corollary 1.3 .3 that

$$
\pi_{Z \circ W}(z, w)=\pi_{V_{j} \circ V_{i}}(v, u) .
$$

This proves that the composition is well defined. Now it is quite simple to check that $G_{\mathcal{V}}$ endowed with the maps $s, t$ and with the composition just defined is a groupoid.

Finally, if one induces the quotient topology on $G_{\mathcal{V}}$ through the quotient map $\pi: \bigsqcup_{i \in I} V_{i} \rightarrow G_{\mathcal{V}}$ it is clear that all the structural maps of the groupoid become continuous with respect to this topology. Then $\left(G_{\mathcal{V}}, t, s\right)$ is a topological groupoid.

Definition 1.25. Given a foliated manifold $(M, \mathcal{F})$, the holonomy groupoid $H(M, \mathcal{F})$ of $(M, \mathcal{F})$ is the groupoid $G_{\mathcal{U}}$ associated to the path holonomy atlas $\mathcal{U}$.

If there is no need to specify explicitly the manifold $M$ we will also denote the holonomy groupoid $H(\mathcal{F})$.

There is another description of the holonomy groupoid of a foliation, given by Winkelnkemper in 47]. Let us sketch it, as well.

Let $x, y \in M$ be two points lying in the same leaf $L$ and let $\alpha:[0,1] \rightarrow L$ a continuous path in the leaf connecting $x$ with $y$. Since the foliation $(M, \mathcal{F})$ is defined by a maximal regular foliated atlas, we can find foliated charts $\left\{\left(U_{0}, x_{0}, y_{0}\right),\left(U_{1}, x_{1}, y_{1}\right), \ldots,\left(U_{l}, x_{l}, y_{l}\right\}\right.$ such that

1. $x \in U_{0}$ and $y \in U_{l}$.

2. $U_{i} \cap U_{i+1} \neq \varnothing$ for any $i=0, \ldots l-1$.

3. there is a subdivision $\left\{0=t_{0}<t_{1}<\ldots<t_{l}=1\right\}$ of $[0,1]$ such that

$$
\alpha\left(\left[t_{i}, t_{i+1}\right]\right) \subseteq U_{i}
$$


4. each plaque $P \subseteq U_{i}$ intersects at most one plaque $Q \subseteq U_{i+1}$.

Since $\alpha$ is a leafwise path and by 3) above, it follows immediately that there is a plaque chain $\left\{P_{0}, P_{1}, \ldots, P_{l}\right\}$ connecting $x \in P_{0}$ and $y \in P_{l}$ and such that $\alpha\left(\left[t_{i}, t_{i+1}\right]\right) \subseteq P_{i} \subseteq U_{i}$.

Consider now local transversals $T_{x}$ at $x$ and $T_{y}$ at $y$. Denoting $\gamma_{i, i+1}$ the transverse coordinate maps between the foliated charts $U_{i}$ and $U_{i+1}$, as in Remark 1.1 . one sees that the composition

$$
h_{\alpha}:=\gamma_{l-1, l} \circ \gamma_{l-2, l-1} \circ \ldots \circ \gamma_{1,0}
$$

is a local diffeomorphism defined on a suitable open neighborhood of $x$ in $T_{x}$ onto an open neighborhood of $y$ in $T_{y}$ so that $h_{\alpha}(x)=y$. Such a local diffeomorphism is called a holonomy transformation.

A holonomy transformation depends on all the choices we have done for defining it. However the germ $\left(h_{\alpha}\right)_{x}$ of $h_{\alpha}$ at $x$ satisfies the following important feature.

Proposition 1.3.5. The germ $\left(h_{\alpha}\right)_{x}$ of the holonomy transformation $h_{\alpha}$ depends only on the homotopy class $[\alpha]$ of the path $\alpha$ in the leaf $L$ (rel $\partial \alpha)$.

For a proof of this result we refer to [1], chapter 2., pag. 60.

Consider, now, the space $C([0,1], \mathcal{F})$ of all continuous (with respect to the leaf topology) leafwise paths. This space has got a partially defined multiplication simply given by concatenation of paths.

Definition 1.26. [47] The holonomy groupoid $\operatorname{Graph}(M, \mathcal{F})$ of the foliated manifold $(M, \mathcal{F})$ is the quotient of $C([0,1], \mathcal{F})$ by the following equivalence relation. Two paths $\alpha$ and $\beta$ are equivalent if and only if they have the same initial point $\alpha(0)=\beta(0)=x$ and terminal point $\alpha(1)=\beta(1)=y$ and if their germinal holonomies at $x$ coincide, i.e. $\left(h_{\alpha}\right)_{x}=\left(h_{\beta}\right)_{x}$.

The set $\operatorname{Graph}(M, \mathcal{F})$ is a groupoid with space of objects $\operatorname{Graph}(M, \mathcal{F})^{(0)}=$ $M$. The source and range maps $s, t: \operatorname{Graph}(M, \mathcal{F}) \rightarrow M$ are defined, respectively, by $s([\alpha])=\alpha(0)$ and $t([\alpha])=\alpha(1)$. These are well defined. Moreover the partially defined multiplication on $C([0,1], \mathcal{F})$ descends to a partially defined multiplication on $\operatorname{Graph}(M, \mathcal{F})$. Thus $[\alpha],[\beta] \in \operatorname{Graph}(M, \mathcal{F})$ are composable if and only if $t([\alpha])=s([\beta])$ and, in this case, $[\beta] \cdot[\alpha]$ can be representated by the concatenation of the paths $\alpha$ and $\beta$.

Proposition 1.3.6. ( 47$)$ Let $(M, \mathcal{F})$ be a foliated manifold of dimension $\operatorname{dim} \mathcal{F}=p$ and codimension codim $\mathcal{F}=q$. The holonomy groupoid $\operatorname{Graph}(M, \mathcal{F})$ of $(M, \mathcal{F})$ is a not necessarily Hausdorff Lie groupoid of dimension 2 dim $\mathcal{F}+$ $\operatorname{codim} \mathcal{F}$.

We will prove this proposition in the next section in the more general case of twisted holonomy groupoids.

Given a foliated manifold $(M, \mathcal{F})$ we have now two a priori different "holonomy groupoids", namely $H(\mathcal{F})$ and $\operatorname{Graph}(M, \mathcal{F})$. The following theorem provides that there is a unique holonomy groupoid for the foliation $(M, \mathcal{F})$. 
Theorem 1.3.7. ([1]]) The holonomy groupoid $H(\mathcal{F})$ coincides with the groupoid $\operatorname{Graph}(M, \mathcal{F})$ defined by Winkelnkemper. In particular $H(\mathcal{F})$ is a Lie groupoid.

From now on we will denote the holonomy groupoid of $(M, \mathcal{F})$ by $H(M, \mathcal{F})$ or, simpler, $H(\mathcal{F})$ and $H$, if there is no confusion.

It is very important to have different descriptions for the holonomy groupoid. Even if it is technically more involved, the description through bi-submersions allows to attach a holonomy groupoid to any singular foliation. For details about this point see [1].

Besides it follows from the above theorem and remark 1.7 that the holonomy groupoid is built out of elements of the group $\exp \mathcal{F}$. In other words, the holonomy transformations, whose germs are the elements of the holonomy groupoid, are restrictions of diffeomorphisms of $\exp \mathcal{F}$ to foliated charts (and thus to local transversals). This has led us to construct twisted holonomy groupoids, i.e. groupoids that contain also germs of leave-preserving diffeomorphisms not necessarily belonging to the group $\exp \mathcal{F}$. For details of the construction, remarks and applications we refer to the next sections.

On the other side, the approach using paths shows other features and advantages. For instance, the relationship between the monodromy groupoid of the foliation and the holonomy groupoid is clear through this description (see 19, Proposition 1). In the following, we will freely use both descriptions, whenever it will be needed.

We end up this section with a simple example.

Example 1.9. Let $(M, \mathcal{F})$ be a simple foliation, as given in 1.1. Thus, the manifold $M$ is the total space of a locally trivial fibration with bundle projection denoted by $\pi: M \rightarrow B$ and typical fiber $F$. For simplicity, we assume $F$ to be connected. The leaves of $\mathcal{F}$ are, by definition, the fibers $\pi^{-1}(b)$, for all $b \in B$.

In this case, the holonomy groupoid is isomorphic to the equivalence relation $\mathcal{R}_{\mathcal{F}}$ of $(M, \mathcal{F})$ given by "lying in the same leaf" (see 1.4). The equivalence relation $\mathcal{R}_{\mathcal{F}}$ is given by the fibred product

$$
M \times_{B} M=\{(x, y) \in M \times M \mid \pi(x)=\pi(y)\}
$$

In the case of a simple foliation, this is a Lie groupoid with target and source maps, respectively, the first and second projection. Now, since the fiber bundle is locally trivial, there is no nontrivial holonomy, so that each element of the holonomy groupoid is completely determined by its source and target points, determining therefore a unique element of $\mathcal{R}_{\mathcal{F}}$.

More generally, if a foliated manifold $(M, \mathcal{F})$ is without holonomy, the holonomy groupoid is nothing else but the equivalence relation of the foliation.

\subsection{Holonomy groupoid of a foliated $\Gamma$-manifold}

Let $\Gamma$ be a (not necessarily compact nor connected) Lie group and $(M, \mathcal{F}, \Gamma)$ a foliated $\Gamma$-manifold. Take a cover of $M$ by identity bi-submersions $\left\{\left(\Omega_{i}, t_{i}, s_{i}\right)\right\}_{i \in I}$ 
as given in 1.3 .21 .

The idea is to twist (see definition 1.23 each bi-submersion $\left(\Omega_{i}, t_{i}, s_{i}\right)$ with the leave-preserving diffeomorphisms defined by the elements of $\Gamma$. More precisely we consider the family of bi-submersions

$$
\left\{\left(\Omega_{i}, g \circ t_{i}, s_{i}\right) \mid i \in I, g \in \Gamma\right\}
$$

Since each $g$ acts as leave-preserving diffeomorphisms, each element in the family 1.42 is a leave-preserving bi-submersion.

Notice also that the identity element $g=1 \in \Gamma$ does twist trivially the bisubmersions and therefore $\left\{\left(\Omega_{i}, t_{i}, s_{i}\right)\right\}_{i \in I} \subseteq\left\{\left(\Omega_{i}, g \circ t_{i}, s_{i}\right) \mid i \in I, g \in \Gamma\right\}$.

Let us consider now the atlas $\mathcal{U}_{\tau}^{\Gamma}$ generated by the family of bi-submersions given by 1.42 and $\mathcal{U}^{\Gamma}$ the associated maximal atlas.

We will refer to $\mathcal{U}^{\Gamma}$ as the path holonomy atlas twisted by $\Gamma$, or simply by the twisted path holonomy atlas.

Remark 1.9. It is important to note that the group $\langle\exp (\mathcal{F}), \Gamma>\subseteq \operatorname{Diff}(\mathcal{F})$, generated by $\exp (\mathcal{F})$ and $\Gamma$, enters the definition of the twisted path holonomy atlas. Indeed, it follows from the definition of composition of bi-submersions (cf. Proposition 1.3.1) that composing any two twisted bi-submersions $\left(\Omega_{1}, g \circ\right.$ $\left.t_{1}, s_{1}\right),\left(\Omega_{2}, g \circ t_{2}, s_{2}\right) \in \mathcal{U}^{\Gamma}$, one gets a bi-submersion whose source and target maps are some suitable element of $\langle\exp (\mathcal{F}), \Gamma\rangle$.

Lemma 1.4.1. 1. The path holonomy atlas $\mathcal{U} \subseteq \mathcal{U}^{\Gamma}$.

2. Let us denote by $H(\mathcal{F}, \Gamma) \rightrightarrows M$ the groupoid associated with the twisted holonomy atlas $\mathcal{U}^{\Gamma}$. This is a topological groupoid with source and target maps, respectively, denoted by $s_{\mathcal{F}}^{\Gamma}$ and $t_{\mathcal{F}}^{\Gamma}$

3. The holonomy groupoid $H(\mathcal{F})$ is a subgroupoid of $H(\mathcal{F}, \Gamma)$.

Proof. The first claim is an immediate consequence of the inclusion

$$
\left\{\left(\Omega_{i}, t_{i}, s_{i}\right)\right\}_{i \in I} \subseteq\left\{\left(\Omega_{i}, g \circ t_{i}, s_{i}\right) \mid i \in I, g \in \Gamma\right\} .
$$

The second follows immediately from theorem 1.3.4.

Let us now prove 3 ). By point 1) we know, in particular, that the path holonomy atlas is adapted to the twisted path holonomy atlas. Therefore one obtains a natural map $j: H(\mathcal{F}) \rightarrow H(\mathcal{F}, \Gamma)$, which sends each element $[u] \in H(\mathcal{F})$ to the corresponding element $[u]_{\Gamma} \in H(\mathcal{F}, \Gamma)$. This map is a morphism of groupoids, or, equivalently, a functor between $H(\mathcal{F})$ and $H(\mathcal{F}, \Gamma)$. Indeed, let $[u] \in H(\mathcal{F})$ be represented by the point $u$ in the bi-submersion $(U, t, s)$. Then, as consequence of 1$), j([u])=[u]_{\Gamma} \in H(\mathcal{F}, \Gamma)$ can be represented by the same point $u \in U$. Thus

$$
s_{\mathcal{F}}^{\Gamma}(j([u]))=s_{\mathcal{F}}^{\Gamma}\left([u]_{\Gamma}\right)=s(u)=s_{\mathcal{F}}([u]) .
$$

The same argument, applied to the target maps, yields that $t_{\mathcal{F}}^{\Gamma} \circ j=t_{\mathcal{F}}$. It is also immediate to verify that $j([v][u])=j([v]) j([u])$, applying the definition of composition given by the formula 1.39 .

Moreover, it is straightforward from the definition of groupoid associated with an atlas that this morphism is injective. 
Definition 1.27. The groupoid $H(\mathcal{F}, \Gamma)$ is called the holonomy groupoid twisted by $\Gamma$, or, simpler, the twisted holonomy groupoid, if there is no need to specify the group $\Gamma$.

Proposition 1.4.2. $\quad$ 1. The twisted holonomy groupoid $H(\mathcal{F}, \Gamma) \rightrightarrows M$ has the structure of a not necessarily Hausdorff $C^{\infty}$ manifold of dimension $2 \operatorname{dim} \mathcal{F}+\operatorname{codim} \mathcal{F}$ with respect to the quotient topology.

2. All the structural maps of $H(\mathcal{F}, \Gamma)$ are smooth. Moreover, the source and target maps $t_{\mathcal{F}}^{\Gamma}, s_{\mathcal{F}}^{\Gamma}: H(\mathcal{F}, \Gamma) \rightarrow M$ are smooth submersions, making $H(\mathcal{F}, \Gamma)$ into a Lie groupoid.

Proof. Let $g \in H(\mathcal{F}, \Gamma)$, with $s_{\mathcal{F}}^{\Gamma}(g)=x$ and $t_{\mathcal{F}}^{\Gamma}(g)=y$.

Then there is a bi-submersion $\left(W, t_{W}, s_{W}\right)$ in the twisted path holonomy atlas and a point $w \in W$ such that

$$
\pi_{W}(w)=g
$$

where $\pi_{W}: W \rightarrow G$ is the quotient map.

Recall that given any bi-submersion $(U, t, s)$ and a point $u \in U$, there exists always a bisection $S \subseteq U$ containing $u$ (cf. [1, Proposition 2.7). Then, let $T \subseteq W$ be a bisection through $w$ and let $\phi: s_{W}(T) \rightarrow t_{W}(T)$ the local diffeomorphism associated with $T$. Notice that $x \in s_{W}(T)$ and $y \in t_{W}(T)$.

Choose compactly supported vector fields $X_{1}, \ldots, X_{p}$ tangent to the foliation such that $\left(X_{1}\right)_{x}, \ldots,\left(X_{p}\right)_{x}$ form a basis of $T_{x} \mathcal{F}$. By Proposition 1.3.2, there exists a bi-submersion $\left(\Omega_{0}, t_{0}, s_{0}\right)$ satisfying the following properties

1. $\left(\Omega_{0}, t_{0}, s_{0}\right)$ is in the twisted path holonomy atlas.

2. The bi-submersion is minimal, i.e. $\operatorname{dim} \Omega_{0}=2 \operatorname{dim} \mathcal{F}+\operatorname{codim} \mathcal{F}$.

3. $s_{0}(\lambda, y)=y$ for all $(\lambda, y) \in \Omega_{0}$. In particular $s_{0}(0, x)=x=s_{W}(w)$.

4. $\left(\Omega_{0}, t_{0}, s_{0}\right)$ carries the identity at $(0, x)$.

Now, put $\Omega^{\prime}=\left\{p \in \Omega_{0} \mid t_{0}(p) \in s_{W}(T)\right\}, s^{\prime}=\left(s_{0}\right)_{\mid \Omega^{\prime}}$ and $t^{\prime}=\left(t_{0}\right)_{\mid \Omega^{\prime}}$ and twist this bi-submersion with the diffeomorphism $\phi$.

Thus, the twisted bi-submersion $\left(\Omega^{\prime}, \phi \cdot t^{\prime}, s^{\prime}\right)$ carries at $(0, x)$ the same diffeomorphism carried by $\left(W, t_{W}, s_{W}\right)$ at $w$. By Corollary 1.3.3 2), we deduce that there exists an open neighborhood $\Omega \subseteq \Omega^{\prime}$ of $(0, x)$ and a morphism of bisubmersions $f: \Omega \rightarrow W$ such that $f(0, x)=w$.

Setting $t=t_{\mid \Omega}^{\prime}$ and $s=s_{\mid \Omega}^{\prime}$, we have, therefore, obtained a bi-submersion $(\Omega, \phi \cdot t, s)$ adapted to the twisted path holonomy atlas.

Claim 1. There is an open neighborhood $B^{p} \times U \subseteq \Omega$ of $(0, x)$ such that, for all $y \in U$, the target map $(\phi \cdot t)_{\mid B^{p} \times\{y\}}: B^{p} \times\{y\} \rightarrow L_{y}$ is a diffeomorphism onto its image. Here $L_{y}$ denotes the leaf through $y$. 
Being $\phi \in \operatorname{Diff}(\mathcal{F})$, we only have to prove that

$$
t_{\mid B^{p} \times\{y\}}=\exp _{y}: B^{p} \times\{y\} \rightarrow L_{y}
$$

is a diffeomorphism onto its image for a suitable open ball $B^{p} \subseteq \mathbb{R}^{p}$ and open neighborhood $U$ of $x$.

Since the vector fields $X_{1}, \ldots, X_{p}$ form a basis at $x$, there exists an open coordinate neighborhood $O^{\prime} \subseteq M$ of $x$, such that $X_{1}, \ldots, X_{p}$ form a basis of $T \mathcal{F}$ at each point $y \in O^{\prime}$ and the Lie algebra of all smooth vector fields on $O^{\prime}$ tangent to the foliation is generated by $X_{1}, \ldots, X_{p}$ (see [1]). We assume that $x=(0,0, \ldots, 0)$ with respect to the coordinates $y_{i}$ on $O^{\prime}$.

By the proof of the Frobenius theorem 1.1.2, we may further assume that on $O^{\prime}$ the vector fields commute with each other, i.e. $\left[X_{i}, X_{j}\right]=0$. Thus, there is an open subset $O \subseteq O^{\prime}$ containing $x$ and an open set $B_{\epsilon}^{p}(0) \times B_{\epsilon^{\prime}}^{q}(0) \subseteq \mathbb{R}^{p} \times \mathbb{R}^{q}$ such that the map

$$
\left(\lambda_{1}, \ldots, \lambda_{p}, y_{p+1}, \ldots, y_{p+q}\right) \rightarrow \exp \left(\sum_{i} \lambda_{i} X_{i}\right)\left(0, \ldots, 0, y_{p+1}, \ldots, y_{p+q}\right)
$$

defines a diffeomorphism $\Psi: B_{\epsilon}^{p}(0) \times B_{\epsilon^{\prime}}^{q}(0) \simeq O$. Thus $O$ is a foliated chart. Now, there is an open disc $B^{p} \subseteq B_{\epsilon}^{p}(0)$ containing 0 and an open subset $U \subseteq O$ containing $x$ such that $B^{p} \times U \subseteq \Omega$ and $t\left(B^{p} \times U\right) \subseteq O$. Moreover, for any $y \in U$

$$
\exp _{y}: B^{p} \times\{y\} \rightarrow L_{y}
$$

is a diffeomorphism onto its image.

Since $(\Omega, \phi \cdot t, s)$ is adapted to the twisted path holonomy atlas, one has the corresponding quotient map

$$
\pi_{\Omega}: \Omega \rightarrow H(\mathcal{F}, \Gamma)
$$

such that $\pi_{\Omega}(0, x)=\pi_{W}(w)=g$.

Claim 2. If $\Omega=B^{p} \times U$, as given in Claim 1 , then $\pi_{\Omega}$ is injective.

We have to prove that $\forall(\lambda, y),(\mu, z) \in \Omega$

$$
\pi_{\Omega}(\lambda, y)=\pi_{\Omega}(\mu, z) \Longrightarrow(\lambda, y)=(\mu, z)
$$

Denoting with $s_{\mathcal{F}}^{\Gamma}$ the source map of the twisted holonomy groupoid $H(\mathcal{F}, \Gamma)$, one has by Proposition 3.2. c) in [1] and by 1.46 that

$$
y=s(\lambda, y)=s_{\mathcal{F}}^{\Gamma}\left(\pi_{\Omega}(\lambda, y)\right)=s_{\mathcal{F}}^{\Gamma}\left(\pi_{\Omega}(\mu, z)\right)=s(\mu, z)=z
$$

It remains to prove that $\pi_{\Omega}(\lambda, y)=\pi_{\Omega}(\mu, y) \Longrightarrow \lambda=\mu$. Again by Proposition 3.2. c) in [1], one also knows that $t_{\mathcal{F}}^{\Gamma} \circ \pi_{\Omega}=\phi \cdot t$. Therefore one has the following 
commutative diagram

$$
\begin{array}{ccc}
B^{p} \times\{y\} & \stackrel{\pi_{\Omega}}{\longrightarrow} & H(\mathcal{F}, \Gamma) \\
\downarrow \phi \cdot t & \circlearrowright & \downarrow t_{\mathcal{F}}^{\Gamma} \\
L_{y} & \stackrel{i d}{\longrightarrow} & L_{y}
\end{array}
$$

Now, it follows from Claim 1 that the vertical arrow $B^{p} \times\{y\} \rightarrow L_{y}$ is injective for any $y \in U$. This implies that $\pi_{\Omega}$ is injective.

Define a coordinate chart around $g$ in $H(\mathcal{F}, \Gamma)$ to be the image

$$
U(g)=\pi_{\Omega}(\Omega) \subseteq H(\mathcal{F}, \Gamma),
$$

where $\Omega$ is chosen as in Claim 1 .

Claim 3. $U(g)$ is an open set of $H(\mathcal{F}, \Gamma)$ with respect to the quotient topology.

We have to prove that, for all bi-submersions $\Omega_{i}$ in the twisted path holonomy atlas, $\pi_{i}^{-1}(U(g)) \subseteq \Omega_{i}$ is an open subset.

Consider a bi-submersion $\left(\Omega_{i}, t_{i}, s_{i}\right)$ in the twisted path holonomy atlas such that

$$
\pi_{i}^{-1}(U(g))=O \subseteq \Omega_{i} .
$$

Let $p \in O$ and consider $\pi_{i}(p) \in U(g)$. Since $\Omega \stackrel{\pi_{\Omega}}{\longrightarrow} U(g)$ is a bijection, there is a unique point $a \in \Omega$ such that $\pi_{\Omega}(a)=\pi_{i}(p)$.

By Corollary 1.3.3, there is an open neighborhood $O^{\prime} \subseteq \Omega_{i}$ containing $p$ and a morphism of bi-submersions

$$
f: O^{\prime} \subseteq \Omega_{i} \rightarrow \Omega
$$

such that $f(p)=a$. By Proposition $3.2 \mathrm{c}$ ) of [1], for all $q \in O^{\prime}$

$$
\pi_{\Omega}(f(q))=\pi_{i}(q)
$$

and, since $\pi_{\Omega}$ is bijective, it follows that

$$
\pi_{i}\left(O^{\prime}\right) \subseteq U(g)
$$

and

$$
O^{\prime} \subseteq O
$$

Therefore, each point $p \in O$ is internal and $O$ is an open set in $\Omega_{i}$. This proves that $U(g)$ is open in $H(\mathcal{F}, \Gamma)$ with respect to the quotient topology.

Now, we want to prove that the coordinate changes are diffeomorphisms. Let $g, g^{\prime} \in H(\mathcal{F}, \Gamma)$ and $U(g)$ and $U\left(g^{\prime}\right)$ coordinate charts around $g$ and $g^{\prime}$, respectively, such that $U(g) \cap U\left(g^{\prime}\right) \neq \emptyset$. Let $\gamma \in U(g) \cap U\left(g^{\prime}\right)$ with source 
$s_{\mathcal{F}}^{\Gamma}(\gamma)=p$ and target $t_{\mathcal{F}}^{\Gamma}(\gamma)=q$.

By construction, there exist two bi-submersions $\left(B_{\epsilon}^{p} \times U, t, s\right)$ and $\left(B_{\eta}^{p} \times V, t^{\prime}, s^{\prime}\right)$ such that

$$
\begin{array}{r}
\pi: B_{\epsilon}^{p} \times U \stackrel{\simeq}{\rightarrow} U(g) \\
\rho: B_{\eta}^{p} \times V \stackrel{\simeq}{\rightarrow} U\left(g^{\prime}\right)
\end{array}
$$

Since the open sets $U$ and $V$ are foliated charts, we have foliated coordinates on them, defined by the diffeomorphisms $\phi_{1}: B_{\epsilon^{\prime}}^{p} \times T \simeq U$ and, respectively, $\phi_{2}: B_{\eta^{\prime}}^{p} \times S \simeq V$. With a small abuse of notation, we will think of $U$ and $V$ as open subsets of $\mathbb{R}^{p} \times \mathbb{R}^{q}$ via these diffeomorphisms. Furthermore, we will denote the compositions $\pi \circ\left(i d \times \phi_{1}\right)$ and $\rho \circ\left(i d \times \phi_{2}\right)$, respectively, by $\pi$ and $\rho$ again. The coordinate change map is, now, given by

$$
\psi=\rho^{-1} \circ \pi: V_{1} \rightarrow V_{2}
$$

where $V_{1}=\pi^{-1}\left(U(g) \cap U\left(g^{\prime}\right)\right)$ and $V_{2}=\rho^{-1}\left(U(g) \cap U\left(g^{\prime}\right)\right)$.

The construction of the coordinate neighborhoods $U(g)$ and $U\left(g^{\prime}\right)$ shows that $s_{\mathcal{F}}^{\Gamma}(U(g))=U$ and $s_{\mathcal{F}}^{\Gamma}\left(U\left(g^{\prime}\right)\right)=V$ are foliated charts, whose intersection contains $s_{\mathcal{F}}^{\Gamma}(\gamma)=p$ and, analogously, $t_{\mathcal{F}}^{\Gamma}(U(g))$ and $t_{\mathcal{F}}^{\Gamma}\left(U\left(g^{\prime}\right)\right)$ are foliated charts, whose intersection contains $t_{\mathcal{F}}^{\Gamma}(\gamma)=q$.

Now, notice that $\psi$ commutes with the source and target maps on the bisubmersions. Indeed,

$$
s^{\prime} \circ \psi=\left(s_{\mathcal{F}}^{\Gamma} \circ \rho\right) \circ \psi=s_{\mathcal{F}}^{\Gamma} \circ \pi=s .
$$

The computation for the target is the same. Thus, one has the following commutative diagrams

$$
\begin{array}{ccc}
V_{1} & \stackrel{s}{\rightarrow} & s_{\mathcal{F}}^{\Gamma}(U(g)) \cap s_{\mathcal{F}}^{\Gamma}\left(U\left(g^{\prime}\right)\right) \\
\downarrow \psi & \circlearrowright & \| \\
V_{2} & \stackrel{s^{\prime}}{\rightarrow} & s_{\mathcal{F}}^{\Gamma}(U(g)) \cap s_{\mathcal{F}}^{\Gamma}\left(U\left(g^{\prime}\right)\right)
\end{array}
$$

and

$$
\begin{array}{cccc}
V_{1} & \stackrel{t}{\rightarrow} & t_{\mathcal{F}}^{\Gamma}(U(g)) \cap t_{\mathcal{F}}^{\Gamma}\left(U\left(g^{\prime}\right)\right) \\
\downarrow \psi & \circlearrowright & \| \\
V_{2} & \stackrel{t^{\prime}}{\rightarrow} & t_{\mathcal{F}}^{\Gamma}(U(g)) \cap t_{\mathcal{F}}^{\Gamma}\left(U\left(g^{\prime}\right)\right)
\end{array}
$$

Hence, if the coordinate change map between the foliated charts $s_{\mathcal{F}}^{\Gamma}(U(g))$ and $s_{\mathcal{F}}^{\Gamma}\left(U\left(g^{\prime}\right)\right)$ is given by $\phi(a, z)=(\alpha(a, z), \beta(z))$ and that between the foliated charts $t_{\mathcal{F}}^{\Gamma}(U(g))$ and $t_{\mathcal{F}}^{\Gamma}\left(U\left(g^{\prime}\right)\right)$ is $\theta(b, z)=(\delta(b, z), \beta(z))$, we deduce that

$$
\psi\left(\lambda_{1}, \mu_{1}, z\right)=\left(\delta\left(\lambda_{1}, z\right), \alpha\left(\mu_{1}, z\right), \beta(z)\right) .
$$

which shows that $\psi$ is a diffeomorphism between $V_{1}$ and $V_{2}$. This completes the proof of 1).

Let us now prove 2). From the definition of the coordinate charts, it is not difficult to realize that the source and the target maps $s_{\mathcal{F}}^{\Gamma}, t_{\mathcal{F}}^{\Gamma}: H(\mathcal{F}, \Gamma) \rightarrow M$ 
are smooth submersions. Indeed, for any $\gamma \in H(\mathcal{F}, \Gamma)$, one has a coordinate neighborhood $U(\gamma)$ around $\gamma$ diffeomorphic to $B_{\epsilon}^{p} \times B_{\epsilon^{\prime}}^{p} \times T$. The source and target maps restricted to $U(\gamma)$ take the form

$$
s_{\mathcal{F}}^{\Gamma}(\lambda, \mu, z)=(\mu, z)
$$

and

$$
t_{\mathcal{F}}^{\Gamma}(\lambda, \mu, z)=(\lambda, z)
$$

Analogously, the product of two composable elements $g_{1}=\left(\lambda_{1}, \mu_{1}, z\right)$ and $g_{2}=$ $\left(\mu_{1}, \mu_{2}, z\right)$ is given in coordinates by the formula

$$
\left(\lambda_{1}, \mu_{1}, z\right) \cdot\left(\mu_{1}, \mu_{2}, z\right)=\left(\lambda_{1}, \mu_{2}, z\right)
$$

For the unit map $u: M \rightarrow H(\mathcal{F}, \Gamma)$ and the inverse map $i: H(\mathcal{F}, \Gamma) \rightarrow H(\mathcal{F}, \Gamma)$ the proof is similar.

Remark 1.10. Considering, in particular, the trivial group $\Gamma=\{1\}$, we have explicitly proved that the holonomy groupoid $H(\mathcal{F})$ is a Lie groupoid of dimension $2 \operatorname{dim} \mathcal{F}+\operatorname{codim} \mathcal{F}$, as stated in 1.3 .6 .

Proposition 1.4.3. The holonomy groupoid $H(\mathcal{F})$ is an open Lie subgroupoid of $H(\mathcal{F}, \Gamma)$.

Proof. Let us consider the inclusion $j: H(\mathcal{F}) \rightarrow H(\mathcal{F}, \Gamma)$ as defined in the proof of Lemma 1.4.1. Let us first notice that $j$ is continuous with respect to the quotient topologies defined on $H(\mathcal{F})$ and $H(\mathcal{F}, \Gamma)$. This follows immediately from the commutative diagram

$$
\begin{array}{ccc}
\mathcal{U} & \hookrightarrow & \mathcal{U}^{\Gamma} \\
\pi \downarrow & \circlearrowright & \downarrow \pi_{\Gamma} \\
H(\mathcal{F}) & \stackrel{j}{\rightarrow} & H(\mathcal{F}, \Gamma)
\end{array}
$$

Furthermore, $j$ is a local diffeomorphism. Indeed, let $[u]$ be an element of $H(\mathcal{F})$ and consider $j([u])=[u]_{\Gamma} \in H(\mathcal{F}, \Gamma)$. Then, there is a coordinate neighborhood $U([u]) \subseteq H(\mathcal{F})$ of $[u]$ and a coordinate neighborhood $U\left([u]_{\Gamma}\right) \subseteq H(\mathcal{F}, \Gamma)$ of $[u]_{\Gamma}$ such that $j(U([u])) \subseteq U\left([u]_{\Gamma}\right)$. Since $s_{\mathcal{F}}^{\Gamma} \circ j=s_{\mathcal{F}}$, by Lemma 1.4.1), we deduce that $s_{\mathcal{F}}(U([u]))$ and $s_{\mathcal{F}}^{\Gamma}\left(U\left([u]_{\Gamma}\right)\right)$ are coordinate neighborhoods around the same point $s_{\mathcal{F}}^{\Gamma}\left([u]_{\Gamma}\right)=s_{\mathcal{F}}([u])$ such that $s_{\mathcal{F}}\left(U([u]) \subseteq s_{\mathcal{F}}^{\Gamma}\left(U\left([u]_{\Gamma}\right)\right)\right.$. Similarly, the formula $t_{\mathcal{F}}^{\Gamma} \circ j=t_{\mathcal{F}}$ implies that $t_{\mathcal{F}}(U([u])) \subseteq t_{\mathcal{F}}^{\Gamma}\left(U\left([u]_{\Gamma}\right)\right)$ and both are coordinate charts around the point $t_{\mathcal{F}}([u])$.

If the coordinates on $s_{\mathcal{F}}^{\Gamma}\left(U\left([u]_{\Gamma}\right)\right)$ are denoted by $(\mu, z)$ and those on $t_{\mathcal{F}}^{\Gamma}\left(U\left([u]_{\Gamma}\right)\right)$ by $(\lambda, z)$, we have that the coordinates on $U\left([u]_{\Gamma}\right)$ are given by $(\lambda, \mu, z)$. By restriction, each point of $U([u])$ is expressed by the same coordinates.

In these coordinates $j$ takes the form

$$
j(\lambda, \mu, z)=(\lambda, \mu, z) \in H(\mathcal{F}, \Gamma)
$$

for any point $(\lambda, \mu, z) \in H(\mathcal{F})$. Therefore, it is clearly smooth.

Moreover, one gets from the above expression that $j$ is an immersion for all 
$[u] \in H(\mathcal{F})$ and, thus, a local diffeomorphism, since the holonomy groupoid $H(\mathcal{F})$ and the twisted holonomy groupoid $H(\mathcal{F}, \Gamma)$ have the same dimension.

Now, knowing that $j: H(\mathcal{F}) \hookrightarrow H(\mathcal{F}, \Gamma)$ is a local diffeomorphism, it follows quite easily that $j(H(\mathcal{F})) \subseteq H(\mathcal{F}, \Gamma)$ is open. In fact, let us take any point $[v] \in j(H(\mathcal{F}))$. Then, there is a unique point $[u] \in H(\mathcal{F})$ such that $j([u])=[v]$. Since $j$ is a local diffeomorphism, there are open neighborhoods $U$ of $[u]$ in $H(\mathcal{F})$ and $V$ of $[v]$ in $H(\mathcal{F}, \Gamma)$ such that $j_{\mid U}: U \rightarrow V$ is a diffeomorphism. This shows that $V \subseteq j(H(\mathcal{F}))$ and, thus, that each point of $j(H(\mathcal{F}))$ is internal.

Recall that, for an arbitrary Lie groupoid $G \rightrightarrows M$, there exists always an open $s$-connected Lie subgroupoid of $G$, obtained by taking the connected components of the units with respect to the $s$-fibers. This open subgroupoid is called the $s$-connected component of the identities and it is denoted by $\mathfrak{C}^{s}(G)$. By the above proposition, one has therefore that $j(H(\mathcal{F})) \subseteq \mathfrak{C}^{s}(H(\mathcal{F}, \Gamma))$.

Let us now briefly recall the definition of the Lie algebroid $\mathfrak{g}$ associated to a Lie groupoid $G$ over $M$.

Since the source map $s: G \rightarrow M$ is a submersion, one considers the vector bundle $\operatorname{ker}(d s: T G \rightarrow T M)$ of $s$-vertical tangent vectors on $G$, i.e. all tangent vectors $v \in T G$ such that $d s(v)=0$. Its restriction along the unit map $u$ of the groupoid is denoted by $\mathfrak{g}$ and is a vector bundle on $M$. Equivalently, $\mathfrak{g}$ is the vector bundle $\bigcup_{x \in M} T_{1_{x}}\left(G_{x}\right)$ on $M$. The bundle morphism given by the differential $d t$ of the target map $t: G \rightarrow M$ is called the anchor map. Moreover, the space of smooth sections $C^{\infty}(M, \mathfrak{g})$ is naturally endowed with a Lie bracket. The triple $(\mathfrak{g},[\cdot, \cdot], d t)$ is the Lie algebroid of $G$. We will, more briefly, denote the Lie algebroid with $\mathfrak{g}$. For further properties concerning Lie algebroids, we refer to 39 and 38 .

A Lie groupoid $G$ over $M$ is called a foliation groupoid if its Lie algebroid $\mathfrak{g}$ has injective anchor map ( see [19]).

Theorem 1.4.4. The Lie groupoid $H(\mathcal{F}, \Gamma)$ over $M$ is a foliation groupoid with Lie algebroid isomorphic to the vector bundle $F=T \mathcal{F}$ tangent to the foliation. In particular, for every $x \in M$, the isotropy groups $H(\mathcal{F}, \Gamma)_{x}^{x}$ are discrete and the holonomy groups $H(\mathcal{F})_{x}^{x}$ are subgroups of $H(\mathcal{F}, \Gamma)_{x}^{x}$.

Proof. Let $\mathfrak{h}(\mathcal{F}, \Gamma)$ denote the Lie algebroid of $H(\mathcal{F}, \Gamma)$.

It is known that the Lie algebroid of the holonomy groupoid $H(\mathcal{F})$ is isomorphic to vector bundle $F=T \mathcal{F}$ on $M$ tangent to the foliation (see, for instance, 39, pag. 152, for a proof). Therefore, by a small abuse of notation, we will denote the Lie algebroid of $H(\mathcal{F})$ by $F$.

We want to prove that $\mathfrak{h}(\mathcal{F}, \Gamma) \cong F$.

Let $j: H(\mathcal{F}) \hookrightarrow H(\mathcal{F}, \Gamma)$. By Lemma 1.4.1, we know that $s_{\mathcal{F}}^{\Gamma} \circ j=s_{\mathcal{F}}$. This implies that

$$
d s_{\mathcal{F}}^{\Gamma} \circ d j=d s_{\mathcal{F}} .
$$

Then

$$
d j: \operatorname{ker}\left(d s_{\mathcal{F}}\right) \rightarrow \operatorname{ker}\left(d s_{\mathcal{F}}^{\Gamma}\right)
$$


Moreover, since $j \circ u_{\mathcal{F}}=u_{\mathcal{F}}^{\Gamma}$, one gets that

$$
j_{*}=d j_{\mid M}: F \rightarrow \mathfrak{h}(\mathcal{F}, \Gamma)
$$

is a morphism of Lie algebroid (see [38, section 3.5.)

Now, by Proposition $1.4 .3 j$ is a local diffeomorphism. Then, it follows that $j_{*}: F \rightarrow \mathfrak{h}(\mathcal{F}, \Gamma)$ must be a bundle isomorphism.

In particular, the Lie algebroid $\mathfrak{h}(\mathcal{F}, \Gamma)$ has injective anchor map, which means, by the characterisation theorem in [19], that $H(\mathcal{F}, \Gamma)$ is a foliation groupoid. This completes the proof.

One of the crucial features of the holonomy groupoid is its action on local transversal submanifolds. Indeed, each element $\gamma \in H(\mathcal{F}), \gamma: x \rightarrow y$ records a unique germ at $x$ of a locally defined diffeomorphism between two transverse submanifolds $T_{x}$ through $x$ and $T_{y}$ through $y$. This germ from $x$ to $y$ is the holonomy carried by the element $\gamma$ and any representative is called a holonomy transformation. Furthermore this action gives rise to an action of the holonomy groupoid on the normal bundle $\nu$ of the foliation, by taking the differential of the holonomy associated to every $\gamma \in H(\mathcal{F})$. We refer to [39, 11] and [12] for details and for many consequences this important notion.

Now, we want to show that this action of the holonomy groupoid, and its linearization, extends naturally to the twisted holonomy groupoid, giving rise to more general holonomies determined by the elements of the group $\Gamma$.

Theorem 1.4.5. Let $(M, \mathcal{F}, \Gamma)$ be a foliated $\Gamma$-manifold. For any two points $x, y \in M$ lying in the same leaf $L$ let us choose connected local transversals $T_{x}$ at $x$ and $T_{y}$ at $y$ and denote by Diff $f_{x}\left(T_{x}, T_{y}\right)$ the group of germs of local diffeomorphisms mapping $\left(T_{x}, x\right)$ to $\left(T_{y}, y\right)$. Then there exists a well defined map

$$
\Lambda_{T_{x}}^{T_{y}}: H(\mathcal{F}, \Gamma)_{x}^{y} \rightarrow \operatorname{Diff} f_{x}\left(T_{x}, T_{y}\right)
$$

such that

1. $\Lambda_{T_{x}}^{T_{z}}\left(\gamma_{1} \gamma_{2}\right)=\Lambda_{T_{y}}^{T_{z}}\left(\gamma_{1}\right) \Lambda_{T_{x}}^{T_{y}}\left(\gamma_{2}\right)$ for every $\gamma_{1}, \gamma_{2} \in H(\mathcal{F}, \Gamma)$ with $\gamma_{1}: z \rightarrow y$ and $\gamma_{2}: x \rightarrow y$. In particular, for every $x \in M$ the map

$$
\Lambda_{T_{x}}^{T_{x}}: H(\mathcal{F}, \Gamma)_{x}^{x} \rightarrow \operatorname{Diff} f_{x}\left(T_{x}, T_{x}\right)
$$

is a group homomorphism.

2. $\Lambda_{T_{x}}^{T_{y}}$ is injective for all $x, y \in M$.

Proof. Given connected local transversal submanifolds $T_{x}$ at $x$ and $T_{y}$ at $y$, we set $T=T_{x} \cup T_{y}$ and consider the subgroupoid $H(\mathcal{F}, \Gamma)_{T}^{T} \subseteq H(\mathcal{F}, \Gamma)$. By theorem 1.4.4 $H(\mathcal{F}, \Gamma)$ is a foliation groupoid in the sense of [19] and, consequently, one knows that $H(\mathcal{F}, \Gamma)_{T}^{T}$ is an étale groupoid (see [19], Lemma 2), i.e. the source and target maps restricted to $H(\mathcal{F}, \Gamma)_{T}^{T}$ are local diffeomorphisms. Therefore, for all $\gamma \in H(\mathcal{F}, \Gamma)_{x}^{y}$ we define

$$
\Lambda_{T_{x}}^{T_{y}}(\gamma)=\left(t \circ\left(s_{\mid U}\right)^{-1}\right)_{x}
$$


where $U$ is an arbitrary connected open neighborhood of $\gamma$ in the étale groupoid $H(\mathcal{F}, \Gamma)_{T}^{T}$ such that both $s_{\mid U}$ and $t_{\mid U}$ are diffeomorphisms onto suitable open sets of respectively $T_{x}$ and $T_{y}$.

Now, it is clear that the germ $\left(t \circ\left(s_{\mid U}\right)^{-1}\right)_{x}$ does not depend on the choice of $U$ and does only depend on $\gamma$.

Next, let us show that $\Lambda$ is compatible with the groupoid multiplication.

Let $x, y, z \in M$ and choose connected local transversals $T_{x}, T_{y}$ and $T_{z}$. We put $T=T_{x} \cup T_{y} \cup T_{z}$ and consider the corresponding étale groupoid $H(\mathcal{F}, \Gamma)_{T}^{T}$. Take now $\gamma_{1}, \gamma_{2} \in H(\mathcal{F}, \Gamma)_{T}^{T}$ with $\gamma_{1}: y \rightarrow z$ and $\gamma_{2}: x \rightarrow y$.

Since $H(\mathcal{F}, \Gamma)_{T}^{T}$ is étale, we can choose an open neighborhood $U_{1} \subseteq H(\mathcal{F}, \Gamma)_{T}^{T}$ of $\gamma_{1}$ such that both $s_{\mid U_{1}}$ and $t_{\mid U_{1}}$ are diffeomorphisms on their image. In the same way, we choose an open neighborhood $U_{2} \subseteq H(\mathcal{F}, \Gamma)_{T}^{T}$ of $\gamma_{2}$ such that the restrictions $s_{\mid U_{2}}$ and $t_{\mid U_{2}}$ are diffeomorphisms on the image. Since $s\left(\gamma_{1}\right)=t\left(\gamma_{2}\right)$, up to shrinking $U_{1}$ and $U_{2}$ we can assume that $s\left(U_{1}\right)=t\left(U_{2}\right)$. Thus, by construction each element of $U_{2}$ can be composed with exactly one element of $U_{1}$ and the resulting subset

$$
U=\left\{\eta_{1} \eta_{2} \mid \eta_{1} \in U_{1}, \eta_{2} \in U_{2} \text { with } s\left(\eta_{1}\right)=t\left(\eta_{2}\right)\right\}
$$

contains the product $\gamma_{1} \gamma_{2}$. Furthermore, both $s_{\mid U}$ and $t_{\mid U}$ are diffeomorphisms on their image.

Hence, one has that

$$
\left(s_{\mid U}\right)^{-1}=\left(s_{\mid U_{1}}\right)^{-1} \circ t \circ\left(s_{\mid U_{2}}\right)^{-1}
$$

which immediately implies that

$$
\Lambda_{T_{z}}^{T_{x}}\left(\gamma_{1} \gamma_{2}\right)=\Lambda_{T_{y}}^{T_{z}}\left(\gamma_{1}\right) \Lambda_{T_{x}}^{T_{y}}\left(\gamma_{2}\right)
$$

It remains to show the injectivity. We need only to prove that the group homomorphism $\Lambda_{T_{x}}^{T_{x}}$ is injective for all $x \in M$, that is if for $\gamma \in H(\mathcal{F}, \Gamma)_{x}^{x}$, $\Lambda_{T_{x}}^{T_{x}}(\gamma)=i d_{x}$, then $\gamma=1_{x}$. Here we have denoted by $i d_{x}$ the identity of Dif $f_{x}\left(T_{x}\right)$ and by $1_{x} \in H(\mathcal{F}, \Gamma)_{x}^{x}$ the unity arrow at $x$.

In other words, we must show that if there exists an open connected neighborhood $V^{\prime}$ of $H(\mathcal{F}, \Gamma)_{T_{x}}^{T_{x}}$ containing $\gamma$ such that $s\left(V^{\prime}\right)=t\left(V^{\prime}\right)=V \subseteq T_{x}$ and $t \circ\left(s_{\mid V}\right)^{-1}=i d_{V}$, then there is a bi-submersion $(U, t, s)$ adapted to the twisted path holonomy atlas and which contains a point $u \in U$ with $[u]=\gamma$ and a local bisection $E$ through $u$ carrying the identity (cf. Corollary 1.3 .3 and Theorem 1.3.4.

For notational simplicity, let us denote the transverse local diffeomorphism $t \circ\left(s_{\mid V}\right)^{-1}$ representing $\Lambda_{T_{x}}^{T_{x}}(\gamma)$ simply by $f$.

Up to shrinking $V$ if needed, we can choose an open coordinate neighborhood $U(\gamma)$ of $\gamma$ in the whole groupoid $H(\mathcal{F}, \Gamma)$ diffemorphic to $W^{\prime} \times{ }_{f} W$, where $W \simeq$ $D \times S$ and $W^{\prime} \simeq D^{\prime} \times S$ are two foliated charts around $x$ both containing $V$ as transversal submanifold and $W^{\prime} \times{ }_{f} W=\left\{\left(\left(w^{\prime}, s^{\prime}\right),(w, s)\right) \in W^{\prime} \times W \mid s^{\prime}=f(s)\right\}$. Hence, $W^{\prime} \times{ }_{f} W$ is naturally diffeomorphic to $D^{\prime} \times D \times S$ and $V \simeq S$, the diffeomorphism being induced by the transverse coordinate. 
Moreover, the restrictions $s_{\mid U(\gamma)}$ and $t_{\mid U(\gamma)}$ can be identified, respectively, with the projections $p_{1}: W^{\prime} \times_{f} W \rightarrow W$ and $p_{2}: W^{\prime} \times_{f} W \rightarrow W^{\prime}$. Therefore $\left(W^{\prime} \times{ }_{f} W, p_{2}, p_{1}\right)$ is a bi-submersion adapted to the twisted holonomy atlas representing $\gamma$ through the point $p=\left(\left(b_{0}, s_{0}\right),\left(b_{0}, s_{0}\right)\right)$.

Now, since by assumption $f$ is the identity map, then $W^{\prime} \times{ }_{f} W=\left\{\left(\left(w^{\prime}, s^{\prime}\right),(w, s)\right) \in\right.$ $\left.W^{\prime} \times W \mid s^{\prime}=s\right\}$, which means that the source and target of any point $p \in$ $W^{\prime} \times{ }_{f} W$ must lie in the same plaque. Hence, taking an open subset $B \times S \subseteq$ $\left(D^{\prime} \times S\right) \cap(D \times S)$ which contains $x=\left(b_{0}, s_{0}\right)$, we deduce that the set

$$
\Delta_{B \times S}=\left\{((b, s),(b, s)) \in W^{\prime} \times_{f} W \mid b \in B, s \in S\right\}
$$

is a bisection through $p$ that carries the identity. This proves the claim and concludes the proof of the theorem.

Let $x, y \in M$ as above. Let us take two connected local transversals $T_{x}$ and $T_{x}^{\prime}$ at $x$ both contained in a foliated chart around $x$ and two connected transversals $T_{y}$ and $T_{y}^{\prime}$ at $y$ both contained in a foliated chart around $y$.

If you denote by $\phi: T_{x} \rightarrow T_{x}^{\prime}$ and by $\psi: T_{y} \rightarrow T_{y}^{\prime}$ the diffeomorphisms given by "being on the same plaque", then for $\gamma \in H(\mathcal{F}, \Gamma)_{x}^{y}$

$$
\Lambda_{T_{x}^{\prime}}^{T_{y}^{\prime}}(\gamma)=\psi_{y} \circ \Lambda_{T_{x}}^{T_{y}}(\gamma) \circ\left(\phi_{x}\right)^{-1} .
$$

In this sense the map $\Lambda$ defined in Theorem 1.4.5 does not depend on the choice of the transversals. In the following, we will therefore denote $\Lambda_{T_{x}}^{T_{y}}$ simply by $\Lambda$ assuming implicitly that two transversal submanifolds respectively at $x$ and $y$ have been chosen.

It is immediate to see that the map $\Lambda$ defined above for the twisted holonomy groupoid extends the natural transverse action of the holonomy groupoid.

Definition 1.28. Given any $\gamma \in H(\mathcal{F}, \Gamma), \gamma: x \rightarrow y$, the germ $\Lambda(\gamma)$ as defined in Theorem 1.4 .5 is called the holonomy along $\gamma$.

Recall that to any vector bundle $(E \stackrel{\pi}{\rightarrow} M)$ one can associate its frame linear groupoid, namely the groupoid

$$
\Phi(E)=\left\{(x, \phi, y) \mid x, y \in M, \phi: E_{x} \rightarrow E_{y} \text { is a linear isomorphism }\right\} .
$$

A linear action of a Lie groupoid $G$ over $M$ on the vector bundle $(E \stackrel{\pi}{\rightarrow} M)$ is by definition a morphism of Lie groupoids

$$
\rho: G \rightarrow \Phi(E)
$$

which covers the identity of $M$. For more about this point, see [38].

An important consequence of Theorem 1.4.5 is the following corollary.

Corollary 1.4.6. If $x, y$ are two points of a foliated $\Gamma$-manifold $(M, \mathcal{F}, \Gamma)$ lying in the same leaf $L$ and $\nu$ is the normal bundle of the foliation, then for every $\gamma \in H(\mathcal{F}, \Gamma), \gamma: x \rightarrow y$, one has a linear isomorphism

$$
\lambda(\gamma): \nu_{x} \rightarrow \nu_{y}
$$


induced by the differential $(d \Lambda(\gamma))_{x}$ of the holonomy $\Lambda(\gamma)$. This determines a linear action

$$
\lambda: H(\mathcal{F}, \Gamma) \rightarrow \Phi(\nu)
$$

of $H(\mathcal{F}, \Gamma)$ on $\nu$, where $\Phi(\nu)$ is the frame linear groupoid of $\nu$.

Proof. Choose any two connected transversal submanifolds $S_{x}$ at $x$ and $S_{y}$ at $y$. By transversality one has the linear isomorphisms $\rho: T_{x}\left(S_{x}\right) \rightarrow \nu_{x}$ and $\mu: T_{y}\left(S_{y}\right) \rightarrow \nu_{y}$, induced by the decomposition $T_{x} M=T_{x}(\mathcal{F}) \oplus T_{x}\left(S_{x}\right)$ and $T_{y} M=T_{y}(\mathcal{F}) \oplus T_{y}\left(S_{y}\right)$. We set

$$
\lambda(\gamma)=\mu \circ(d \Lambda(\gamma))_{x} \circ \rho^{-1}
$$

It is immediate to see that $\lambda(\gamma)$ is well defined, i.e. it does not depend on the choice of the transversals.

Moreover, since by Theorem $1.4 .5 \Lambda$ is compatible with the groupoid multiplication, $\lambda: H(\mathcal{F}, \Gamma) \rightarrow \Phi(\nu)$ determines a morphism of Lie groupoids, giving rise to a linear action of $H(\mathcal{F}, \Gamma)$ on $\nu$.

The linear action $\lambda$ of $H(\mathcal{F}, \Gamma)$ on the normal bundle $\nu$ is very important and will play a central role in Chapter 4

\subsection{Lie $\Gamma$-groupoids and inner automorphisms}

In the present section we will see that a $\Gamma$-foliated manifold $(M, \mathcal{F}, \Gamma)$ induces naturally an additional structure on its holonomy groupoid and twisted holonomy groupoid. Indeed, the action of $\Gamma$ on $(M, \mathcal{F})$ "integrates" to an action by Lie groupoid morphisms on the groupoids of the foliation, giving rise to Lie $\Gamma$-groupoids.

We will give definitions and some properties of this general concept. In particular, we will consider actions on Lie groupoids given by inner automorphisms, which are particulartly suitable for our purposes. The most important result of this section and our motivation for introducing twisted holonomy groupoids is that, given any $\Gamma$-foliated manifold, the action induced on its twisted holonomy groupoid is always given by inner automorphisms, while the induced action on the holonomy groupoid may be more complicated.

Let us begin with general definitions about Lie $\Gamma$-groupoids.

Let $G$ be a Lie groupoid with space of objects $G^{(0)}$ and let $\Gamma$ be a Lie group.

Definition 1.29. A (left) Lie $\Gamma$-groupoid is a Lie groupoid $G$ endowed with a smooth (left) $\Gamma$-action $\varrho$ on its space of arrows that commutes with all structure maps of the groupoid. We will also denote a Lie $\Gamma$-groupoid by $(G, \Gamma, \varrho)$, specifying explicitly the action.

It is evident from the definition that each element $h \in \Gamma$ acts on the Lie $\Gamma$-groupoid $G$ as a morphism of Lie groupoids, i.e. as a functor $\varrho(h): G \rightarrow G$ which is a smooth map both on objects and on arrows. 
Example 1.10. Perhaps the most basic example of Lie $\Gamma$-groupoid is provided by the smooth action of a Lie group $\Gamma$ on a smooth manifold $M$, viewed as trivial groupoid.

Another very natural example is the following. If $\Gamma$ and $K$ are both Lie groups and $\Gamma$ acts on $K$ by $\alpha: \Gamma \rightarrow A u t(K)$, then $K$ can be viewed as a Lie $\Gamma$-groupoid.

Lie $\Gamma$-groupoids, in particular those arising in the theory of foliations, will be our main objects of study. For many purposes we have in mind, we will not deal with general actions on Lie groupoids, but, rather, we will consider special ones.

To give an idea of how these actions are, let us consider first the case of Lie groups.

If $\Gamma$ and $K$ are two Lie groups and if there exists a Lie group homomorphism $\delta: \Gamma \rightarrow K$, a particularly simple action of $\Gamma$ on $K$ is that induced by $\delta$ and given by the formula

$$
\begin{array}{r}
\alpha: \Gamma \rightarrow \operatorname{Aut}(K) \\
h \mapsto \alpha_{h}(k)=\delta(h) k \delta\left(h^{-1}\right)
\end{array}
$$

Viewing $K$ as groupoid, we have therefore a Lie $\Gamma$-groupoid.

Even simpler, let $K$ denote, again, a Lie group and consider the action of $K$ on itself by conjugation. Looking at it as a groupoid with space of objects $K^{(0)}=\{e\}$, one can interpret this action in a slightly different way. Indeed, let us think of left translations of the group, i.e. the diffeomorphisms $L_{g}: k \mapsto g k$, as the smooth maps

$$
\begin{aligned}
\phi_{g}: K^{(0)} & \rightarrow K \\
e & \mapsto g
\end{aligned}
$$

defined on the space of objects of the groupoid $K$. Since there is only one object, namely the identity $e \in K$, there is an obvious bijection $\phi_{g} \mapsto g$ and the action on $K$ by conjugation can be rewritten in terms of the $\phi_{g}, \forall g \in K$,

$$
K \rightarrow \operatorname{Aut}(K) \quad g \mapsto \phi_{g}(e) k \phi_{g^{-1}}(e)=g k g^{-1}
$$

where we think of the group element $k$ as an arrow $k: e \rightarrow e$.

We will mainly deal with actions of Lie groups on Lie groupoids that are formally defined as in the equation above.

The first difficulty, however, one encounters in generalizing the above construction to general Lie groupoids is that left translations of Lie groupoids are only defined along the fibers of the source and target maps, while we need maps of the whole groupoid. To overcome this problem, we define the following notion.

Definition 1.30. Let $G$ be a Lie groupoid with space of objects $G^{(0)}$. A bisection of the groupoid is a smooth map $\phi: G^{(0)} \rightarrow G$ which is a right inverse of the source map and such that $t \circ \phi$ is a diffeomorphism of $G^{(0)}$. The set of bisections of $G$ is denoted by $\operatorname{Bis}(G)$. 
Bisections can be regarded somehow as generalized elements of the groupoids, since, in some part of the theory, they play the role that, in the case of groups, is taken by the group elements themselves.

Remark 1.11. Let $G$ be a Lie groupoid over $G^{(0)}$ and let $\sigma: G^{(0)} \rightarrow G$ be a bisection. The image

$$
V=\left\{\sigma(x) \mid x \in G^{(0)}\right\} \subseteq G
$$

is a closed embedded submanifold of $G$ such that the restrictions of both $s$ and $t$ to $V \rightarrow G^{(0)}$ are diffeomorphisms. Conversely, if there is a closed embedded submanifold $V \subseteq G$ so that the restrictions $s, t: V \rightarrow G^{(0)}$ are both diffeomorphisms, then $V$ is the image of a unique bisection.

This shows that the notion of bisection given above for Lie groupoids coincides with the notion of bisections given in 1.22 for bi-submersions.

We will, sometimes, identify a bisection with its image.

Given a bisection $\phi \in \operatorname{Bis}(G)$ we define the diffeomorphism

$$
\begin{array}{r}
L_{\phi}: G \rightarrow G \\
g \mapsto \phi(t(g)) \cdot g
\end{array}
$$

These maps will play an important role in the following.

Let us now list some basic properties of bisections, whose proof is straightforward.

Lemma 1.5.1. Let $G$ be a Lie groupoid over $G^{(0)}$.

1. The set Bis $(G)$ of bisections of $G$ is a group with respect to the multiplication defined by

$$
(\phi \star \psi)(x)=\phi((t \circ \psi)(x)) \psi(x)
$$

for all $x \in G^{(0)}$.

2. One has that $L_{\phi \star \psi}=L_{\phi} \circ L_{\psi}$.

3. The map $\phi \mapsto t \circ \phi$ is a group homomorphism from Bis $(G)$ to the diffeomorphisms of $G^{(0)}$ that preserve the orbits of the natural action of $G$ on $G^{(0)}$.

Since $\operatorname{Bis}(G)$ is a group, any group homomorphism $\sigma: \Gamma \rightarrow \operatorname{Bis}(G)$ induces an action of $\Gamma$ on the Lie groupoid $G$, making it into a Lie $\Gamma$-groupoid. Indeed, the map $F_{h}: G \rightarrow G$ defined by

$$
F_{h}(g)=\sigma_{h}(t(g)) g \sigma_{h}(s(g))^{-1}
$$

is clearly a Lie groupoid morphism over the diffeomorphism $t \circ \sigma_{h}$.

Definition 1.31. Let $G$ be a Lie groupoid. 
1. A morphism of Lie groupoids $F: G \rightarrow G$ is said to be an inner automorphism, if there exists a bisection $\phi \in \operatorname{Bis}(G)$ such that

$$
F(g)=\phi(t(g)) \cdot g \cdot \phi(s(g))^{-1}
$$

2. Let $(G, \Gamma, \varrho)$ be a Lie $\Gamma$-groupoid. The action is given by inner automorphisms, if there exists a group homomorphism $\sigma: \Gamma \rightarrow B i s(G)$ that induces $\varrho$ on $G$.

Note that an inner automorphism $F_{\phi}$ is an isomorphism of Lie groupoids over the objects map $t \circ \phi$.

Thus, if the action on the groupoid $G$ is defined through the group homomorphism $\sigma: \Gamma \rightarrow \operatorname{Bis}(G)$, for any $h \in \Gamma$, an action on the space of objects $G^{(0)}$ is induced by letting $h \in \Gamma$ act through the diffeomorphism $t \circ \sigma_{h}$. For simplicity, we will denote this diffeomorphism simply by $h$. Moreover, we will say that the bisection $\sigma_{h}$ lifts the diffeomorphism $h$ to stress that $t \circ \sigma_{h}=h$.

Remark 1.12. Let $F_{\phi}$ be an inner automorphism defined by the bisection $\phi \in B i s(G)$. Then $\phi$ can be thought of as a natural transformation between the functor $F_{\phi}$ and the identity functor. Indeed, $\phi$ assigns to each object in $G$ a morphism of this category. Moreover for any $\gamma \in G, \gamma: x \rightarrow y$, one gets by definition that

$$
F_{\phi}(\gamma) \phi(x)=\phi(y) \gamma,
$$

which shows that $\phi$ defines a natural transformation between $F_{\phi}$ and the identity functor.

Thus, we use the structure of 2-category of the groupoid $G$.

We now return to the case of foliated manifolds.

Let $(M, \mathcal{F})$ be a foliated manifold and pick an element $f \in \operatorname{Diff}(\mathcal{F})$. We have seen in 1.2 .1 that $f$ must preserve the equivalence relation $\mathcal{R}_{\mathcal{F}}$ of the foliation. In other words, $f$ induces a functor

$$
F: \mathcal{R}_{\mathcal{F}} \rightarrow \mathcal{R}_{\mathcal{F}}
$$

that associates the object $f(x)$ to any $x \in \operatorname{Obj}\left(\mathcal{R}_{\mathcal{F}}\right)=M$ and to any morphism $(x, y) \in \mathcal{R}_{\mathcal{F}}$ associates a new morphism $F(x, y)=(f(x), f(y)) \in \mathcal{R}_{\mathcal{F}}$.

Besides one has the following proposition

Proposition 1.5.2. Any element $f \in \operatorname{Diff}(\mathcal{F})$ induces a functor $F$ on the holonomy groupoid $H(\mathcal{F})$ of the foliation $(M, \mathcal{F})$. This functor is naturally an isomorphism of Lie groupoids.

Before proving this proposition, let us observe the following simple but interesting feature of $f$. Since $f \in \operatorname{Diff}(\mathcal{F})$, choosing suitable foliated charts $\left(U_{1}, y_{1}\right)$ around any $p \in M$ and $\left(U_{2}, y_{2}\right)$ around $f(p)$ (here, we have just written down the submersions $y_{i}$ given by transverse coordinates. See Remark 1.1 for details), such that $f:\left(U_{1}, y_{1}\right) \rightarrow\left(U_{2}, y_{2}\right)$, one immediately gets that $f$ is a 
bundle morphism, i.e. it preserves the plaques. Thus, we have the transverse local diffeomorphism

$$
\operatorname{Hol}(f): y_{1}\left(U_{1}\right) \rightarrow y_{2}\left(U_{2}\right)
$$

which records the local action of $f$ on the transversals $y_{1}\left(U_{1}\right)$ and $y_{2}\left(U_{2}\right)$.

Proof. Using the description of the holonomy groupoid given by leafwise paths (see 1.26), let us define the functor $F: H(\mathcal{F}) \rightarrow H(\mathcal{F})$ induced by $f$ in the following way: choose an element $\gamma \in H(\mathcal{F})$ represented by the leafwise path $\alpha:[0,1] \rightarrow M$ and set $F(\gamma)=[f \circ \alpha]$.

We have to prove that this definition is well-posed. Thus, take another representative for $\gamma$ given by the leafwise path $\beta:[0,1] \rightarrow M$. Since $\alpha$ and $\beta$ define the same element in $H(\mathcal{F})$, then their holonomies $h_{\alpha}, h_{\beta}$, respectively, along $\alpha$ and $\beta$, coincide on a suitable open neighborhood of $x=s(\gamma)$ in a local transversal $T_{x}$.

Since $f \in \operatorname{Diff}(\mathcal{F})$, one gets that

$$
h_{f \circ \alpha}=H o l(f) \circ h_{\alpha} \circ H o l(f)^{-1}
$$

on a suitable open neighborhood of the transversal $\operatorname{Hol}(f)\left(T_{x}\right)$ at $f(x)$. Then, since locally $h_{\alpha}=h_{\beta}$, it follows that

$$
h_{f \circ \alpha}=h_{f \circ \beta}
$$

on a suitable neighborhhood of $f(x)$ in the transversal. This shows that $F$ is well-defined.

From its definition it is clear that the map $F$ associated to $f$ defines a functor of $H(\mathcal{F})$, that has an inverse, namely the functor $F^{\prime}$ associated to $f^{-1}$. Finally, for every $\gamma \in H(\mathcal{F})$ there are coordinate charts $U(\gamma) \simeq D \times D \times S$ around $\gamma$ and $U(F(\gamma)) \simeq D^{\prime} \times D^{\prime} \times S^{\prime}$ around $F(\gamma)$ such that $F(U(\gamma)) \subseteq U(F(\gamma))$ and the map $F$ takes the form

$$
F(a, b, t)=\left(f_{2}(a, t), f_{1}(b, t), \operatorname{Hol}(f)(t)\right)
$$

where $f_{\mid s(U(\gamma))}(b, t)=\left(f_{1}(b, t), \operatorname{Hol}(f)(t)\right)$ and $f_{\mid t(U(\gamma))}(a, t)=\left(f_{2}(a, t), H o l(f)(t)\right)$. Hence, $F$ is smooth and determines an isomorphism of Lie groupoids.

Corollary 1.5.3. Let $(M, \mathcal{F}, \Gamma)$ be a foliated $\Gamma$-manifold. Then its holonomy groupoid $H(\mathcal{F})$, with the induced $\Gamma$-action given by Proposition 1.5.2. is a Lie $\Gamma$-groupoid.

Now, in the case of a foliated manifold $(M, \mathcal{F})$ and for an arbitrary $h \in$ $\operatorname{Diff}(\mathcal{F})$, one may ask whether the induced Lie groupoid isomorphism $F_{h}$ of $H(\mathcal{F})$ is an inner automorphism.

As suggested by equation 1.80 , this is equivalent to ask whether there exists a bisection $\phi_{h} \in \operatorname{Bis}(H(\mathcal{F}))$ such that $t \circ \phi_{h}=h$ and the holonomy along $\phi_{h}(x)$, for all $x \in M$, coincides with the transverse local diffeomorphism $\mathrm{Hol}(h)$ on a suitable transverse open neighborhood of $x$.

In some case, this happens, as the following example shows. 
Example 1.11. If the holonomy of each leaf of the foliation $(M, \mathcal{F})$ is trivial, for instance, for a simple foliation, we know that the holonomy groupoid coincides with the equivalence relation $\mathcal{R}_{\mathcal{F}}$. See Example 1.9 . Thus, considering $\mathcal{R}_{\mathcal{F}}$, one has a natural bisection given by the graph of $f$, i.e. more precisely

$$
\begin{gathered}
\phi_{f}: M \rightarrow \mathcal{R}_{\mathcal{F}} \\
p \rightarrow(p, f(p)) .
\end{gathered}
$$

This map is smooth and the element $(p, f(p))$ coincides with the $H o l(f)$ at $p$ for trivial reasons.

However, it is not true, in general, that for any diffeomorphism $f \in \operatorname{Diff}(\mathcal{F})$ of the foliated manifold $(M, \mathcal{F})$ there is a bisection $\phi_{f} \in \operatorname{Bis}(H(\mathcal{F}))$ with values in the holonomy groupoid $H(\mathcal{F})$ satisfying the extra conditions indicated above. The following example has been pointed out to us by Moulay Benameur and James Heitsch.

Example 1.12. Let $S^{1}$ be the 1 -sphere and consider a one-parameter group of diffeomorphisms from the south pole to the north pole, induced by a suitable vector field $X \in C^{\infty}\left(T S^{1}\right)$ that vanishes at the north and at the south poles. Let $f: S^{1} \rightarrow S^{1}$ be the time-1 diffeomorphism of this flow and define the natural action of $\mathbb{Z}$ on $S^{1}$ induced by $f$. Moreover, let $\mathbb{Z}$ act on $\mathbb{R}$ by integer translations. Now, following the recipe explained in Example 1.3 and observing that $\mathbb{R} \times S^{1} / \mathbb{Z} \simeq T^{2}$, the 2 -dimensional torus, we get the foliated bundle $\left(T^{2}, \pi, \mathcal{F}\right)$, which has two leaves which are $S^{1} \mathrm{~s}$, corresponding to the fixed points of $f$, and all the other leaves are copies of $\mathbb{R}$ which wind from the south $S^{1}$ to the north $S^{1}$. Now, consider the diffeomorphism of $T^{2}$ which on the east part is the identity, but on the west part is given by going along each leaf a distance so that the induced map on the boundary $S^{1}$ leaves is the identity. This cannot be a holonomy diffeomorphism because the holonomy of the $S^{1}$ leaves is two sided, while the local transverse diffeomorphism $\mathrm{Hol}(f)$ induced by $f$ is a map which is the identity on one side and a contraction or expansion on the other.

Now, there are (at least) two ways to solve this problem. First, one could just consider those leafwise diffeomorphisms, that have the above properties. To this regard, we present the following notion, given by Benameur and Heitsch in [7.

Definition 1.32. Let $(M, \mathcal{F})$ a foliated manifold and $f \in \operatorname{Diff}(\mathcal{F})$. We say that $f$ is a holonomy diffeomorphism, if there exists a bisection $\phi_{f} \in \operatorname{Bis}(H(\mathcal{F}))$ such that

1. $\phi_{f}$ lifts $f$, i.e. $t \circ \phi_{f}=f$

2. for every $x \in M$, the holonomy along $\phi_{f}(x)$ coincides with the germ of the transverse local diffeomorphism $\operatorname{Hol}(f)$ at $x$.

Remark 1.13. If $f$ is a holonomy diffeomorphism, then the bisection $\phi$ associated to $f$ must be unique. 
Recall that two leaf-preserving diffeomorphisms $f, g \in \operatorname{Diff}(\mathcal{F})$ are leafwise homotopic, in symbols $f \sim_{\mathcal{F}} g$, if there exists a leafwise homotopy between them as defined in Definition 1.13 .

Lemma 1.5.4. ([7]) Let $(M, \mathcal{F})$ be a foliated manifold.

1. Every element $f \in \exp (\mathcal{F})$ is a holonomy diffeomorphism.

2. More generally, if $f \in \operatorname{Diff}(\mathcal{F}), g \in \operatorname{Diff}(\mathcal{F})$ is a holonomy diffeomorphism and $f \sim_{\mathcal{F}} g$, then also $f$ is a holonomy diffeomorphism. In particular, every $f \in$ Dif $f_{0}(\mathcal{F})$ is a holonomy diffeomorphism.

3. If $(M, \mathcal{F}, \Gamma)$ is a foliated $\Gamma$-manifold with $\Gamma$ a connected Lie group, then each element of $\Gamma$ is a holonomy diffeomorphism.

The proof of the following lemma is straightforward.

Lemma 1.5.5. Let $(M, \mathcal{F})$ be a foliated manifold. Let $f$ be a holonomy diffeomorphism, with induced Lie groupoid morphism on $H(\mathcal{F})$ denoted by $F$. Then, for any $\gamma \in H(\mathcal{F}), \gamma: x \rightarrow y$, one has that the diagram

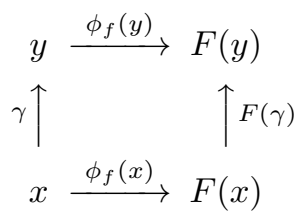

is commutative. In other words the bisection $\phi_{f}$ is a natural transformation between the functor $F$ and the identity functor.

A second possible way to solve the above problem consists in considering, instead of the holonomy groupoid, another groupoid, whose elements carry the germs of the transverse local diffeomorphisms $\mathrm{Hol}(f)$ defined by $f$. This groupoid is by construction provided by the twisted holonomy groupoid.

More precisely, one gets the following results. These have been our main motivation to introduce twisted holonomy groupoids.

Theorem 1.5.6. Let $(M, \mathcal{F})$ be a foliated manifold and let $f \in \operatorname{Diff}(\mathcal{F})$ be any leaf-preserving diffeomorphism. Let us build the holonomy groupoid $H(M, \mathcal{F}, \mathbb{Z})$ twisted by the subgroup $\mathbb{Z} \leq \operatorname{Diff}(\mathcal{F})$ generated by $f$. Then, for any element $n \in \mathbb{Z}$, there exists a bisection $\phi_{n}: M \rightarrow H(M, \mathcal{F}, \mathbb{Z})$ such that

1. $\phi_{n}$ lifts $f^{n}$, i.e. $t \circ \phi_{n}=f^{n}$;

2. for any $x \in M$, the holonomy along $\phi_{n}(x)$ coincides with the germ $\operatorname{Hol}(f)_{x}$ at $x$ of the transverse local action induced by $f$.

Moreover, the correspondence $n \mapsto \phi_{n}$ is a group homomorphism $\mathbb{Z} \rightarrow \operatorname{Bis}(H(M, \mathcal{F}, \mathbb{Z}))$.

Thus, by means of the bisections $\phi_{n}$ we can define an action of $\mathbb{Z}$ by inner automorphisms on the twisted holonomy groupoid $H(M, \mathcal{F}, \mathbb{Z})$, as in Equation 1.76 .

The above theorem is actually a special case of the next result, valid for an arbitrary Lie group $\Gamma$ and any foliated $\Gamma$-manifold. 
Theorem 1.5.7. Let $(M, \mathcal{F}, \Gamma)$ be a foliated $\Gamma$-manifold and consider its twisted holonomy groupoid $H(M, \mathcal{F}, \Gamma)$. Then, for any $h \in \Gamma$ there exists a bisection $\phi_{h} \in \operatorname{Bis}(H(M, \mathcal{F}, \Gamma))$ satisfying

1. $t \circ \phi_{h}=h$;

2. for any $x \in M$, the holonomy along $\phi_{h}(x)$ coincides with the germ $\operatorname{Hol}(f)_{x}$ at $x$ of the transverse local action $\mathrm{Hol}(h)$.

Moreover, the map $h \mapsto \phi_{h}$ from $\Gamma$ to $\operatorname{Bis}(H(\mathcal{F}, \Gamma))$ is a group homomorphism.

Proof. Let $h \in \Gamma$ and choose an arbitrary point $x \in M$. By definition of the twisted path holonomy atlas (see 1.42 there exists a bi-submersion $\left(U, t_{U}, s_{U}\right)$ in the twisted path holonomy atlas and a point $u \in U$ such that

- $s_{U}(u)=x$ and,

- there is a bisection $c: A \rightarrow U$ defined on a suitable open neighborhood of $x$ in $M$ such that $c(x)=u$ and the local diffeomorphism associated to it coincides with the restriction of $h$ on $A$.

We define

$$
\phi_{h}(x)=\pi_{U}(c(x))=[u] \in H(\mathcal{F}, \Gamma),
$$

where $\pi_{U}: U \rightarrow H(\mathcal{F}, \Gamma)$ is the quotient map of $U$ (cf. Theorem 1.3.4).

It is clear from the definition that $s\left(\phi_{h}(x)\right)=s_{U}(u)=x$ and $t\left(\phi_{h}(x)\right)=t_{U}(u)=$ $h(x)$.

Furthermore, the element $\phi_{h}(x)$ is well defined. In fact, if there is another bi-submersion $\left(W, t_{W}, s_{W}\right)$ adapted to the twisted path holonomy atlas and a point $w \in W$ such that $s_{W}(w)=x$ and there is a bisection $c^{\prime}: B \rightarrow W$ with $c^{\prime}(x)=w$ and whose associated diffeomorphism is the restriction of $h$ on $B$, then

$$
\pi_{U}(u)=\pi_{W}(w)=\phi_{h}(x)
$$

since $u$ and $w$ carry the same diffeomorphism (see Corollary 1.3.3).

Since the twisted path holonomy atlas covers $M, \phi_{h}(x)$ is defined for all $x \in M$ and it gives rise to a map

$$
\phi_{h}: M \rightarrow H(\mathcal{F}, \Gamma)
$$

such that $s \circ \phi_{h}=i d_{M}$ and $t \circ \phi_{h}=h$.

Moreover, this map $\phi_{h}$ is smooth. Indeed, given any $x \in M$ we can assume that the domain $A$ of the local bisection $c$ as described above is a foliated chart around $x$. Then the restriction $\left(\phi_{h}\right)_{\mid A}$ coincides with the composition $\pi_{U} \circ c$, which is smooth since both $c$ and the quotient map $\pi_{U}$ are smooth.

Therefore, $\phi_{h}$ is a smooth global bisection of $H(\mathcal{F}, \Gamma)$ such that $t \circ \phi_{h}=h$.

Now, to prove that $\Lambda\left(\phi_{h}(x)\right)=\operatorname{Hol}(h)_{x}$, let us consider a coordinate chart $U\left(\phi_{h}(x)\right)$ around $\phi_{h}(x)$ as those constructed in Proposition 1.4.2 such that $A=$ $s\left(U\left(\phi_{h}(x)\right)\right)$ is a foliated chart around $x$ and $B=t\left(U\left(\phi_{h}(x)\right)\right)$ a foliated chart around $h(x)$. Thus $\left(U\left(\phi_{h}(x)\right), t, s\right)$ can be viewed as a bi-submersion adapted 
to the twisted path holonomy atlas with quotient map given by the inclusion. Hence, up to reducing further the foliated charts, we know that there exists a bisection $\alpha: A \rightarrow U\left(\phi_{h}(x)\right)$ such that $\alpha(x)=\phi_{h}(x)$ and the diffeomorphism associated to it is $h_{\mid A}$. Choosing, now, two connected transversals $S_{x} \subseteq A$ at $x$ and $S_{y} \subseteq B$ we deduce that

$$
\Lambda_{S_{x}}^{S_{y}}\left(\phi_{h}(x)\right)=\operatorname{Hol}(h)_{x}
$$

Since the choice of transversals is irrelevant, this implies the property 2 .

Finally, for all $h_{1}, h_{2} \in \Gamma$ and $x \in M$ one has

$$
\phi_{h_{1} h_{2}}(x)=\operatorname{Hol}\left(h_{1} h_{2}\right)_{x}=\operatorname{Hol}\left(h_{1}\right)_{h_{2}(x)} \operatorname{Hol}\left(h_{2}\right)_{x}=\phi_{h_{1}}\left(h_{2}(x)\right) \phi_{h_{2}}(x),
$$

from which it follws that $h \mapsto \phi_{h}$ determines a group homomorphism from $\Gamma$ to the group of bisections $\operatorname{Bis}(H(\mathcal{F}, \Gamma))$.

We can define an action of $\Gamma$ on $H(M, \mathcal{F}, \Gamma)$ by inner automorphisms through the formula

$$
F_{h}(\gamma)=\phi_{h}(t(\gamma)) \cdot \gamma \cdot \phi_{h}(s(\gamma))^{-1}
$$

for every $\gamma \in H(M, \mathcal{F}, \Gamma)$.

Combining Proposition 1.5 .2 with the action just defined on $H(\mathcal{F}, \Gamma)$, we get the following proposition, whose proof is at this point straightforward.

Proposition 1.5.8. The above $\Gamma$-action on $H(M, \mathcal{F}, \Gamma)$ extends the $\Gamma$-action on $H(\mathcal{F})$ defined in Proposition 1.5.2.

The following remarks were suggested to us by Ralf Meyer.

Remark 1.14. Consider the holonomy groupoid $H(\mathcal{F})$ with the $\Gamma$-action given by Corollary 1.5 .3 . Then one can form the crossed product groupoid

$$
\Gamma \ltimes H(\mathcal{F})=(\Gamma \times H(\mathcal{F}), M) .
$$

For full details of this construction we refer to [20] or [39].

Since $H(\mathcal{F})$ is a Lie groupoid and the action of $\Gamma$ on $H(\mathcal{F})$ is smooth, one has that $\Gamma \ltimes H(\mathcal{F})$ is also a Lie groupoid. Moreover, the groupoid $\Gamma \ltimes H(\mathcal{F})$ acts on local transversal submanifolds of $(M, \mathcal{F})$, namely any element $(g, \alpha) \in(\Gamma \ltimes$ $H(\mathcal{F}))_{x}^{y}$ determines a unique germ at $x$ of the local diffeomorphism $h_{\alpha} \cdot \operatorname{Hol}(g)$ defined on suitable local transversals $T_{x}$ and $T_{y}$.

Now, there exists a morphism of Lie groupoids

$$
\Phi: \Gamma \ltimes H(\mathcal{F}) \rightarrow H(\mathcal{F}, \Gamma)
$$

defined by mapping an element $(h, \alpha) \in \Gamma \times H(\mathcal{F})$ to the unique element $\gamma \in$ $H(\mathcal{F}, \Gamma)$ that acts transversely on $M$ as $(h, \alpha)$ does. The uniqueness of $\gamma$ follows by Theorem 1.4.5 2). This map is surjective and therefore we get an extension of Lie groupoids

$$
1 \rightarrow \operatorname{Ker}(\Phi) \rightarrow \Gamma \ltimes H(\mathcal{F}) \rightarrow H(\mathcal{F}, \Gamma) \rightarrow 1
$$


Thus, the groupoid $H(\mathcal{F}, \Gamma)$ can be viewed as a quotient subgroupoid of the groupoid $\Gamma \ltimes H(\mathcal{F})$ modulo the subgroupoid $\operatorname{Ker}(\Phi)$.

In principle one could work with the groupoid $H(\mathcal{F}, \Gamma)$ or, equivalently, with the crossed product groupoid $\Gamma \ltimes H(\mathcal{F})$.

For our purposes, however, $H(\mathcal{F}, \Gamma)$ enjoys noteworthy properties that $\Gamma \ltimes H(\mathcal{F})$ does not satisfy in general. Indeed, by Theorem 1.4.4 $H(\mathcal{F}, \Gamma)$ is a foliation groupoid, while $\Gamma \ltimes H(\mathcal{F})$ is so only under suitable assumptions for the action. Furthermore we showed in Theorem 1.4.5 that the action of $H(\mathcal{F}, \Gamma)$ on local transversal submanifolds is effective, while that of $\Gamma \ltimes H(\mathcal{F})$ may be not, if $\operatorname{Ker}(\Phi)$ is non trivial.

For these reasons in the following we will use the groupoid $H(\mathcal{F}, \Gamma)$ rather than $\Gamma \ltimes H(\mathcal{F})$.

Remark 1.15. Let $G$ and $H$ be two Lie groupoids. Recall that a Morita equivalence $(G-H)$-bimodule $P$ between $G$ and $H$ is a space $P$ endowed with commuting left $G$-action and right $H$-action that are both free and proper, and satisfying another extra compatibility conditions on the orbit spaces $G \backslash P$ and $P / H$. For a detailed definition of action of a groupoid on a space and further details and remarks one may, for instance, consult [17], 39] and [20.

Now, let $\Gamma$ be a Lie group and suppose that $G$ and $H$ are both Lie $\Gamma$-groupoids. Then they are said to be $\Gamma$-equivariantly Morita equivalent if there exists a Morita equivalence $(G-H)$-bimodule $P$, endowed with a smooth $\Gamma$-action and such that the actions

$$
\begin{array}{r}
G \times \times_{G^{(0)}} P \times_{H^{(0)}} H \rightarrow P \\
(\gamma, p, \eta) \mapsto \gamma p \eta
\end{array}
$$

satisfy

$$
h(\gamma p \eta)=h(\gamma) h(p) h(\eta) \quad \forall h \in \Gamma .
$$

Now consider a foliated $\Gamma$-manifold $(M, \mathcal{F}, \Gamma)$ and its twisted holonomy groupoid $H(\mathcal{F}, \Gamma)$. Let us endow $H(\mathcal{F}, \Gamma)$ with the $\Gamma$-action given by Equation 1.85 and with the trivial $\Gamma$-action.

Then these two Lie $\Gamma$-groupoids are $\Gamma$-equivariantly Morita equivalent. Indeed, as Morita equivalence bimodule we can take $H(\mathcal{F}, \Gamma)$ as manifold with left and right actions of $H(\mathcal{F}, \Gamma)$ given, respectively, by left and right multiplication. Moreover one can endow the manifold $H(\mathcal{F}, \Gamma)$ with the action by diffeomorphisms given by

$$
h \cdot p=\phi_{h}(t(p)) p \quad h \in \Gamma, p \in H(\mathcal{F}, \Gamma),
$$

where $\phi_{h}$ is the bisection of $H(\mathcal{F}, \Gamma)$ associated to $h$. This is indeed an action since by Theorem 1.5.7 $\phi_{h_{1} h_{2}}=\phi_{h_{1}} \phi_{h_{2}}$ for every $h_{1}, h_{2} \in \Gamma$.

With these definitions all other axioms of equivariant Morita equivalence are easily checked and the claim follows. 


\section{Chapter 2}

\section{Foliation $\mathrm{C}^{*}$-algebras, Operators and equivariant Index classes}

\section{$2.1 \quad$ Foliation $\mathrm{C}^{*}$-algebras}

The algebras associated to the holonomy groupoid of a foliated manifold $(M, \mathcal{F})$ are one of the most important concepts in Noncommutative Geometry. Loosely speaking, they represent algebras of continuous functions on the leaf space $M / \mathcal{F}$, extending the duality of the theorem of Gel'fand-Naimark. Indeed, these algebras are in general noncommutative, since the leaf space $M / \mathcal{F}$ might have a very singular topology (cf. Section 1.3). Nevertheless many invariants for $M / \mathcal{F}$, which encode geometric and dynamical properties of the foliated manifold $(M, \mathcal{F})$, can be defined with operator algebra techniques. In this chapter, we will introduce some of these invariants, namely the $\mathrm{K}$-theory of a $\mathrm{C}^{*}$-algebra and index classes.

Throughout all the groupoids are assumed to be Hausdorff.

\subsubsection{The smooth convolution algebra associated to a Lie groupoid}

In this section we describe the convolution algebra of the compactly supported smooth functions on a Lie groupoid. The construction heavily uses the structure of groupoid and it may be thought of as a generalization of the construction of the group ring associated to a discrete group. Many important invariants are related to this algebra, in particular index classes of leafwise elliptic differential operators and cyclic cocycles of the foliation. We will introduce these invariants and explain their relationship with the smooth convolution algebra in this and in next chapters of this work.

Let $G$ be a Lie groupoid over the manifold $M$ and let us denote by $\mathfrak{g}$ the Lie 
algebroid of $G$ (for basic definitions and properties see [39]). In particular, $\mathfrak{g}$ is a vector bundle on $M$ and, if $k$ is the rank of $\mathfrak{g}$, we consider the line bundle $\Omega^{\alpha}$ of leafwise densities of order $\alpha$ on $M$. The fiber $\Omega_{x}^{\alpha}$ of $\Omega^{\alpha}$ over a point $x \in M$ is the space

$$
\Omega_{x}^{\alpha}=\left\{\sigma:\left.\Lambda^{k} \mathfrak{g}_{x} \rightarrow \mathbb{C}|\sigma(\lambda v)=| \lambda\right|^{\alpha} \sigma(v),\right\}
$$

for $\lambda \in \mathbb{R}, v \in \Lambda^{k} \mathfrak{g}_{x}$. Define

$$
\Omega^{\alpha} G=s^{*} \Omega^{\alpha} \otimes t^{*} \Omega^{\alpha}
$$

This is a line bundle over $G$, whose fiber at each $\gamma \in G$ is the tensor product $\left(\Omega^{\alpha} G\right)_{\gamma}=\Omega_{s(\gamma)}^{\alpha} \otimes \Omega_{t(\gamma)}^{\alpha}$.

Consider now the vector space $C_{c}^{\infty}\left(G, \Omega^{1 / 2} G\right)$ of compactly supported smooth sections of the bundle $\Omega^{1 / 2} G$ of half-densities on $G$. Here we need to assume that the groupoid is Hausdorff. Indeed, a non-Hausdorff manifold might not have enough smooth functions with compact support.

Using the groupoid structure of $G$, one can define on $C_{c}^{\infty}\left(G, \Omega^{1 / 2} G\right)$ the structure of an algebra with involution. Let us give the details.

If $f_{1}, f_{2}$ are sections of $C_{c}^{\infty}\left(G, \Omega^{1 / 2} G\right)$, then their convolution $f_{1} * f_{2}$ is the section of $C_{c}^{\infty}\left(G, \Omega^{1 / 2} G\right)$, defined by

$$
\left(f_{1} * f_{2}\right)(g)=\int_{g_{1} \cdot g_{2}=g} f_{1}\left(g_{1}\right) f_{2}\left(g_{2}\right)
$$

The integral is well defined. Indeed, if $g: x \rightarrow y$ is fixed, then the integrand is the 1-density

$$
g_{1} \mapsto f_{1}\left(g_{1}\right) f_{2}\left(g_{1}^{-1} \cdot g\right)
$$

defined on the manifold $G^{y}=r^{-1}(y)$. Thus, a more explicit formula, for expressing the convolution, is

$$
\left(f_{1} * f_{2}\right)(g)=\int_{G^{y}} f_{1}\left(g_{1}\right) f_{2}\left(g_{1}^{-1} \cdot g\right)
$$

The fact that the convolution of two compactly supported smooth half-densities is again a well-defined compactly supported half-density is proved, for instance, in $[12]$.

An involution on $C_{c}^{\infty}\left(G, \Omega^{1 / 2} G\right)$ is defined by the following formula. If $f \in$ $C_{c}^{\infty}\left(G, \Omega^{1 / 2} G\right)$, its adjoint $f^{*}$ is defined to be the smooth section of $C_{c}^{\infty}\left(G, \Omega^{1 / 2} G\right)$, given by

$$
f^{*}(g)=\overline{f\left(g^{-1}\right)} .
$$

It is easy to check that this mapping really defines an involution.

Lemma 2.1.1. The space $C_{c}^{\infty}\left(G, \Omega^{1 / 2} G\right)$ endowed with the convolution and the involution defined above becomes a*-algebra.

Proof. It is a standard fact that the convolution operation as defined above is associative, i.e. $f_{1} *\left(f_{2} * f_{3}\right)=\left(f_{1} * f_{2}\right) * f_{3}$ and distributive. Thus it defines the structure of an associative algebra on $C_{c}^{\infty}\left(G, \Omega^{1 / 2} G\right)$. Moreover it is not difficult to verify that $\left(f_{1} * f_{2}\right)^{*}=f_{1}^{*} * f_{2}^{*}$ and $\left(f_{1}+f_{2}\right)^{*}=f_{1}^{*}+f_{2}^{*}$. 
Notice the quite clear but important fact that the algebra $C_{c}^{\infty}\left(G, \Omega^{1 / 2} G\right)$ is no longer commutative.

Remark 2.1. The use of half-densities in the definition of the convolution algebra shows how canonical the construction is. However, if one prefers to work with functions instead of densities, let us observe that any positive smooth halfdensity on $M$ induces a trivialization of $\Omega^{1 / 2} G$. Using this trivialization, one may define the convolution between two smooth compactly supported functions with respect to the chosen density by means of the same formula.

Remark 2.2. If $G$ is not Hausdorff, it is still possible to construct a canonical $*$-algebra associated to the groupoid. The construction is more involved and use in an essential way the local structure of $G$, which is very simple, being $G$ locally diffeomorphic to an Euclidean space.

The details of this construction can be found in [12] or in 14 .

Now, let $(M, \mathcal{F})$ be a foliated manifold and let $H(M, \mathcal{F})$ be its holonomy groupoid. We have seen in the previous chapter that $H(M, \mathcal{F})$ is a Lie groupoid (Proposition 1.3.6), in general not Hausdorff. Let us restrict our attention on foliated manifolds with Hausdorff holonomy groupoid (this is a non trivial hypothesis. See 12 for examples of foliations, whose holonomy groupoid is not Hausdorff). Analogously, we consider $\Gamma$-foliated manifolds $(M, \mathcal{F}, \Gamma)$ whose associated twisted holonomy groupoid $H(\mathcal{F}, \Gamma)$, is Hausdorff.

Definition 2.1. $\quad$ 1. To any foliated manifold $(M, \mathcal{F})$ we associate the $*-$ algebra $C_{c}^{\infty}\left(H(\mathcal{F}), \Omega^{1 / 2} H(\mathcal{F})\right)$ of the holonomy groupoid $H(\mathcal{F})$ of the foliation. We shall call it the smooth convolution algebra of the foliated manifold.

2. Similarly, if $(M, \mathcal{F}, \Gamma)$ is a $\Gamma$-foliated manifold, the involutive algebra $C_{c}^{\infty}\left(H(\mathcal{F}, \Gamma), \Omega^{1 / 2} H(\mathcal{F}, \Gamma)\right)$ of the twisted holonomy groupoid will be called the smooth convolution algebra of the $\Gamma$-foliated manifold.

These algebras play an important role in the study of foliated manifolds by means of methods coming from Noncommutative Geometry. Indeed, we shall see that many invariants of a foliation are naturally defined on them.

\subsubsection{The reduced $\mathrm{C}^{*}$-algebras associated to a Lie groupoid}

In order to obtain the $C^{*}$-algebras associated with a Lie groupoid and, in particular, the foliation $C^{*}$-algebras canonically related to a foliated manifold $(M, \mathcal{F})$, the idea is to complete the involutive algebra $C_{c}^{\infty}\left(G, \Omega^{1 / 2} G\right)$ with respect to various norms. The norms on $C_{c}^{\infty}\left(G, \Omega^{1 / 2} G\right)$ are induced by suitable representations into the $C^{*}$-algebra of bounded operators on a Hilbert space. Thus, the $C^{*}$-algebras of a Lie groupoid are closed involutive subalgebras of bounded operators with respect to the operator norm.

Let $G$ be a Lie groupoid over the manifold $M$ and $\mathfrak{g}$ its Lie algebroid of rank $k$. For each point $x \in M$ there is a natural representation $R_{x}$ of $C_{c}^{\infty}\left(G, \Omega^{1 / 2} G\right)$ on the Hilbert space of half-densities on the $s$-fibers $G_{x}$ of the groupoid. 
Indeed, let $\Omega^{1 / 2} G_{x}$ denote the vector bundle of $1 / 2$-densities on the submanifold $G_{x}=s^{-1}(x)$ and $L^{2}\left(G_{x}, \Omega^{1 / 2} G_{x}\right)$ the Hilbert space of square integrable densities.

Recall, now, that there exists a canonical bundle isomorphism $\operatorname{ker}(d s) \cong t^{*}(\mathfrak{g})$. Then, if $\Omega^{1 / 2}$ is the line bundle of leafwise densities of order $1 / 2$ on $M$, as defined in the previous section, one has the canonical identification $\Omega^{1 / 2} G_{x} \cong t^{*} \Omega^{1 / 2}$. Thus, for any $\gamma \in G_{x}$, the fiber $\left(\Omega^{1 / 2} G_{x}\right)_{\gamma} \cong\left(\Omega^{1 / 2}\right)_{t(\gamma)}$.

For any point $x \in M$, and any $f \in C_{c}^{\infty}\left(G, \Omega^{1 / 2} G\right)$, consider the operator

$$
R_{x}(f): L^{2}\left(G_{x}, \Omega^{1 / 2} G_{x}\right) \rightarrow L^{2}\left(G_{x}, \Omega^{1 / 2} G_{x}\right)
$$

defined by

$$
\left(R_{x}(f) \xi\right)(\gamma)=\int_{\gamma_{1} \cdot \gamma_{2}=\gamma} f\left(\gamma_{1}\right) \xi\left(\gamma_{2}\right)
$$

This definition is well-posed. Indeed, first observe that, for fixed $\gamma: x \rightarrow y$, the density $\xi\left(\gamma_{2}\right) \in\left(\Omega^{1 / 2} G_{x}\right)_{\gamma_{2}} \cong\left(\Omega^{1 / 2}\right)_{t\left(\gamma_{2}\right)}$ and $f\left(\gamma_{1}\right) \in\left(\Omega^{1 / 2} G\right)_{\gamma_{1}} \cong\left(\Omega^{1 / 2}\right)_{t\left(\gamma_{1}\right)} \otimes$ $\left(\Omega^{1 / 2}\right)_{s\left(\gamma_{1}\right)}$. Since $t\left(\gamma_{2}\right)=s\left(\gamma_{1}\right), f\left(\gamma_{1}\right) \xi\left(\gamma_{2}\right)$ is a 1 -density on the manifold $\left\{\gamma_{1} \cdot \gamma_{2}=\gamma\right\}$ with values in $\left(\Omega^{1 / 2}\right)_{t(\gamma)=t\left(\gamma_{1}\right)} \cong\left(\Omega^{1 / 2} G_{x}\right)_{\gamma}$.

The integral $\left(R_{x}(f) \xi\right)(\gamma)$ is, thus, a well-defined element of $\left(\Omega^{1 / 2} G_{x}\right)_{\gamma}$. Setting $\gamma_{1}=\gamma \cdot \gamma_{2}^{-1}$, it is clear that $\left\{\gamma_{1} \cdot \gamma_{2}=\gamma\right\}=G_{x}$ and we can rewrite

$$
\left(R_{x}(f) \xi\right)(\gamma)=\int_{G_{x}} f\left(\gamma \cdot \gamma_{2}^{-1}\right) \xi\left(\gamma_{2}\right)
$$

The above expression 2.8 shows that $R_{x}(f)$ is an integral operator on $L^{2}\left(G_{x}, \Omega^{1 / 2} G_{x}\right)$ with smooth compactly supported kernel $F\left(\gamma_{1}, \gamma_{2}\right)=f\left(\gamma_{1} \cdot \gamma_{2}^{-1}\right)$. Thus, in particular, it defines a bounded operator. For a proof of this statement, also valid in the non-Hausdorff case, we recommend 12 .

Lemma 2.1.2. For each $x \in M$, the map

$$
R_{x}: C_{c}^{\infty}\left(G, \Omega^{1 / 2} G\right) \rightarrow \mathcal{B}\left(L^{2}\left(G_{x}, \Omega^{1 / 2} G_{x}\right)\right)
$$

is an involutive representation of the $*$-algebra $C_{c}^{\infty}\left(G, \Omega^{1 / 2} G\right)$.

Proof. By definition, we have to prove that $R_{x}\left(f_{1} * f_{2}\right)=R_{x}\left(f_{1}\right) \circ R_{x}\left(f_{2}\right)$ and that $R_{x}\left(f^{*}\right)=\left(R_{x}(f)\right)^{*}$. The first equality follows from the associativity of the multiplication in $G$. For the second one, recall that the Schwartz kernel of the bounded operator $R_{x}(f)$ is $F\left(\gamma_{1}, \gamma_{2}\right)=f\left(\gamma_{1} \cdot \gamma_{2}^{-1}\right)$ and that the Schwartz kernel of the adjoint $\left(R_{x}(f)\right)^{*}$ is given by $\overline{F\left(\gamma_{1}, \gamma_{2}\right)}=\overline{f\left(\gamma_{1} \cdot \gamma_{2}^{-1}\right)}$. This proves the lemma.

The family of representations $\left\{R_{x}\right\}_{x \in M}$ defined above is called the family of right regular representations of $C_{c}^{\infty}\left(G, \Omega^{1 / 2} G\right)$.

Given any element $\gamma \in G, \gamma: x \rightarrow y$, one has a natural unitary operator $U_{\gamma}: L^{2}\left(G_{x}, \Omega^{1 / 2} G_{x}\right) \rightarrow L^{2}\left(G_{y}, \Omega^{1 / 2} G_{y}\right)$ given by

$$
\left(U_{\gamma}(\xi)\right)\left(\gamma_{1}\right)=\xi\left(\gamma_{1} \cdot \gamma\right)
$$

One gets the following result, whose proof is straightforward. 
Lemma 2.1.3. Given any $f \in C_{c}^{\infty}\left(G, \Omega^{1 / 2} G\right)$ and any element $\gamma \in G$, the operator $U_{\gamma}$ intertwines the operators $R_{s(\gamma)}(f)$ and $R_{t(\gamma)}(f)$, precisely $R_{s(\gamma)} U_{\gamma}=$ $U_{\gamma} R_{t(\gamma)}$.

Roughly speaking, the above lemma states that the family $\left\{R_{x}\right\}$ induces a family of representations $\left\{R_{L}\right\}_{L \in M / \mathcal{F}}$ parametrized by the space of leaves of the foliated manifold $(M, \mathcal{F})$. Indeed, if $x, y \in M$ are in the same leaf $L$, then the representations $R_{x}$ and $R_{y}$ are equivalent. In particular, the norm $\left\|R_{x}(f)\right\|$ only depends on the leaf $L_{x}$ through $x$.

We are, now, ready to give the following fundamental definitions.

Definition 2.2. $\quad$ 1. The reduced $C^{*}$-algebra of the Lie groupoid $G$ over $M$ is the completion of the involutive algebra $C_{c}^{\infty}\left(G, \Omega^{1 / 2} G\right)$ with respect to the norm

$$
\|f\|=\sup _{x \in M}\left\|R_{x}(f)\right\|
$$

This algebra is denoted by $C_{r}^{*}(G)$.

2. Let $(M, \mathcal{F})$ be a foliated manifold and $H(\mathcal{F})$ its holonomy groupoid. The reduced $C^{*}$-algebra of the foliated manifold is the reduced $C^{*}$-algebra of the holonomy groupoid $H(\mathcal{F})$. This $C^{*}$-algebra is denoted by $C_{r}^{*}(M, \mathcal{F})$.

3. If $(M, \mathcal{F}, \Gamma)$ is a foliated $\Gamma$-manifold and $H(\mathcal{F}, \Gamma)$ is its twisted holonomy groupoid, then the reduced $C^{*}$-algebra of the foliated $\Gamma$-manifold is the reduced $C^{*}$-algebra of the twisted holonomy groupoid $H(\mathcal{F}, \Gamma)$. We will denote it by $C_{r}^{*}(M, \mathcal{F}, \Gamma)$.

It is not difficult to verify that the norm

$$
\|f\|=\sup _{x \in M}\left\|R_{x}(f)\right\|
$$

is indeed a $C^{*}$-norm.

Lemma 2.1.4. If $f \in C_{c}^{\infty}\left(G, \Omega^{1 / 2} G\right)$ is not identically zero, then there is $x \in M$ such that $R_{x}(f) \neq 0$.

Proof. Let $f \in C_{c}^{\infty}\left(G, \Omega^{1 / 2} G\right)$ and $\gamma \in G$ with $f(\gamma) \neq 0$. Then, there exists a coordinate neighborhood $U(\gamma)$ of $\gamma$ on which $f$ does not vanish. Let $x=s(\gamma)$ and consider the neighborhood $V=U(\gamma) \cap G_{x}$ of $\gamma$ in $G_{x}$. Then, of course, $f$ does not vanish on $V$. Choose, now, a smooth positive function $\xi$ with compact support contained in an open neighborhood of $1_{x}$ in $G_{x}$. Then $R_{x}(f)(\xi)$ is evidently nonzero and therefore the operator $R_{x}(f)$ is nontrivial. Hence $\|f\| \neq$ 0 .

The definition of the regular representations implies, of course, that for each $x \in M$

$$
R_{x}: C_{c}^{\infty}\left(G, \Omega^{1 / 2} G\right) \rightarrow \mathcal{B}\left(L^{2}\left(G_{x}, \Omega^{1 / 2} G_{x}\right)\right)
$$

is continuous with respect to this norm. Therefore $R_{x}$ extends by continuity to a representation of $C_{r}^{*}(G)$. 
Important Remark 2.1. If $E$ is a Hermitian vector bundle over the space of objects $G^{(0)}=M$ of the Lie groupoid $G$, then one can introduce the reduced $C^{*}$-algebra with coefficients in $E$. Precisely, one defines the bundle $E N D(E)=$ $\left(s^{*}(E)\right)^{*} \otimes t^{*}(E)$ on $G$ and takes the space $C_{c}^{\infty}\left(G, E N D(E) \otimes \Omega^{1 / 2} G\right)$ of compactly supported smooth sections of the tensor product $E N D(E) \otimes \Omega^{1 / 2} G$. On this space one can define a convolution and an involution exactly as in the scalar case. Now, letting $C_{c}^{\infty}\left(G, E N D(E) \otimes \Omega^{1 / 2} G\right)$ act on the fields of Hilbert spaces $\left.L_{\tau}^{2}\left(G, t^{*}(E)\right)=\left\{L^{2}\left(G_{x}, t^{*}(E) \otimes \Omega^{1 / 2} G_{x}\right)\right)\right\}_{x \in M}$ as in 2.8 , we get a family of representations denoted again by $\left\{R_{x}\right\}_{x \in M}$.

One completes, now, $C_{c}^{\infty}\left(G, E N D(E) \otimes \Omega^{1 / 2} G\right)$ with respect to these representations, obtaining the reduced $C^{*}$-algebra with coefficients denoted by $C_{r}^{*}(G, E)$. It can be proved that $C_{r}^{*}(G)$ and $C_{r}^{*}(G, E)$ are Morita equivalent (see [40, pag. 141 for a proof).

The reduced $C^{*}$-algebra $C_{r}^{*}(G)$ of a Lie groupoid $G$ reflects many features and properties of the groupoid itself. In the case of a foliated manifold $(M, \mathcal{F})$ or of a $\Gamma$-foliated manifold $(M, \mathcal{F}, \Gamma)$, the algebras $C_{r}^{*}(M, \mathcal{F})$ and $C_{r}^{*}(M, \mathcal{F}, \Gamma)$ encode geometric and dynamical features of the foliation, in particular related to the transverse geometry of the foliation.

In this regard we state below two theorems, suggesting the deep interplay between the geometry of a foliated manifold and the representations of the foliation $C^{*}$-algebra.

Besides, we shall see many other remarkable aspects and consequences of this interaction in the coming sections of this thesis.

Theorem 2.1.5. Let $(M, \mathcal{F})$ be a foliated manifold. Then the representation $R_{x}$ of $C_{r}^{*}(M, \mathcal{F})$ is irreducible if and only if the leaf $L_{x}$ through $x$ has trivial holonomy.

Theorem 2.1.6. (Fack-Skandalis) Let $(M, \mathcal{F})$ be a foliated manifold. Then the foliation $(M, \mathcal{F})$ is minimal, i.e. each leaf $L$ of $\mathcal{F}$ is dense in $M$, if and only if the foliation $C^{*}$-algebra $C_{r}^{*}(M, \mathcal{F})$ is simple.

For a detailed proof we refer to [12.

The construction of the foliation $C^{*}$-algebra $C_{r}^{*}(M, \mathcal{F})$ is local in the following sense.

Proposition 2.1.7. ([12]) Let $(M, \mathcal{F})$ be a foliated manifold and let $\mathcal{U}=$ $\left\{\left(U_{i}, x_{i}, y_{i}\right)\right\}_{i \in I}$ a regular foliated atlas. Then the algebra generated by the convolution algebras $C_{c}^{\infty}\left(H(U, \mathcal{F}), \Omega^{1 / 2} H(U, \mathcal{F})\right)$, with $U \in \mathcal{U}$, is dense in $C_{r}^{*}(M, \mathcal{F})$.

In the proposition, the symbol $H(U, \mathcal{F})$ denotes the open subgroupoids of $H(\mathcal{F})$ given by the $s$-connected component of the restrictions $H(\mathcal{F})_{U}^{U}=$ $t^{-1}(U) \cap s^{-1}(U)$ of the holonomy groupoid at $U$.

We will see in a moment that the structure of the $C^{*}$-algebras $C_{r}^{*}(H(U, \mathcal{F}))$ is rather elementary, reflecting the local triviality of the foliation and being essentially continuous functions on the transversal manifold $T \subseteq U$ vanishing at infinity. At the other side, the structure of $C_{r}^{*}(M, \mathcal{F})$ can be very complicated, 
depending on the intricate way, in which the foliation charts $U$ assemble to give the foliation.

Proposition 2.1.8. Let $(M, \mathcal{F})$ be a foliated manifold and let $V \subseteq M$ be an open set, foliated by the restriction $\mathcal{F}_{V}$ of $\mathcal{F}$ to $V$. Then the holonomy groupoid $H\left(V, \mathcal{F}_{V}\right)$ of $\left(V, \mathcal{F}_{V}\right)$ is an open subgroupoid of the holonomy groupoid $H(M, \mathcal{F})$ of $(M, \mathcal{F})$. Moreover, the inclusion $H\left(V, \mathcal{F}_{V}\right) \subseteq H(M, \mathcal{F})$ induces an isometric *-homomorphism of $C_{r}^{*}\left(V, \mathcal{F}_{V}\right)$ into $C_{r}^{*}(M, \mathcal{F})$.

Proof. We refer to [12, Proposition 1.5.5 for a proof of this result.

Let $(M, \mathcal{F}, \Gamma)$ be a $\Gamma$-foliated manifold. We have seen in Lemma 1.4.1 and in Proposition 1.4 .3 that the natural inclusion map

$$
j: H(\mathcal{F}) \hookrightarrow H(\mathcal{F}, \Gamma)
$$

is a morphism of Lie groupoids, i.e a smooth functor between Lie groupoids, and also a local diffeomorphism. Thus, we deduced that the holonomy groupoid is an open $s$-connected Lie subgroupoid over $M$ of the twisted holonomy groupoid $H(\mathcal{F}, \Gamma)$.

Furthermore, one has the induced involutive algebra homomorphism

$$
j: C_{c}^{\infty}\left(H(\mathcal{F}), \Omega^{1 / 2} H(\mathcal{F})\right) \rightarrow C_{c}^{\infty}\left(H(\mathcal{F}, \Gamma), \Omega^{1 / 2} H(\mathcal{F}, \Gamma)\right)
$$

defined by "extension by 0" (since the groupoids are Hausdorff, this is well defined).

Let us, now, mention some very basic examples of foliation $C^{*}$-algebras for particular classes of foliated manifolds, which can be explicitly computed.

Example 2.1. Let us consider first a trivial foliated manifold $M=U \times T$, with $U$ a connected manifold. In this case there is no non trivial holonomy and thus the holonomy groupoid $H$ of $U \times T$ can be identified with $U \times U \times T$, with source map $s(x, y, t)=(y, t)$ and target map $t(x, y, t)=(x, t)$. The product takes the form

$$
(x, y, t) \cdot\left(x^{\prime}, y^{\prime}, t^{\prime}\right)=\left(x, y^{\prime}, t\right)
$$

if $t=t^{\prime}$ and $x^{\prime}=y$.

Proposition 2.1.9. ([12] ) The reduced $C^{*}$-algebra $C_{r}^{*}(U \times T)$ of the trivial foliated manifold $U \times T$ is isomorphic to the tensor product $\mathcal{K} \otimes C_{0}(T)$, where $C_{0}(T)$ denotes the continuous functions on the transversal $T$ vanishing at infinity and $\mathcal{K}$ is the elementary $C^{*}$-algebra of compact operators on $L^{2}(U)$.

Then, up to stabilizing with compact operators, the foliation $C^{*}$-algebra is the same as the commutative $C^{*}$-algebra of continuous functions defined on the manifold $T$ transversal to the leaves.

Let $(M, \mathcal{F})$ be an arbitrary foliated manifold and let $U \subseteq M$ be a foliated chart of $M$. Then $\left(U, \mathcal{F}_{U}\right)$ is a trivial foliated space and, by Proposition 2.1.8, one gets the isometric $*$-homomorphism $i: C_{r}^{*}\left(U, \mathcal{F}_{U}\right) \rightarrow C_{r}^{*}(M, \mathcal{F})$. Moreover, as 
a consequence of Proposition 2.1.7 we also know that $C_{r}^{*}(M, \mathcal{F})$ is generated by the $C^{*}$-subalgebras $C_{r}^{*}\left(U, \mathcal{F}_{U}\right)$, which essentially are continuous functions on the local transversal.

Heuristically, this means that the foliation $C^{*}$-algebra $C_{r}^{*}(M, \mathcal{F})$ only depends upon the transverse geometry of the foliation and may be thought of as the algebra of continuous functions on the space of leaves.

Example 2.2. Let us consider the geometric setup described in Example 1.1. In other words, let us assume to have a foliated manifold $(M, \mathcal{F})$, whose leaves are the fibers of a fibre bundle projection $\pi: M \rightarrow B$ (we assume the tipical fiber $F$ of the fiber bundle to be a connected manifold). Recall that such a foliation is called simple.

In this case, one has the following result.

Proposition 2.1.10. The reduced $C^{*}$-algebra of the simple foliation $(M, \mathcal{F})$ is isomorphic to $C_{0}(B) \otimes \mathcal{K}\left(L^{2}(F)\right)$.

Thus, also in this example as in the previous one, we may heuristically think of the foliation $C^{*}$-algebra of a simple foliation $(M, \mathcal{F})$ as being the algebra of continuous functions on the space of leaves of the foliation.

\subsubsection{Automorphisms defined by a group $\Gamma$}

After having seen how to associate various algebras to any Lie groupoid, we will study in this section their behaviour, if the Lie groupoid $G$ is further a Lie $\Gamma$-groupoid. In this case it is not surprising that the convolution algebras inherite also an action of the group $\Gamma$ by algebra automorphisms. More surprising, however, is the fact that, if the action of $\Gamma$ on the Lie groupoid $G$ is by inner automorphisms, then each element of $\Gamma$ gives rise to a multiplier of the groupoid $C^{*}$-algebra, which preserves the smooth convolution algebra. This will be important for constructing equivariant cyclic cocycles on the groupoid. Finally, we will specialize to the case of a foliated $\Gamma$-manifold, applying the results obtained for general Lie $\Gamma$-groupoid to the holonomy groupoid and the twisted holonomy groupoid of the foliation.

Many results of this section are generalizations to general Lie $\Gamma$-groupoids of results proven in [7] for the holonomy groupoid.

Thus, let $\mathcal{A}$ be a not necessarily unital $\mathbb{C}$-algebra and let $\Gamma$ denote a Lie group.

Definition 2.3. $\quad$. The algebra $\mathcal{A}$ is called a $\Gamma$-algebra if there is a group homomorphism

$$
\rho: \Gamma \rightarrow \operatorname{Aut}(\mathcal{A})
$$

where $\operatorname{Aut}(\mathcal{A})$ denotes the group of the algebra automorphisms of $\mathcal{A}$.

Equivalently, we will call the triple $(\mathcal{A}, \Gamma, \rho)$ a $\Gamma$-algebra or also noncommutative dynamical system.

If $\mathcal{A}$ is endowed with an involution $*$, we further require that

$$
\rho_{h}\left(a^{*}\right)=\rho_{h}(a)^{*}
$$


for every $h \in \Gamma, a \in \mathcal{A}$.

In this case, $\mathcal{A}$ is said to be a $\Gamma-*-$ algebra.

2. If the algebra $\mathcal{A}$ is a topological algebra, we will call $\mathcal{A}$ a $\Gamma$-algebra, if there is a group homomorphism $\rho: \Gamma \rightarrow \operatorname{Aut}(\mathcal{A})$, in the continuous automorphisms which is strongly continuous, i.e. for every $a \in \mathcal{A}$ the map

$$
g \mapsto \rho_{g}(a) \in \mathcal{A}
$$

is continuous with respect to the topology on $\mathcal{A}$.

We will often forget to mention explicitly the homomorphism $\rho$ if there is no cause of confusion.

Examples of $\Gamma$-algebras arise naturally in Mathematics. The following is, perhaps, the simplest one.

Example 2.3. Let $M$ be a closed smooth $\Gamma$-manifold, with $\Gamma$ a compact Lie group acting smoothly (on the left) on $M$ by diffeomorphisms. Then the algebra of smooth functions $C^{\infty}(M)$ inherits a natural $\Gamma$-action, given by $\rho_{g}(f)(p):=$ $f\left(g^{-1}(p)\right)$ and this action is naturally strongly continuous with respect to the Frèchet topology of $C^{\infty}(M)$, since the action $\Gamma$ on $M$ is smooth.

Thus, the Fréchet algebra $C^{\infty}(M)$ endowed with the action $\rho$ is a $\Gamma$-algebra.

In this section we will see that, for any Lie $\Gamma$-groupoid $G$, the smooth convolution algebra $C_{c}^{\infty}\left(G, \Omega^{1 / 2} G\right)$ and the $C^{*}$-algebra $C_{r}^{*}(G)$ are $\Gamma$-algebras, as well as the algebras $C_{c}^{\infty}\left(G, E N D(E) \otimes \Omega^{1 / 2} G\right)$ and $C_{r}^{*}(G, E)$ with values in the equivariant vector bundle $E$ over $M$ (see Remark 2.1 for details).

Thus, let now $G$ be a Lie $\Gamma$-groupoid (see Def. 1.29 ) and let $E$ be a $\Gamma$-equivariant vector bundle over its space of objects $G^{0}=M$. We endow the bundle $E$ with a Hermitian metric which is preserved by $\Gamma$. This is always possible, if the group $\Gamma$ is compact.

Consider the smooth convolution algebra $C_{c}^{\infty}\left(G, E N D(E) \otimes \Omega^{1 / 2} G\right)$ of smooth half densities over $G$ with values in $E N D(E)=\operatorname{Hom}\left(s^{*}(E), t^{*}(E)\right)=s^{*}\left(E^{*}\right) \otimes$ $t^{*}(E)$ (see Remark 2.1).

On the bundles $\Omega^{1 / 2} G$ and $E N D(E)$ one can canonically induce actions of $\Gamma$ from the given $\Gamma$-action on $G$ and $E$. Indeed, the Lie algebroid $\mathfrak{g}$ of $G$ inherits a natural $\Gamma$-action by Lie algebroid morphisms (for details on this point, see [38] which in turn induces an action of $\Gamma$ on the bundle $\Omega^{1 / 2}$ of transversal densities on $M$. This turns $\Omega^{1 / 2}$ into a $\Gamma$-equivariant vector bundle.

Further notice that for any $\Gamma$-equivariant bundle $E$ over $M$, the pull-back bundle $s^{*}(E)$, with respect to the source map, inherits a natural action of $\Gamma$, that makes it into a $\Gamma$-equivariant bundle on $G$. In the same way, the pull-back $t^{*}(E)$, with respect to the target map, becomes a $\Gamma$-equivariant bundle over $G$. Hence, the bundle $\Omega^{1 / 2} G \otimes E N D(E)=s^{*}\left(\Omega^{1 / 2}\right) \otimes t^{*}\left(\Omega^{1 / 2}\right) \otimes H o m\left(s^{*}(E), t^{*}(E)\right)$ is naturally a $\Gamma$-equivariant bundle over $G$ with the diagonal action.

Important Remark 2.2. To distinguish the various actions, we will denote the action of an element $h$ on $\Omega^{1 / 2} \otimes E$ by $A_{h}$, and that on $\Omega^{1 / 2} \otimes E^{*}$ by $A_{h}^{*}$. The 
induced action on the bundle $\Omega^{1 / 2} G \otimes E N D(E)$ over $G$ will be simply denoted by $h$.

Since $\Omega^{1 / 2} G \otimes E N D(E)=t^{*}\left(\Omega^{1 / 2} \otimes E\right) \otimes s^{*}\left(\Omega^{1 / 2} \otimes E^{*}\right)$, one has for $T \in$ $\left(\Omega^{1 / 2} G \otimes E N D(E)\right)_{\gamma}$ that $h \cdot T=\left(A_{h} \otimes A_{h}^{*}\right) \cdot T \in\left(\Omega^{1 / 2} G \otimes E N D(E)\right)_{h(\gamma)}$.

The latter notation has the advantage to make clear that we are acting simultaneously on the "source-part" and on "target-part" of the section $T$. If we want to denote only the action on the "source-part", we will write $i d \otimes A_{h}^{*}$. Similarly, we will write $A_{h} \otimes i d$ to indicate the action of $h$ only on the "target-part".

Finally, observe that the action on $E N D(E)$ is given by conjugation with the action on the bundle $E$. Then, with a small abuse of notation given by mentioning implicitly the action on half-densities, we will write $\left(A_{h} \otimes A_{h}^{*}\right) \cdot T=$ $A_{h} T A_{h}^{-1} \in\left(\Omega^{1 / 2} G \otimes E N D(E)\right)_{h(\gamma)}$.

Using both actions on $G$ and on $\Omega^{1 / 2} G \otimes E N D(E)$, we set

$$
(h \cdot f)(\gamma)=h \cdot f\left(h^{-1}(\gamma)\right) \quad \forall \gamma \in G
$$

for any $h \in \Gamma$ and $f \in C_{c}^{\infty}\left(G, E N D(E) \otimes \Omega^{1 / 2} G\right)$.

Lemma 2.1.11. Equation 2.13 defines a $\Gamma$-action on $C_{c}^{\infty}\left(G, E N D(E) \otimes \Omega^{1 / 2} G\right)$, which turns it into a $\Gamma-*$-algebra.

Proof. The proof follows directly by applying the definition of convolution and involution on $C_{c}^{\infty}\left(G, E N D(E) \otimes \Omega^{1 / 2} G\right)$.

Consider, now, the field of Hilbert spaces $L_{\tau}^{2}\left(G, t^{*}(E)\right)=\left\{L^{2}\left(G_{x}, t^{*}(E) \otimes\right.\right.$ $\left.\left.\left.\Omega^{1 / 2} G_{x}\right)\right)\right\}_{x \in M}$ over $M$.

For each element $h \in \Gamma$, we define the operator

$$
\begin{aligned}
U_{h}: L^{2}\left(G_{x}, \Omega^{1 / 2} G_{x} \otimes t^{*}(E)\right) & \rightarrow L^{2}\left(G_{h(x)}, \Omega^{1 / 2} G_{h(x)} \otimes t^{*}(E)\right) \\
\xi & \mapsto \quad\left(U_{h} \xi\right)(\gamma)=A_{h} \cdot \xi\left(h^{-1}(\gamma)\right) .
\end{aligned}
$$

Since the Hermitian metric on $E$ has been chosen to be $\Gamma$-invariant, the operator $U_{h}$ defines a unitary operator between Hilbert spaces for any point $x \in M$. Recall that the algebra $C_{c}^{\infty}\left(G, E N D(E) \otimes \Omega^{1 / 2} G\right)$ acts on $L_{\tau}^{2}\left(G, t^{*}(E)\right)$ through the right regular representation $R=\left\{R_{x}\right\}_{x \in M}$ as given by Equation 2.8. One has the following lemma.

Lemma 2.1.12. The pair $(R, U)$ defines a covariant representation of the pair $\left(C_{c}^{\infty}\left(G, E N D(E) \otimes \Omega^{1 / 2} G\right), \Gamma\right)$ on the field of Hilbert spaces $L_{\tau}^{2}\left(G, t^{*}(E)\right)$, i.e. it holds that

$$
R_{x}(h \cdot k)=U_{h} \circ R_{h^{-1}(x)}(k) \circ U_{h^{-1}}
$$

for all $h \in \Gamma, k \in C_{c}^{\infty}\left(G, E N D(E) \otimes \Omega^{1 / 2} G\right), x \in M$.

Proof. Let us briefly sketch the proof. Let $\left.\xi \in L^{2}\left(G_{x}, t^{*}(E) \otimes \Omega^{1 / 2} G_{x}\right)\right)$ and $\gamma \in G_{x}$. Then

$$
\left(R_{x}(h \cdot k)(\xi)\right)(\gamma)=\int_{G_{x}}(h \cdot k)\left(\gamma \gamma_{1}^{-1}\right) \xi\left(\gamma_{1}\right)=
$$




$$
\begin{gathered}
=\int_{G_{x}}\left[h \cdot k\left(h^{-1}(\gamma) h^{-1}\left(\gamma_{1}^{-1}\right)\right)\right] \xi\left(\gamma_{1}\right)=\int_{G_{x}}\left[\left(A_{h} \otimes A_{h}^{*}\right) \cdot k\left(h^{-1}(\gamma) h^{-1}\left(\gamma_{1}^{-1}\right)\right)\right] \xi\left(\gamma_{1}\right)= \\
=\int_{G_{x}} A_{h} \cdot k\left(h^{-1}(\gamma) h^{-1}\left(\gamma_{1}^{-1}\right)\right) A_{h}^{-1} \cdot \xi\left(\gamma_{1}\right) .
\end{gathered}
$$

Now, using the invariance of the integral of densities with respect to diffeomorphisms and setting $\eta=h^{-1}\left(\gamma_{1}\right)$, one gets that

$$
\begin{gathered}
\int_{G_{x}} A_{h} \cdot k\left(h^{-1}(\gamma) h^{-1}\left(\gamma_{1}^{-1}\right)\right) A_{h}^{-1} \cdot \xi\left(\gamma_{1}\right)=\int_{G_{h^{-1}(x)}} A_{h} \cdot k\left(h^{-1}(\gamma) \eta^{-1}\right) A_{h}^{-1} \cdot \xi(h(\eta))= \\
=\int_{G_{h^{-1}(x)}} A_{h} \cdot k\left(h^{-1}(\gamma) \eta^{-1}\right)\left(U_{h^{-1}} \xi\right)(\eta)=A_{h} \cdot \int_{G_{h^{-1}(x)}} k\left(h^{-1}(\gamma) \eta^{-1}\right)\left(U_{h^{-1}} \xi\right)(\eta)= \\
=\left(U_{h} \circ R_{h^{-1}(x)}(k) \circ U_{h^{-1}}\right)(\xi)(\gamma) .
\end{gathered}
$$

By the Lemma above, it is now evident that each $h \in \Gamma$ gives rise to an automorphism $T_{h}=U_{h} \cdot U_{h^{-1}}$ of $C_{c}^{\infty}\left(G, E N D(E) \otimes \Omega^{1 / 2} G\right)$, that is bounded with respect to the norm 2.10

Lemma 2.1.13. For every $h \in \Gamma$ the automorphism $T_{h}=U_{h} \cdot U_{h^{-1}}$ extends to $a *$-automorphism of $C_{r}^{*}(G, E)$ giving rise to an action of $\Gamma$ on $C_{r}^{*}(G, E)$. Moreover, this action is strongly continuous.

Proof. Since $C_{c}^{\infty}\left(G, E N D(E) \otimes \Omega^{1 / 2} G\right) \subseteq C_{r}^{*}(G, E)$ is dense and $T_{h}$ is bounded, it follows that each $T_{h}$ extends by continuity to an automorphism of $C_{r}^{*}(G, E)$. In this way, we get an induced action on the $C^{*}$-algebra. The fact that this action is also strongly continuous follows from the continuity of all the actions on the groupoid and on the bundles.

Summarizing, the reduced $C^{*}$-algebra $C_{r}^{*}(G, E)$ of any Lie $\Gamma$-groupoid $G$ with values in a $\Gamma$-equivariant vector bundle $E$, endowed with a $\Gamma$-invariant Hermitian structure, is a $\Gamma-C^{*}$-algebra. Moreover, this action preserves the smooth subalgebra $C_{c}^{\infty}\left(G, E N D(E) \otimes \Omega^{1 / 2} G\right)$.

For our purposes the case where the $\Gamma$-action on the Lie groupoid is given by inner automorphisms deserves particular attention.

Let us assume therefore to have a group homomorphism $\sigma: \Gamma \rightarrow B i s(G)$ inducing the action on $G$. Recall that $\operatorname{Bis}(G)$ is the group of smooth global bisections of $G$ and that such a homomorphism defines an action by inner automorphisms (see Section 1.5 of Chapter 1).

If $E$ is an equivariant vector bundle with a $\Gamma$-invariant metric, for each $h \in \Gamma$ we can define the unitary operator

$$
S(h)_{x}: L^{2}\left(G_{x}, \Omega^{1 / 2} G_{x} \otimes t^{*}(E)\right) \rightarrow L^{2}\left(G_{x}, \Omega^{1 / 2} G_{x} \otimes t^{*}(E)\right)
$$

given by

$$
\left(S(h)_{x} \xi\right)(\gamma)=A_{h} \cdot \xi\left(\left(h^{-1} \gamma\right) \sigma_{h^{-1}}(s(\gamma))\right)
$$


for all $\xi \in L^{2}\left(G_{x}, \Omega^{1 / 2} G_{x} \otimes t^{*}(E)\right)$ and $\gamma \in G_{x}$.

Since the action consists by hypothesis of inner automorphisms, we immediately deduce that

$$
\left(S(h)_{x} \xi\right)(\gamma)=A_{h} \cdot \xi\left(\left(h^{-1} \gamma\right) \sigma_{h^{-1}}(s(\gamma))\right)=A_{h} \cdot \xi\left(\sigma_{h^{-1}}(t(\gamma)) \gamma\right) .
$$

The importance of these operators is explained in the following

Proposition 2.1.14. The family of bounded operators $S(h)=\left\{S(h)_{x}\right\}_{x \in M}$ on the field of Hilbert spaces $L_{\tau}^{2}\left(G, t^{*}(E)\right)$ defines a multiplier of the $C^{*}$-algebra $C_{r}^{*}(G, E)$ of $G$.

Moreover $S(h)$ preserves the dense subalgebra $C_{c}^{\infty}\left(G, E N D(E) \otimes \Omega^{1 / 2} G\right)$.

Proof. We have to show that for every $P \in C_{r}^{*}(G, E)$, the operators $S(h) P$ and $P S(h)$ are again elements of $C_{r}^{*}(G, E)$. Furthermore, if in particular $P \in$ $C_{c}^{\infty}\left(G, E N D(E) \otimes \Omega^{1 / 2} G\right)$, then we claim that $S(h) P \in C_{c}^{\infty}(G, E N D(E) \otimes$ $\left.\Omega^{1 / 2} G\right)$ and $P S(h) \in C_{c}^{\infty}\left(G, E N D(E) \otimes \Omega^{1 / 2} G\right)$.

Let us first show that $S(h)$ is a multiplier of the $C^{*}$-algebra.

Notice that the field $L_{\tau}^{2}\left(G, t^{*}(E)\right)$ gives rise to a right Hilbert $C^{*}$-module $\mathcal{E}_{M, E}$ on the foliation $C^{*}$-algebra $C_{r}^{*}(G)$. Indeed the construction done in [14], Section 7 carries over in the case of a general Lie groupoid).

One can also define a left action of $C_{c}^{\infty}\left(G, E N D(E) \otimes \Omega^{1 / 2} G\right)$ on $\mathcal{E}_{M, E}$ by convolution. This action consists of adjointable endomorphisms of the $C^{*}$-module and it extends to a well-defined left action of the $C^{*}$-algebra $C_{r}^{*}(G, E)$. Moreover, it can be shown that $C_{c}^{\infty}\left(G, E N D(E) \otimes \Omega^{1 / 2} G\right)$ acts as compact operators of the Hilbert $C^{*}$-module $\mathcal{E}_{M, E}$, which implies that $C_{r}^{*}(G, E)$ is contained in the $C^{*}$-algebra of compact operators of $\mathcal{E}_{M, E}$. (We refer, for instance, to 9 for basic definitions).

Now, from Equation 2.18 it is immediate to see that $S(h)$ defines an adjointable endomorphism of $\mathcal{E}_{M, E}$.

Therefore, since the $C^{*}$-algebra of adjointable endomorphisms of $\mathcal{E}_{M, E}$ is canonically isomorphic to the multiplier algebra of the compact ones (see 9], Theorem 13.4.1), we get the result.

Let us now proceed with proving that $S(h)$ preserves the smooth convolution algebra. To prove this we argue as in [7].

Take $k \in C_{c}^{\infty}\left(G, E N D(E) \otimes \Omega^{1 / 2} G\right)$. Recall that $k$ acts on $L_{\tau}^{2}\left(G, t^{*}(E)\right)$ through the regular representation $R=\left\{R_{x}\right\}_{x \in M}$. Let us check that $S(h) \circ R(k) \in$ $C_{c}^{\infty}\left(G, E N D(E) \otimes \Omega^{1 / 2} G\right)$. Then, for any $\xi \in L^{2}\left(G_{x}, t^{*}(E)\right), \gamma \in G_{x}$

$$
\begin{gathered}
\left((S(h) \circ R(k))_{x} \xi\right)(\gamma)=\left(S(h)_{x} \circ R_{x}(k)\right) \xi(\gamma)=\left(S(h)_{x}\left[R_{x}(k) \xi\right]\right)(\gamma)= \\
=A_{h} \cdot\left(R_{x}(k) \xi\right)\left(\sigma_{h^{-1}}(t(\gamma)) \gamma\right)=A_{h} \cdot \int_{G_{x}} k\left(\sigma_{h^{-1}}(t(\gamma)) \gamma \gamma_{1}^{-1}\right) \xi\left(\gamma_{1}\right)= \\
=\int_{G_{x}} A_{h} \cdot k\left(\sigma_{h^{-1}}(t(\gamma)) \gamma \gamma_{1}^{-1}\right) \xi\left(\gamma_{1}\right) .
\end{gathered}
$$

Thus the Schwartz kernel $k_{h}^{l}$ of the operator $S(h) \circ R(k)$ is given by

$$
k_{h}^{l}(\gamma)=\left(A_{h} \otimes i d\right) \cdot k\left(\sigma_{h^{-1}}(t(\gamma)) \gamma\right)
$$


which is obviously smooth. Recall that $A_{h} \otimes i d$ represents the action of $h$ only on the "target part" of $k$ (see Remark 2.2).

Analogously one computes the Schwartz kernel $k_{h}^{r}$ of the operator $R(k) \circ S(h)$, getting the compactly supported smooth section

$$
k_{h}^{r}(\gamma)=\left(i d \otimes A_{h^{-1}}^{*}\right) \cdot k\left(\sigma_{h^{-1}}(h t(\gamma)) h(\gamma)\right),
$$

where $i d \otimes A_{h^{-1}}^{*}$ denotes the action on the "source part" of $k$ (see again Remark 2.2 . This completes the proof.

Notice that the mapping $h \mapsto S(h)$ satisfies the relations $S\left(h_{1} h_{2}\right)=S\left(h_{1}\right) \circ$ $S\left(h_{2}\right)$ and $S(e)=I d$, where $e \in \Gamma$ is the identity element of the group. In other words it defines another unitary representation of $\Gamma$ on the field of Hilbert spaces $L_{\tau}^{2}\left(G, t^{*}(E)\right)$.

Proposition 2.1.15. The pair $(R, S)$ defines a covariant representation of $\left(C_{c}^{\infty}(G, E), \Gamma\right)$ on the field of Hilbert spaces $L_{\tau}^{2}\left(G, t^{*}(E)\right)$. In symbols

$$
R_{x}(h \cdot k)=S(h)_{x} R_{x}(k) S\left(h^{-1}\right)_{x}
$$

for all $h \in \Gamma, k \in C_{c}^{\infty}(G, E), x \in M$.

Proof. The proof is similar to that of Lemma 2.1.12.

Let $\xi \in L^{2}\left(G_{x}, \Omega^{1 / 2} G_{x} \otimes t^{*}(E)\right)$ and $\gamma \in G_{x}$. Then

$$
\begin{gathered}
{\left[S(h)_{x} \circ R_{x}(k) \circ S\left(h^{-1}\right)_{x}\right](\xi)(\gamma)=S(h)_{x}\left[R_{x}(k)\left(S\left(h^{-1}\right)_{x} \xi\right)\right](\gamma)=} \\
=A_{h} \cdot\left(R_{x}(k)\left(S\left(h^{-1}\right)_{x} \xi\right)\right)\left(h^{-1}(\gamma) \sigma_{h^{-1}}(s(\gamma))\right)= \\
=A_{h} \cdot \int_{G_{x}} k\left(h^{-1}(\gamma) \sigma_{h^{-1}}(s(\gamma)) \gamma_{1}^{-1}\right)\left(S\left(h^{-1}\right) \xi\right)\left(\gamma_{1}\right)= \\
=A_{h} \cdot \int_{G_{x}} k\left(h^{-1}(\gamma) \sigma_{h^{-1}}(s(\gamma)) \gamma_{1}^{-1}\right) A_{h}^{-1} \cdot \xi\left(h\left(\gamma_{1}\right) \sigma_{h}\left(s\left(\gamma_{1}\right)\right)\right) .
\end{gathered}
$$

Now, setting $\gamma^{\prime}=h\left(\gamma_{1}\right) \sigma_{h}\left(s\left(\gamma_{1}\right)\right)=\sigma_{h}\left(t\left(\gamma_{1}\right)\right) \gamma_{1}$, we have

$$
\begin{gathered}
\gamma_{1}^{-1}=\left(\sigma_{h}\left(t\left(\gamma_{1}\right)\right)^{-1} \gamma^{\prime}\right)^{-1}= \\
=\left(h^{-1}\left(\gamma^{\prime}\right) \sigma_{h}\left(h^{-1}\left(s\left(\gamma_{1}\right)\right)\right)^{-1}\right)^{-1}=\sigma_{h}\left(h^{-1}\left(s\left(\gamma_{1}\right)\right)\right) h^{-1}\left(\gamma^{\prime}\right)^{-1} .
\end{gathered}
$$

Hence, by invariance of the integral with respect to diffeomorphisms it follows that

$$
\begin{gathered}
A_{h} \cdot \int_{G_{x}} k\left(h^{-1}(\gamma) \sigma_{h^{-1}}(s(\gamma)) \gamma_{1}^{-1}\right) A_{h}^{-1} \cdot \xi\left(h\left(\gamma_{1}\right) \sigma_{h}\left(s\left(\gamma_{1}\right)\right)\right)= \\
=\int_{G_{x}} A_{h} \cdot k\left(h^{-1}(\gamma) \sigma_{h^{-1}}(s(\gamma)) \sigma_{h}\left(h^{-1}\left(s\left(\gamma_{1}\right)\right)\right) h^{-1}\left(\gamma^{\prime}\right)^{-1}\right) A_{h}^{-1} \cdot \xi\left(\gamma^{\prime}\right)
\end{gathered}
$$

Now, notice that $s(\gamma)=s\left(\gamma_{1}\right)=s\left(\gamma^{\prime}\right)$ and that

$$
\sigma_{h^{-1}}(s(\gamma)) \sigma_{h}\left(h^{-1}\left(s\left(\gamma_{1}\right)\right)\right)=\left(\sigma_{h^{-1}} \star \sigma_{h}\right)\left(h^{-1}(s(\gamma))\right)=1_{h^{-1}(s(\gamma))} .
$$


Therefore,

$$
\begin{gathered}
\int_{G_{x}} A_{h} \cdot k\left(h^{-1}(\gamma) \sigma_{h^{-1}}(s(\gamma)) \sigma_{h}\left(h^{-1}\left(s\left(\gamma_{1}\right)\right)\right) h^{-1}\left(\gamma^{\prime}\right)^{-1}\right) A_{h}^{-1} \cdot \xi\left(\gamma^{\prime}\right)= \\
=\int_{G_{x}} A_{h} \cdot k\left(h^{-1}(\gamma) h^{-1}\left(\gamma^{\prime}\right)^{-1}\right) A_{h}^{-1} \cdot \xi\left(\gamma^{\prime}\right)= \\
=\int_{G_{x}}\left[\left(A_{h} \otimes A_{h}^{*}\right) \cdot k\left(h^{-1}(\gamma) h^{-1}\left(\left(\gamma^{\prime}\right)^{-1}\right)\right)\right] \xi\left(\gamma^{\prime}\right)=\int_{G_{x}}(h \cdot k)\left(\gamma\left(\gamma^{\prime}\right)^{-1}\right) \xi\left(\gamma^{\prime}\right)
\end{gathered}
$$

which completes the proof.

Now, if $(M, \mathcal{F}, \Gamma)$ is a foliated $\Gamma$-manifold, the holonomy groupoid $H(\mathcal{F})$ and the twisted holonomy groupoid $H(\mathcal{F}, \Gamma)$ are naturally Lie $\Gamma$-groupoids with actions defined respectively as in Proposition 1.5.2 and by Equation 1.85 Therefore, according with the results of this section we deduce

Proposition 2.1.16. For any $\Gamma$-equivariant Hermitian vector bundle $E$, the $C^{*}$-algebras $C_{r}^{*}(M, \mathcal{F}, E)$ and $C_{r}^{*}(M, \mathcal{F}, \Gamma, E)$ associated respectively to $H(\mathcal{F})$ and $H(\mathcal{F}, \Gamma)$ inherit a $\Gamma$-action that turns them into $\Gamma-C^{*}$-algebras. These actions preserve the smooth convolution subalgebras.

Moreover, the inclusion

$j: C_{c}^{\infty}\left(H(\mathcal{F}), E N D(E) \otimes \Omega^{1 / 2} H(\mathcal{F})\right) \rightarrow C_{c}^{\infty}\left(H(\mathcal{F}, \Gamma), E N D(E) \otimes \Omega^{1 / 2} H(\mathcal{F}, \Gamma)\right)$

defined in 2.11 is $\Gamma$-equivariant.

Proof. The only point which has not been proven yet is the equivariance of the inclusion $j$. For simplicity, let us assume $E$ to be the trivial rank one vector bundle. But, by Proposition 1.5 .8 we know that the inclusion $H(\mathcal{F}) \subseteq H(\mathcal{F}, \Gamma)$ of groupoids is $\Gamma$-equivariant, which implies the result.

The results described so far in this section will be important for constructing the equivariant cyclic cocycles we aim at.

\subsection{Equivariant $\mathrm{K}$-theory and the equivariant analytic index class}

\subsubsection{Equivariant $K$-theory}

There are many ways to define equivariant $K$-theory for $C^{*}$-algebras. One is by means of equivariant Kasparov's bivariant $K K$-theory. This theory is extremely general and allows general actions by locally compact groups (and even more general objects, like groupoids or Hopf algebras). The cycles used to define the equivariant $K K$-groups are the so-called equivariant Kasparov bimodules, which are a wide generalization of equivariant Fredholm modules (see [7]). Unfortunately, it is technically more difficult to construct a pairing between equivariant $K$-theory, defined using equivariant Kasparov bimodules, 
and equivariant cyclic cohomology (see chapter 3 for the latter). One possible way to do it, might be to define an equivariant bivariant cyclic/periodic homology and then an equivariant bivariant Connes-Chern character. Equivariant bivariant periodic homology with respect to actions by locally compact groups has been defined and extensively studied by [46]. However, a general equivariant bivariant Connes-Chern character is still missing.

If one restricts, though, to actions by compact groups, one gets a simple description of equivariant $K$-theory, that is very suitable for defining a pairing between equivariant $K$-theory and equivariant cyclic cohomology. Moreover, this description allows to define the $K_{0}^{G}$-functor for algebras acted upon by the compact group $G$, that are not necessarily $C^{*}$-algebras.

Thus, in this section we will give the basic definitions and state the basic properties of equivariant $K$-theory with respect to compact groups, which will be necessary also in next sections. Unless otherwise specified, all groups, that we will deal with, will be compact and second countable.

Let $G$ denote a compact group and let $(A, G, \alpha)$ be a complete locally convex algebra $A$ over $\mathbb{C}$ endowed with an action of $G$ through the group homomorphism $\alpha: G \rightarrow \operatorname{Aut}(A)$. Since $A$ is a locally convex algebra, we require further the action $\alpha$ to be strongly continuous, i.e. for all $a \in A$, the map $g \mapsto \alpha_{g}(a)$ is continuous (see 2.1.3). We will call $A$ a $G$-algebra.

In the case of a $C^{*}$-algebra $A$, such a triple is often called a noncommutative $C^{*}$-dynamical system, or, equivalently, a covariant system. We will also say that $A$ is a $G-C^{*}$-algebra.

Since $G$ is compact, every unitary representation on a Hilbert space is a direct sum of finite dimensional irreducible unitary representations. Thus it suffices to consider only finite dimensional unitary representations of $G$.

Now, for every finite dimensional unitary representation $\Delta: G \rightarrow \operatorname{End}(V)$, consider the $C^{*}$-algebra $\operatorname{End}(V)$ equipped with the natural action of $G$, given by

$$
g \cdot T=\Delta_{g} T \Delta_{g}^{-1}
$$

for all $T \in \operatorname{End}(V)$. Being $A$ a $G$-algebra, the tensor product $A \otimes \operatorname{End}(V)$ is also naturally a $G$-algebra, with respect to the diagonal action

$$
g \cdot(a \otimes T)=(g a) \otimes(g \cdot T)=(g a) \otimes\left(\Delta_{g} T \Delta_{g}^{-1}\right) .
$$

We consider $G$-invariant idempotents $e \in A \otimes \operatorname{End}(V)$, where $V$ runs through all finite dimensional unitary representations of $G$.

If $\Lambda: G \rightarrow \operatorname{End}(W)$ is another finite dimensional representation and $f \in$ $A \otimes \operatorname{End}(W)$ is a $G$-invariant idempotent, then we shall say that $e$ and $f$ are equivalent if there exist a $G$-invariant element $u \in A \otimes \operatorname{Hom}(V, W)$ and a $G$-invariant element $v \in A \otimes \operatorname{Hom}(W, V)$ such that

$$
v u=e \in A \otimes \operatorname{End}(V), u v=f \in A \otimes \operatorname{End}(W)
$$

This is indeed an equivalence relation. We will write $e \sim_{G} f$.

There is a natural binary operation on the set of $G$-invariant idempotents. In 
fact, if $e \in A \otimes \operatorname{End}(V)$ and $f \in A \otimes \operatorname{End}(W)$ are $G$-invariant idempotents, then we can simply set $e \oplus f \in A \otimes \operatorname{End}(V \oplus W)$ to be the $G$-invariant idempotent $\operatorname{diag}(e, f)$. This operation preserves the equivalence relation $\sim_{G}$ and descends to a binary operation on the set $V_{G}(A)$ of equivalence classes, turning it into an abelian monoid with 1 .

Assume, now, $A$ to be a unital $G$-algebra. Thus

Definition 2.4. The equivariant $K$-theory $K_{0}^{G}(A)$ of $A$ is the Grothendieck group $K\left(V_{G}(A)\right)$.

The group $K_{0}^{G}(A)$ has a richer structure than $K_{0}(A)$. Indeed, let $R[G]$ denote the representation ring of $G$, i.e. the ring of formal differences of equivalence classes of finite-dimensional unitary representations of $G$ with direct sum and tensor product as the ring operations. $R[G]$ is a commutative ring with 1 , given by the trivial one-dimensional representation. It is not difficult to check that $K_{0}^{G}(A)$ has a natural structure of $R[G]$-module, defined by sending the class represented by the $G$-invariant idempotent $e \in A \otimes \operatorname{End}(V)$ to the class given by the idempotent $e \otimes I d_{W} \in A \otimes \operatorname{End}(V \otimes W)$, for any class $[W] \in R[G]$. In particular, if $A=\mathbb{C}$, with the trivial action, then $K_{0}^{G}(\mathbb{C}) \cong R[G]$.

Let us, now, consider the general case, in which $A$ is not necessarily unital. Then, we define the equivariant $K$-theory $K_{0}^{G}(A)$ as in the non-equivariant case. Consider $\mathbb{C}$ with the trivial action and denote by $A^{+}$the unitalization of $A$, with the induced action of $G$, trivially extended on $\mathbb{C}$. Denote by

$$
\begin{array}{r}
\pi: A^{+} \rightarrow \mathbb{C} \\
(a, \lambda) \mapsto \lambda
\end{array}
$$

the natural projection. This clearly is an equivariant homomorphism.

Definition 2.5. The equivariant $K$-theory group $K_{0}^{G}(A)$ for $A$ is the kernel of the map

$$
\pi_{*}: K_{0}^{G}\left(A^{+}\right) \rightarrow K_{0}^{G}(\mathbb{C})
$$

induced by $\pi$.

The two definitions coincide, if $A$ is unital and it can be shown that $K_{0}^{G}$ defines a functor on the category of $G$-algebras in the category of $R[G]$-modules.

Important Remark 2.3. Let us briefly focus on the case in which $A$ is a $G-C^{*}$-algebra. In this case the group $K_{0}^{G}(A)$ enjoys properties similar to those of nonequivariant $K$-theory for $C^{*}$-algebras.

First, we can define $K_{1}^{G}(A)$ in a similar fashion to the nonequivariant case. If the suspension $S A=C_{0}(\mathbb{R}) \otimes A$ of $A$ is given the $G$-action which is extended on $C_{0}(\mathbb{R})$ trivially, then $S A$ is a $G-C^{*}$-algebra. Then

Definition 2.6. The $R[G]$-module $K_{1}^{G}(A)$ is defined as $K_{0}^{G}(S A)$. 
It is possible to define $K_{1}^{G}(A)$ in terms of $G$-invariant invertible elements. See for instance [9], Section 11 and the references therein.

The most important properties of ordinary $K$-theory can be generalized to the equivariant case. In particular, one gets the following results.

Theorem 2.2.1. (stability) Let $A$ be a $G-C^{*}$-algebra and let $\mathcal{K}$ be the $C^{*}$-algebra of compact operators on a separable Hilbert space with the trivial $G$-action. Consider the tensor product $A \otimes \mathcal{K}$ endowed with the diagonal action. Then there exists an isomorphism of $R[G]$-modules

$$
\phi_{*}: K_{0}^{G}(A) \rightarrow K_{0}^{G}(A \otimes \mathcal{K})
$$

Theorem 2.2.2. (Bott periodicity) For any $G-C^{*}$-algebra $A$, there exists a natural $R[G]$-module isomorphism

$$
\beta: K_{0}^{G}(A) \rightarrow K_{1}^{G}(S A)
$$

Theorem 2.2.3. (excission) Let $A$ be a $G-C^{*}$-algebra and let $J$ be a closed two-sided $G$-invariant ideal of $A$. Then, there exist $R[G]$-module homomorphisms

$$
\partial_{0}: K_{1}^{G}(A / J) \rightarrow K_{0}^{G}(J)
$$

and

$$
\partial_{1}: K_{0}^{G}(A / J) \rightarrow K_{1}^{G}(J)
$$

which make the following six-term sequence of $R[G]$-modules exact:

$$
\begin{array}{ccccc}
K_{0}^{G}(J) & \longrightarrow & K_{0}^{G}(A) & \longrightarrow & K_{0}^{G}(A / J) \\
\uparrow \partial_{0} & & & & \downarrow \partial_{1} \\
K_{1}^{G}(A / J) & \longleftarrow & K_{1}^{G}(A) & \longleftarrow & K_{1}^{G}(J)
\end{array}
$$

where the horizontal arrows are induced by the inclusion $J \rightarrow A$ and the quotient $\operatorname{map} A \rightarrow A / J$.

For proofs of these results and further remarks we refer again to [9] and the references therein.

\subsubsection{Pseudo-differential operators, ellipticity and the fun- damental extension}

This section will provide a very brief introduction to the theory of elliptic longitudinal pseudodifferential operators defined on a Lie groupoid. We will begin with the notions of longitudinal differential and pseudodifferential operators. Of course, we are interested in elliptic longitudinal operators, i.e. operators that are " invertible up to smoothing operators". The concept of ellipticity mentioned here is a very important generalization of the classical notion for compact manifolds and it is formulated through a suitable defined principal symbol associated to any longitudinal pseudodifferential operator. This correspondence is a surjective map and gives rise to an extension of $C^{*}$-algebras, that is fundamental for 
defining index classes. Along the nonequivariant case, we will also deal with the equivariant version of the above notions. This paragraph is mainly based upon [21] and [40]. Another important reference is [13], where a pseudodifferential calculus for foliated manifolds was first introduced.

Let $G$ be a Lie groupoid with space of objects $G^{(0)}=M$. For many reasons, both analytic and topological, we assume $M$ to be compact. Moreover for simplicity, we will denote the bundle of half-densities $\Omega^{1 / 2} G$ over $G$ simply by $\Omega^{1 / 2}$ and the bundle of leafwise half-densities over $M$ by $\Omega_{\mathcal{F}}^{1 / 2}$. Finally, let $E, F$ be Hermitian smooth vector bundles over $M$.

For $\gamma \in G$, we consider again the induced operator

$$
U_{\gamma}: C_{c}^{\infty}\left(G_{s(\gamma)}, \Omega^{1 / 2} G_{s(\gamma)} \otimes t^{*}(E)\right) \rightarrow C^{\infty}\left(G_{t(\gamma)}, \Omega^{1 / 2} G_{t(\gamma)} \otimes t^{*}(E)\right),
$$

given by

$$
U_{\gamma}(f)\left(\gamma^{\prime}\right)=f\left(\gamma^{\prime} \gamma\right)
$$

We will deal with families of operators parametrized by the space of objects which are invariant with respect to the action of the groupoid on itself defined by right multiplication. Precisely,

Definition 2.7. A $G$-operator, also called a tangential operator, $P$ is defined by the following data.

1. $P$ is given by a family of operators $P=\left\{P_{x}\right\}_{x \in M}$, where

$$
P_{x}: C_{c}^{\infty}\left(G_{x}, \Omega^{1 / 2} G_{x} \otimes t^{*}(E)\right) \rightarrow C^{\infty}\left(G_{x}, \Omega^{1 / 2} G_{x} \otimes t^{*}(F)\right)
$$

and

$$
(P f)(\gamma)=P_{x} f(\gamma)
$$

for all $f \in C_{c}^{\infty}\left(G_{x}, \Omega^{1 / 2} G_{x} \otimes t^{*}(E)\right), \gamma \in G_{x}$.

2. The operator $P$ is $G$-invariant, i.e. $\forall \gamma \in G$,

$$
U_{\gamma} P_{s(\gamma)}=P_{t(\gamma)} U_{\gamma}
$$

If $P$ is a $G$-operator, we denote by $k_{x} \in C^{-\infty}\left(G_{x} \times G_{x},\left(\Omega^{1 / 2} G_{x} \otimes t^{*}(F)\right) \otimes\right.$ $\left.\left(\Omega^{1 / 2} G_{x} \otimes t^{*}(E)^{*}\right)\right)$ the Schwartz kernel of the operator $P_{x}$, for any $x \in M$, so that

$$
P_{x} f(\gamma)=\int_{G_{x}} k_{x}\left(\gamma, \gamma^{\prime}\right) f\left(\gamma^{\prime}\right)
$$

for all $f \in C_{c}^{\infty}\left(G_{x}, \Omega^{1 / 2} G_{x} \otimes t^{*}(E)\right), \gamma \in G_{x}$. Using (1) and (2), one immediately gets the following equality of vector-valued distributions

$$
k_{x}\left(\gamma_{1} \gamma, \gamma_{2}\right)=k_{y}\left(\gamma_{1}, \gamma_{2} \gamma^{-1}\right)
$$

for every $\gamma \in G$ and $s(\gamma)=x, t(\gamma)=y$. Thus, setting $k_{P}(\gamma)=k_{s(\gamma)}(\gamma, s(\gamma))$, one gets $k_{x}\left(\gamma, \gamma^{\prime}\right)=k_{P}\left(\gamma\left(\gamma^{\prime}\right)^{-1}\right)$ and that

$$
P f(\gamma)=\int_{G_{x}} k_{P}\left(\gamma\left(\gamma^{\prime}\right)^{-1}\right) f\left(\gamma^{\prime}\right)
$$


Thus, $k_{P}$ can be viewed as a single distribution on the groupoid $G$ with values in the bundle $\Omega^{1 / 2} \otimes s^{*}\left(E^{*}\right) \otimes t^{*}(F)$ acting on sections by convolution. For the computations we have omitted we refer to [21].

With the distributional kernel $k_{P}$ at hand, it is simple to make precise which growth conditions the $G$-operators have to satisfy. Thus, we say that $P$ is uniformly supported if its Schwartz kernel $k_{P}$ has compact support.

Moreover

Definition 2.8. A $G$-operator is called smoothing, if its distributional kernel $k_{P}$ lies in $C^{\infty}\left(G, \Omega^{1 / 2} \otimes s^{*}\left(E^{*}\right) \otimes t^{*}(F)\right)$.

In the following, we will only deal with uniformly supported $G$-operators. A uniformly supported smoothing operator will be also called compactly smoothing.

Remark 2.3. If the $G$-operator $P$ is compactly smoothing, then its Schwartz kernel $k_{P}$ belongs to $C_{c}^{\infty}\left(G, \Omega^{1 / 2} \otimes s^{*}\left(E^{*}\right) \otimes t^{*}(F)\right)$ and it acts on half densities by convolution. If $E=F$, we have simply re-obtained formula 2.8 In other words, the $G$-operator $P=\left\{P_{x}\right\}_{x \in M}$ coincides with the family of operators $\left\{R_{x}\left(k_{P}\right)\right\}_{x \in M}$ restricted to smooth sections.

Example 2.4. Let $\pi: M \rightarrow B$ be a smooth fibre bundle with connected fibers and $G=M \times{ }_{B} M$ its holonomy groupoid (see 1.9 for the details). It is evident that each $s$-fiber $G_{x}$ can be identified with the fiber $\pi^{-1}(\pi(x))$ of the fiber bundle $\pi$.

Now Axiom (2) in 2.7 implies that, for each $M \times_{B} M$-operator $P$ and for any $x, y \in M$ with $\pi(x)=\pi(y$,$) i.e. lying in the same fiber, one has P_{x}=P_{y}$. Hence, a $M \times_{B} M$-operator is given by a family of operators $\left\{\widetilde{P}_{b}\right\}_{b \in B}$, parametrized by the base manifold $B$, with the relation $P_{x}=\widetilde{P}_{\pi(x)}$.

Let us, now, define pseudodifferential operators on a Lie groupoid.

Definition 2.9. Let $G$ be a Lie groupoid over $M$ and let $E, F$ be complex smooth vector bundles on $M$. A compactly supported pseudodifferential $G$-operator $P$ of order $m$ is a uniformly supported $G$-operator such that

1. its Schwartz kernel $k_{P}$ is smooth outside $M$, and

2. for any distinguished chart $\phi: U \subseteq G \rightarrow L \times s(U)$ of $G$ and any $\chi \in$ $C_{c}^{\infty}(U)$, the operator

$$
\left(\phi^{-1}\right)^{*}(\chi P \chi) \phi^{*}: C_{c}^{\infty}\left(L \times s(U), \Omega^{1 / 2} L \otimes t^{*}(E)\right) \rightarrow C^{\infty}\left(L \times s(U), \Omega^{1 / 2} L \otimes t^{*}(F)\right)
$$

is a smooth family of classical pseudodifferential operators of order $m$ parametrized by $s(U)$.

Thus, a pseudodifferential operator is a $G$-invariant family of operators that are locally classical pseudodifferential operators.

In particular, a differential operator on the Lie groupoid $G$ is described by a $G$-invariant family of differential operators along the $s$-fibers. 
We will denote by $\Psi_{c}^{m}(G ; E, F)$ the space of uniformly supported pseudodifferential $G$-operators of order $m$ on $G$ between the vector bundles $E$ and $F$ and we set $\Psi_{c}^{\infty}(G ; E, F)=\bigcup_{m \in \mathbb{Z}} \Psi_{c}^{m}(G ; E, F)$.

If $E=F$, it turns out (see [42]) that $\Psi_{c}^{\infty}(G ; E)$ is actually a filtered algebra with respect to the composition. Moreover, if $E \otimes \Omega_{\mathcal{F}}^{1 / 2}$ is a Hermitian vector bundle and if we assign to any operator $P \in \Psi_{c}^{\infty}(G ; E)$ its formal adjoint $P^{*}$, then $P^{*}$ is still a pseudodifferential $G$-operator of the same order of $P$ and in this way one defines on $\Psi_{c}^{\infty}(G ; E)$ an involution compatible with the product. Thus, $\Psi_{c}^{\infty}(G ; E)$ is a $*$-algebra.

Important $*$-subalgebras of $\Psi_{c}^{\infty}(G ; E)$ are provided by $\Psi_{c}^{0}(G ; E)$, the algebra of order 0 pseudodifferential $G$-operators, and $\Psi_{c}^{-\infty}(G ; E)$. Actually, $\Psi_{c}^{-\infty}(G ; E)$ is a two sided ideal of $\Psi_{c}^{\infty}(G ; E)$ (see [42]).

The following result is immediate for uniformly supported pseudodifferential $G$-operators.

Lemma 2.2.4. Let $G$ be a Lie groupoid and $H$ an open subgroupoid of $G$, both over the manifold $M$. Let $E$ be a complex vector bundle on $M$. Then the inclusion $H \subseteq G$ induces a filtration preserving algebra homomorphism

$$
i: \Psi_{c}^{\infty}(H ; E) \hookrightarrow \Psi_{c}^{\infty}(G ; E)
$$

defined by extending to the whole $G$ the convolution kernel $k_{P}$ of $P \in \Psi_{c}^{\infty}(H ; E)$ by zero outside the support.

As in the classical situation provided by pseudodifferential operators on a compact manifold, also for a pseudodifferential $G$-operator on a Lie groupoid $G$, one can define a principal symbol, that generalizes the classical one.

Let $\pi: \mathfrak{g}^{*} \rightarrow M$ be the dual Lie algebroid of $G$. Now, for any $P \in \Psi_{c}^{m}(G, E)$, the principal symbol of $P$ is defined as the map

$$
\sigma_{p r}(P): \mathfrak{g}^{*} \backslash\{0\} \rightarrow \operatorname{End}\left(\pi^{*}\left(E \otimes \Omega_{\mathcal{F}}^{1 / 2}\right)\right)
$$

by the formula

$$
\sigma_{p r}(P)(x, \xi)=\sigma_{p r}\left(P_{x}\right)(x, \xi)
$$

for every $\xi \in \mathfrak{g}_{x}^{*}, x \in M$. Here $\sigma_{p r}\left(P_{x}\right)$ denotes the principal symbol of the classical pseudodifferential operator $P_{x}$ on $G_{x}$. Note that, choosing an Euclidean metric on $\mathfrak{g}^{*}$ and using homogeneity, the principal symbol $\sigma_{p r}(P)$ can actually be defined on the "co-sphere bundle" $S^{*} \mathfrak{g}$ of $G$, i.e. the set of vectors of $\mathfrak{g}^{*} \backslash\{0\}$ of lenght 1. In what follows we will always think of the principal symbol as to be defined on $S^{*} \mathfrak{g}$.

To prove that the definition 2.40 is well-posed, there is something to check, namely that there are representatives $a_{x}$ of $\sigma_{p r}\left(P_{x}\right)$ such that the family $\left\{a_{x}\right\}$ is smooth and invariant. Moreover, the principal symbol is compatible with the composition of operators, i.e. $\sigma_{p r}(P Q)=\sigma_{p r}(P) \sigma_{p r}(Q)$. All the details can be found in [42] and we omit them here. 
Definition 2.10. Let $P \in \Psi_{c}^{m}(G ; E)$. We will say that $P$ is elliptic if its principal symbol $\sigma_{p r}(P)$ is invertible for all $(x, \xi) \in S^{*} \mathfrak{g}$.

We get, also in this context, the following fundamental result.

Theorem 2.2.5. Let $P \in \Psi_{c}^{m}(G ; E)$ be an elliptic pseudodifferential $G$-operator of order $m$. Then, there exists an elliptic pseudodifferential $G$-operator $Q \in$ $\Psi_{c}^{-m}(G ; E)$ of order $-m$ such that $1-Q P=S_{0}$ and $1-P Q=S_{1}$ are compactly smoothing operators.

The pseudodifferential operator $Q$ is called a parametrix or a quasi-inverse of $P$.

Proof. A proof of this result can be found in [45, Proposition 3.5.1.

Recall that a pseudodifferential $G$-operator $P \in \Psi_{c}^{0}(G ; E)$ extends to an intertwining bounded operator

$$
P: L_{\tau}^{2}\left(G, t^{*}(E)\right) \rightarrow L_{\tau}^{2}\left(G, t^{*}(E)\right)
$$

One may norm $P$ setting

$$
\|P\|=\sup _{x \in M}\left\|P_{x}\right\|
$$

where

$$
P_{x}: L^{2}\left(G_{x}, \Omega^{1 / 2} G_{x} \otimes t^{*}(E)\right) \rightarrow L^{2}\left(G_{x}, \Omega^{1 / 2} G_{x} \otimes t^{*}(E)\right)
$$

is the pseudodifferential operator at the point $x \in M$.

Consider, now, the $*$-algebra $\Psi_{c}^{0}(G ; E)$ normed as above and take the closure. Thus one gets a $C^{*}$-algebra $\overline{\Psi_{c}^{0}}(G ; E)$, called the pseudodifferential $C^{*}$-algebra. Clearly, $\Psi_{c}^{-\infty}(G ; E) \cong C_{c}^{\infty}\left(G, E N D(E) \otimes \Omega^{1 / 2} G\right)$ is contained in $\overline{\Psi_{c}^{0}}(G ; E)$. Now, by Remark 2.3 and observing that the norm above coincides with 2.10 one immediately gets that $C_{r}^{*}(G, E)$ is a $C^{*}$-subalgebra of $\overline{\Psi_{c}^{0}}(G ; E)$.

Actually, one has the following result.

Theorem 2.2.6. Let $G$ be a Lie groupoid over $M$ and let $E$ be a Hermitian vector bundle on $M$.

1. One has that

$$
0 \rightarrow \Psi_{c}^{-1}(G ; E) \rightarrow \Psi_{c}^{0}(G ; E) \stackrel{\sigma_{p r}}{\longrightarrow} C^{\infty}\left(S^{*} \mathfrak{g}, \operatorname{End}\left(\pi^{*}\left(E \otimes \Omega_{\mathcal{F}}^{1 / 2}\right)\right)\right) \rightarrow 0
$$

is exact.

2. The principal symbol map extends to a surjective symbol map

$$
\sigma_{p r}: \overline{\Psi_{c}^{0}}(G ; E) \rightarrow C\left(S^{*} \mathfrak{g}, \operatorname{End}\left(\pi^{*}\left(E \otimes \Omega_{\mathcal{F}}^{1 / 2}\right)\right)\right)
$$

such that the sequence of $C^{*}$-algebras

$$
0 \rightarrow C_{r}^{*}(G, E) \rightarrow \overline{\Psi_{c}^{0}}(G ; E) \stackrel{\sigma_{p r}}{\longrightarrow} C\left(S^{*} \mathfrak{g}, \operatorname{End}\left(\pi^{*}\left(E \otimes \Omega_{\mathcal{F}}^{1 / 2}\right)\right)\right) \rightarrow 0
$$

is exact. 
The proof of the first statement can be found in [42. For the second one, the proof given in [40] in the case of the holonomy groupoid carries over in the context of general Lie groupoids.

The above result allows us to extend the notion of ellipticity to any operator $P \in \overline{\Psi_{c}^{0}}(G ; E)$ by simply declaring $P$ to be elliptic if its principal symbol $\sigma_{p r}(P) \in C\left(S^{*} \mathfrak{g}, \operatorname{End}\left(\pi^{*}\left(E \otimes \Omega_{\mathcal{F}}^{1 / 2}\right)\right)\right)$ is invertible for all $x \in S^{*} \mathfrak{g}$.

Let us consider, now, the case of a Lie groupoid endowed with additional symmetries given by the action of a group.

Thus, let $\Gamma$ denote a compact Lie group acting on the Lie groupoid $G$, as defined in 1.29. Recall that if $E$ is a $\Gamma$-equivariant Hermitian vector bundle over the space of objects $G^{(0)}=M$, then the pull-back bundle $t^{*}(E)$ inherits a natural action of $\Gamma$, that makes it into a $\Gamma$-equivariant bundle on $G$. Thus, it remains induced a natural action of $\Gamma$ on the field $\left\{C_{c}^{\infty}\left(G_{x}, \Omega^{1 / 2} G_{x} \otimes t^{*}(E)\right)\right\}_{x \in M}$, defined through the linear isomorphisms

$$
U_{h}: C_{c}^{\infty}\left(G_{x}, \Omega^{1 / 2} G_{x} \otimes t^{*}(E)\right) \rightarrow C_{c}^{\infty}\left(G_{h(x)}, \Omega^{1 / 2} G_{h(x)} \otimes t^{*}(E)\right)
$$

by

for all $x \in M, h \in H$.

$$
\left(U_{h} s\right)(\gamma)=h \cdot s\left(h^{-1}(\gamma)\right)
$$

Let, now, $P \in \Psi_{c}^{m}(G ; E)$. For any $h \in \Gamma$ and $x \in M$, we define the operator

$(h \cdot P)_{x}=U_{h^{\circ}} \circ P_{h^{-1} x} \circ U_{h^{-1}}: C_{c}^{\infty}\left(G_{x}, \Omega^{1 / 2} G_{x} \otimes t^{*}(E)\right) \rightarrow C_{c}^{\infty}\left(G_{x}, \Omega^{1 / 2} G_{x} \otimes t^{*}(E)\right)$.

The new operator $h \cdot P$ can be proven to be a pseudodifferential $G$-operator. Indeed, since this is a local statement, it is a consequence of the Theorem 3.12 in [36] about the behaviour of pseudodifferential operators under change of coordinates of the underlying manifold.

Thus, Equation 2.45 ensures that the space of pseudodifferential $G$-operators $\Psi_{c}^{m}(G ; E)$ admits an action by $\Gamma$.

In particular, $\Psi_{c}^{0}(G ; E)$ is a $\Gamma$-algebra with the $\Gamma$-invariant ideal $\Psi_{c}^{-1}(G ; E)$.

The principal symbol map

$$
\sigma_{p r}: \Psi_{c}^{0}(G ; E) \rightarrow C^{\infty}\left(S^{*} \mathfrak{g}, \operatorname{End}\left(\pi^{*}\left(E \otimes \Omega_{\mathcal{F}}^{1 / 2}\right)\right)\right)
$$

turns out to be $\Gamma$-equivariant. In fact, the dual Lie algebroid $\mathfrak{g}^{*}$ inherits an action by Lie algebroid morphisms, induced by the action of $\Gamma$ on $G$ (see 38 . for details). Moreover, choosing a $\Gamma$-invariant metric on the co-sphere bundle $S^{*} \mathfrak{g}$, it follows that this action descends to $S^{*} \mathfrak{g}$.

Hence, the space $C^{\infty}\left(S^{*} \mathfrak{g}, \operatorname{End}\left(\pi^{*}\left(E \otimes \Omega_{\mathcal{F}}^{1 / 2}\right)\right)\right)$ is naturally endowed with an action of $\Gamma$.

One has

$\sigma_{p r}(h \cdot P)(x, \xi)=\sigma_{p r}\left((h \cdot P)_{x}\right)(x, \xi)=h\left[\sigma_{p r}\left(P_{h^{-1}(x)}\right)(h(x, \xi))\right]=\left(h \cdot \sigma_{p r}\right)(x, \xi)$

where in the second equation we have used again Theorem 3.12 and Theorem 3.5 in [36] to have an explicit formula for the principal symbol of $h \cdot P$ in terms of that of $P$. 
Proposition 2.2.7. If $G$ is a Lie $\Gamma$-groupoid, then the extension

$$
0 \rightarrow \Psi_{c}^{-1}(G ; E) \rightarrow \Psi_{c}^{0}(G ; E) \stackrel{\sigma_{p r}}{\longrightarrow} C^{\infty}\left(S^{*} \mathfrak{g}, \operatorname{End}\left(\pi^{*}\left(E \otimes \Omega_{\mathcal{F}}^{1 / 2}\right)\right)\right) \rightarrow 0
$$

is an extension of $\Gamma$-algebras and $\Gamma$-equivariant homomorphisms.

Moreover, taking closures with respect to the norm 2.41. one gets the extension of $\Gamma-C^{*}$-algebras

$$
0 \rightarrow C_{r}^{*}(G, E) \rightarrow \overline{\Psi_{c}^{0}}(G ; E) \stackrel{\sigma_{p r}}{\longrightarrow} C\left(S^{*} \mathfrak{g}, \operatorname{End}\left(\pi^{*}\left(E \otimes \Omega_{\mathcal{F}}^{1 / 2}\right)\right)\right) \rightarrow 0
$$

Proof. This is a consequence of Theorem 2.2.6, the discussion above and the fact that the $\Gamma$-action on $\Psi_{c}^{0}(G ; E)$ extends to a strongly continuous action on the $C^{*}$-algebra $\overline{\Psi_{c}^{0}}(G ; E)$.

Definition 2.11. Let $G$ be a Lie $\Gamma$-groupoid and let $P \in \Psi_{c}^{m}(G ; E)$. We say that $P$ is $\Gamma$-invariant if $h \cdot P=P$, for all $h \in \Gamma$.

Since the principal symbol map is $\Gamma$-equivariant, it follows that the principal symbol of a $\Gamma$-invariant pseudodifferential $G$-operator $P$ is an invariant section. If the operator $P$ is also elliptic, then one can choose a $\Gamma$-invariant parametrix $Q$ of $P$. This will be important in the next section to define the equivariant analitic index class $i n d_{a}^{\Gamma}(P)$ of a $\Gamma$-invariant elliptic pseudodifferential $G$-operator $P$.

One gets the following equivariant version of 2.2 .4

Lemma 2.2.8. Let $G$ be a Lie $\Gamma$-groupoid and $H$ a $\Gamma$-invariant open subgroupoid of $G$, both over the manifold $M$. Then the inclusion $H \subseteq G$ induces a $\Gamma$-equivariant filtration-preserving algebra monomorphism

$$
i: \Psi_{c}^{\infty}(H ; E) \hookrightarrow \Psi_{c}^{\infty}(G ; E)
$$

defined again by extending to the whole $G$ the convolution kernel $k_{P}$ of $P \in$ $\Psi_{c}^{\infty}(H ; E)$ by zero outside the support.

Proof. Take $P \in \Psi_{c}^{\infty}(H ; E)$ with distributional kernel $k_{P}$. Since the action on $H$ is the restriction of that on $G$, it is the same letting $h \in \Gamma$ act on $k_{P}$ and then extending the resulting distribution to $G$ or, conversely, extending first $k_{P}$ to $G$ and then acting on it with $h$.

Let, now, $(M, \mathcal{F})$ be a foliated manifold and $E$ is a Hermitian vector bundle over $M$. The pseudodifferential calculus along the leaves of the foliation is by definition the algebra $\Psi_{c}^{\infty}(H(\mathcal{F}) ; E)$ associated to the holonomy groupoid $H(\mathcal{F})$ of the foliation. Since $H(\mathcal{F})$ is a Lie groupoid for any foliation $(M, \mathcal{F})$, all the results of this section hold in the particular case of $\Psi_{c}^{\infty}(H(\mathcal{F}) ; E)$. In particular, one can talk about differential and pseudodifferential operators along the leaves of the foliation, principal symbols and elliptic pseudodifferential operators. Furthermore, one has the foliated extension given in Theorem 2.2.6 relative to the holonomy groupoid, that is important to define the analytic index class of an elliptic longitudinal operator. 
If $(M, \mathcal{F}, \Gamma)$ is a foliated $\Gamma$-manifold and $H(\mathcal{F}, \Gamma)$ its twisted holonomy groupoid, then we define the pseudodifferential calculus of $(M, \mathcal{F}, \Gamma)$ to be the algebra $\Psi_{c}^{\infty}(H(\mathcal{F}, \Gamma) ; E)$ of uniformly supported pseudodifferential $H(\mathcal{F}, \Gamma)$-operators. $\Psi_{c}^{\infty}(H(\mathcal{F}, \Gamma) ; E)$ admits an action of $\Gamma$ coming from the action of $\Gamma$ on $H(\mathcal{F}, \Gamma)$. Moreover, since by Proposition 1.4 .3 and Proposition 1.5 .8 the holonomy groupoid $H(\mathcal{F})$ is a $\Gamma$-invariant open Lie subgroupoid of $H(\mathcal{F}, \Gamma)$, one has the inclusion

$$
j: \Psi_{c}^{\infty}(H(\mathcal{F}) ; E) \hookrightarrow \Psi_{c}^{\infty}(H(\mathcal{F}, \Gamma) ; E)
$$

which, by Lemma 2.2 .8 , is a $\Gamma$-equivariant filtration-preserving algebra monomorphism.

Since $\Psi_{c}^{-\infty}(G ; E) \cong C_{c}^{\infty}\left(G, E N D(E) \otimes \Omega^{1 / 2} G\right)$ for any Lie groupoid, it is clear that the inclusion 2.47 extends the inclusion

$j: C_{c}^{\infty}\left(H(\mathcal{F}), E N D(E) \otimes \Omega^{1 / 2} H(\mathcal{F})\right) \rightarrow C_{c}^{\infty}\left(H(\mathcal{F}, \Gamma), E N D(E) \otimes \Omega^{1 / 2} H(\mathcal{F}, \Gamma)\right)$

between compactly smoothing pseudodifferential operators.

\subsubsection{The equivariant index class and the Lefschetz class}

Using the notions introduced in the previous paragraphs, we are now going to define the equivariant analytic index class of an elliptic $\Gamma$-invariant pseudodifferential $G$-operator, where $G$ is a Lie $\Gamma$-groupoid. We will first define the index of operators of order 0 using the general extension of $\Gamma-C^{*}$-algebras 2.2.7. Looking at this definition carefully, we will realize that the index class can be defined as a $K^{\Gamma}$-theory class of the smooth convolution algebra $C_{c}^{\infty}(G)$ of the groupoid and that this construction of the index class generalizes immediately to the case of operators of arbitrary order.

Besides, we will also introduce the Lefschetz class relative to the operator. This is an invariant both of the operator and of a chosen element of $\Gamma$ and can be viewed as a localization of the equivariant index class to the fixed-point set of this element. The original idea to interpret Lefschetz-type invariants of compact smooth manifolds in terms of equivariant indices goes back to the fundamental work of Atiyah, Singer and Segal [4], 3]. These ideas have been generalized to the realm of foliated manifolds by Benameur in [5].

Since from now on we will constantly use the equivariant $K$-theory groups, previously introduced, we will always assume that the Lie groupoids have compact space of objects and the groups acting on them are compact Lie groups.

Let $G$ be a Lie $\Gamma$-groupoid and let $E$ a Hermitian $\Gamma$-equivariant vector bundle on the space of objects $M$. Take $P \in \overline{\Psi_{c}^{0}}(G ; E) \Gamma$-invariant and elliptic. Then, by construction, the principal symbol $\sigma_{p r}(P)$ is an invertible $\Gamma$-invariant element of the $C^{*}$-algebra $C\left(S^{*} \mathfrak{g}, \operatorname{End}\left(\pi^{*}(E)\right)\right)$ and thus defines naturally an element

$$
\left[\sigma_{p r}(P)\right] \in K_{1}^{\Gamma}\left(C\left(S^{*} \mathfrak{g}, \operatorname{End}\left(\pi^{*}(E)\right)\right)\right) .
$$

By Proposition 2.2.7, we have the extension of $\Gamma-C^{*}$-algebras

$$
0 \rightarrow C_{r}^{*}(G, E) \rightarrow \overline{\Psi_{c}^{0}}(G ; E) \stackrel{\sigma_{p r}}{\longrightarrow} C\left(S^{*} \mathfrak{g}, \operatorname{End}\left(\pi^{*}(E)\right)\right) \rightarrow 0
$$


Applying Theorem 2.2.3 to this extension, one gets a long exact sequence in equivariant $K$-theory and, in particular, the boundary homomorphism

$$
\partial_{0}: K_{1}^{\Gamma}\left(C\left(S^{*} \mathfrak{g}, \operatorname{End}\left(\pi^{*}(E)\right)\right)\right) \rightarrow K_{0}^{\Gamma}\left(C_{r}^{*}(G, E)\right)
$$

Definition 2.12. Let $P \in \overline{\Psi_{c}^{0}}(G ; E)$ an elliptic $\Gamma$-invariant operator. The equivariant analytic index of $P$ is the $K_{0}^{\Gamma}$-theory class

$$
\operatorname{ind}_{a}^{\Gamma}(P)=\partial_{0}\left(\left[\sigma_{p r}(P)\right]\right) \in K_{0}^{\Gamma}\left(C_{r}^{*}(G, E)\right) .
$$

Looking at the definition of the equivariant index class more carefully, one gets an explicit representative of the index class in terms of a parametrix in the following way.

Let $Q \in \overline{\Psi_{c}^{0}}(G ; E)$ be a parametrix for $P$. Since $P$ is $\Gamma$-invariant, one can choose a $\Gamma$-invariant parametrix $Q$. In this case, the operators $P Q-1$ and $Q P-1$ are both elements of $C_{r}^{*}(G, E)$ and are $\Gamma$-invariant, as well.

Define the matrix

$$
T=\left(\begin{array}{cc}
P+(1-P Q) P & P Q-1 \\
1-Q P & Q
\end{array}\right) \in M_{2}\left(\overline{\Psi_{c}^{0}}(G ; E)\right)
$$

Then $T$ is invertible with inverse

$$
T^{-1}=\left(\begin{array}{cc}
Q & 1-Q P \\
P Q-1 & P+P(1-Q P)
\end{array}\right)
$$

Letting now $e$ be the $\Gamma$-invariant idempotent $\left(\begin{array}{ll}1 & 0 \\ 0 & 0\end{array}\right) \in M_{2}\left(C_{r}^{*}(G, E)^{+}\right)$, where $C_{r}^{*}(G, E)^{+}$is the unitalization of $C_{r}^{*}(G, E)$, one gets

$$
\operatorname{ind}_{a}^{\Gamma}(P)=\partial_{0}\left(\left[\sigma_{p r}(P)\right]\right)=\left[T e T^{-1}\right]-[e] \in K_{0}^{\Gamma}\left(C_{r}^{*}(G, E)\right)
$$

The second equality above is exactly the definition of the boundary homomorphism $\partial_{0}$ (see, for instance, [9]).

Thus, we have found a representative of the equivariant index class, explicitly given by the $\Gamma$-invariant idempotent

$$
T e T^{-1}=\left(\begin{array}{cc}
1-S_{1}^{2} & \left(S_{1}+S_{1}^{2}\right) P \\
S_{0} Q & S_{0}^{2}
\end{array}\right)
$$

where $S_{0}=1-Q P$ and $S_{1}=1-P Q$.

If $P \in \Psi_{c}^{0}(G ; E)$ is a $\Gamma$-invariant elliptic pseudodifferential $G$-operator of order 0 , one can construct a more refined equivariant index class. More precisely, if $P \in \Psi_{c}^{0}(G ; E)$ is a $\Gamma$-invariant elliptic pseudodifferential $G$-operator, then $P Q-1$ and $Q P-1$ can be chosen to be $\Gamma$-invariant elements of the involutive algebra $\Psi_{c}^{-\infty}(G, E)$.

Using that $\Psi_{c}^{-\infty}(G, E) \cong C_{c}^{\infty}\left(G, E N D(E) \otimes \Omega^{1 / 2} G\right)$ and following the same recipe as above, one gets therefore that the $\Gamma$-invariant idempotents $e$ and 
$T e T^{-1}$ belong to $M_{2}\left(C_{c}^{\infty}\left(G, E N D(E) \otimes \otimes \Omega^{1 / 2} G\right)^{+}\right)$.

One defines the smooth equivariant index class to be

$$
\operatorname{ind}_{C^{\infty}}^{\Gamma}(P)=\left[T e T^{-1}\right]-[e] \in K_{0}^{\Gamma}\left(C_{c}^{\infty}\left(G, E N D(E) \otimes \Omega^{1 / 2} G\right)\right)
$$

Note that in the definition of the smooth equivariant index class, the order of $P$ has not played any significant role. What we have really used, is the crucial fact stated in Theorem 2.2.5 that an elliptic pseudodifferential operator is invertible modulo $\Psi_{c}^{-\infty}(G ; E)$, result that holds for an elliptic operator of arbitrary order. Thus, let $P \in \Psi_{c}^{m}(G ; E)$ be an elliptic $\Gamma$-invariant pseudodifferenatial $G$-operator of order $m>0$ and choose a $\Gamma$-invariant parametrix $Q \in \Psi_{c}^{-m}(G ; E)$ for it. Then, $P Q-1$ and $Q P-1$ are $\Gamma$-invariant operators belonging to $\Psi_{c}^{-\infty}(G, E)$ and we can define again the invertible matrix

$$
T=\left(\begin{array}{cc}
P+(1-P Q) P & P Q-1 \\
1-Q P & Q
\end{array}\right) \in M_{2}\left(\Psi_{c}^{\infty}(G ; E)\right)
$$

and the corresponding $\Gamma$-invariant idempotent

$$
T e T^{-1}=\left(\begin{array}{cc}
1-S_{1}^{2} & \left(S_{1}+S_{1}^{2}\right) P \\
S_{0} Q & S_{0}^{2}
\end{array}\right)
$$

The idempotents $e$, defined as above, and $T e T^{-1}$ belong to the matrix algebra $M_{2}\left(C_{c}^{\infty}\left(G, E N D(E) \otimes \Omega^{1 / 2} G\right)^{+}\right)$and we can give the following

Definition 2.13. Let $P \in \Psi_{c}^{m}(G ; E)$ elliptic and $\Gamma$-invariant. The smooth equivariant index class of $P$ is the $K_{0}^{\Gamma}$-theory class defined by

$$
\operatorname{ind}_{C^{\infty}}^{\Gamma}(P)=\left[T e T^{-1}\right]-[e] \in K_{0}^{\Gamma}\left(C_{c}^{\infty}\left(G, E N D(E) \otimes \Omega^{1 / 2} G\right)\right)
$$

Remark 2.4. It can be proven that this definition does not depend on the choice of the parametrix $Q$ of $P$. See, for instance, 9, Section 8.3.

The inclusion $i: C_{c}^{\infty}\left(G, E N D(E) \otimes \Omega^{1 / 2} G\right) \hookrightarrow C_{r}^{*}(G, E)$ induces an additive map of equivariant $K$-theory groups

$$
i_{*}: K_{0}^{\Gamma}\left(C_{c}^{\infty}\left(G, E N D(E) \otimes \Omega^{1 / 2} G\right)\right) \rightarrow K_{0}^{\Gamma}\left(C_{r}^{*}(G, E)\right) .
$$

and one defines the $C^{*}$-algebraic equivariant index class to be

$$
\operatorname{ind}_{a}^{\Gamma}(P)=i_{*}\left(i n d_{C^{\infty}}^{\Gamma}(P)\right) \in K_{0}^{\Gamma}\left(C_{r}^{*}(G, E)\right) .
$$

for any $P \in \Psi_{c}^{m}(G, E)$. In the case $P \in \Psi_{c}^{0}(G, E)$, it follows by construction that the smooth equivariant index class is mapped to the $C^{*}$-algebraic one as defined in 2.12

In passing from the smooth to the $C^{*}$-algebraic index, we lose informations, since the map $i_{*}$ is not in general injective. However, the $C^{*}$-algebraic index class is a more stable object, satisfying homotopy invariance and vanishing results. For further comments and results about this point we refer to [17] and the references therein. 
Both the smooth and the $C^{*}$-algebraic equivariant index classes are very complicated invariants associated to the Lie $\Gamma$-groupoid and the operator. A very interesting idea, due to Atiyah, Segal and Singer, to extract geometric informations out of them, consists in localizing the equivariant index class to a suitable prime ideal of the representation ring $R[\Gamma]$.

More precisely, recall first that every commutative ring $R$ with unit can be localized at a prime ideal $\mathfrak{p}$ to yield a ring of fractions denoted by $R_{\mathfrak{p}}$. In the same way, every $R$-module $M$ can be localized at the prime ideal $\mathfrak{p}$, giving rise to a $R_{\mathfrak{p}}$-module $M_{\mathfrak{p}}$. The localization procedure defines a functor from the category of $R$-modules to the category of $R_{\mathfrak{p}}$-modules, which is exact. For details and further comments, we refer to [2].

Let, now, $h \in \Gamma$, denote by $[h]$ the conjugacy class of $h$ in $\Gamma$ and let

$$
I_{[h]}=\left\{\chi \in R[\Gamma] \mid \chi\left(g h g^{-1}\right)=0, \quad \forall g \in \Gamma\right\} \subseteq R[\Gamma]
$$

be the prime ideal of the representation ring $R[\Gamma]$ associated with the conjugacy class $[h]$ of $h$. In our case, localization of the representation ring $R[\Gamma]$ at $I_{[h]}$ gives rise to the ring of fractions denoted by $R[\Gamma]_{I_{[h]}}$. As we have seen in Section 2.2.1 the equivariant $K_{0}^{\Gamma}$-theory group of a $\Gamma$-algebra is an example of a $R[\Gamma]$-module. Thus, we can localize the $R[\Gamma]$-module $K_{0}^{\Gamma}\left(C_{c}^{\infty}(G, E)\right)$ at $I_{[h]}$ to get the module of fractions $K_{0}^{\Gamma}\left(C_{c}^{\infty}(G, E)\right)_{[h]}$.

Now, motivated also by the notions given in [7] and [5], we set the following definition of the Lefschetz class of a Lie $\Gamma$-groupoid, as localization of the equivariant index.

Definition 2.14. Let $G$ be a Lie $\Gamma$-groupoid, let $P \in \Psi_{c}^{m}(G, E)$ be a $\Gamma$-invariant elliptic pseudodifferential $G$-operator and let $h \in \Gamma$.

1. The smooth Lefschetz class $L_{\infty}([h], P)$ of the conjugacy class $[h]$ of $h$ relative to $P$ is the smooth equivariant analytic index class $i n d_{C^{\infty}}^{\Gamma}(P)$ of $P$ localized at $I_{[h]}$. In symbols

$$
L_{\infty}([h], P)=\frac{i n d_{C^{\infty}}^{\Gamma}(P)}{1_{R[\Gamma]}} \in K_{0}^{\Gamma}\left(C_{c}^{\infty}(G, E)\right)_{[h]} .
$$

2. The $C^{*}$-algebraic Lefschetz class $L([h], P)$ of $[h]$ relative to $P$ is the localized $C^{*}$-algebraic equivariant index class with respect to $I_{[h]}$,

$$
L([h], P)=\frac{i n d_{a}^{\Gamma}(P)}{1_{R[\Gamma]}} \in K_{0}^{\Gamma}\left(C_{r}^{*}(G, E)\right)_{[h]} .
$$

Remark 2.5. If $\Gamma^{\prime}$ is a compact abelian Lie group, then the conjugacy class $[h]$ of any element $h \in \Gamma^{\prime}$ contains only the element $h$ itself. In this case, we define the smooth Lefschetz class $L_{\infty}(h, P)$ of the diffeomorphism $h$ relative to the $\Gamma^{\prime}$-invariant pseudodifferential operator $P$ as

$$
L_{\infty}(h, P)=\frac{i n d_{C^{\infty}}^{\Gamma^{\prime}}(P)}{1_{R\left[\Gamma^{\prime}\right]}} \in K_{0}^{\Gamma^{\prime}}\left(C_{c}^{\infty}(G, E)\right)_{h},
$$


where, by abuse of notation, we have denoted $K_{0}^{\Gamma^{\prime}}\left(C_{c}^{\infty}(G, E)\right)_{[h]}$ simply by $K_{0}^{\Gamma^{\prime}}\left(C_{c}^{\infty}(G, E)\right)_{h}$.

Analogously, we define the $C^{*}$-algebraic Lefschetz class $L(h, P)$ of the element $h$ relative to $P$ as the localized $C^{*}$-algebraic equivariant index class with respect to $\Gamma^{\prime}$.

Our aim now is focusing on the particular case of a foliated $\Gamma$-manifold and studying the Lefschetz classes introduced above for the holonomy groupoid and the twisted holonomy groupoid of the foliation.

Let $(M, \mathcal{F}, \Gamma)$ be a foliated $\Gamma$-manifold and $E$ be a Hermitian vector bundle on $M$. Let $H(\mathcal{F}, \Gamma)$ denote its twisted holonomy groupoid and $H(\mathcal{F}) \subseteq H(\mathcal{F}, \Gamma)$ the holonomy groupoid.

For notational simplicity and for the rest of this section, we will denote the bundle of half densities $\Omega^{1 / 2} H(\mathcal{F}, \Gamma)$ over $H(\mathcal{F}, \Gamma)$ and $\Omega^{1 / 2} H(\mathcal{F})$ over $H(\mathcal{F})$ by the same symbol $\Omega^{1 / 2}$, since it will be clear from the context which bundle is meant.

Finally let us assume the group $\Gamma$ to be a compact Lie group.

The $\Gamma$-equivariant algebra homomorphism

$$
\left.j: C_{c}^{\infty}\left(H(\mathcal{F}), E N D(E) \otimes \Omega^{1 / 2}\right) \hookrightarrow C_{c}^{\infty}\left(H(\mathcal{F}, \Gamma), E N D(E) \otimes \Omega^{1 / 2}\right)\right)
$$

induces a corresponding $R[\Gamma]$-linear map

$j_{*}: K_{0}^{\Gamma}\left(C_{c}^{\infty}\left(H(\mathcal{F}), E N D(E) \otimes \Omega^{1 / 2}\right)\right) \rightarrow K_{0}^{\Gamma}\left(C_{c}^{\infty}\left(H(\mathcal{F}, \Gamma), E N D(E) \otimes \Omega^{1 / 2}\right)\right)$.

Since this map is $R[\Gamma]$-linear, picking $h \in \Gamma$, we get by functoriality the localized $R[\Gamma]_{[[h]}$-linear maps

$j_{[h]}: K_{0}^{\Gamma}\left(C_{c}^{\infty}\left(H(\mathcal{F}), E N D(E) \otimes \Omega^{1 / 2}\right)\right)_{[h]} \rightarrow K_{0}^{\Gamma}\left(C_{c}^{\infty}\left(H(\mathcal{F}, \Gamma), E N D(E) \otimes \Omega^{1 / 2}\right)\right)_{[h]}$.

Now, let $P \in \Psi_{c}^{m}(H(\mathcal{F}, \Gamma) ; E)$ be $\Gamma$-invariant and elliptic and let $h \in \Gamma$. Then, the smooth equivariant index class is an equivariant $K$-theory class $\operatorname{ind}_{C^{\infty}}^{\Gamma}(P) \in K_{0}^{\Gamma}\left(C_{c}^{\infty}\left(H(\mathcal{F}, \Gamma), E N D(E) \otimes \Omega^{1 / 2}\right)\right)$ and the Lefschetz class of $[h]$ relative to $P$ is the corresponding localized class

$$
L_{\infty}([h], P)=\frac{i n d_{C^{\infty}}^{\Gamma}(P)}{1_{R[\Gamma]}} \in K_{0}^{\Gamma}\left(C_{c}^{\infty}\left(H(\mathcal{F}, \Gamma), E N D(E) \otimes \Omega^{1 / 2}\right)\right)_{[h]} .
$$

Analogously, one gets the $C^{*}$-algebraic equivariant index and the $C^{*}$-algebraic Lefschetz classes.

In the same way, if $D \in \Psi_{c}^{m}(H(\mathcal{F}) ; E)$ is instead a $\Gamma$-invariant elliptic pseudodifferential operator on the holonomy groupoid $H(\mathcal{F}) \subseteq H(\mathcal{F}, \Gamma)$, then its smooth equivariant index class $i n d_{C^{\infty}}^{\Gamma}(D)$ is a class in $K_{0}^{\Gamma}\left(C_{c}^{\infty}\left(H(\mathcal{F}), E N D(E) \otimes \Omega^{1 / 2}\right)\right)$ and the corresponding smooth Lefschetz class on $H(\mathcal{F})$ of $[h]$ will be denoted by

$$
\mathcal{L}_{\infty}([h], D)=\frac{i n d_{C_{\infty}}^{\Gamma}(D)}{1_{R[\Gamma]}} \in K_{0}^{\Gamma}\left(C_{c}^{\infty}\left(H(\mathcal{F}), E N D(E) \otimes \Omega^{1 / 2}\right)\right)_{[h]} .
$$

Notice that we have two ways to think of the index of $D$ as being an element of $K_{0}^{\Gamma}\left(C_{c}^{\infty}\left(H(\mathcal{F}, \Gamma), E N D(E) \otimes \Omega^{1 / 2}\right)\right)$ : 
- we can extend $D$ to the operator $j(D) \in \Psi_{c}^{m}(H(\mathcal{F}, \Gamma) ; E)$ by means of Equation 2.47 and take the index of $j(D)$;

- we can take $\operatorname{ind}_{C^{\infty}}^{\Gamma}(D) \in K_{0}^{\Gamma}\left(C_{c}^{\infty}\left(H(\mathcal{F}), E N D(E) \otimes \Omega^{1 / 2}\right)\right)$ and map this class through the map $j_{*}$.

The following proposition ensures that these two ways are equivalent.

Proposition 2.2.9. If $D \in \Psi_{c}^{m}(H(\mathcal{F}) ; E)$ is $\Gamma$-invariant and elliptic and $j(D) \in \Psi_{c}^{m}(H(\mathcal{F}, \Gamma) ; E)$ is the corresponding operator on $H(\mathcal{F}, \Gamma)$, then the following equalities hold

$$
j_{*}\left(i n d_{C^{\infty}}^{\Gamma}(D)\right)=i n d_{C^{\infty}}^{\Gamma}(j(D)) \in K_{0}^{\Gamma}\left(C_{c}^{\infty}\left(H(\mathcal{F}, \Gamma), E N D(E) \otimes \Omega^{1 / 2}\right)\right)
$$

and

$$
j_{[h]}\left(L_{\infty}([h], D)\right)=L_{\infty}([h], j(D)) \in K_{0}^{\Gamma}\left(C_{c}^{\infty}\left(H(\mathcal{F}, \Gamma), E N D(E) \otimes \Omega^{1 / 2}\right)\right)_{[h]}
$$

Proof. Since $P$ is $\Gamma$-invariant and elliptic, there exists a $\Gamma$-invariant parametrix $Q$ for $P$ such that $S_{0}=1-Q P, S_{1}=1-P Q \in \Psi_{c}^{-\infty}(H(\mathcal{F}) ; E)$. Moreover, since the inclusion $j: \Psi_{c}^{\infty}(H(\mathcal{F}) ; E) \hookrightarrow \Psi_{c}^{\infty}(H(\mathcal{F}, \Gamma) ; E)$ is an equivariant unital algebra homomorphism, it follows that $j(Q)$ is a $\Gamma$-invariant parametrix for $j(P)$, with remainders $j\left(S_{0}\right)$ and $j\left(S_{1}\right)$.

The smooth equivariant index class of $P$ is, by definition, the formal difference of the equivalence classes of the idempotents $e=\left(\begin{array}{ll}1 & 0 \\ 0 & 0\end{array}\right)$ and $T e T^{-1}=$ $\left(\begin{array}{cc}1-S_{1}^{2} & \left(S_{1}+S_{1}^{2}\right) P \\ S_{0} Q & S_{0}^{2}\end{array}\right)$.

In the same way, the smooth equivariant index of $j(P)$ is the formal difference of $j(e)=e$ and the idempotent $R=\left(\begin{array}{cc}1-j\left(S_{1}\right)^{2} & \left(j\left(S_{1}\right)+j\left(S_{1}\right)^{2}\right) j(P) \\ j\left(S_{0}\right) j(Q) & j\left(S_{0}\right)^{2}\end{array}\right)$. The particular choice of the parametrix $j(Q)$ to construct the index class of $j(P)$ is irrelevant, since the index does not depend on the parametrix.

Now, with these choices one gets that $j\left(T e T^{-1}\right)=R$ and thus

$j_{*}\left(i n d_{C^{\infty}}^{\Gamma}(P)\right)=j_{*}\left(\left[T e T^{-1}\right]-[e]\right)=\left[j\left(T e T^{-1}\right)\right]-[e]=[R]-[e]=i n d_{C^{\infty}}^{\Gamma}(j(P))$

which proves the first equality.

The second one follows from the equality of the index classes and from the functoriality of the localization procedure.

As already mentioned, the equivariant index class of an elliptic operator and the corresponding Lefschetz class of the conjugacy class of a diffeomorphism $h \in \Gamma$ are complicated invariants of the foliated $\Gamma$-manifold $(M, \mathcal{F}, \Gamma)$ and the operator. In particular, the non-vanishing of the Lefschetz class is a difficult problem to solve in general. One strategy to attack this problem, or, more generally, to investigate these classes is to construct additive maps defined on $K_{0}^{\Gamma}\left(C_{c}^{\infty}\left(H(\mathcal{F}, \Gamma), E N D(E) \otimes \Omega^{1 / 2}\right)\right)_{[h]}$ or $K_{0}^{\Gamma}\left(C_{c}^{\infty}\left(H(\mathcal{F}), E N D(E) \otimes \Omega^{1 / 2}\right)\right)_{[h]}$ 
to $\mathbb{C}$. These maps should reflect topological/geometric and dynamical features of the foliation. Thus the evaluation of such a map on the Lefschetz class should provide invariants of the foliation.

These maps arise from suitable equivariant cyclic cocycles, our object of study in the next chapters.

\subsection{Pseudo-differential calculus for foliated bun- dles}

In the particular case of a foliated bundle $\left(\tilde{M} \times_{H} T, \mathcal{F}, \pi\right)$, the pseudodifferential calculus introduced in the previous section, can be slightly simplified. Indeed, instead of using families on the holonomy groupoid, one may use smooth $H$-invariant families of classical pseudodifferential operators on the manifolds $\tilde{M}_{x}$, for all points $x \in T$. The resulting pseudodifferential calculus is equivalent to that defined using the holonomy groupoid, in the sense that there exists a bijection between these two spaces of operators. However for many purposes, it is technically simpler to deal with.

Let us give in this section a brief overview of this calculus. We will only sketch the proofs, giving precise references in which those can be found. For a more detailed treatment we refer to [41] and 8].

Let $\left(\tilde{M} \times_{H} T, \mathcal{F}, \pi\right)$ be a foliated bundle, as defined in Example 1.3. Assume that the action of $H$ on $T$ is locally free, namely given $g \in H$, if there exists an open set $U \subseteq T$ such that $x g=x$, for all $x \in U$, then $g=e$, the identity of $H$.

Under this assumption, it follows that there exists a Lie groupoid isomorphism between the holonomy groupoid $H(\mathcal{F})$ of the foliated bundle and its monodromy groupoid $\Pi(\mathcal{F})=(\tilde{M} \times \tilde{M} \times T) / H$. In particular, $H(\mathcal{F})$ is Hausdorff. Through this section we will always assume the $H$-action on $T$ to be locally free (for comments, see [41] or [12]).

Let $\tilde{E}$ and $\tilde{F}$ be two $H$-equivariant Hermitian vector bundles on $\tilde{M} \times T$. Further denote by $C_{c}^{\infty}(\tilde{M} \times T, \tilde{E})$ the space of compactly supported smooth sections of $\tilde{E}$ over $\tilde{M} \times T$.

Definition 2.15. Let $P: C_{c}^{\infty}(\tilde{M} \times T, \tilde{E}) \rightarrow C^{\infty}(\tilde{M} \times T, \tilde{F})$ a linear operator. We say that $P$ is a $H$-invariant family of pseudodifferential operators of order $m \in \mathbb{Z}$ if

1. for each $t \in T$, the operator $P$ restricts on $\tilde{M}_{t}=p r_{2}^{-1}(t)$ to an operator

$$
P_{t}: C_{c}^{\infty}\left(\tilde{M}_{t}, \tilde{E}_{t}\right) \rightarrow C^{\infty}\left(\tilde{M}_{t}, \tilde{F}_{t}\right)
$$

which is a classical pseudodifferential operator of order $m$. Here $\tilde{E}_{t}=\tilde{E}_{\mid M_{t}}$, We require further that the family of pseudodifferential operators $\left(P_{t}\right)_{t \in T}$ is smooth with respect to $t \in T$, as defined in [42].

2. $P$ is $H$-equivariant, i.e. $g P_{x} g^{-1}=P_{g x}$, for any $x \in T, g \in H$.

3. the distributional kernel of $P$ has $H$-compact support. 
Conditions (1) and (2) imply that the distributional kernel can be thought of as a distribution on $\tilde{M} \times \tilde{M} \times T$ which is $H$-invariant. Therefore it can be regarded as a distribution on the holonomy groupoid $H(\mathcal{F})$. Condition (3) means that this distribution has compact support and therefore the operator $P$ maps $C_{c}^{\infty}(\tilde{M} \times T ; \tilde{E})$ to $C_{c}^{\infty}(\tilde{M} \times T ; \tilde{F})$.

Let $m \in \mathbb{Z}$ and denote the space of $H$-invariant families of pseudodifferential operators of order $\leq m$ from $\tilde{E}$ to $\tilde{F}$ by $\Psi_{H}^{m}(\tilde{E}, \tilde{F})$. A very important fact is that if $P \in \Psi_{H}^{m}\left(\tilde{E}^{0}, \tilde{E}^{1}\right)$ and $Q \in \Psi_{H}^{k}\left(\tilde{E}^{1}, \tilde{E}^{2}\right.$, then the composition $Q P \in$ $\Psi_{H}^{m+k}\left(\tilde{E}^{0}, \tilde{E}^{2}\right)$. Set

$$
\Psi_{H}^{\infty}(\tilde{E}, \tilde{F})=\bigcup_{m \in \mathbb{Z}} \Psi_{H}^{m}(\tilde{E}, \tilde{F})
$$

In the case $\tilde{E}=\tilde{F}$ we obtain the filtered algebra $\Psi_{H}^{\infty}(\tilde{E}, \tilde{E})$, simply denoted by $\Psi_{H}^{\infty}(\tilde{E})$. Moreover if $P \in \Psi_{H}^{m}(\tilde{E}, \tilde{F})$ we can associate the formal adjoint $P^{*}=\left(P_{t}^{*}\right)_{t \in T}$, which is an element in $\Psi_{H}^{m}(\tilde{F}, \tilde{E})$. This is an involution in the case $\tilde{E}=\tilde{F}$, giving $\Psi_{H}^{\infty}(\tilde{E})$ the structure of a $*$-algebra.

Important subalgebras of $\Psi_{H}^{\infty}(\tilde{E})$ are the algebras $\Psi_{H}^{0}(\tilde{E})$ of pseudodifferential operators of order $\leq 0$ and $\Psi_{H}^{-\infty}(\tilde{E})$ of pseudodifferential operators of order $-\infty$. The latter are also called compactly smoothing operators.

Remark 2.6. Let $E$ denote a vector bundle over $\tilde{M} \times_{H} T$ and $\tilde{E}$ its pullback over $\tilde{M} \times T$.

There exists an isomorphism between the algebra of pseudodifferential operators $\Psi_{\tilde{H}}^{\infty}(\tilde{E})$, as defined in 2.15 and the algebra $\Psi_{c}^{\infty}(H(\mathcal{F}), E)$, where $H(\mathcal{F})=(\tilde{M} \times$ $\tilde{M} \times T) / H$.

For the definition of this isomorphism we refer to [8], Section 3.2.

Thus in the case of foliated bundles, instead of using the general pseudodifferential calculus we can alternatively use $H$-invariant families on the transversal $T$. This is technically simpler.

Let $\pi_{\text {vert }}: T_{\text {vert }}^{*}(\tilde{M} \times T) \rightarrow \tilde{M} \times T$ be the vertical cotangent bundle of the product fibration $\tilde{M} \times T$ and consider the vector bundle $\operatorname{Hom}\left(\pi_{\text {vert }}^{*} \tilde{E}, \pi_{\text {vert }}^{*} \tilde{F}\right)$ on $T_{\text {vert }}^{*}(\tilde{M} \times T)$. Since the projection $\pi_{\text {vert }}$ is $H$-equivariant, then the Hombundle above carries an induced $H$-action.

If $P \in \Psi_{H}^{m}(\tilde{E}, \tilde{F})$, its principal symbol is a well defined $H$-invariant section $\sigma_{m}(P) \in C^{\infty}\left(T_{\text {vert }}^{*}(\tilde{M} \times T) ; \operatorname{Hom}\left(\pi_{\text {vert }}^{*} \tilde{E}, \pi_{\text {vert }}^{*} \tilde{F}\right)\right)$. Equivalently, $\sigma_{m}(P)$ is a well defined section on the cotangent bundle to the foliation $T^{*} \mathcal{F}$ with values in $\operatorname{Hom}\left(\pi_{\mathcal{F}}^{*}(E), \pi_{\mathcal{F}}^{*}(F)\right)$. Choosing an Euclidean inner product on $T^{*} \mathcal{F}$, we see that $\sigma_{m}(P)$ is completely determined by its restriction to the cosphere bundle $S^{*} \mathcal{F}$ of the foliation, since the operators we deal with are classical. Therefore one has that $\sigma_{m}(P) \in C^{\infty}\left(S^{*} \mathcal{F} ; \operatorname{Hom}\left(\pi_{\mathcal{F}}^{*}(E), \pi_{\mathcal{F}}^{*}(F)\right)\right)$.

More generally, one can introduce the principal symbol map

$$
\sigma_{m}: \Psi_{H}^{m}(\tilde{E}, \tilde{F}) \rightarrow C^{\infty}\left(S^{*} \mathcal{F} ; \operatorname{Hom}\left(\pi_{\mathcal{F}}^{*}(E), \pi_{\mathcal{F}}^{*} F\right)\right)
$$

Definition 2.16. Let $P \in \Psi_{H}^{m}(\tilde{E}, \tilde{F})$. We say that $P$ is elliptic if its principal symbol $\sigma_{m}(P)$ is invertible, for each $(x, \xi) \in S^{*} \mathcal{F}$. 
The following result is the analogue of 2.2 .5 in the context of foliated bundles.

Theorem 2.3.1. If $P \in \Psi_{H}^{l}(\tilde{E}, \tilde{F})$ is elliptic, then there exists an operator $Q \in \Psi_{H}^{-l}(\tilde{F}, \tilde{E})$ such that

$$
I d-P Q=R_{0} \in \Psi_{H}^{-\infty}(\tilde{F}, \tilde{F}), \quad I d-Q P=R_{1} \in \Psi_{H}^{-\infty}(\tilde{E}, \tilde{E})
$$

The operator $Q$ is called a parametrix for $P$.

Notice now that a pseudodifferential operator $P \in \Psi_{H}^{0}(\tilde{E})$ of order $\leq 0$ extends to a continuous family of bounded operators for the field of Hilbert spaces $L_{\tau}^{2}(\tilde{E})$. We define the norm

$$
\|P\|=\sup _{t \in T}\left\|P_{t}\right\|
$$

for every $P \in \Psi_{H}^{0}(\tilde{E})$ and take the closure $\overline{\Psi_{H}^{0}(\tilde{E})}\|\cdot\|$ with respect to this norm.

Theorem 2.3.2. The principal symbol map extends to the surjective linear map

$$
\sigma_{0}: \overline{\Psi_{H}^{0}(\tilde{E})} \rightarrow C\left(S^{*} \mathcal{F}, \operatorname{Hom}\left(\pi_{\mathcal{F}}^{*}(E), \pi_{\mathcal{F}}^{*}(F)\right)\right.
$$

and we have the following exact sequence of $C^{*}$-algebras

$$
0 \rightarrow C^{*}\left(\tilde{M} \times_{H} T, \mathcal{F} ; E\right) \rightarrow \overline{\Psi_{H}^{0}(\tilde{E})} \stackrel{\sigma_{0}}{\longrightarrow} C\left(S^{*} \mathcal{F}, \operatorname{Hom}\left(\pi_{\mathcal{F}}^{*}(E), \pi_{\mathcal{F}}^{*}(F)\right) \rightarrow 0\right.
$$

Let us now suppose that a Lie group $\Gamma$ acts on $\left(\tilde{M} \times_{H} T, \mathcal{F}, \pi\right)$ by leafpreserving diffeomorphisms, making it into a foliated $\Gamma$-manifold. Then, all the results about the induced action of $\Gamma$ on the algebra of pseudodifferential operators $\Psi_{H}^{\infty}(\tilde{E})$ are exactly the same as those stated in Section 2.2.2. Therefore we do not repeat them here, referring to Section 2.2 .2 and the references therein for the details. 


\section{Chapter 3}

\section{Equivariant and Twisted Cyclic Cohomology}

In this chapter we present the definitions of the equivariant Hochschild, equivariant cyclic and equivariant periodic cyclic cohomology groups of a certain class of locally convex $\mathbb{C}$-algebras acted upon by a compact Lie group $\Gamma$, and establish the basic properties of these functors. In particular, we will prove an equivariant SBI-sequence, that relates equivariant Hochschild cohomology and equivariant cyclic cohomology.

Furthermore, we will study the pairing between equivariant $K_{0}$-theory and the equivariant cyclic cocycles on $(\mathcal{A}, \Gamma)$. This pairing is fundamental for higher index theory, both equivariant and nonequivariant. The nonequivariant case has been extensively studied in various geometric situations like discrete group actions on manifolds and foliations, giving rise to deep and unexpected results (cf. [17] and [16] and the references therein for details and further remarks). Instead, the equivariant situation has not yet been fully explored and it is interesting to investigate further in this direction.

Our treatment is based on [37, [18, 7] and [46].

\subsection{Equivariant Cyclic Cohomology for unital $\Gamma-$ algebras}

In this section we introduce the basic objects and operators we will deal with in the rest of the chapter, namely equivariant (Hochschild) cochains, the equivariant Hochschild differential maps $b_{\Gamma}$ and $b_{\Gamma}^{\prime}$ and the equivariant cyclic operators $\lambda_{\Gamma}$ on cochains.

Unlike the nonequivariant case, the definitions of the differential $b_{\Gamma}$ and of the cyclic operator must be suitably modified, to keep track of the $\Gamma$-action. Doing this, one gets again that the operator $b_{\Gamma}$ is well-defined on the $\lambda_{\Gamma}$-invariant cochains, giving rise to a cochain complex $\left(C_{\lambda_{\Gamma}}^{*}(\mathcal{A}, \Gamma), b_{\Gamma}\right)$, whose homology is by definition the equivariant cyclic cohomology of the $\Gamma$-algebra $\mathcal{A}$. 
We shall not attempt to deal with general locally convex algebras, but rather we will treat for simplicity a special class of locally convex algebras, namely Fréchet algebras, in order to emphasize the algebraic aspects of the equivariant cyclic theory. If the group happens to be finite, it is not necessary to consider a topology and the theory is purely algebraic.

Finally, we will first assume that the algebras are unital. We shall see in the next sections how to get rid of this assumption.

If $\mathcal{A}$ is a unital locally convex $\mathbb{C}$-algebra acted upon by a compact Lie group $\Gamma$, recall that $\mathcal{A}$ is a $\Gamma$-algebra if there is a group homomorphism

$$
\rho: \Gamma \rightarrow \operatorname{Aut}(\mathcal{A})
$$

where $\operatorname{Aut}(\mathcal{A})$ is the group of continuous automorphisms of $\mathcal{A}$, which is strongly continuous, i.e. for any $a \in \mathcal{A}$ the map

$$
g \rightarrow \rho_{g}(a)
$$

is continuous with respect to the topology on $\mathcal{A}$. As usual, we will often forget to mention explicitly the homomorphism $\rho$, if there is no cause of confusion.

Now, let us consider a unital Fréchet $\Gamma$-algebra $\mathcal{A}$ and let $C(\Gamma)$ denote the $C^{*}$-algebra of complex valued continuous functions on $\Gamma$. Denote by $\mathcal{L}^{n}(\mathcal{A}, C(\Gamma))$ the vector space of continuous $(n+1)$-linear maps $\tau: \mathcal{A} \times \cdots \times \mathcal{A} \rightarrow C(\Gamma)$. The action of $\Gamma$ on $\mathcal{A}$ and on $C(\Gamma)$, given by the conjugation in the argument, induces a natural action on this space, namely for any $g, h \in \Gamma, a_{i} \in \mathcal{A}, \tau \in \mathcal{L}^{n}(\mathcal{A}, C(\Gamma))$

$$
(g \cdot \tau)\left(a_{0}, a_{1}, \ldots, a_{n}\right)(h):=\tau\left(g a_{0}, g a_{1}, \ldots, g a_{n}\right)\left(g h g^{-1}\right)
$$

Definition 3.1. A map $\tau \in \mathcal{L}^{n}(\mathcal{A}, C(\Gamma))$ is called $\Gamma$-invariant if for all $g \in \Gamma$

$$
g \cdot \tau=\tau
$$

The vector space of all $\Gamma$-invariant mappings $\tau \in \mathcal{L}^{n}(\mathcal{A}, C(\Gamma))$ will be denoted by $C^{n}(\mathcal{A}, \Gamma)$ and an element of it will be called equivariant Hochschild cochain, or simply equivariant cochain.

Following [7] we will adopt the notation $\tau\left(a_{0}, a_{1}, \ldots, a_{n} \mid h\right)$ for the scalar $\tau\left(a_{0}, a_{1}, \ldots, a_{n}\right)(h)$, for all elements $a_{i} \in \mathcal{A}, h \in \Gamma$.

The corresponding graded vector space is denoted by

$$
C^{*}(\mathcal{A}, \Gamma)=\bigoplus_{n \geq 0} C^{n}(\mathcal{A}, \Gamma)
$$

Remark 3.1. If the group $\Gamma$ is a finite and $\mathcal{A}$ is a (nontopological) $\Gamma$-algebra, one can consider the vector space $\mathcal{L}^{n}(\mathcal{A}, F(\Gamma))$ of $(n+1)$-linear maps on $\mathcal{A}$ with values in $F(\Gamma)$, the space of all complex valued functions on $\Gamma$. Again, the action of $\Gamma$ on $\mathcal{A}$ and on $F(\Gamma)$ by conjugation determines an action on $\mathcal{L}^{n}(\mathcal{A}, F(\Gamma))$ and we take the $\Gamma$-invariant elements of $\mathcal{L}^{n}(\mathcal{A}, F(\Gamma))$. The subspace of invariant maps is denoted as above $C^{n}(\mathcal{A}, \Gamma)$. 


\subsubsection{Cyclic group action}

Let $\Gamma$ be a compact Lie group and let $\mathcal{A}$ be a unital Fréchet $\Gamma$-algebra. Like in the nonequivariant theory, one notes that on $C^{n}(\mathcal{A}, \Gamma)$ an action of the cyclic group $\mathbb{Z} /(n+1) \mathbb{Z}$ is naturally defined. However, in the equivariant case, the representation of $\mathbb{Z} /(n+1) \mathbb{Z}$ on the space of equivariant Hochschild cochains $C^{n}(\mathcal{A}, G)$ is provided by a cyclic operator which takes into account the presence of the group $\Gamma$. More precisely

Definition 3.2. The operator $\lambda_{\Gamma}: C^{n}(\mathcal{A}, \Gamma) \rightarrow C^{n}(\mathcal{A}, \Gamma)$ acts on $\tau \in C^{n}(\mathcal{A}, \Gamma)$ by

$$
\left(\lambda_{\Gamma} \tau\right)\left(a_{0}, a_{1}, \ldots, a_{n} \mid g\right):=(-1)^{n} \tau\left(g^{-1}\left(a_{n}\right), a_{0}, \ldots, a_{n-1} \mid g\right)
$$

This is the equivariant cyclic operator on $C^{n}(\mathcal{A}, \Gamma)$.

Remark 3.2. The equivariant cyclic operator $\lambda_{\Gamma}$ induces a representation of the cyclic group $\mathbb{Z} /(n+1) \mathbb{Z}$ on the equivariant cochains. The only thing to prove, which is not evident is the relation

$$
\lambda_{\Gamma}^{n+1}=i d
$$

One gets

$$
\begin{array}{r}
\lambda_{\Gamma}^{n+1} \tau\left(a_{0}, a_{1}, \ldots, a_{n} \mid g\right)=(-1)^{n} \lambda_{\Gamma}^{n} \tau\left(g^{-1} a_{n}, a_{0}, \ldots, a_{n-1} \mid g\right)= \\
=\cdots=(-1)^{n(n+1)} \tau\left(g^{-1} a_{0}, g^{-1} a_{1}, \ldots, g^{-1} a_{n} \mid g\right)=\tau\left(a_{0}, a_{1}, \ldots, a_{n} \mid g\right)
\end{array}
$$

The last equality follows from the invariance of the cochain. Therefore 3.4 is proved.

Definition 3.3. The norm operator is defined as

$$
N_{\Gamma}:=\sum_{i=0}^{n} \lambda_{\Gamma}^{i}
$$

To simplify notations and formulas in what follows, we will often write $\lambda$ instead of $\lambda_{\Gamma}$ and analogously $N$ in place of $N_{\Gamma}$.

Now by 3.4 it is obvious that the following relation holds

$$
(i d-\lambda) N=N(i d-\lambda)=0 .
$$

This relation and the following lemma are going to play an important role for the definition of equivariant cyclic cohomology.

Lemma 3.1.1. For any $n \in \mathbb{N}$

$$
C^{n}(\mathcal{A}, \Gamma) \stackrel{i d-\lambda}{\longrightarrow} C^{n}(\mathcal{A}, \Gamma) \stackrel{N}{\longrightarrow} C^{n}(\mathcal{A}, \Gamma) \stackrel{i d-\lambda}{\longrightarrow} C^{n}(\mathcal{A}, \Gamma) \stackrel{N}{\longrightarrow} \ldots
$$

is a $\mathbb{Z} /(n+1) \mathbb{Z}$-complex.

Moreover this complex is acyclic. 
Proof. By Equation 3.6 it is clear that 3.7 is a complex. Since we are working with algebras on a field of characteristic 0 , namely with $\mathbb{C}$-algebras, there is a homotopy from the identity chain map to the zero chain map of 3.7 , like in the nonequivariant theory. Set

$$
G^{\prime}:=1 /(n+1) \cdot i d \quad G:=-\frac{1}{n+1} \sum_{j=0}^{n} j \lambda^{j} .
$$

One verifies that

$$
\begin{aligned}
& G^{\prime} N+(1-\lambda) G=i d \\
& N G^{\prime}+G(1-\lambda)=i d
\end{aligned}
$$

Therefore $\left(G, G^{\prime}\right)$ is a chain homotopy from id to 0 and the complex is acyclic.

Remark 3.3. If we consider algebras over arbitrary fields or even rings (the theory for such general algebras in the nonequivariant case has been extensively studied. See [37]), it is no longer true that the complex 3.7 is acyclic.

\subsubsection{Equivariant Hochschild cohomology}

In this subsection we will define equivariant Hochschild cohomology for a unital Fréchet $\Gamma$-algebra with $\Gamma$ a compact Lie group. The assumption of the unitality of the algebra is essential and in the non-unital case the definition must be modified. We will see how to modify it in the next section. As for the equivariant cyclic operator $\lambda_{\Gamma}$, the Hochschild differential $b_{\Gamma}$ is suitably defined to keep track of the action of $\Gamma$.

Our treatment is based on the approach developed in [7] and [34].

Let $\Gamma$ be a compact Lie group and let $\mathcal{A}$ be a unital Fréchet $\Gamma$-algebra.

Definition 3.4. Let $\tau \in C^{n}(\mathcal{A}, \Gamma)$. The operator $b_{\Gamma}^{\prime}$ on $C^{n}(\mathcal{A}, \Gamma)$ is defined by

$$
\left(b_{\Gamma}^{\prime} \tau\right)\left(a_{0}, a_{1}, \ldots, a_{n+1} \mid g\right)=\sum_{i=0}^{n}(-1)^{i} \tau\left(a_{0}, a_{1}, \ldots, a_{i} a_{i+1}, \ldots, a_{n+1} \mid g\right)
$$

It is easy to verify that $b_{\Gamma}^{\prime} \tau \in C^{n+1}(\mathcal{A}, \Gamma)$, for any $\tau \in C^{n}(\mathcal{A}, \Gamma)$.

Since the definition of the operator $b_{\Gamma}^{\prime}$ is the same as in the nonequivariant theory, we deduce that $\left(b_{\Gamma}^{\prime}\right)^{2}=0$, i.e. it is indeed a differential for the graded vector space $C^{*}(\mathcal{A}, \Gamma)$, and, further, since we are also assuming the algebras to be unital, the resulting complex $\left(C^{*}(\mathcal{A}, \Gamma), b_{\Gamma}^{\prime}\right)$ is acyclic.

In fact the operators $s: C^{n}(\mathcal{A}, \Gamma) \rightarrow C^{n-1}(\mathcal{A}, \Gamma)$ given by

$$
(s \tau)\left(a_{0}, a_{1}, \ldots, a_{n-1} \mid g\right)=(-1)^{n-1} \tau\left(a_{0}, a_{1}, \ldots, a_{n-1}, 1 \mid g\right)
$$

are chain contractions for $\left(C^{*}(\mathcal{A}, \Gamma), b_{\Gamma}^{\prime}\right)$.

Set, now, for any $\tau \in C^{n}(\mathcal{A}, \Gamma)$

$$
\left(d_{\Gamma} \tau\right)\left(a_{0}, \ldots, a_{n+1} \mid g\right)=(-1)^{n+1} \tau\left(g^{-1}\left(a_{n+1}\right) a_{0}, \ldots, a_{n} \mid g\right)
$$


Definition 3.5. The equivariant Hochschild coboundary map $b_{\Gamma}: C^{n}(\mathcal{A}, \Gamma) \rightarrow$ $C^{n+1}(\mathcal{A}, \Gamma)$ is the operator given by

$$
b_{\Gamma}=b_{\Gamma}^{\prime}+d_{\Gamma}
$$

It is obvious that $C^{*}(\mathcal{A}, \Gamma)$ is invariant for $b_{\Gamma}$. This operator is the equivariant version of the Hochschild coboundary operator in the nonequivariant theory. Indeed

Lemma 3.1.2. $b_{\Gamma}^{2}=0$

Proof. From the definition it follows that

$$
b_{\Gamma}^{2}=\left(b_{\Gamma}^{\prime}+d_{\Gamma}\right)^{2}=\left(b_{\Gamma}^{\prime}\right)^{2}+b_{\Gamma}^{\prime} d_{\Gamma}+d_{\Gamma} b_{\Gamma}^{\prime}+d_{\Gamma}^{2} .
$$

Now, we already know that $\left(b_{\Gamma}^{\prime}\right)^{2}=0$. Moreover, by a direct computation, one can show that $b_{\Gamma}^{\prime} d_{\Gamma}+d_{\Gamma} b_{\Gamma}^{\prime}+d_{\Gamma}^{2}=0$, from which the result follows.

Now we are in the position to define the equivariant Hochschild complex for $(\mathcal{A}, \Gamma)$. We remark again that the unitality assumption is essential here.

Definition 3.6. The equivariant Hochschild complex is the cochain complex $\left(C^{*}(\mathcal{A}, \Gamma), b_{\Gamma}\right)$. The equivariant Hochschild cohomology of $(\mathcal{A}, \Gamma)$ is the homology of this complex, i.e.

$$
H H^{n}(\mathcal{A}, \Gamma)=H_{n}\left(C^{*}(\mathcal{A}, \Gamma), b_{\Gamma}\right)
$$

This is the correct definition of equivariant Hochschild cohomology only in the unital case. If the algebra $\mathcal{A}$ has not got a unit we need an alternative approach to define this theory and we will see that in general the cyclic operator $\lambda$ and the complex $\left(C^{*}(\mathcal{A}, \Gamma), b_{\Gamma}^{\prime}\right)$ must be used to give the correct notion of equivariant Hochschild cohomology. These aspects will be discussed at lenght in the next section.

Now, note that, if the group $G$ is the trivial group consisting only of the identity element $\Gamma=e$, then one clearly recovers the nonequivariant Hochschild cohomology groups of the algebra $\mathcal{A}$.

Remark 3.4. For a finite group $\Gamma$ and a $\Gamma$-algebra $\mathcal{A}$ we can define the equivariant Hochschild complex and equivariant Hochschild cohomology in exactly the same way.

\subsubsection{Equivariant cyclic cohomology}

In this subsection we will define equivariant cyclic cohomology directly from the equivariant Hochschild complex $\left(C^{*}(\mathcal{A}, \Gamma), b_{\Gamma}\right)$. The following definition is inspired by the original one of $\mathrm{A}$. Connes, in the nonequivariant theory, and can be found in the paper $[7$.

Let $\mathcal{A}$ be as above a unital Fréchet $\Gamma$-algebra. The main idea to define equivariant cyclic cohomology is the following. Once one has the cyclic action of $\mathbb{Z} /(n+1) \mathbb{Z}$ on $C^{n}(\mathcal{A}, \Gamma)$, important cochains to study are the ones invariant with respect to this action. This motivates the following definition. 
Definition 3.7. An equivariant cochain $\tau \in C^{n}(\mathcal{A}, \Gamma)$ is called cyclic if

$$
\lambda_{\Gamma} \tau=\tau
$$

where $\lambda_{\Gamma}$ is the equivariant cyclic operator.

The vector space of equivariant cyclic $n$-cochains is denoted by $C_{\lambda}^{n}(\mathcal{A}, \Gamma)$

In other words, an equivariant cochain $\tau$ is cyclic iff for any $a_{i} \in \mathcal{A}, g \in \Gamma$ the following relation holds

$$
\tau\left(g^{-1} a_{n}, a_{0}, \ldots, a_{n-1} \mid g\right)=(-1)^{n} \tau\left(a_{0}, a_{1}, \ldots, a_{n} \mid g\right) .
$$

Hence one gets that

$$
C_{\lambda}^{n}(\mathcal{A}, \Gamma)=C^{n}(\mathcal{A}, \Gamma)^{\mathbb{Z} /(n+1) \mathbb{Z}}
$$

As usual, we set

$$
C_{\lambda}^{*}(\mathcal{A}, \Gamma)=\bigoplus_{n \geqslant 0} C_{\lambda}^{n}(\mathcal{A}, \Gamma)
$$

Similarly to the nonequivariant case, the striking phenomenon happening is that the equivariant coboundary operatory $b_{\Gamma}$ is a well defined differential on $C_{\lambda}^{*}(\mathcal{A}, \Gamma)$. Although $b_{\Gamma}$ does not commute with $\lambda_{\Gamma}$, one has

Lemma 3.1.3. The following relations hold

$$
b_{\Gamma}^{\prime}\left(i d-\lambda_{\Gamma}\right)=\left(i d-\lambda_{\Gamma}\right) b_{\Gamma}
$$

and

$$
N_{\Gamma} b_{\Gamma}^{\prime}=b_{\Gamma} N_{\Gamma}
$$

Proof. First observe that the coboundary maps $b_{\Gamma}^{\prime}$ and $b_{\Gamma}$ can be expressed in terms of the cyclic operator and the operator $d_{\Gamma}$ defined in 3.11 more precisely

$$
b_{n}^{\prime}=\sum_{j=0}^{n} \lambda_{n+1}^{-(j+1)} d_{n} \lambda_{n}^{j}
$$

and

$$
b_{n}=\sum_{j=0}^{n+1} \lambda_{n+1}^{-(j+1)} d_{n} \lambda_{n}^{j}
$$

In the above formulas to simplify the notation we omitted the subscript $\Gamma$ throughout and we added the subscript $n$, to stress that the corresponding operator acts on $C^{n}(\mathcal{A}, \Gamma)$. This convention is adopted also in the rest of the proof. The above two formulas are quite easy to derive. 
As a consequence of the above formulas we get the claimed relations. In fact

$$
\begin{array}{r}
b_{n}^{\prime}\left(i d-\lambda_{n}\right)-\left(i d-\lambda_{n+1}\right) b_{n}= \\
\left(\sum_{j=0}^{n} \lambda_{n+1}^{-(j+1)} d_{n} \lambda_{n}^{j}\right)(i d-\lambda)-(i d-\lambda)\left(\sum_{j=0}^{n+1} \lambda_{n+1}^{-(j+1)} d_{n} \lambda_{n}^{j}\right)= \\
=\sum_{j=0}^{n} \lambda_{n+1}^{-(j+1)} d_{n} \lambda_{n}^{j}-\sum_{j=0}^{n} \lambda_{n+1}^{-j} d_{n} \lambda_{n}^{j}+d_{n}-\lambda_{n+1}^{-(n+1)} d_{n}- \\
\sum_{j=0}^{n} \lambda_{n+1}^{-(j+1)} d_{n} \lambda_{n}^{j}-\sum_{j=0}^{n} \lambda_{n+1}^{-(j+1)} d_{n} \lambda_{n}^{j+1}= \\
\sum_{j=0}^{n} \lambda_{n+1}^{-(j+1)} d_{n} \lambda_{n}^{j+1}-\sum_{j=0}^{n} \lambda_{n+1}^{-j} d_{n} \lambda_{n}^{j}+d_{n}-\lambda_{n+1}^{-(n+1)} d_{n}= \\
=\lambda_{n+1}^{-(n+1)} d_{n} \lambda_{n}^{n+1}-d_{n}+d_{n}-\lambda_{n+1}^{-(n+1)} d_{n}=0
\end{array}
$$

Therefore we have shown 3.18 .

The equation 3.19 is proved analogously.

Thus, as immediate consequence of Equation 3.18 of the previous lemma, one gets indeed

$$
b_{\Gamma}\left(C_{\lambda}^{n}(\mathcal{A}, \Gamma)\right) \subseteq C_{\lambda}^{n+1}(\mathcal{A}, \Gamma)
$$

Definition 3.8. The equivariant cyclic complex of $(\mathcal{A}, \Gamma)$ is the subcomplex $\left(C_{\lambda}^{*}(\mathcal{A}, \Gamma), b_{\Gamma}\right)$ of the equivariant Hochschild complex $\left(C^{*}(\mathcal{A}, \Gamma), b_{\Gamma}\right)$.

Its homology is denoted by

$$
H_{\lambda}^{n}(\mathcal{A}, \Gamma)=H_{n}\left(C_{\lambda}^{*}(\mathcal{A}, \Gamma), b_{\Gamma}\right)
$$

\subsection{Equivariant Cyclic Bicomplex}

In this section we will construct the equivariant cyclic bicomplex $C C^{* *}(\mathcal{A}, \Gamma)$ for pairs $(\mathcal{A}, \Gamma)$. As in the nonequivariant case, this bicomplex intertwines the equivariant Hochschild complex and the periodic resolution of period 2 for $\mathbb{Z} /(n+1) \mathbb{Z}$-modules and gives the right framework for defining Hochschild and cyclic cohomology for nonunital $\Gamma$-algebras.

It is not difficult to prove that both definitions of equivariant Hochschild and cyclic cohomology coincide for unital $\Gamma$-algebras, so that one gets two equivalent descriptions of the theories. Moreover we will get easily from the cyclic bicomplex an analogue for (not necessarily unital) $\Gamma$-algebras of the Connes' SBI-sequence, which relates equivariant Hochschild and cyclic cohomology. A comprehensive introduction to the nonequivariant theory can be found in [37] and 18 .

Let $\Gamma$ be a compact Lie group and let $\mathcal{A}$ be a not necessarily unital Fréchet $\Gamma$-algebra. Let us consider $C^{*}(\mathcal{A}, \Gamma)$, the vector space of equivariant cochains on $\mathcal{A}$, and the equivariant cyclic operator $\lambda_{\Gamma}$, the norm operator $N_{\Gamma}$ and finally both Hochschild coboundary operators $b_{\Gamma}^{\prime}, b_{\Gamma}$, as defined in the previous section. 
The above Lemma 3.1 .3 allows us to define the following first quadrant bicomplex, denoted by $C C^{* *}(\mathcal{A}, \Gamma)$

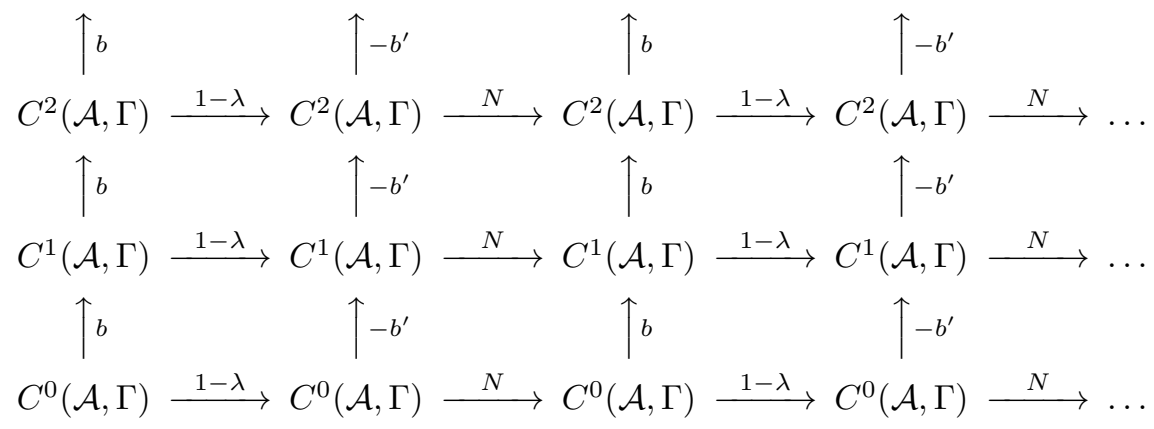

Again, for notational simplicity we have omitted the subscript $\Gamma$ throughout.

Definition 3.9. The bicomplex $C C^{* *}(\mathcal{A}, \Gamma)$ is called the equivariant cyclic bicomplex of the pair $(\mathcal{A}, \Gamma)$.

The total complex of $C C^{* *}(\mathcal{A}, \Gamma)$ will be denoted by $\left(\operatorname{Tot}^{*}(\mathcal{A}, \Gamma), \partial\right)$, where

$$
\operatorname{Tot}^{n}(\mathcal{A}, \Gamma)=\bigoplus_{k=0}^{n} C^{n-k}(\mathcal{A}, \Gamma)
$$

and the differential $\partial=\partial_{v}+\partial_{h}$. An element $x \in \operatorname{Tot}^{n}(\mathcal{A}, \Gamma)$ is, therefore, a $n$-tuple $x=\left(x_{0}, x_{1}, \ldots, x_{n}\right)$, with $x_{k} \in C^{n-k}(\mathcal{A}, \Gamma)$.

Definition 3.10. We define

$$
H C^{n}(\mathcal{A}, \Gamma)=H_{n}\left(\operatorname{Tot}^{*}(\mathcal{A}, \Gamma), \partial\right)
$$

to be the equivariant cyclic cohomology of $(\mathcal{A}, \Gamma)$.

Note that we do not assume the algebra to be unital in the above definition. We set

$$
H C^{*}(\mathcal{A}, \Gamma)=\bigoplus_{n \geq 0} H C^{n}(\mathcal{A}, \Gamma)
$$

Let us now consider only the first two columns of the cyclic bicomplex

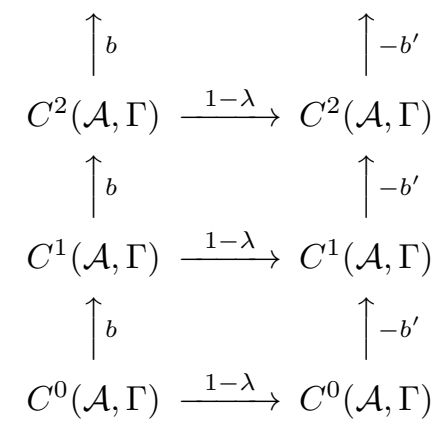

and its total complex $\left(\operatorname{Tot}_{[2]}^{*}, \delta\right)$, where 


$$
\operatorname{Tot}_{[2]}^{n}=C^{n}(\mathcal{A}, \Gamma) \oplus C^{n-1}(\mathcal{A}, \Gamma), \quad n \geq 1, \quad D^{0}=C^{0}(\mathcal{A}, \Gamma)
$$

and

$$
\delta=\left(\begin{array}{cc}
b & 0 \\
i d-\lambda & -b^{\prime}
\end{array}\right)
$$

Definition 3.11. The equivariant Hochschild cohomology $H H^{*}(\mathcal{A}, \Gamma)$ for any (possibly non unital) $\Gamma$-algebra $\mathcal{A}$ is defined as the homology of the cochain complex $\left(\operatorname{Tot}_{[2]}^{*}, \delta\right)$.

If $\mathcal{A}$ has a unit we know that $\left(C^{*}(\mathcal{A}, \Gamma), b_{\Gamma}^{\prime}\right)$ is an acyclic complex, with a chain contraction given explicitly by the degeneracy maps $s$, as defined by Equation 3.10 and therefore the second column of 3.24 can be disregarded for the computation of the $H H^{*}(\mathcal{A}, \Gamma)$.

Thus in this case we reobtain the old definition of equivariant Hochschild cohomology as given in 3.6 , as the homology of $\left(C^{*}(\mathcal{A}, \Gamma), b_{\Gamma}\right)$.

As next step we want to compare the group $H C^{n}(\mathcal{A}, \Gamma)$ with $H_{\lambda}^{n}(\mathcal{A}, \Gamma)$, in the particular case of a unital $\Gamma$-algebra $\mathcal{A}$. These groups are up to isomorphisms the same. This is the content of the following theorem.

Theorem 3.2.1. For any unital $\Gamma$-algebra $\mathcal{A}$ and any $n \in \mathbb{N}$ one has a canonical isomorphism

$$
H C^{n}(\mathcal{A}, \Gamma) \cong H_{\lambda}^{n}(\mathcal{A}, \Gamma)
$$

Proof. Recall that we have shown in Lemma 3.1.1 that the rows of the equivariant cyclic bicomplex $C C^{* *}(\mathcal{A}, \Gamma)$ are acyclic complexes. Thus the only non trivial homology of each row is the homology group $H^{0}\left(C^{*}(\mathcal{A}, \Gamma),(i d-\lambda, N)\right)$. It is an immediate consequence of the definition of equivariant cyclic cochain that

$$
H^{0}\left(C^{k}(\mathcal{A}, \Gamma) ;(i d-\lambda, N)\right)=k e r\left(i d-\lambda_{k}\right)=C_{\lambda}^{k}(\mathcal{A}, \Gamma) .
$$

Moreover, since $C C^{* *}(\mathcal{A}, \Gamma)$ is a bicomplex with horizontal differentials $d^{h}=$ $i d-\lambda_{\Gamma}, N_{\Gamma}$, and vertical differentials $d^{v}=b_{\Gamma},-b_{\Gamma}^{\prime}$, it follows from the relation $d^{h} d^{v}+d^{v} d^{h}=0$ that the equivariant Hochschild coboundary map $b_{\Gamma}$ is a well defined differential on $C^{*}(\mathcal{A}, \Gamma)$, as we already proved in 3.1 .3 .

By a spectral sequence argument (cf. 37], Proposition 1.0.12 and Appendix D), one gets

$$
H C^{n}(\mathcal{A}, \Gamma)=H_{n}\left(\operatorname{Tot}^{*}(\mathcal{A}, \Gamma)\right) \cong H_{n}\left(C_{\lambda}^{*}(\mathcal{A}, \Gamma), b_{\Gamma}\right)=H_{\lambda}^{n}(\mathcal{A}, \Gamma)
$$

Remark 3.5. In the proof of the previous theorem we have obtained the equivariant cyclic complex $\left(C_{\lambda}^{*}(\mathcal{A}, \Gamma), b_{\Gamma}\right)$ of a unital algebra $\mathcal{A}$ as the vertical complex of the homology of the rows of $C C^{* *}(\mathcal{A}, \Gamma)$ (or equivalently as the $E^{1}$-term of the spectral sequence induced by $\left.C C^{* *}(\mathcal{A}, \Gamma)\right)$.

If the algebra $\mathcal{A}$ is nonunital, we can define its equivariant cyclic complex as 
being the $E^{1}$-term of the spectral sequence induced by the cyclic bicomplex of $(\mathcal{A}, \Gamma)$. Explicitly, this complex is $\left(C_{\lambda}^{*}(\mathcal{A}, \Gamma), b_{\Gamma}\right)$, where $C_{\lambda}^{n}(\mathcal{A}, \Gamma)$ is the vector space of cyclic equivariant $n$-cochains of $(\mathcal{A}, \Gamma)$ and $b_{\Gamma}$ is the usual equivariant Hochschild differential.

Hence, we get that the equivariant cyclic cohomology of a nonunital algebra $\mathcal{A}$ can be computed also through its equivariant cyclic complex $\left(C_{\lambda}^{*}(\mathcal{A}, \Gamma), b_{\Gamma}\right)$.

This is implicitly used in [7], where equivariant Hochschild and cyclic cohomology are defined explicitly only in the unital case.

Let now $\mathcal{B}$ be another Fréchet $\Gamma$-algebra. For any continuous $\Gamma$-equivariant algebra homomorphism $\psi: \mathcal{A} \rightarrow \mathcal{B}$, we define

$$
\left(\psi^{*} \tau\right)\left(a_{0}, a_{1}, \ldots, a_{n} \mid h\right)=\tau\left(\psi\left(a_{0}\right), \psi\left(a_{1}\right), \ldots, \psi\left(a_{n}\right) \mid h\right)
$$

for every $\tau \in C^{n}(\mathcal{B}, \Gamma), a_{i} \in \mathcal{A}, h \in \Gamma$.

Using the fact that $\psi$ is an equivariant homomorphism, it is immediate to check that $\psi^{*}$ gives rise to a cochain map of degree 0

$$
\psi^{*}: C_{\lambda}^{n}(\mathcal{B}, \Gamma) \rightarrow C_{\lambda}^{n}(\mathcal{A}, \Gamma)
$$

which in turn induces a well- defined map

$$
\psi^{*}: H C^{n}(\mathcal{B}, \Gamma) \rightarrow H C^{n}(\mathcal{A}, \Gamma)
$$

Thus we have shown

Lemma 3.2.2. Let $\Gamma$ be a compact Lie group. For every $n \in \mathbb{N}, H C^{n}(\cdot, \Gamma)$ is a functor on the category of Fréchet $\Gamma$-algebras and equivariant continuous algebra homomorphisms to the category of abelian groups and group homomorphisms.

If we assume the group to be finite, all the results of the preceding sections hold for any $\Gamma$-algebra without topology.

\subsection{The equivariant SBI-sequence and the peri- odic theory}

Besides the possibility to define equivariant Hochschild and cyclic cohomology for nonunital algebras, another advantage of working with the equivariant cyclic bicomplex is that it enables to show very simply a long exact sequence that relates the equivariant Hochchild cohomology groups with the equivariant cyclic ones. This sequence is the so-called equivariant $S B I$-sequence and it is important because it reduces the problem of computing the equivariant cyclic cohomology groups to the computation of the equivariant Hochschild cohomology. More precisely, once the equivariant Hochschild cohomology has been computed, then applying the $S B I$-sequence one can compute in principle the equivariant cyclic cohomology inductively.

In this section, we will provide the details. Furthermore, we will introduce 
equivariant periodic cyclic cohomology by means of the periodicity operator $S_{\Gamma}$, also appearing in the $S B I$-sequence.

Let $\mathcal{A}$ be a Fréchet, possibly nonunital $\Gamma$-algebra. Let us consider the cyclic bicomplex $C C^{* *}(\mathcal{A}, \Gamma)$ and its total complex $\left(\operatorname{Tot}^{*}(\mathcal{A}, \Gamma), \partial\right)$. Recall that an element $x \in \operatorname{Tot}^{n}(\mathcal{A}, \Gamma)$ is a $n$-tuple $x=\left(x_{0}, x_{1}, \ldots, x_{n}\right)$, where $x_{k} \in C^{n-k}(\mathcal{A}, \Gamma)$. One can define the cochain map $S_{\Gamma}: \operatorname{Tot}^{*}(\mathcal{A}, \Gamma) \rightarrow \operatorname{Tot}^{*}(\mathcal{A}, \Gamma)$ defined by

$$
S_{\Gamma}\left(x_{0}, x_{1}, \ldots, x_{n}\right)=\left(0,0, x_{0}, x_{1}, \ldots, x_{n}\right)
$$

for any $\left(x_{0}, x_{1}, \ldots, x_{n}\right) \in \operatorname{Tot}^{n}(\mathcal{A}, \Gamma)$. It is immediate to see that $S_{\Gamma}$ is a cochain map of degree 2 .

Moreover, taking the total complex $\left(\operatorname{Tot}_{[2]}^{*}(\mathcal{A}, \Gamma), \delta\right)$ of the bicomplex 3.24, we define $I: \operatorname{Tot}^{*}(\mathcal{A}, \Gamma) \rightarrow \operatorname{Tot}_{[2]}^{*}(\mathcal{A}, \Gamma)$ to be the map given by projecting, i.e.

$$
I\left(x_{0}, x_{1}, \ldots, x_{n}\right)=\left(x_{0}, x_{1}\right),
$$

for all $x \in \operatorname{Tot}^{n}(\mathcal{A}, \Gamma)$. In particular, if $x_{0} \in \operatorname{Tot}^{0}(\mathcal{A}, \Gamma)$, then $I\left(x_{0}\right)=x_{0}$.

Thus, we get a short exact sequence of cochain complexes

$$
0 \rightarrow \operatorname{Tot}^{*}(\mathcal{A}, \Gamma) \stackrel{S_{\Gamma}}{\longrightarrow} \operatorname{Tot}^{*}(\mathcal{A}, \Gamma) \stackrel{I}{\rightarrow} \operatorname{Tot}_{[2]}^{*}(\mathcal{A}, \Gamma) \rightarrow 0,
$$

where, however, the map $S_{\Gamma}$ is of degree 2 .

To get cochain maps of degree 0 in the above sequence, we perform the following trick. Let us define the cochain complex $\left(\operatorname{Tot}^{*}(\mathcal{A}, \Gamma)[-2], \partial^{\prime}\right)$ by

$$
\begin{array}{rr}
\operatorname{Tot}^{n}(\mathcal{A}, \Gamma)[-2]=\operatorname{Tot}^{n-2}(\mathcal{A}, \Gamma), & n \geq 2 \\
\operatorname{Tot}^{n}(\mathcal{A}, \Gamma)[-2]=0, & n=0,1
\end{array}
$$

with differential $\partial_{n}^{\prime}=\partial_{n}$, for $n \geq 2$, and $\partial^{\prime}=0$, if $n=0,1$. Morally, we have simply shifted $\operatorname{Tot}^{*}(\mathcal{A}, \Gamma)$ to the left.

Now, it is clear that $S_{\Gamma}: \operatorname{Tot}^{*}(\mathcal{A}, \Gamma)[-2] \rightarrow \operatorname{Tot}^{*}(\mathcal{A}, \Gamma)$, extended in the obvious way, is a degree 0 map of cochain complexes and one gets the short exact sequence

$$
0 \rightarrow \operatorname{Tot}^{*}(\mathcal{A}, \Gamma)[-2] \stackrel{S_{\Gamma}}{\longrightarrow} \operatorname{Tot}^{*}(\mathcal{A}, \Gamma) \stackrel{I}{\rightarrow} \operatorname{Tot}_{[2]}^{*}(\mathcal{A}, \Gamma) \rightarrow 0
$$

Proposition 3.3.1. (equivariant SBI-sequence) The short exact sequence 3.34 gives rise to the long exact sequence in cohomology

$$
\begin{aligned}
\cdots \rightarrow & H C^{n-2}(\mathcal{A}, \Gamma) \stackrel{S_{\Gamma}}{\longrightarrow} H C^{n}(\mathcal{A}, \Gamma) \stackrel{I}{\rightarrow} H H^{n}(\mathcal{A}, \Gamma) \stackrel{B_{\Gamma}}{\longrightarrow} H C^{n-1}(\mathcal{A}, \Gamma) \\
& \stackrel{S_{\Gamma}}{\longrightarrow} H C^{n+1}(\mathcal{A}, \Gamma) \stackrel{I}{\rightarrow} H H^{n+1}(\mathcal{A}, \Gamma) \stackrel{B_{\Gamma}}{\longrightarrow} H C^{n}(\mathcal{A}, \Gamma) \rightarrow \ldots
\end{aligned}
$$

In the proposition above, the maps in cohomology are denoted with the same symbol of the maps on cochains.

Proof. This is just the long exact sequence in cohomology associated to the short exact sequence 3.34 . 
As already mentioned, the $S B I$-sequence allows in many cases to compute explicitly the equivariant cyclic cohomology, if the Hochschild cohomolgy is known.

The operators $S_{\Gamma}: H C^{n}(\mathcal{A}, \Gamma) \rightarrow H C^{n+2}(\mathcal{A}, \Gamma)$ and $B_{\Gamma}: H H^{n}(\mathcal{A}, \Gamma) \rightarrow$ $H C^{n-1}(\mathcal{A}, \Gamma)$ are very important in equivariant cyclic cohomology. The latter leads to another approach to the cyclic theory and we refer to [46] for remarks and details about this point.

The operator $S_{\Gamma}$, also called periodicity operator, allows to define equivariant periodic cyclic cohomology. More precisely, note that by definition of $S_{\Gamma}$ one can give the sets $\left\{\left(H C^{n+2 l}(\mathcal{A}, \Gamma), S_{\Gamma}\right)\right\}_{l \in \mathbb{N}}$, where $n=0,1$, a natural structure of direct system. Hence, we can set the following

Definition 3.12. Given a $\Gamma$-algebra $\mathcal{A}$, its equivariant periodic cyclic cohomology $H P^{n}(\mathcal{A}, \Gamma)$, for $n=0,1$ is defined to be

$$
H P^{n}(\mathcal{A}, \Gamma)=\underset{S_{\Gamma}}{\lim _{\longrightarrow}} H C^{n+2 l}(\mathcal{A}, \Gamma)
$$

\subsubsection{Some Remarks and construction}

So far we have assumed the algebras to be Fréchet $\Gamma$-algebras.

This choice was mainly done for the sake of simplicity, in order to emphasize the algebraic aspects of the theory.

It would be desiderable to extend the equivariant cyclic theory to more general locally convex algebras, since in many geometrically motivated problems such algebras arise naturally. For instance, in Foliation theory one is interested in the convolution algebras of compactly supported smooth functions on the variuos Lie groupoids associated to a foliation. These are locally convex algebras with separately continuous multiplication.

For these latter algebras, however, one cannot simply follow the same pattern used for Fréchet algebras, since many technical problems arise. For instance, the equivariant Hochschild differential of a continuous equivariant cochain is no longer continuous, but in general only separately continuous. Hence, we do not get a well-defined cochain complex. Moreover, for such algebras one does not have a natural choice of a well-behaved topological tensor product. The lack of the tensor product makes difficult (or even impossible) to prove the properties we would like equivariant cyclic theories to have.

We point out that such technical problems can be solved by considering bornologies on the algebras, rather than topologies. A bornology on a vector space is a suitable collection of subsets, playing the same role as that of bounded subsets in a locally convex space. Every locally convex algebra carries natural bornologies, determined by the locally convex topology. The point is that bornological algebras have better functorial features, which make possible to overcome the difficulties one encounters proceeding with locally convex topologies.

We refer the interested reader to [46, where equivariant cyclic theories are defined for complete bornological $\Gamma$-algebras and many interesting properties are established. 
In spite of the fact that we cannot easily define equivariant cyclic cohomology groups for locally convex $\Gamma$-algebras, we can still consider equivariant cyclic cocycles on the algebra. More precisely, given a locally convex $\Gamma$-algebra $\mathcal{A}$, a continuous $(n+1)$-linear map $\tau: \mathcal{A} \times \cdots \times \mathcal{A} \rightarrow C(\Gamma)$ invariant with respect to the action of $\Gamma$ on $\mathcal{A}$ and $C(\Gamma)$ is said to be an equivariant Hochschild $n$-cochain of $\mathcal{A}$, or simply an equivariant cochain.

The operators $\lambda_{\Gamma}$ and $b_{\Gamma}$, defined as above, can be applied to an equivariant $n$-cochain. Thus, it makes sense to define an equivariant cyclic cocycle as follows.

Definition 3.13. Given a locally convex $\Gamma$-algebra $\mathcal{A}$, An equivariant cyclic $n$-cocycle is an equivariant $n$-cochain $\tau$ on $\mathcal{A}$ such that $\lambda_{\Gamma} \tau=\tau$ and $b_{\Gamma} \tau=0$.

In the next section we shall see that any equivariant cyclic cocycle on $(\mathcal{A}, \Gamma)$ determines an additive map on the equivariant $K$-theory of $\mathcal{A}$. This is crucial for being able to define higher Lefschetz invariants for foliated $\Gamma$-manifolds.

Now, to end this section we shall prove three lemmas that provide simple constructions to obtain equivariant cyclic cocycles on a special class of locally convex algebras, namely LF-algebras. A LF-space is a locally convex vector space $V$ for which there is an increasing sequence of subspaces $\left(V_{n}\right)_{n \in \mathbb{N}}$ such that each $V_{n}$ is a Fréchet space with respect to the subspace topology, the union $\bigcup_{n \in \mathbb{N}} V_{n}=V$ and $V$ carries the finest locally convex topology making the inclusions $V_{n} \rightarrow V$ continuous for every $n \in \mathbb{N}$. A LF-algebra is a LF-space endowed with a separately continuous multiplication. We refer to [46, pag. 15, and the references therein for further remarks and properties of such algebras.

Important examples of LF-algebras are the convolution algebras of compactly supported smooth functions on Lie groupoids.

The first lemma we prove is a straightforward generalization of Proposition 4.15 in [7. Let $\mathcal{A}$ be a LF-algebra and suppose that there is a $C^{*}$-algebra $B$ which contains $\mathcal{A}$ as a dense subalgebra.

Denote the multiplier algebra of $B$ by $M(B)$ and suppose there is a continuous group homomorphism

$$
\Psi: \Gamma \rightarrow G L(M(B))
$$

where $G L(M(B))$ denotes the multiplicative group of $M(B)$ and $\Gamma$ is a compact Lie group.

Assume now that for all $h \in \Gamma$ the multiplier $\Psi(g)$ satisfies

$$
\Psi(g) \mathcal{A} \subseteq \mathcal{A} \quad \mathcal{A} \Psi(g) \subseteq \mathcal{A}
$$

and furthermore that the linear actions defined by left and right multiplication with $\Psi(g)$ take value in the continuous linear operators of $\mathcal{A}$ and happen to be strongly continuous (with respect to the locally convex topology of $\mathcal{A}$ ).

Then we get a $\Gamma$-action on $\mathcal{A}$ given by the formula

$$
g \cdot a=\Psi(g) a \Psi\left(g^{-1}\right)
$$

for all $a \in \mathcal{A}, g \in \Gamma$. 
Example 3.1. The pivotal example of the above situation is the following. Suppose to have a Lie groupoid $G$ on which $\Gamma$ acts by inner automorphisms defined through the group homomorphism $\sigma: \Gamma \rightarrow B i s(G)$. Now, taking $\mathcal{A}=$ $C_{c}^{\infty}\left(G, \Omega^{1 / 2} G\right)$ with its natural LF-space structure and $B=C_{r}^{*}(G)$ the groupoid $C^{*}$-algebra, we have constructed in Proposition 2.1.14 the multiplier $S(g) \in$ $M\left(C_{r}^{*}(G)\right)$, for all $g \in \Gamma$. Each multiplier preserves $C_{c}^{\infty}\left(G, \Omega^{1 / 2} G\right)$ and it gives rise to linear actions with values in the continuous operators of $C_{c}^{\infty}\left(G, \Omega^{1 / 2} G\right)$ and which are strongly continuous.

Lemma 3.3.2. Let $k>0$ and let $\tau$ be a continuous cyclic $k$-cocycle on $\mathcal{A}$ satisfying

$$
\tau\left(a^{0}, a^{1}, \ldots, a^{i} \cdot \Psi(g), a^{i+1}, \ldots, a^{k}\right)=\tau\left(a^{0}, a^{1}, \ldots, a^{i}, \Psi(g) \cdot a^{i+1}, \ldots, a^{k}\right)
$$

for every $a^{i} \in \mathcal{A}, \quad g \in \Gamma$. The expression

$$
\varphi\left(a^{0}, \ldots, a^{k} \mid g\right)=\tau\left(\Psi(g) \cdot a^{0}, a^{1}, \ldots, a^{k}\right)
$$

defines an equivariant cyclic cocycle on $(\mathcal{A}, \Gamma)$.

Furthermore, if $\tau$ is a cyclic 0 -cocycle on $\mathcal{A}$ such that $\tau(\Psi(g) \cdot a)=\tau(a \cdot \Psi(g))$, then

$$
\varphi(a \mid g)=\tau(\Psi(g) \cdot a)
$$

defines an equivariant cyclic 0 -cocycle on $(\mathcal{A}, \Gamma)$.

Proof. Let us consider first the case where $k>0$.

- We have to show that $\varphi$ is $\Gamma$-invariant, i.e. $\forall a^{i} \in \mathcal{A}, g, h \in \Gamma$

$$
\varphi\left(h \cdot a^{0}, h \cdot a^{1}, \ldots, h \cdot a^{k} \mid h g h^{-1}\right)=\varphi\left(a^{0}, a^{1}, \ldots, a^{k} \mid g\right) .
$$

By definition of the action and by the relation 3.38 , one gets

$$
\begin{gathered}
\varphi\left(h \cdot a^{0}, h \cdot a^{1}, \ldots, h \cdot a^{k} \mid h g h^{-1}\right)=\tau\left(\Psi\left(h g h^{-1}\right)\left(h \cdot a^{0}\right), h \cdot a^{1}, \ldots, h \cdot a^{k}\right)= \\
=\tau\left(\Psi(h) \Psi(g) \Psi\left(h^{-1}\right) \Psi(h) a^{0} \Psi\left(h^{-1}\right), \Psi(h) a^{1} \Psi\left(h^{-1}\right), \ldots, \Psi(h) a^{k} \Psi\left(h^{-1}\right)\right)= \\
=\tau\left(\Psi(h) \Psi(g) a^{0}, a^{1}, \ldots, a^{k} \Psi\left(h^{-1}\right)\right) .
\end{gathered}
$$

Finally, by using the fact that $\tau$ is cyclic we deduce that

$$
\begin{gathered}
\tau\left(\Psi(h) \Psi(g) a^{0}, a^{1}, \ldots, a^{k} \Psi\left(h^{-1}\right)\right)=\tau\left(\Psi(g) a^{0}, a^{1}, \ldots, a^{k}\right)= \\
=\varphi\left(a^{0}, a^{1}, \ldots, a^{k} \mid g\right) .
\end{gathered}
$$

- Let us now show that $\varphi$ is equivariantly cyclic.

$$
\begin{gathered}
\left(\lambda_{\Gamma} \varphi\right)\left(a^{0}, \ldots, a^{k} \mid g\right)=(-1)^{k} \varphi\left(g^{-1} \cdot a^{k}, a^{0}, \ldots, a^{k+1} \mid g\right)= \\
=(-1)^{k} \tau\left(\Psi(g)\left(g^{-1} a^{k}\right), a^{1}, \ldots, a^{k-1}\right)=(-1)^{k} \tau\left(a^{k} \Psi(g), a^{0}, \ldots, a^{k-1}\right)= \\
=(-1)^{k} \tau\left(a^{k}, \Psi(g) a^{0}, \ldots, a^{k-1}\right) .
\end{gathered}
$$

By the cyclicity of $\tau$ it follows that

$$
\begin{gathered}
(-1)^{k} \tau\left(a^{k}, \Psi(g) a^{0}, \ldots, a^{k-1}\right)=\tau\left(\Psi(g) a^{0}, a^{1}, \ldots, a^{k+1}, a^{k}\right)= \\
=\varphi\left(a^{0}, a^{1}, \ldots, a^{k} \mid g\right) .
\end{gathered}
$$


- The proof of the fact that $\varphi$ is an equivariant cocycle follows again from the definitions, similarly as we did in the previous two steps.

The case $k=0$ follows from similar computations.

The continuity of the equivariant cocycle $\varphi$ follows immediately from the continuity of the action and of $\tau$.

Lemma 3.3.3. Let $\mathcal{A}$ be a $\Gamma$-algebra as considered above. Let us assume that there is a one-parameter group $\left(\sigma_{t}\right)_{t \in \mathbb{R}} \subseteq$ Aut $(B)$ of automorphisms of the $C^{*}$-algebra $B$ such that

- $\sigma_{t}(\mathcal{A}) \subseteq \mathcal{A}$, for all $t \in \mathbb{R}$

- the infinitesimal generator $D$ of $\left(\sigma_{t}\right)_{t \in \mathbb{R}}$ determines a derivation of $\mathcal{A}$ that is continuous with respect to the locally convex topology.

Moreover, suppose that the two actions satisfy the following compatibility relations

$$
\begin{aligned}
& \sigma_{t}(\Psi(g) a)=\Psi(g) \sigma_{t}(a) \\
& \sigma_{t}(a \Psi(g))=\sigma_{t}(a) \Psi(g)
\end{aligned}
$$

for all $t \in \mathbb{R}, g \in \Gamma, a \in \mathcal{A}$.

If $\tau$ is a continuous cyclic 1 -cocycle on $\mathcal{A}$ satisfying Relation 3.38, then the formula

$$
\frac{d}{d t}_{\mid t=0} \sigma_{t}^{*}(\tau)\left(\Psi(g) a^{0}, a^{1}\right)
$$

defines an equivariant cyclic 1 - cocycle on $(\mathcal{A}, \Gamma)$.

Proof. First, we observe that

$$
\begin{gathered}
\dot{\tau}\left(a^{0}, a^{1}\right)=\frac{d}{d t}{ }_{\mid t=0} \sigma_{t}^{*}(\tau)\left(a^{0}, a^{1}\right)=\lim _{t \rightarrow 0} \frac{\tau\left(\sigma_{t}\left(a^{0}\right), \sigma_{t}\left(a^{1}\right)\right)-\tau\left(a^{0}, a^{1}\right)}{t}= \\
=\tau\left(D\left(a^{0}\right), a^{1}\right)+\tau\left(a^{0}, D\left(a^{1}\right)\right)
\end{gathered}
$$

for $a^{i} \in \mathcal{A}$. It can be easily checked that $\dot{\tau}$ is a cyclic cocycle.

Now applying the hypothesis and Relation 3.38 , one has that

$$
\begin{aligned}
& \dot{\tau}\left(a^{0} \Psi(g), a^{1}\right)=\tau\left(D\left(a^{0} \Psi(g)\right), a^{1}\right)+\tau\left(a^{0} \Psi(g), D\left(a^{1}\right)\right)= \\
& =\tau\left(D\left(a^{0}\right), \Psi(g) a^{1}\right)+\tau\left(a^{0}, D\left(\Psi(g) a^{1}\right)\right)=\dot{\tau}\left(a^{0}, \Psi(g) a^{1}\right) .
\end{aligned}
$$

Now setting for $g \in \Gamma$

$$
\dot{\varphi}\left(a^{0}, a^{1} \mid g\right)=\dot{\tau}\left(\Psi(g) a^{0}, a^{1}\right) .
$$

the claim follows from Lemma 3.3.2. 
Now, if a cyclic 1-cocycle $\tau$ is invariant under a one-parameter group of automorphisms $\left(\sigma_{t}\right)_{t \in \mathbb{R}}$, one can canonically construct a 2 -cyclic cocycle out of $\rho$ and $\left(\sigma_{t}\right)$. More precisely, let $\rho$ be a cyclic 1 -cocycle on $\mathcal{A}$ and assume that the infinitesimal generator $D$ of $\left(\sigma_{t}\right)$ is such that $\mathcal{A} \subseteq \operatorname{dom}(D)$. One says that $\rho$ is invariant under $\left(\sigma_{t}\right)$ if $\dot{\rho}=0$. In this case, the formula

$$
i_{D}(\rho)\left(a^{0}, a^{1}, a^{2}\right)=\rho\left(D\left(a^{2}\right) a^{0}, a^{1}\right)-\rho\left(a^{0} D\left(a^{1}\right), a^{2}\right)
$$

defines a cyclic 2 -cocycle on $\mathcal{A}$.

The cocycle $i_{D_{\alpha}}(\rho)$ is called the contraction of $\rho$ and we refer to [16], Lemma 7.6 for details.

We have the following

Lemma 3.3.4. With the same notation and hypothesis of the previous two lemmas, if the cyclic cocycle $\dot{\tau}$ is invariant under the one-parameter group $\left(\sigma_{t}\right)_{t \in \mathbb{R}}$, i.e. $\ddot{\tau}=0$, then its contraction $i_{D}(\dot{\tau})$ is the evaluation at the identity of an equivariant 2-cyclic cocycle given by

$$
\psi\left(a^{0}, a^{1}, a^{2} \mid g\right)=i_{D}(\dot{\tau})\left(\Psi(g) a^{0}, a^{1}, a^{2}\right)
$$

for all $a^{i} \in \mathcal{A}, g \in \Gamma$.

Proof. Recall (17]) that

$$
i_{D}(\dot{\tau})\left(a^{0}, a^{1}, a^{2}\right)=\dot{\tau}\left(D\left(a^{2}\right) a^{0}, a^{1}\right)-\dot{\tau}\left(a^{0} D\left(a^{1}\right), a^{2}\right) .
$$

From the proof of Lemma 3.3 .3 we know that $\dot{\tau}$ satisfies the Relation 3.38. Using this and the fact that $i_{D}(\dot{\tau})$ is a cocycle, it is straightforward to check that $\psi$ is an equivariant Hochschild cocycle.

Let us prove the cyclicity. One has

$$
\begin{gathered}
\left(\lambda_{\Gamma} \psi\right)\left(a^{0}, a^{1}, a^{2} \mid g\right)=\psi\left(g^{-1} a^{2}, a^{0}, a^{1} \mid g\right)= \\
=i_{D}(\dot{\tau})\left(\Psi(g)\left(g^{-1} a^{2}\right), a^{0}, a^{1}\right)=i_{D}(\dot{\tau})\left(a^{2} \Psi(g), a^{0}, a^{1}\right)= \\
=\dot{\tau}\left(D\left(a^{1}\right)\left(a^{2} \Psi(g)\right), a^{0}\right)-\dot{\tau}\left(\left(a^{2} \Psi(g)\right) D\left(a^{0}\right), a^{1}\right) .
\end{gathered}
$$

For notational simplicity we set $b=a^{2} \Psi(g)$. Then using that $D$ is a derivation and that $\dot{\tau}$ is a Hochschild cocycle, i.e. $\dot{\tau}\left(b a^{0}, D\left(a^{1}\right)\right)-\dot{\tau}\left(b, a^{0} D\left(a^{1}\right)\right)+$ $\dot{\tau}\left(D\left(a^{1}\right) b, a^{0}\right)=0$, we get

$$
\begin{gathered}
\dot{\tau}\left(D\left(a^{1}\right) b, a^{0}\right)-\dot{\tau}\left(b D\left(a^{0}\right), a^{1}\right)= \\
=\dot{\tau}\left(b, a^{0} D\left(a^{1}\right)\right)-\dot{\tau}\left(b a^{0}, D\left(a^{1}\right)\right)+\dot{\tau}\left(D(b) a^{0}, a^{1}\right)-\dot{\tau}\left(D\left(b a^{0}\right), a^{1}\right)= \\
=\dot{\tau}\left(D(b) a^{0}, a^{1}\right)-\dot{\tau}\left(a^{0} D\left(a^{1}\right), b\right),
\end{gathered}
$$

since $\dot{\tau}\left(b a^{0}, D\left(a^{1}\right)\right)+\dot{\tau}\left(D\left(b a^{0}\right), a^{1}\right)=0$ by the invariance of $\dot{\tau}$ under the 1 -parameter group. Applying Relation 3.38, the result follows. 


\subsection{The pairing with equivariant K-theory}

In this section we will show that any equivariant cyclic cocycle on the pair $(\mathcal{A}, \Gamma)$ gives rise to an additive map defined on the equivariant $K$-theory $K_{0}^{\Gamma}(\mathcal{A})$ with values in the conjugation invariant continuous functions on $\Gamma$.

This pairing is one of the most basic tools for dealing with equivariant indextheoretical problems within the framework of Noncommutative Geometry.

In the setting of foliated manifolds, the equivariant indices of elliptic $\Gamma$-invariant longitudinal differential operators have been defined to be elements of the equivariant K-group of the foliation $C^{*}$-algebra and of the smooth convolution algebra (see 2.2.3). The additive maps constructed out equivariant cyclic cocycles will allow to extract both analytical and geometric informations out of these index classes.

This section is largely based on [7] and on [17, Chapter III, Section 3.

Let $(V, \rho)$ be a finite dimensional unitary representation of the compact Lie group $\Gamma$. By equation 2.22 we already know that the algebra $\operatorname{End}(V)$ has a natural $\Gamma$-action given by conjugation, which we will simply denote by $h T h^{-1}$, for any $T \in \operatorname{End}(V), h \in \Gamma$.

Moreover, it has the natural equivariant 0-cyclic cocycle $\operatorname{Tr}(A \rho(h))$, for any $h \in \Gamma, A \in \operatorname{End}(V)$, provided by "twisting" the trace with the representation. In what follows, we will also denote the equivariant trace by $\operatorname{Tr}(A h)$, thinking of $h$ as $\rho(h)$.

If $\mathcal{A}$ is a locally convex $\Gamma$-algebra and $\tau$ is an equivariant cochain, we define the map $\tau \sharp T r$ on $\mathcal{A} \otimes \operatorname{End}(V)$ by setting

$$
\tau \sharp \operatorname{Tr}\left(a^{0} \otimes A^{0}, a^{1} \otimes A^{1}, \ldots, a^{n} \otimes A^{n} \mid h\right)=\tau\left(a^{0}, \ldots, a^{n} \mid h\right) \operatorname{Tr}\left(A^{0} \cdots A^{n} \rho(h)\right)
$$

for any $a^{i} \in \mathcal{A}, A^{i} \in \operatorname{End}(V), h \in \Gamma$, and then extending it by linearity.

Lemma 3.4.1. The cochain $\tau \sharp T r$ is continuous and equivariant, i.e. it is an equivariant Hochschild cochain. Moreover one has

$$
b_{\Gamma}(\tau \sharp T r)=\left(b_{\Gamma} \tau\right) \sharp T r
$$

Therefore, if $\tau$ is an equivariant Hochschild cocycle, so is $\tau \sharp T r$.

Proof. The new cochain $\tau \sharp T r$ is clearly continuous. Besides, we get

$$
\begin{gathered}
(\tau \sharp T r)\left(h\left(a^{0} \otimes A^{0}\right), h\left(a^{1} \otimes A^{1}\right), \ldots, h\left(a^{n} \otimes A^{n}\right) \mid h g h^{-1}\right)= \\
=(\tau \sharp T r)\left(h a^{0} \otimes h A^{0} h^{-1}, \ldots, h a^{n} \otimes h A^{n} h^{-1} \mid h g h^{-1}\right)= \\
=\tau\left(h a^{0}, \ldots, h a^{n} \mid h g h^{-1}\right) \operatorname{Tr}\left(h\left(A^{0} A^{1} \cdots A^{n}\right) g h^{-1}\right)= \\
=\tau\left(a^{0}, a^{1}, \ldots, a^{n} \mid g\right) \operatorname{Tr}\left(\left(A^{0} A^{1} \cdots A^{n}\right) g\right)= \\
=(\tau \sharp T r)\left(a^{0} \otimes A^{0}, a^{1} \otimes A^{1}, \ldots, a^{n} \otimes A^{n} \mid g\right),
\end{gathered}
$$

which proves that the cochain $\tau \sharp T r$ is equivariant.

Let us now prove the equation 3.47 . One has

$$
\left[b_{\Gamma}(\tau \sharp T r)\right]\left(a^{0} \otimes A^{0}, a^{1} \otimes A^{1}, \ldots, a^{n+1} \otimes A^{n+1} \mid g\right)=
$$




$$
\begin{gathered}
=\sum_{i=0}^{n}(-1)^{i}(\tau \sharp T r)\left(a^{0} \otimes A^{0}, \ldots, a^{i} a^{i+1} \otimes A^{i} A^{i+1}, \ldots, a^{n+1} \otimes A^{n+1} \mid g\right)+ \\
+(-1)^{n+1}(\tau \sharp T r)\left(\left(g^{-1}\left(a^{n+1} \otimes A^{n+1}\right)\right)\left(a^{0} \otimes A^{0}\right), a^{1} \otimes A^{1}, \ldots, a^{n} \otimes A^{n} \mid g\right)= \\
=\sum_{i=0}^{n}(-1)^{i} \tau\left(a^{0}, \ldots, a^{i} a^{i+1}, \ldots, a^{n+1} \mid g\right) \operatorname{Tr}\left(\left(A^{0} A^{1} \cdots A^{n+1}\right) g\right)+ \\
+(-1)^{n+1} \tau\left(\left(g^{-1} a^{n+1}\right) a^{0}, a^{1}, \ldots, a^{n} \mid g\right) \operatorname{Tr}\left(\left(A^{0} A^{1} \cdots A^{n+1}\right) g\right)= \\
\left(\left(b_{\Gamma} \tau\right) \sharp T r\right)\left(a^{0} \otimes A^{0}, a^{1} \otimes A^{1}, \ldots, a^{n+1} \otimes A^{n+1} \mid g\right) .
\end{gathered}
$$

This completes the proof.

Lemma 3.4.2. If $\tau \in C_{\lambda}^{n}(\mathcal{A}, \Gamma)$, then $\tau \sharp T r$ is an equivariant cyclic cochain on $\mathcal{A} \otimes \operatorname{End}(V)$.

Proof. Since $\tau$ is cyclic, then one gets

$$
\begin{gathered}
(\tau \sharp T r)\left(g^{-1}\left(a^{n} \otimes A^{n}\right), a^{0} \otimes A^{0}, a^{1} \otimes A^{1}, \ldots, a^{n-1} \otimes A^{n-1} \mid g\right)= \\
=\tau\left(g^{-1} a^{n}, a^{0}, a^{1}, \ldots, a^{n-1} \mid g\right) \operatorname{Tr}\left(A^{n} g A^{0} A^{1} A^{n-1}\right)= \\
=(-1)^{n} \tau\left(a^{0}, a^{1}, \ldots, a^{n} \mid g\right) \operatorname{Tr}\left(\left(A^{0} \cdots A^{n}\right) g\right)= \\
=(-1)^{n}(\tau \sharp T r)\left(a^{0} \otimes A^{0}, a^{1} \otimes A^{1}, \ldots, a^{n} \otimes A^{n} \mid g\right) .
\end{gathered}
$$

Using together the lemmas above, one immediately deduces that if $\tau$ is an equivariant cyclic cocycle, so is $\tau \sharp T r$ as well.

Since the group $\Gamma$ is compact, the equivariant $K$-theory can be defined by means of $\Gamma$-invariant idempotents $e \in \mathcal{A} \otimes \operatorname{End}(V)$, for all finite dimensional unitary representations $V$ of $\Gamma$ (see 2.2.1.

We are now ready to see how an equivariant cyclic cocycle determines an addtive map on equivariant $K$-theory.

Let $\mathcal{A}$ be a locally convex $\Gamma$-algebra and let $\tau$ be an equivariant cyclic $2 k$-cocycle on $(\mathcal{A}, \Gamma)$. For any finite dimensional unitary representation $(V, \rho)$ of $\Gamma$ and for any $\Gamma$-invariant idempotent $e \in \mathcal{A} \otimes \operatorname{End}(V)$ and any $h \in \Gamma$, one sets

$$
\langle e, \tau\rangle(h)=(\tau \sharp T r)(e, \ldots, e \mid h) .
$$

Proposition 3.4.3. ([7])

1. With the notation above, the mapping $\langle e, \tau\rangle$ defines a continuous conjugation invariant function on $\Gamma$, i.e $\langle e, \tau\rangle \in C(\Gamma)^{\Gamma}$.

2. The formula 3.48 descends a well defined additive map

$$
\langle\cdot, \tau\rangle: K_{0}^{\Gamma}(\mathcal{A}) \rightarrow C(\Gamma)^{\Gamma}
$$


3. If the algebra $\mathcal{A}$ is a Fréchet algebra, the map 3.49 does only depend on the cohomology class of $\tau$ and it induces a pairing

$$
K_{0}^{\Gamma}(\mathcal{A}) \times H C^{e v}(\mathcal{A}, \Gamma) \rightarrow C(\Gamma)^{\Gamma}
$$

between the equivariant $K$-theory and equivariant cyclic cohomology.

As observed in [7, when $\mathcal{A}$ is not unital, the statement in the second item means that if $e \in \mathcal{A}^{+} \otimes \operatorname{End}(V)$ and $e^{\prime} \in \mathcal{A}^{+} \otimes \operatorname{End}\left(V^{\prime}\right)\left(\mathcal{A}^{+}\right.$denotes the unitalization of $\mathcal{A}$ ) are $\Gamma$-invariant idempotents such that $[e]-\left[e^{\prime}\right] \in K_{0}^{\Gamma}(\mathcal{A})$, then

$$
\tau \sharp \operatorname{Tr}(e, \ldots, e \mid h)-\tau \sharp \operatorname{Tr}\left(e^{\prime}, \ldots, e^{\prime} \mid h\right) \in \mathbb{C}
$$

only depends on the class $[e]-\left[e^{\prime}\right]$ in $K$-theory (the cocycle has been trivially extended on the unitalized algebra).

Proof. We will only sketch the proof for the sake of completeness. More details can be found in [7].

The first item follows by $\Gamma$-invariance of the cocycle and the idempotent. In fact, one has

$$
(\tau \sharp T r)\left(e, \ldots, e \mid g h g^{-1}\right)=(\tau \sharp T r)\left(g^{-1} e, g^{-1} e, \ldots, g^{-1} e \mid h\right)=(\tau \sharp T r)(e, \ldots, e \mid h)
$$

which shows that the function $\langle e, \tau\rangle$ is central. Continuity follows immediately from the definition of $\langle e, \tau\rangle$.

Let us now sketch 2). First, one can assume that $\mathcal{A}$ is unital. Moreover, replacing $\mathcal{A}$ by the matrix algebra $M_{2}(\mathcal{A})$, one sees that there exists a $\Gamma$-invariant element $u$ that conjugates the idempotents $e$ and $e^{\prime}$, if they are equivalent. See [17, pag. 229 for details. Thus, the proof is reduced to show that if $e^{\prime}=u e u^{-1}$, then $\langle e, \tau\rangle=\left\langle e^{\prime}, \tau\right\rangle$.

The crucial point of the proof is that, if $e^{\prime}=u e u^{-1}$, there exists an equivariant odd cyclic cochain $\psi_{u}$ such that

$$
\left\langle e^{\prime}, \tau\right\rangle-\langle e, \tau\rangle=\left\langle e, b_{\Gamma}\left(\psi_{u}\right)\right\rangle \text {. }
$$

The explicit construction of the cochain $\psi_{u}$ can be found in [7], Proposition 3.5.

Now, since $\psi_{u}$ is odd and $e$ is $\Gamma$-invariant, one gets

$$
\left\langle e, b_{\Gamma}\left(\psi_{u}\right)\right\rangle(h)=b^{\prime} \psi_{u}(e, \ldots, e \mid h)+\psi_{u}\left(h^{-1} e, e, \ldots, e \mid h\right)=\psi_{u}(e, \ldots, e \mid h) .
$$

Using cyclicity, one immediately deduces that $\psi_{u}(e, \ldots, e \mid h)=0$, which proves the claim.

Remark 3.6. Since by the Peter-Weyl theorem $R[\Gamma] \otimes_{\mathbb{Z}} \mathbb{C}$ is a dense subalgebra of $C(\Gamma)^{\Gamma}$, the pairing 3.48 can be seen as a generalization of the "classical" character-index $i n d^{\Gamma}: K_{0}^{\Gamma}\left(T^{*} M\right) \rightarrow R[\Gamma]$ of a compact $\Gamma$-manifold $M$, due to Atiyah and Singer. For details, we recommend the original papers [4] and [3]. 
Given any equivariant even cyclic cocycle $\tau$ of $(\mathcal{A}, \Gamma)$, consider the additive map

$$
\chi_{\tau}=\langle\cdot, \tau\rangle: K_{0}^{\Gamma}(\mathcal{A}) \rightarrow C(\Gamma)^{\Gamma}
$$

Now, composing the above map with the evaluation $e v_{h}$ at an arbitrary element $h \in \Gamma$, one gets a $\mathbb{C}$-valued additive map

$$
\chi_{\tau}(h)=\langle\cdot, \tau\rangle_{h}=e v_{h} \circ\langle\cdot, \tau\rangle: K_{0}^{\Gamma}(\mathcal{A}) \rightarrow \mathbb{C}
$$

In the case of a foliated $\Gamma$-manifold, one is interested to compute in topological and geometrical terms the scalars $\left\langle[e]-\left[e^{\prime}\right], \tau\right\rangle_{h}$, for classes $[e]-\left[e^{\prime}\right] \in K_{0}^{\Gamma}(\mathcal{A})$ defined in geometric terms, in order to get informations about the foliation and the foliated diffeomorphism $h$.

Notice that evaluating $\langle\cdot, \tau\rangle$ at $h$ or at its conjugacy class $[h]$ yields the same result, since $\langle\cdot, \tau\rangle$ is central. If we want to emphasize this invariance property, we will denote the evaluation $\chi_{\tau}(h)=\langle\cdot, \tau\rangle_{h}$ also by $\chi_{\tau}([h])=\langle\cdot, \tau\rangle_{[h]}$.

Using naturality of the equivariant $K$-groups with respect to subgroups, it follows that the evaluation does not depend upon the group $\Gamma$, as far as this exists. More precisely, if $\Gamma_{1} \leq \Gamma$ is a compact subgroup, let $\operatorname{res}_{\Gamma_{1}}: K_{0}^{\Gamma}(\mathcal{A}) \rightarrow K_{0}^{\Gamma_{1}}(\mathcal{A})$ be the natural forgetful map from $\Gamma$-equivariant $K$-theory to $\Gamma_{1}$-equivariant $K$-theory. Moreover, given an equivariant cyclic $n$-cocycle $\tau$ denote by $R_{\Gamma_{1}}(\tau)$ the restriction of $\tau$ to the subgroup $\Gamma_{1}$. One has the following proposition.

Proposition 3.4.4. Let $\Gamma_{1} \leq \Gamma$ be a compact subgroup.

1. For any equivariant cyclic cocycle $\tau$ on $(\mathcal{A}, \Gamma)$, the diagram

$$
\begin{array}{ccc}
K_{0}^{\Gamma}(\mathcal{A}) & \stackrel{r e s_{\Gamma_{1}}}{\longrightarrow} & K_{0}^{\Gamma_{1}}(\mathcal{A}) \\
\chi_{\tau} \downarrow & & \downarrow \chi_{R_{\Gamma_{1}}(\tau)} \\
C(\Gamma)^{\Gamma} & \stackrel{r}{\rightarrow} & C\left(\Gamma_{1}\right)^{\Gamma_{1}}
\end{array}
$$

is commutative. Here $r: C(\Gamma)^{\Gamma} \rightarrow C\left(\Gamma_{1}\right)^{\Gamma_{1}}$ denotes the restriction map to $\Gamma_{1}$.

2. If $\mathcal{A}$ is a Fréchet $\Gamma$-algebra, there is a well-defined pairing

$$
K_{0}^{\Gamma}(\mathcal{A}) \times H C^{e v}\left(\mathcal{A}, \Gamma_{1}\right) \rightarrow C\left(\Gamma_{1}\right)^{\Gamma_{1}}
$$

defined by the same formula of Equation 3.48, for all $h \in \Gamma_{1}$.

Proof. 1). Let $x=[e]-\left[e^{\prime}\right] \in K_{0}^{\Gamma}(\mathcal{A})$ and let $\operatorname{res}_{\Gamma_{1}}(x)$ be the induced class in $K_{0}^{\Gamma_{1}}(\mathcal{A})$. Then $\operatorname{res}_{\Gamma_{1}}(x)$ can be represented by the idempotents $e$ and $e^{\prime}$. Moreover, consider the restriction $R_{\Gamma_{1}}(\tau)$ of $\tau$ to $\Gamma_{1}$. Then for all $h_{1} \in \Gamma_{1}$ it follows that

$$
\langle e, \tau\rangle\left(h_{1}\right)-\left\langle e^{\prime}, \tau\right\rangle\left(h_{1}\right)=\left\langle e, R_{\Gamma_{1}}(\tau)\right\rangle\left(h_{1}\right)-\left\langle e^{\prime}, R_{\Gamma_{1}}(\tau)\right\rangle\left(h_{1}\right)
$$

where $e$ and $e^{\prime}$ represent $x \in K_{0}^{\Gamma}(\mathcal{A})$ on the LHS and represent $r e s_{\Gamma_{1}}(x)$ on the RHS. Now, since $\Gamma_{1}$ is compact, 1$)$ follows. 
$2)$. Given any $\Gamma_{1}$-equivariant even cyclic cocycle $\tau_{1}$ and any $\Gamma$-invariant idempotent $e \in \mathcal{A} \otimes \operatorname{End}(V)$, where $V$ is a representation of $\Gamma$, it is obvious that the pairing 3.48 makes sense for all $h_{1} \in \Gamma_{1}$ and it does only depend on the cohomology class $\left[\tau_{1}\right] \in H C^{e v}\left(\mathcal{A}, \Gamma_{1}\right)$. Let us pick now another $\Gamma$-invariant idempotent $e^{\prime} \in \mathcal{A} \times \operatorname{End}(V)$ conjugated to $e$ through the $\Gamma$-invariant invertible element $u$. By arguing as in Theorem 3.4.3, there exists a $\Gamma_{1}$-equivariant odd cyclic cochain $\phi_{u}$ such that

$$
\left\langle e, \tau_{1}\right\rangle\left(h_{1}\right)-\left\langle e^{\prime}, \tau_{1}\right\rangle\left(h_{1}\right)=\left\langle e, b_{\Gamma_{1}} \phi_{u}\right\rangle\left(h_{1}\right)
$$

for all $h_{1} \in \Gamma_{1}$. Now, by using the fact that $e$ is in particular $\Gamma_{1}$-invariant and $\phi_{u}$ is odd, one gets that $\left\langle e, b_{\Gamma_{1}} \phi_{u}\right\rangle\left(h_{1}\right)=0$, for every $h_{1} \in \Gamma_{1}$. This proves the claim.

As a consequence of Proposition 3.4.4 if $x \in K_{0}^{\Gamma}(\mathcal{A})$, one can use any subgroup of $\Gamma$ containing $h$ to compute the number $\chi_{\tau}(h)(x)=\langle x, \tau\rangle_{[h]}$. In particular, the proposition implies that the problem of studying the map

$$
\chi_{\tau}(h): K_{0}^{\Gamma}(\mathcal{A}) \rightarrow \mathbb{C}
$$

can be reduced to the case where $\Gamma$ is the topologically cyclic group generated by the element $h$. Thus, as in the classical case, the group $\Gamma$ has been eliminated from the problem.

Another important property of the map 3.53 is that it can be localized at the prime ideal $I_{[h]}$ associated with the conjugacy class $[h]$ of $h \in \Gamma$. Indeed, let us recall that $I_{[h]}$ is the prime ideal of all characters $\rho \in R[\Gamma]$ which vanish in $h$. The equivariant $K$-group $K_{0}^{\Gamma}(\mathcal{A})$ has a $R[\Gamma]$-module structure (see 2.2.1) and therefore it can be localized at $I_{[h]}$, giving rise to the $R[\Gamma]_{I_{[h]}}$-module of fractions $K_{0}^{\Gamma}(\mathcal{A})_{[h]}$. For the notation and further details we refer to Section 2.2.3. Now, one has the following result.

Proposition 3.4.5. With the notation above, for any equivariant even cyclic cocycle $\tau$ of the pair $(\mathcal{A}, \Gamma)$, the additive map

$$
\chi_{\tau}([h])=\langle\cdot, \tau\rangle_{[h]}: K_{0}^{\Gamma}(\mathcal{A}) \rightarrow \mathbb{C}
$$

satisfies

$$
\langle x \rho, \tau\rangle_{[h]}=\langle x, \tau\rangle_{[h]} \cdot \rho(h)
$$

for all $x \in K_{0}^{\Gamma}(\mathcal{A}), \rho \in R[\Gamma]$.

Thus, $\chi_{\tau}([h])$ induces a $R[\Gamma]_{I_{[h]}}$-linear map

$$
\langle\cdot, \tau\rangle_{I_{[h]}}: K_{0}^{\Gamma}(\mathcal{A})_{[h]} \rightarrow \mathbb{C}
$$

such that the diagram

$$
\begin{array}{ccc}
K_{0}^{\Gamma}(\mathcal{A}) & \stackrel{\chi_{\tau}([h])}{\longrightarrow} & \mathbb{C} \\
\downarrow & & \| \\
K_{0}^{\Gamma}(\mathcal{A})_{[h]} & \stackrel{\langle\cdot, \tau\rangle_{[h]}}{\longrightarrow} & \mathbb{C}
\end{array}
$$

is commutative. Here the vertical map $K_{0}^{\Gamma}(\mathcal{A}) \rightarrow K_{0}^{\Gamma}(\mathcal{A})_{[h]}$ is defined by sending $x$ to $\frac{x}{1_{R[\Gamma]}}$. 
Proof. Take two $\Gamma$-invariant idempotents $e \in \mathcal{A}^{+} \otimes \operatorname{End}(V)$ and $e^{\prime} \in \mathcal{A}^{+} \otimes$ $\operatorname{End}\left(V^{\prime}\right)$ such that $[e]-\left[e^{\prime}\right] \in K_{0}^{\Gamma}(\mathcal{A})$. Moreover, let us choose another finite dimensional unitary representation $(W, \Lambda)$ that represents the element $\rho \in R[\Gamma]$. The $K^{\Gamma}$-theory class $x \rho$ is by definition (see Section 2.2.1 the formal difference

$$
x \rho=\left[e \otimes I d_{W}\right]-\left[e^{\prime} \otimes I d_{W}\right]
$$

Now, for any equivariant cyclic $2 k-\operatorname{cocycle} \tau$, one has

$$
\begin{gathered}
\langle x \rho, \tau\rangle_{[h]}=\left(\tau \sharp\left(T r_{V} \sharp T r_{W}\right)\right)\left(e \otimes I d_{W}, \ldots, e \otimes I d_{W} \mid h\right)- \\
-\left(\tau \sharp\left(T r_{V^{\prime}} \sharp T r_{W}\right)\right)\left(e^{\prime} \otimes I d_{W}, \ldots, e^{\prime} \otimes I d_{W} \mid h\right)= \\
=\left(\left(\tau \sharp T r_{V}\right) \sharp T r_{W}\right)\left(e \otimes I d_{W}, \ldots, e \otimes I d_{W} \mid h\right)- \\
-\left(\left(\tau \sharp T r_{V^{\prime}}\right) \sharp T r_{W}\right)\left(e^{\prime} \otimes I d_{W}, \ldots, e^{\prime} \otimes I d_{W} \mid h\right)= \\
\left(\tau \sharp T r_{V}\right)(e, \ldots, e \mid h) \cdot \operatorname{Tr}_{W}(\Lambda(h))-\left(\tau \sharp T r_{V}^{\prime}\right)\left(e^{\prime}, \ldots, e^{\prime} \mid h\right) \cdot \operatorname{Tr}_{W}(\Lambda(h))= \\
=\langle x, \tau\rangle_{[h]} \cdot \rho(h),
\end{gathered}
$$

which proves the desired formula. As a corollary of this and by functoriality of the localization procedure, we get the well defined map on the localized $K$-group

$$
\langle\cdot, \tau\rangle_{[[h]}: K_{0}^{\Gamma}(\mathcal{A})_{[h]} \rightarrow \mathbb{C}
$$

defined by

$$
\left\langle\frac{x}{\rho}, \tau\right\rangle_{[h]}=\frac{\langle x, \tau\rangle_{[h]}}{\rho(h)}
$$

for every $x \in K_{0}^{\Gamma}(\mathcal{A}), \rho \in R[\Gamma] \backslash I_{[h]}$. The commutativity of the diagram follows directly from the above definition.

This proposition is important and will allow us to define higher Lefschetz invariants associated to a foliated $\Gamma$-manifold.

\subsection{Twisted cyclic cohomology and the pairing with equivariant K-theory}

We consider in this paragraph the twisted cyclic cohomology groups of a pair $(\mathcal{A}, \phi)$, with $\mathcal{A}$ a $\mathbb{C}$-algebra and $\phi$ an algebra automorphism of $\mathcal{A}$, and study some of their basic properties.

One motivation to introduce the twisted theory relies on the fact that an equivariant cyclic cocycle on the $\Gamma$-algebra $\mathcal{A}$ may be thought of as a "family" of twisted cyclic cocycles, parametrized in a continuous manner by $\Gamma$. Hence it is natural to consider the elements of this family in their own right and study their basic features. In particular, an easy but interesting fact about twisted cocycles is that it is possible to pair them with equivariant $K$-theory classes, getting additive maps on the equivariant $K$-groups with values in $\mathbb{C}$. This is an immediate consequence of the proof of Theorem 3.4.3 
Another reason to look at the twisted case is that it may be thought of as a special case of the equivariant theory, where one allows actions of the infinite cyclic group $\mathbb{Z}$ generated by the automorphism $\phi$. Even if this group is no longer compact, twisted cohomology turns out to be an interesting theory which enjoys all the properties satisfied by an equivariant theory with respect to a compact group. In particular, we will establish a $S B I$-sequence in the twisted case.

Moreover, in practice one also encounters twisted cyclic cocycles along with equivariant ones, as we will see in Chapter 4

Our treatment of twisted cyclic cohomology will be similar to the treatment we have given for the equivariant theory, showing the formal analogies between the theories. However, the twisted theory can be more clearly formulated in purely algebraic terms for pairs $(\mathcal{A}, \phi)$, given by an algebra and an automorphism of the algebra, without assuming for now any topology on $\mathcal{A}$.

Thus, let $(\mathcal{A}, \phi)$ be a pair consisting of a $\mathbb{C}$-algebra $\mathcal{A}$ and an algebra automorphism $\phi: \mathcal{A} \rightarrow \mathcal{A}$. We will first assume that $\mathcal{A}$ is unital and then we will study how to get rid of this assumption.

Let $C^{n}(\mathcal{A})$ be the space of all $(n+1)$-linear maps on $\mathcal{A} \times \cdots \times \mathcal{A}$ with values in $\mathbb{C}$ and define the corresponding graded vector space $C^{*}(\mathcal{A})=\oplus_{n \geq 0} C^{n}(\mathcal{A})$.

Definition 3.14. Let $\sigma \in C^{n}(\mathcal{A})$. We will call $\sigma$ a twisted cochain if

$$
\sigma\left(\phi\left(a_{0}\right), \phi\left(a_{1}\right), \ldots, \phi\left(a_{n}\right)\right)=\sigma\left(a_{0}, a_{1}, \ldots, a_{n}\right),
$$

for all $a_{i} \in \mathcal{A}$. If one needs to specify the automorphism, we will also call $\sigma$ a $\phi$-twisted cochain.

The vector space of all twisted cochains will be denoted by $C^{n}(\mathcal{A}, \phi)$.

We set

$$
C^{*}(\mathcal{A}, \phi)=\bigoplus_{n \geq 0} C^{n}(\mathcal{A}, \phi)
$$

As in the equivariant case, we define a representation of the cyclic group $\mathbb{Z} /(n+$ $1) \mathbb{Z}$ on $C^{n}(\mathcal{A}, \phi)$ by means of an operator which suitably generalizes the cyclic operator of the ordinary cyclic theory and keeps track of the twisting automorphism $\phi$.

Definition 3.15. The twisted cyclic operator is the operator $\lambda_{\phi}: C^{*}(\mathcal{A}, \phi) \rightarrow$ $C^{*}(\mathcal{A}, \phi)$ of degree zero, defined by setting

$$
\left(\lambda_{\phi} \tau\right)\left(a_{0}, a_{1}, \ldots, a_{n}\right)=(-1)^{n} \tau\left(\phi\left(a_{n}\right), a_{0}, \ldots, a_{n+1}\right)
$$

for all $\tau \in C^{n}(\mathcal{A}, \phi), n \geq 0$.

To prove that the twisted cyclic operator $\lambda_{\phi}$ defines indeed a representation of $\mathbb{Z} /(n+1) \mathbb{Z}$ on $C^{n}(\mathcal{A}, \phi)$, one has to check that

$$
\lambda_{\phi}^{n+1}=i d
$$

This follows from the same computation we did in Remark 3.2 
Definition 3.16. A twisted cochain $\sigma \in C^{n}(\mathcal{A}, \phi)$ is said to be cyclic if $\lambda_{\phi}(\sigma)=$ $\sigma$. The vector space of twisted cyclic cochains will be denoted by $C_{\lambda}^{*}(\mathcal{A}, \phi)$.

Let us also define the twisted norm operator as the degree zero operator

$$
N_{\phi}: C^{*}(\mathcal{A}, \phi) \rightarrow C^{*}(\mathcal{A}, \phi)
$$

that on $C^{n}(\mathcal{A}, \phi)$ is given by the formula

$$
N_{\phi}=\sum_{i=0}^{n} \lambda_{\phi}^{i}
$$

Once again, it follows from Equation 3.59 that

$$
\left(i d-\lambda_{\phi}\right) N_{\phi}=N_{\phi}\left(i d-\lambda_{\phi}\right)=0 .
$$

which in turn implies that the sequence

$$
C^{n}(\mathcal{A}, \Gamma) \stackrel{i d-\lambda_{\phi}}{\longrightarrow} C^{n}(\mathcal{A}, \Gamma) \stackrel{N_{\phi}}{\longrightarrow} C^{n}(\mathcal{A}, \Gamma) \stackrel{i d-\lambda_{\phi}}{\longrightarrow} C^{n}(\mathcal{A}, \Gamma) \stackrel{N_{\phi}}{\longrightarrow} \ldots
$$

is a cochain complex.

The first differential in the twisted theory is, as in the ordinary and in the equivariant theory, the operator $b^{\prime}: C^{n}(\mathcal{A}, \phi) \rightarrow C^{n+1}(\mathcal{A}, \phi)$, given by

$$
\left(b^{\prime} \sigma\right)\left(a_{0}, a_{1}, \ldots, a_{n+1}\right)=\sum_{i=0}^{n}(-1)^{i} \sigma\left(a_{0}, a_{1}, \ldots, a_{i} a_{i+1}, \ldots, a_{n+1}\right)
$$

for all $\sigma \in C^{n}(\mathcal{A}, \phi)$. It is straightforward to see that $C^{*}(\mathcal{A}, \phi)$ is invariant for $b^{\prime}$.

Since this operator does not involve the automorphism $\phi$, one has that $\left(b^{\prime}\right)^{2}=0$, i.e. it is a differential on the twisted cochains. However, the resulting cochain complex $\left(C^{*}(\mathcal{A}, \phi), b^{\prime}\right)$ does not contain any interesting cohomological information, being an acyclic complex. Indeed, since $\mathcal{A}$ is unital, there exist chain contractions for $\left(C^{*}(\mathcal{A}, \phi), b^{\prime}\right)$ defined as in 3.10 .

The twisted Hochschild differential is defined by adding to $b^{\prime}$ an additional term, which takes into account the automorphism. Thus, similarly to the equivariant case, we set

$$
\left(d_{\phi} \sigma\right)\left(a_{0}, a_{1}, \ldots, a_{n+1}\right)=(-1)^{n+1} \sigma\left(\phi\left(a_{n+1}\right) a_{0}, a_{1}, \ldots, a_{n}\right)
$$

and define the twisted Hochschild coboundary map to be

$$
b_{\phi}=b^{\prime}+d_{\phi}: C^{*}(\mathcal{A}, \phi) \rightarrow C^{*+1}(\mathcal{A}, \phi)
$$

As in the proof of Lemma 3.1.2 it follows from $\left(b^{\prime}\right)^{2}=0$ and the relation $d_{\phi} b^{\prime}+b^{\prime} d_{\phi}+\left(d_{\phi}\right)^{2}=0$, that $b_{\phi}^{2}=0$.

Definition 3.17. For any pair $(\mathcal{A}, \phi)$, with $\mathcal{A}$ unital, the twisted Hochschild cohomology of $(\mathcal{A}, \phi)$, denoted by $H H^{*}(\mathcal{A}, \phi)$, is the homology of the cochain complex $\left(C^{*}(\mathcal{A}, \phi), b_{\phi}\right)$. 
For nonunital algebras the previous definition of twisted Hochschild cohomology is not the correct one. To generalize it also in the nonunital case, we will construct a bicomplex, exactly in the same way we constructed the cyclic bicomplex $C C^{* *}(\mathcal{A}, \Gamma)$. We will see the details of this construction in a moment. Before of that, though, let us state the following analogue of Lemma 3.1.3

Lemma 3.5.1. One has the following relations

$$
b^{\prime}\left(i d-\lambda_{\phi}\right)=\left(i d-\lambda_{\phi}\right) b_{\phi}
$$

and

$$
N_{\phi} b^{\prime}=b_{\phi} N_{\phi}
$$

Proof. The proof of Lemma 3.1.3 carries over verbatim in the twisted theory.

Recall that a twisted cyclic cochain is a twisted cochain $\sigma$ such that $(i d-$ $\left.\lambda_{\phi}\right)(\sigma)=0$.

Hence Equation 3.66 implies that

$$
b_{\phi}\left(C_{\lambda}^{*}(\mathcal{A}, \phi)\right) \subseteq C_{\lambda}^{*}(\mathcal{A}, \phi)
$$

and one gets the subcomplex $\left(C_{\lambda}^{*}(\mathcal{A}, \phi), b_{\phi}\right)$. For any pair $(\mathcal{A}, \phi)$, with $\mathcal{A}$ unital, we denote

$$
H_{\lambda}^{n}(\mathcal{A}, \phi)=H_{*}\left(C_{\lambda}^{n}(\mathcal{A}, \phi), b_{\phi}\right) .
$$

So far we assumed the algebra $\mathcal{A}$ to be unital. To get rid of this assumption, one proceeds as we did for the equivariant theory, namely defining a bicomplex. Combining together the relations 3.61, 3.66 and 3.67, we get indeed the following first quadrant bicomplex

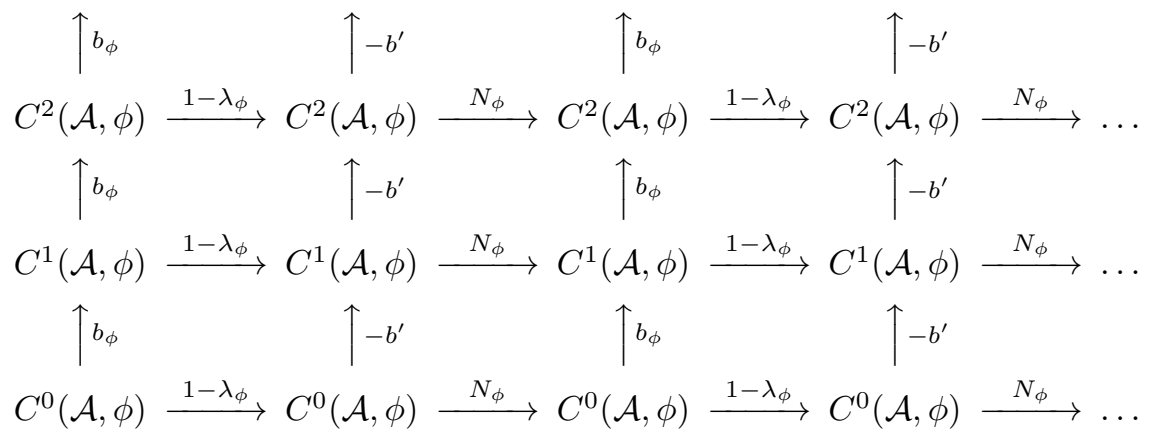

Definition 3.18. The bicomplex above is called the twisted cyclic bicomplex of the pair $(\mathcal{A}, \phi)$ and is denoted by $C C^{* *}(\mathcal{A}, \phi)$. The $n$-dimensional twisted cyclic cohomology group of $(\mathcal{A}, \phi)$ is the $n$-th homology group

$$
H C^{n}(\mathcal{A}, \phi)=H_{n}\left(\operatorname{Tot}^{*}(\mathcal{A}, \phi), \partial_{\phi}\right),
$$

where $\left(\operatorname{Tot}^{*}(\mathcal{A}, \phi), \partial_{\phi}\right)$ is the total complex of $C C^{* *}(\mathcal{A}, \phi)$ such that $\operatorname{Tot}^{n}(\mathcal{A}, \phi)=$ $\bigoplus_{k=0}^{n} C^{n-k}(\mathcal{A}, \phi)$. 
In the definition of $H C^{n}(\mathcal{A}, \phi)$ we do not assume the algebra to be unital. Moreover, the twisted cyclic bicomplex provides a good framework to generalize twisted Hochschild cohomology to nonunital algebras as well.

As for the equivariant theory, see 3.24 set $C_{[2]}^{* *}(\mathcal{A}, \phi)$ to be the bicomplex obtained from $C C^{* *}(\mathcal{A}, \phi)$ by restricting to the first two columns and denote by $\operatorname{Tot}_{[2]}^{*}(\mathcal{A}, \phi)$ its total complex. One may thus consider the homology groups $H_{n}\left(\operatorname{Tot}_{[2]}^{*}(\mathcal{A}, \phi)\right)$, for all $n \in \mathbb{N}$.

If $\mathcal{A}$ has a unit, then the second column of $C_{[2]}^{* *}(\mathcal{A}, \phi)$ does not contribute to the homology of $\operatorname{Tot}_{[2]}^{*}(\mathcal{A}, \phi)$. Hence, in this case,

$$
H_{n}\left(\operatorname{Tot}_{[2]}^{*}(\mathcal{A}, \phi)\right) \cong H_{n}\left(C^{*}(\mathcal{A}, \phi), b_{\phi}\right) \cong H H^{n}(\mathcal{A}, \phi),
$$

and we recover the previous definition of twisted Hochschild cohomology of $(\mathcal{A}, \phi)$.

This leads us to define in general the twisted Hochschild cohomology of $(\mathcal{A}, \phi)$, with $\mathcal{A}$ possibly nonunital, by $H H^{*}(\mathcal{A}, \phi)=H_{*}\left(\operatorname{Tot}_{[2]}^{*}(\mathcal{A}, \phi)\right)$.

Moreover, if $\mathcal{A}$ is unital, we get the analogue of Theorem 3.2 .1 .

Theorem 3.5.2. Given the pair $(\mathcal{A}, \phi)$, with $\mathcal{A}$ unital, then for any $n \in \mathbb{N}$ one gets

$$
H C^{n}(\mathcal{A}, \phi) \cong H_{\lambda}^{n}(\mathcal{A}, \phi) .
$$

The proof of Theorem 3.2.1 can be re-written verbatim to prove the above result.

Remark 3.7. As in the equivariant case, we can compute the twisted cyclic cohomology of a pair $(\mathcal{A}, \phi)$ through the total complex $\left(\operatorname{Tot}^{*}(\mathcal{A}, \Gamma), \partial_{\phi}\right)$ of $C C^{* *}(\mathcal{A}, \phi)$, or alternativily through the $E^{1}$-term of the spectral sequence induced by the twisted bicomplex. The latter complex turns out to be the complex $\left(C_{\lambda}^{*}(\mathcal{A}, \phi), b_{\phi}\right)$ defined exactly as for a unital algebra.

Now, also in the twisted theory as in the equivariant one, one gets easily from the twisted cyclic bicomplex a "SBI-sequence" relating twisted Hochschild cohomology with twisted cyclic cohomology.

We define, for all $\left(x_{0}, x_{1}, \ldots, x_{n}\right) \in \operatorname{Tot}^{n}(\mathcal{A}, \phi)$, the degree 2 cochain map $S_{\phi}$ : $\operatorname{Tot}^{*}(\mathcal{A}, \phi) \rightarrow \operatorname{Tot}^{*}(\mathcal{A}, \phi)$ by

$$
S_{\phi}\left(x_{0}, x_{1}, \ldots, x_{n}\right)=\left(0,0, x_{0}, x_{1}, \ldots, x_{n}\right)
$$

and the projection $I: \operatorname{Tot}^{*}(\mathcal{A}, \phi) \rightarrow \operatorname{Tot}_{[2]}^{*}(\mathcal{A}, \phi)$ by the formula

$$
I\left(x_{0}, x_{1}, \ldots, x_{n}\right)=\left(x_{0}, x_{1}\right),
$$

where again $I\left(x_{0}\right)=x_{0}$, for every $x_{0} \in \operatorname{Tot}^{0}(\mathcal{A}, \Gamma)$.

This gives a short exact sequence of cochain complexes

$$
0 \rightarrow \operatorname{Tot}^{*}(\mathcal{A}, \phi) \stackrel{S_{\phi}}{\longrightarrow} \operatorname{Tot}^{*}(\mathcal{A}, \phi) \stackrel{I}{\rightarrow} \operatorname{Tot}_{[2]}^{*}(\mathcal{A}, \phi) \rightarrow 0,
$$

which, by applying the trick explained in Section 3.3. induces the long exact sequence in cohomology

$$
\cdots \rightarrow H C^{n-2}(\mathcal{A}, \phi) \stackrel{S_{\phi}}{\longrightarrow} H C^{n}(\mathcal{A}, \phi) \stackrel{I}{\rightarrow} H H^{n}(\mathcal{A}, \phi) \stackrel{B_{\phi}}{\longrightarrow} H C^{n-1}(\mathcal{A}, \phi)
$$




$$
\stackrel{S_{\phi}}{\longrightarrow} H C^{n+1}(\mathcal{A}, \phi) \stackrel{I}{\rightarrow} H H^{n+1}(\mathcal{A}, \phi) \stackrel{B_{\phi}}{\longrightarrow} H C^{n}(\mathcal{A}, \phi) \rightarrow \ldots
$$

where $B_{\phi}$ is the boundary homomorphism. This is the twisted $S B I$-sequence. Moreover, by means of the periodicity map $S_{\phi}: H C^{n-2}(\mathcal{A}, \phi) \rightarrow H C^{n}(\mathcal{A}, \phi)$, we define the twisted periodic cyclic cohomology $H P^{n}(\mathcal{A}, \phi)$, with $n=0,1$, to be

$$
H P^{n}(\mathcal{A}, \phi)=\underset{S_{\phi}}{\lim } H C^{n+2 l}(\mathcal{A}, \phi) .
$$

Now, let $(\mathcal{A}, \Gamma, \rho)$ be a locally convex $\Gamma$-algebra, with $\Gamma$ a compact group, and let us fix, once and for all, an element $h \in \Gamma$.

Then one gets the pair $(\mathcal{A}, h)$, where $h$ is viewed as the corresponding continuous automorphism induced by the action $\rho_{h}: \mathcal{A} \rightarrow \mathcal{A}$. Hence one may consider the twisted cyclic cohomology $H C^{*}(\mathcal{A}, h)$.

For any equivariant cyclic cocycle $\tau$ on $\mathcal{A}$, we define a twisted cyclic cocycle $\tau_{h}$ of $(\mathcal{A}, h)$ by simply setting $\tau_{h}=e v_{h^{-1}} \circ \tau$.

Now, an interesting fact is that there is a well defined pairing

$$
K_{0}^{\Gamma}(\mathcal{A}) \times H C^{e v}(\mathcal{A}, h) \rightarrow \mathbb{C} .
$$

between equivariant $K$-theory and twisted cyclic cohomology. Indeed, let $\sigma$ be a $h$-twisted $2 k$-dimensional cochain on the pair $(\mathcal{A}, h)$. For any unitary finite dimensional representation $\Lambda: \Gamma \rightarrow \operatorname{End}(V)$, let us consider the pair $(\mathcal{A} \otimes \operatorname{End}(V), h)$ and set

$\left(\sigma \sharp T r_{h}\right)\left(a^{0} \otimes A^{0}, a^{1} \otimes A^{1}, \ldots, a^{2 k} \otimes A^{2 k}\right)=\sigma\left(a^{0}, a^{1}, \ldots, a^{2} k\right) \operatorname{Tr}_{h}\left(\Lambda(h) A^{0} \cdots A^{2 k}\right)$

extended on the whole algebra by linearity. It is obvious that $\sigma \sharp T r_{h}$ is again a $h$-twisted cochain. Furthermore,

Lemma 3.5.3. 1. If $\sigma \in C_{\lambda}(\mathcal{A}, h)$, then $\sigma \sharp T r_{h} \in C_{\lambda}(\mathcal{A} \otimes \operatorname{End}(V), h)$.

2. One has that $b_{h}\left(\sigma \sharp T r_{h}\right)=\left(b_{h} \sigma\right) \sharp T r_{h}$.

Proof. It follows by a straightforward computation.

Now, similarly to the equivariant case, we set for any $\Gamma$-invariant idempotent $e \in \mathcal{A} \otimes \operatorname{End}(V)$

$$
\langle e, \sigma\rangle_{h}=\left(\sigma_{h} \sharp T r_{h}\right)(e, e, \ldots, e) .
$$

Theorem 3.5.4. 1. The Equation 3.76 induces a well defined pairing

$$
K_{0}^{\Gamma}(\mathcal{A}) \times H C^{e v}(\mathcal{A}, h) \rightarrow \mathbb{C} .
$$

2. The following diagram

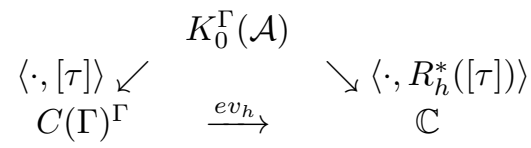

is commutative. 
Proof. 1). To show that the pairing is well-defined one proceeds as in the proof of Theorem 3.4.3 2). More precisely, if two $\Gamma$-invariant idempotents $e, e^{\prime}$ are conjugated by a $\Gamma$-invariant invertible element $u$, i.e. $e^{\prime}=u e u^{-1}$, then one constructs a twisted odd cyclic cochain $\theta_{u}$ such that

$$
\left\langle e^{\prime}, \sigma\right\rangle_{h}-\langle e, \sigma\rangle_{h}=\left\langle e, b_{\phi}\left(\theta_{u}\right)\right\rangle_{h}
$$

Now, being $\theta_{u}$ odd and $e \Gamma$-invariant, one easily deduces that $\left\langle e, b_{\phi}\left(\theta_{u}\right)\right\rangle_{h}=0$. Finally, it is immediate to see that two even twisted cocycles that are cohomologous determine the same map on equivariant $K$-theory.

2). It is straightforward.

In other words, any even cyclic cocycle $\sigma$ twisted by a fixed $h \in \Gamma$ yields an additive map

$$
\left\langle\cdot, \sigma_{h}\right\rangle: K_{0}^{\Gamma}(\mathcal{A}) \rightarrow \mathbb{C}
$$

which does depend only on the cyclic cohomology class of $\sigma_{h}$. 


\section{Chapter 4}

\section{Equivariant Cyclic Cocycles for foliated manifolds}

In this chapter we are going to introduce and study important equivariant cyclic cocycles on a foliated $\Gamma$-manifold $(M, \mathcal{F}, \Gamma)$.

One of the interesting facts is that some of the geometric cyclic cocycles on the foliated manifold are actually seen to be the evaluation at the identity of some equivariant cyclic cocycles on $(M, \mathcal{F}, \Gamma)$. Indeed, under some nontrivial assumptions on the action of $\Gamma$ on $(M, \mathcal{F})$ Benameur and Heitsch proved in 7 that, for instance, a transverse fundamental cyclic cocycle $\tau$, representing the transverse fundamental class on the convolution algebra $\left(C_{c}^{\infty}(H(\mathcal{F}))\right)$, is $\Gamma$-invariant and is just the evaluation at the identity element of an equivariant cyclic cocycle $\varphi$ of $(M, \mathcal{F}, \Gamma)$. Their arguments work more generally for any cyclic cocycle arising from a closed even-dimensional Haefliger current. Therefore each of these cocycles can be thought of as a small part of an equivariant cyclic cocycle, namely the evaluation at the identity of $\Gamma$.

One limitation of their approach is that they have to work with the holonomy groupoid $H(\mathcal{F})$ and therefore consider only holonomy diffeomorphisms (see Def. 1.32 , excluding the general case. Using the twisted holonomy groupoid, we will be able to construct cyclic cocycles equivariant with respect to any diffeomorphism, not only those satisfying the holonomy assumption.

Besides, the equivariant cocycles mentioned above describe characteristic classes of the foliation, which do not exceed by construction the codimension $q$ of $(M, \mathcal{F})$. On the other side, foliations admit "exotic" characteristic classes, which do exceed the codimension. Thus, it is natural to ask whether there exist equivariant cyclic cocycles describing the exotic classes of a foliated $\Gamma$-manifold. In this direction, we will focus on the simplest non trivial example of these secondary characteristic classes, namely the Godbillon-Vey class of a transversely oriented foliated manifold $(M, \mathcal{F})$ of codimension 1.

Using the general theory developed in the previous chapter, and in particular the pairing 3.4 .3 between equivariant K-theory and equivariant cyclic cocycles, we will be able to define higher Lefschetz numbers of leafwise diffeomorphisms 
with respect to invariant leafwise elliptic pseudodifferential operators.

Notation. In the following, unless otherwise specified we shall assume that a foliated manifold is always a closed manifold $M$ endowed with a $C^{\infty}$-foliation $\mathcal{F}$.

Given a vector bundle $E$ over the foliated manifold $(M, \mathcal{F})$ and given any Lie groupoid $G$ integrating $\mathcal{F}$, we will denote the convolution algebra of smooth compactly supported sections on $G$ of the bundle $E N D(E)=s^{*}\left(E^{*}\right) \otimes t^{*}(E)$ over $G$ simply by $C_{c}^{\infty}(G, E)$.

\subsection{The equivariant transverse fundamental class}

Given a transversely oriented foliated $\Gamma$-manifold $(M, \mathcal{F}, \Gamma)$, we will show that if the action of $\Gamma$ preserves the transverse orientation then the transverse fundamental cyclic cocycle is the evaluation at the identity of an equivariant cyclic cocycle.

Our method is inspired by [7]. Nevertheless our setting is more general, since we do not have to assume the diffeomorphisms acting on the foliated manifold to be holonomy diffeomorphisms (see Definition 1.32). Indeed, since the twisted holonomy groupoid $H(\mathcal{F}, \Gamma)$ encodes the transverse action of $\Gamma$ on the foliation, it is natural to construct the equivariant transverse fundamental cyclic cocycle as an equivariant cocycle on the twisted holonomy groupoid. This holds for more general cocycles coming from Haefliger's holonomy invariant currents, as well. However, using the twisted holonomy groupoid it is no longer possible to show that all these cyclic cocycles can be made equivariant, as proved in [7]. In fact one has to require a stronger invariance for the currents, namely also the invariance with respect to the transverse action of $\Gamma$ given by holonomies.

Using these equivariant cyclic cocycles, we will be able to define higher Lefschetz numbers of the foliation for general leaf-preserving diffeomorphisms.

\subsubsection{Haefliger-type transverse cohomologies}

Let $(M, \mathcal{F}, \Gamma)$ be a foliated $\Gamma$-manifold of codimension $q$ and let $H(\mathcal{F}, \Gamma)$ be its twisted holonomy groupoid.

Recall that a complete transversal $T$ is a $q$-dimensional submanifold transverse to the foliation which meets every leaf of the foliation. Without requiring $T$ to be connected or compact, such complete transversals exist always.

Taking, now, any complete transversal $T$, the reduced twisted holonomy groupoid over $T$, i.e.

$$
G=H(\mathcal{F}, \Gamma)_{T}^{T} \rightrightarrows T
$$

is a smooth étale groupoid, since according to Theorem $1.4 .4 H(\mathcal{F}, \Gamma)$ is a foliation groupoid (for further comments about this point, see also [19]). Moreover, this étale groupoid is effective, as we have seen in Theorem 1.4.5.

Now, one can always construct a pseudogroup $P(\mathcal{G})$ out of an étale effective 
groupoid $\mathcal{G}$ by setting

$$
P(\mathcal{G})=\{t \circ \beta \mid \beta \text { is a local bisection of } \mathcal{G}\} .
$$

This is clearly a pseudogroup on the space of objects $\mathcal{G}^{(0)}$ of $\mathcal{G}$. Given a complete transversal $T$, we denote the pseudogroup associated to $G=H(\mathcal{F}, \Gamma)_{T}^{T}$ by $P_{\Gamma}$ or simply by $P$.

With $P_{\Gamma}$ at hand we define a Haefliger cohomology by means of $P_{\Gamma}$-invariant differential forms on $T$.

Let $\Omega_{c}^{p}(T)$ denote the space of $p$-differential forms on $T$. Following [27], we consider the quotient of $\Omega_{c}^{p}(T)$ by the subspace generated by the forms of the form $\omega-h^{*} \omega$, where $h \in P_{\Gamma}$ and the support of $\omega \in \Omega_{c}^{p}(T)$ is contained in the range of $h$. The topology on the quotient space is the quotient topology of the locally convex topology on $\Omega_{c}^{p}(T)$. We will denote this quotient by $\Omega_{c}^{p}(\operatorname{Tr}(\mathcal{F}, \Gamma))$, since $P_{\Gamma}$ is the pseudogroup associated to the reduced twisted holonomy groupoid. Morever, we set $\Omega_{c}^{*}(\operatorname{Tr}(\mathcal{F}, \Gamma))=\bigoplus_{p \geq 0} \Omega_{c}^{p}(\operatorname{Tr}(\mathcal{F}, \Gamma))$.

The definition of the space $\Omega_{c}^{*}(\operatorname{Tr}(\mathcal{F}, \Gamma))$ does not depend on the choice of the complete transversal $T$. Indeed, if $T^{\prime}$ is another complete transversal and $P_{\Gamma}^{\prime}$ is the pseudogroup associated to $H(\mathcal{F}, \Gamma)_{T^{\prime}}^{T^{\prime}}$, then there exists an isomorphism from $P_{\Gamma}$ to $P_{\Gamma}^{\prime}$ (in the sense of [27], Section 1.1), since $H(\mathcal{F}, \Gamma)_{T^{\prime}}^{T^{\prime}}$ and $H(\mathcal{F}, \Gamma)_{T}^{T}$ are Morita equivalent (see [39], Exercise 5.24). This induces a continuous isomorphism between $\Omega_{c}^{p}(T) /<\omega-h^{*} \omega>$ and $\Omega_{c}^{p}\left(T^{\prime}\right) /<\eta-\left(h^{\prime}\right)^{*} \eta>$. We refer to [27, Section 1.2. for details.

The de Rham differential $d: \Omega_{c}^{p}(T) \rightarrow \Omega_{c}^{p+1}(T)$ descends to the quotient $\Omega_{c}^{*}(\operatorname{Tr}(\mathcal{F}, \Gamma))$ giving rise to a cochain complex $\left(\Omega_{c}^{*}(\operatorname{Tr}(\mathcal{F}, \Gamma)), d_{H}\right)$, whose cohomology is denoted by $H^{*}(\operatorname{Tr} \mathcal{F}, \Gamma)$.

A continuous linear form on $\Omega_{c}^{k}(\operatorname{Tr}(\mathcal{F}, \Gamma))$ is a $k$-current $C$ defined on every transversal submanifold which is $P_{\Gamma}$-invariant, i.e. $C(\alpha)=C\left(h^{*} \alpha\right)$, for any $h \in P_{\Gamma}$ and any $\alpha \in \Omega_{c}^{k}(T)$ with support contained in the range of $h$. The space of $P_{\Gamma}$-invariant currents will be denoted by $C_{*}(\operatorname{Tr}(\mathcal{F}, \Gamma))$. These currents will provide interesting graded traces, which we are going to use to construct the equivariant cyclic cocycles we want to define.

Denoting by $\partial_{H}$ the dual of the differential $d_{H}$ one gets the dual chain complex $\left(C_{*}(\operatorname{Tr} \mathcal{F}, \Gamma), \partial_{H}\right)$, whose homology will be denoted by $H_{*}(\operatorname{Tr}(\mathcal{F}, \Gamma))$.

Remark 4.1. Since the holonomy groupoid $H(\mathcal{F})$ is an open subgroupoid of $H(\mathcal{F}, \Gamma)$ it is obvious that any $P_{\Gamma}$-invariant current is in particular holonomy invariant.

Example 4.1. Let $(M, \mathcal{F}, \Gamma)$ be a transversely oriented foliated $\Gamma$-manifold of codimension $q$ and denote by $\nu$ the oriented normal bundle to the foliation.

Let us assume that the induced action of $\Gamma$ on $\nu$ consists of orientation-preserving diffeomorphisms.

Then each element $\gamma \in H(\mathcal{F}, \Gamma), \gamma: x \rightarrow y$, gives rise to an orientationpreserving linear isomorphism $\lambda(\gamma): \nu_{x} \rightarrow \nu_{y}$. Indeed, first recall that to an arbitrary element $\gamma \in H(\mathcal{F}, \Gamma)_{x}^{y}$ one associates the linear isomorphism $\lambda(\gamma)$ : 
$\nu_{x} \rightarrow \nu_{y}$, which is the differential at the point $x$ of the transverse local diffeomorphism determined by $\gamma$. See Theorem 1.4.5 and Corollary 1.4.6 for details. By construction of the twisted holonomy groupoid, each $\lambda(\gamma)$ can be written as the composition of infinitesimal holonomies $\lambda(\alpha)$, with $\alpha \in H(\mathcal{F})$, and of the differential $\mathrm{dHol}(h)$ of the transverse local diffeomorphisms $\mathrm{Hol}(h)$ induced by the elements $h \in \Gamma$. Being the foliation transversely oriented and being the induced action of $\Gamma$ on $\nu$ orientation-preserving by hypothesis, it follows that the linear maps $\lambda(\alpha)$ and $d H o l(h)$, for every $\alpha \in H(\mathcal{F}), h \in \Gamma$, are orientationpreserving. Therefore, $\lambda(\gamma)$ preserves the orientation of $\nu$.

For a given complete transversal $T$ and for any $\omega \in \Omega_{c}^{q}(T)$ one can integrate $\omega$ using the transverse orientation. Moreover, one has $\int_{T} \omega=\int_{T} h^{*} \omega$, whenever this makes sense, since $h \in P_{\Gamma}$ is orientation-preserving. Hence we get the invariant $q$-current

$$
\int: \Omega_{c}^{q}(\operatorname{Tr}(\mathcal{F}, \Gamma)) \rightarrow \mathbb{C} \quad[\omega] \mapsto \int[\omega]
$$

This invariant current is fundamental to construct the transverse fundamental cyclic cocycle associated to a horizontal distribution.

To end this section, we recall the following result, which will be needed later.

Theorem 4.1.1. Let $(M, \mathcal{F}, \Gamma)$ a foliated $\Gamma$-manifold and let $\nu$ denote the normal bundle to the foliation. Moreover let $\Omega_{F}$ be the line bundle of leafwise $1-$ densities on $M$ and consider the bundle $\Omega_{F} \otimes \Lambda^{*} \nu^{*}$ over $M$. Then there exists a continuous open surjective linear map

$$
\int_{\mathcal{F}}: C^{\infty}\left(M, \Omega_{F} \otimes \Lambda^{k} \nu^{*}\right) \rightarrow \Omega_{c}^{k}(\operatorname{Tr}(\mathcal{F}, \Gamma)) .
$$

To define the above integration along the leaves one proceeds as follows. Let us give a regular foliated atlas $\mathcal{U}=\left\{U_{i}\right\}$ with transverse coordinate maps $y_{i}: U_{i} \rightarrow T_{i}$ and consider the disjoint union $T=\amalg_{i} T_{i}$. The submanifold $T$ is clearly a complete transversal to the foliation. Taking a smooth partition of unity subordinated to the open cover $\left\{U_{i}\right\}$ one may express any section $s \in$ $C^{\infty}\left(M, \Omega_{F} \otimes \Lambda^{k} \nu^{*}\right)$ as a finite sum $\sum s_{i}$, where each $s_{i}$ has support contained in $U_{i}$. Moreover, each $s_{i}$ is a leafwise density with transverse differential forms as coefficients. Hence it makes sense to integrate each $s_{i}$ along the leaves to get a $k$-differential form $\int_{U_{i} / T_{i}} s_{i}$ on the transversal $T_{i}$. The Haefliger form $\int_{\mathcal{F}} s$ is then defined to be the class in $\Omega_{c}^{k}(\operatorname{Tr}(\mathcal{F}, \Gamma))$ of the differential $k$-form $\sum \int_{U_{i} / T_{i}} s_{i}$ on the complete transversal $T$. It is easy to verify that this definition does not depend on the choice of the partition of unity nor on the foliated atlas. For details and further comments we refer to [27].

\subsubsection{Cyclic cocycles on the twisted holonomy groupoid}

In this section we will construct geometric cyclic coycles on the smooth convolution algebra of the twisted holonomy groupoid using the Haefliger (co-)homology introduced in the previous section. In particular, we will define the transverse 
fundamental cyclic cocycle associated to a horizontal distribution of a transversely oriented foliated $\Gamma$-manifold.

The procedure adopted here is a general way to construct cyclic cocycles and is taken from [17, [7] and [6].

Let $(M, \mathcal{F}, \Gamma)$ denote as usual a foliated $\Gamma$-manifold and let $F$ and $\nu$ be respectively the tangent and the normal bundles to the foliation. For notational simplicity, in the following we will denote the corresponding complexified bundles with the same symbols. Moreover we will denote by $G$ the twisted holonomy groupoid $H(\mathcal{F}, \Gamma)$ and by $\Omega^{1 / 2}$ the bundle of densities $\Omega^{1 / 2} H(\mathcal{F}, \Gamma)$.

Finally, let $E$ be a Hermitian vector bundle on $M$ endowed with a connection $\nabla$.

We may consider the vector bundle $E N D(E) \otimes t^{*}\left(\Lambda^{*} \nu^{*}\right) \otimes \Omega^{1 / 2}$ over $G$, where $E N D(E)=s^{*}(E)^{*} \otimes t^{*}(E)$. The space of compactly supported smooth sections

$$
\Omega_{E}^{*}=C_{c}^{\infty}\left(G, E N D(E) \otimes t^{*}\left(\Lambda^{*} \nu^{*}\right) \otimes \Omega^{1 / 2}\right)
$$

becomes a graded algebra if endowed with the following product. For $T_{1} \otimes \omega_{1} \in$ $\Omega_{E}^{k}$ and $T_{2} \otimes \omega_{2} \in \Omega_{E}^{m}$ we set

$$
\left(\left(T_{1} \otimes \omega_{1}\right) *\left(T_{2} \otimes \omega_{2}\right)\right)(\gamma)=\int_{\gamma_{1} \gamma_{2}=\gamma}\left(T_{1}\left(\gamma_{1}\right) T_{2}\left(\gamma_{2}\right)\right) \otimes\left(\omega_{1}\left(\gamma_{1}\right) \wedge \lambda_{\gamma_{1}} \omega_{2}\left(\gamma_{2}\right)\right)
$$

where $\lambda_{\gamma_{1}}: \Lambda^{m} \nu_{s\left(\gamma_{1}\right)}^{*} \rightarrow \Lambda^{m} \nu_{t\left(\gamma_{1}\right)}^{*}$ denotes the action of the twisted holonomy groupoid on transverse covectors, and then we extend it by linearity for general tensors.

There is a transverse differentiation $\nabla_{Q}$ on $\Omega_{E}^{*}$ of degree 1 . It does depend on the choice of a horizontal distribution $Q \subseteq T M$, i.e. on a splitting of the short exact sequence $0 \rightarrow F \rightarrow T M \rightarrow \nu \rightarrow 0$.

Let us then fix such a horizontal distribution. The transverse differentiation is constructed as follows. First we need to define it for sections of the bundle $E \otimes \Lambda^{*} F^{*} \otimes \Lambda^{*} Q^{*}$. Notice that the connection $\nabla$ on $E$ induces a differential operator, denoted again by $\nabla$,

$$
\nabla: C^{\infty}\left(M, E \otimes \Lambda^{k} T^{*} M\right) \rightarrow C^{\infty}\left(M, E \otimes \Lambda^{k+1} T^{*} M\right)
$$

Using the isomorphism $\psi: \Lambda^{*} T^{*} M \cong \Lambda^{*} F^{*} \otimes \Lambda^{*} Q^{*}$ provided by the horizontal distribution, we define $\nabla_{Q} \omega$ for any form $\omega \in \Lambda^{r} F^{*} \otimes \Lambda^{s} Q^{*}$ as the $(r, s+$ $1)$-component of $\psi \nabla\left(\psi^{-1} \omega\right)$, getting the operator

$$
\nabla_{Q}: C^{\infty}\left(M, E \otimes \Lambda^{r} F^{*} \otimes \Lambda^{s} \nu^{*}\right) \rightarrow C^{\infty}\left(M, E \otimes \Lambda^{r} F^{*} \otimes \Lambda^{s+1} \nu^{*}\right) .
$$

Next, we need to differentiate in the transverse direction half-densities on $M$ with coefficients in the dual bundle $E^{*}$. We use here the dual connection $\nabla^{*}$ on $E^{*}$, induced canonically by the connection on $E$.

Any half-density $\rho$ can be written as $\rho=f|\lambda|^{1 / 2}$, where $f \in C^{\infty}\left(V, E^{*}\right)$ and $\lambda \in C^{\infty}\left(M, \Omega_{\mathcal{F}}^{1}\right)$. Thus, we set

$$
\nabla_{Q}\left(f|\lambda|^{1 / 2}\right)=\left(\nabla_{Q}^{*} f\right)|\lambda|^{1 / 2}+\frac{1}{2} f|\lambda|^{1 / 2} k(\lambda)
$$


where $k(\lambda) \in C^{\infty}\left(M, Q^{*}\right) \cong C^{\infty}\left(M, \nu^{*}\right)$ is the transverse 1 -form defines as follows. Take $p \in V$ and let us choose foliated coordinates $\left(x_{1}, \ldots, x_{p}, t_{1}, \ldots t_{q}\right) \in$ $I^{p} \times I^{q}$ around $p$. In these coordinates, $p=\left(x_{0}, t_{0}\right)$ and the 1 -density $\lambda$ has the form

$$
\lambda(x, t)=\alpha(x, t)\left|d x_{1} \wedge d x_{2} \wedge \cdots \wedge d x_{p}\right| .
$$

Now, if $X(t)=\sum_{i=1}^{q} X_{i}(t) \frac{\partial}{\partial t_{i}}$ is any vector field on the transversal $I^{q}$ and $X_{Q}$ denotes its unique horizontal lift on $I^{p} \times I^{q}$ given by

$$
X_{Q}(x, t)=\sum_{j=1}^{p} X_{Q}^{j}(x, t) \frac{\partial}{\partial x_{j}}+\sum_{i=1}^{q} X_{i}(t) \frac{\partial}{\partial t_{i}},
$$

then the transverse 1 -form $k(\lambda)$ evaluated at $p=\left(x_{0}, t_{0}\right)$ on the transverse vector $X\left(t_{0}\right) \in \nu_{p}$ is

$$
X_{Q}(f)\left(x_{0}, t_{0}\right)+\sum_{j=1}^{p}\left(\frac{\partial}{\partial x_{j}} X_{Q}^{j}\left(x_{0}, t_{0}\right)\right) f\left(x_{0}, t_{0}\right) .
$$

It can be checked that this definition does not depend on the choice of the local coordinates. We refer to 35, Section 5.3 for further details and comments.

We get therefore the transverse differentiation

$$
\nabla_{Q}: C^{\infty}\left(M, E^{*} \otimes \Omega_{F}^{1 / 2}\right) \rightarrow C^{\infty}\left(M, \nu^{*} \otimes E^{*} \otimes \Omega_{F}^{1 / 2}\right)
$$

Finally we need to differentiate transversely a smooth function $f \in C^{\infty}(G)$. To do this, let us consider $\gamma \in G=H(\mathcal{F}, \Gamma)$ and a normal vector $X_{0} \in \nu_{t(\gamma)}$. Set $X_{1}=h_{\gamma^{-1}} X_{0} \in \nu_{s(\gamma)}$. Next, using the horizontal distribution $Q$ lift these two vectors to the corresponding unique horizontal vectors $X_{0}^{Q}$ and $X_{1}^{Q}$. Since $G$ is a foliation groupoid, the anchor map $(s, t): G \rightarrow M \times M$ is an immersion. Hence, $\left(X_{1}^{Q}, X_{0}^{Q}\right) \in T_{(s(\gamma), t(\gamma))}(M \times M)$ is the image of a unique vector $Y \in T_{\gamma} G$. One defines

$$
\left(d_{Q} f\right)(X)=d f(Y)
$$

where $d$ is the usual de Rham differential on the groupoid $G$.

Now, putting together all these pieces we obtain a graded derivation $\nabla_{Q}$ on $\Omega_{E}^{*}$ of degree 1. Recall first that every element $k \in C_{c}^{\infty}\left(G, E N D(E) \otimes \Omega^{1 / 2}\right)$ can be written as $k=f s^{*}(\alpha) t^{*}(\beta)$, where $f \in C_{c}^{\infty}(G), \alpha \in C_{c}^{\infty}\left(M, E^{*} \otimes \Omega_{\mathcal{F}}^{1 / 2}\right), \beta \in$ $C_{c}^{\infty}\left(M, E \otimes \Omega_{\mathcal{F}}^{1 / 2}\right)$. Then

Lemma 4.1.2. For any $k \in C_{c}^{\infty}\left(G, E N D(E) \otimes \Omega^{1 / 2}\right)$ the formula

$$
\nabla_{Q}\left(f s^{*}(\alpha) t^{*}(\beta)\right)=\left(\nabla_{Q} f\right) s^{*}(\alpha) t^{*}(\beta)+f s^{*}\left(\nabla_{Q} \alpha\right) t^{*}(\beta)+f s^{*}(\alpha) t^{*}\left(\nabla_{Q} \beta\right)
$$

defines a derivation of degree 1 from $\Omega_{E}^{0}$ to $\Omega_{E}^{1}$. This derivation extends in a unique way to a graded derivation on $\Omega_{E}^{*}$.

Remark 4.2. If the foliation is oriented, choosing a positive nowhere halfdensity one can work directly with functions rather than with half-densities. 
Through this identification the graded algebra $\Omega_{E}^{*}$ can be taken as the convolution algebra $C_{c}^{\infty}\left(G, E N D(E) \otimes t^{*}\left(\Lambda^{*}\left(\nu^{*}\right)\right)\right)$. Moreover, one can take as transverse derivation $\nabla_{Q}: C_{c}^{\infty}(G, E N D(E)) \rightarrow C_{c}^{\infty}\left(G, E N D(E) \otimes t^{*}\left(\nu^{*}\right)\right)$ the pullback transverse derivative, i.e. the transverse derivative as defined by Equation 4.9 and by Equation 4.5

The pair $\left(\Omega_{E}^{*}, \nabla_{Q}\right)$ is not a differential graded algebra, since the horizontal distribution is not in general integrable and therefore $\left(\nabla_{Q}\right)^{2}$ may be not zero. Indeed $\left(\nabla_{Q}\right)^{2}$ involves the curvature tensor of the connection $\nabla$ on $E$ and the "curvature" of the horizontal distribution $Q$. More precisely, it is shown in [17, Chapter III, Section 7. $\alpha$, for trivial $E$, and in 24], Section 4.6 in general, that $\left(\nabla_{Q}\right)^{2}$ determines a degree 2 multiplier of $\Omega_{E}^{*}$, i.e. there exists a unique element $\theta \in C_{c}^{-\infty}\left(G, E N D(E) \otimes t^{*} \Lambda^{2} \nu^{*} \otimes \Omega^{1 / 2}\right)$ such that

$$
\left(\nabla_{Q}\right)^{2} \omega=[\theta, \omega]
$$

for all $\omega \in \Omega_{E}^{*}$. See also $[$ ].

Using that $\left(\nabla_{Q}\right)^{2}$ has the above form and following [17], Lemma 9, Chapter 3, Section 3 or [7], we can construct a DGA $\left(\overline{\Omega_{E}^{*}}, \delta\right)$ out of $\left(\Omega_{E}^{*}, \nabla_{Q}\right)$ by setting

- $\overline{\Omega_{E}^{k}}=M_{2}\left(\Omega_{E}^{k}\right)$.

- for any $A=\left(\alpha_{i j}\right), B=\left(\beta_{i j}\right) \in M_{2}\left(\Omega_{E}^{k}\right)$, the product is defined by

$$
A * B=A \Theta B
$$

with $\Theta=\left(\begin{array}{ll}1 & 0 \\ 0 & \theta\end{array}\right)$

- the differential $\delta$ of any element $A=\left(\alpha_{i j}\right) \in M_{2}\left(\Omega_{E}^{k}\right)$ is defined by the formula

$$
\delta(A)=\left(\begin{array}{cc}
\nabla_{Q} \alpha_{11} & \nabla_{Q} \alpha_{12} \\
\nabla_{Q} \alpha_{21} & \nabla_{Q} \alpha_{22}
\end{array}\right)+\left(\begin{array}{cc}
0 & -\theta \\
1 & 0
\end{array}\right) A+(-1)^{\partial A} A\left(\begin{array}{cc}
0 & 1 \\
-\theta & 0
\end{array}\right),
$$

where $\partial A$ is the grading of $A \in M_{2}\left(\Omega_{E}^{k}\right)$ (see [17]). It is straightforward to verify that $\delta^{2}=0$.

The algebra $\Omega_{E}^{*}$ is naturally a subalgebra of $\overline{\Omega_{E}^{*}}$ by using the inclusion

$$
\omega \mapsto\left(\begin{array}{cc}
\omega & 0 \\
0 & 0
\end{array}\right)
$$

Remark 4.3. So far we have not used the fact that $G$ is the twisted holonomy groupoid of a foliated $\Gamma$-manifold. In fact, all the algebras and operators can be defined for any foliation groupoid.

Let us now construct traces on $\Omega_{E}^{*}$, using $P_{\Gamma}$-invariant currents.

Let $C \in C_{k}(\operatorname{Tr}(\mathcal{F}, \Gamma))$ a closed $P_{\Gamma}$-invariant current and let $\omega \in \Omega_{E}^{k}$. The restriction $\omega_{\mid M}$ of $\omega$ to the space of objects $M$ yields a smooth section of the bundle 
$\operatorname{End}(E) \otimes \Lambda^{k} \nu^{*} \otimes \Omega_{F}^{1}$. Moreover we can take pointwise the trace $\operatorname{tr}\left(\omega_{\mid M}\right)(m)$ of $\omega_{\mid M}(m) \in \operatorname{End}\left(E_{m}\right) \otimes \Lambda^{k} \nu_{m}^{*} \otimes\left(\Omega_{F}^{1}\right)_{m}$. This yields of course a section

$$
\operatorname{tr}\left(\omega_{\mid M}\right) \in C^{\infty}\left(M, \Lambda^{k} \nu^{*} \otimes \Omega_{F}^{1}\right) .
$$

Applying the map 4.3 to this section we get

$$
\int_{\mathcal{F}} \operatorname{tr}\left(\omega_{\mid M}\right) \in \Omega_{c}^{k}(\operatorname{Tr}(\mathcal{F}, \Gamma)) .
$$

Thus, we can define

$$
\int_{C}: \Omega_{E}^{*} \rightarrow \mathbb{C}
$$

by posing

$$
\int_{C} \omega=\left\langle\int_{\mathcal{F}} \operatorname{tr}\left(\omega_{\mid M}\right), C\right\rangle \quad\left(\int_{C} \eta=0 \quad \text { if } \quad \operatorname{deg}(\eta) \neq k\right)
$$

Lemma 4.1.3. The linear map $\int_{C}$ defines a closed graded trace on $\Omega_{E}^{*}$.

See [6] for a proof of this result and further comments.

Now the closed graded trace $\int_{C}$ extends to a closed graded trace on $\overline{\Omega_{E}^{*}}$ by setting for any $A=\left(\alpha_{i j}\right) \in \overline{\Omega_{E}^{k}}$

$$
\bar{\int}_{C} A=\int_{C} \alpha_{11}-(-1)^{k} \int_{C} \alpha_{22} \theta
$$

We refer to [17, Lemma 9, pag. 234 for the details about this point. Summarizing, for any Hermitian vector bundle $E$ over $M$ with connection $\nabla$ and any homogeneous closed invariant current $C \in C_{*}(\operatorname{Tr}(\mathcal{F}, \Gamma))$, we have constructed a DGA $\left(\overline{\Omega_{E}^{*}}, \delta\right)$, endowed with a closed graded trace $\overline{\int_{C}}$.

Recall that $C_{c}^{\infty}(H(\mathcal{F}, \Gamma), E)=\Omega_{E}^{0}$ is contained in $\overline{\Omega_{E}^{0}}$ through the inclusion 4.14

One has

Theorem 4.1.4. For any Hermitian vector bundle $E$ over $M$ with connection $\nabla$ and any closed $P_{\Gamma}$-invariant current $C \in C_{k}(\operatorname{Tr}(\mathcal{F}, \Gamma))$ defined on the foliated $\Gamma$-manifold $(M, \mathcal{F}, \Gamma)$, the formula

$$
\tau_{C}\left(a^{0}, a^{1}, \ldots, a^{k}\right)=\int_{C}\left(a^{0} * \delta a^{1} * \cdots * \delta a^{k}\right)_{11}
$$

defines a cyclic $k$-cocycle over $C_{c}^{\infty}(H(\mathcal{F}, \Gamma), E)$, which is continuous with respect to the LF-locally convex topology on $C_{c}^{\infty}(H(\mathcal{F}, \Gamma), E)$.

Before proving the above theorem, let us make some observations. For the sake of simplicity, let us assume in the following that the bundle $E$ is trivial and let us assume to have fixed a smooth positive leafwise density. Thus, we will consider functions, rather than densities. Furthermore, let us denote the groupoid $H(\mathcal{F}, \Gamma)$ simply by $G$. We observe that without fixing a density and for general bundles $E$, the discussion below is completely analogous. 
On the spaces $\Omega^{k}$, for $k=0, \ldots, q$, we have two natural topologies compatible with the vector space structure. The first one is the LF-topology, which turns each $\Omega^{k}$ into a LF-space (cf. [46], pag. 15 and the references therein, or [10], Chapter II, Section 6). If we want to emphasize it, we will denote $\Omega^{k}$ endowed with this topology by the symbol $\Omega_{L F}^{k}$.

The other topology is the so-called compact-open $C^{\infty}$ topology. For the definition of this topology, we refer to [31], Chapter 2. In this case, if $\Omega^{k}$ carries the compact-open $C^{\infty}$ topology, we shall denote it by $\Omega_{C O}^{k}$.

In the proof of the above theorem we will need the following lemma, whose proof can be found in Appendix A.

Lemma 4.1.5. Fix $k \leq q$, where $\operatorname{codim} \mathcal{F}=q$, and consider the space $\Omega_{C O}^{k}$. Then, for any $m$-tuple $\left(k_{1}, k_{2}, \ldots, k_{m}\right) \in \mathbb{N}^{l}$ such that $\sum_{l=0}^{m} k_{l}=k$ the multilinear map

$$
\Omega_{L F}^{k_{1}} \times \Omega_{L F}^{k_{2}} \times \cdots \times \Omega_{L F}^{k_{m}} \rightarrow \Omega_{C O}^{k}
$$

defined by the convolution

$$
\left(a^{1}, a^{2}, \ldots, a^{m}\right) \mapsto a^{1} * a^{2} * \cdots * a^{m}
$$

is continuous.

Proof. (proof of Theorem 4.1.4) Following the general methods developed in [17, Chapter III. 1. $\alpha$, we deduce that the multilinear functional $\tau_{C}$ defines a cyclic cocycle on $C_{c}^{\infty}(H(\mathcal{F}, \Gamma))$, which is determined by $\left(\overline{\Omega^{*}}, \delta, \bar{\int}_{C}\right)$ as explained in [17, Proposition 4, 190.

For the continuity we argue as follows.

We shall first prove that the multilinear map

$$
C_{c}^{\infty}(G)_{L F} \times \cdots \times C_{c}^{\infty}(G)_{L F} \rightarrow C_{c}^{\infty}\left(G, \Lambda^{k}\left(t^{*} \nu^{*}\right)\right)_{C O}
$$

given by

$$
\left(a^{0}, a^{1}, \ldots, a^{k}\right) \mapsto\left(a^{0} * \delta a^{1} * \cdots * \delta a^{k}\right)_{11}
$$

is continuous.

By a straightforward computation, one easily gets that the transverse $k$-form $\left(a^{0} * \delta a^{1} * \cdots * \delta a^{k}\right)_{11}$ is a polynomial in the variables $a^{i}, d_{Q} a^{i}$ and $\theta$. Now, the transverse derivative $a \mapsto d_{Q} a$ clearly determines a continuous operator from $C_{c}^{\infty}(G)_{L F}$ to $C_{c}^{\infty}\left(G, t^{*}\left(\nu^{*}\right)\right)_{L F}$. Moreover, convolution by the fixed element $\theta$ is also continuous with respect to the LF-topology.

By applying Lemma 4.1.5 to all monomials appearing in $\left(a^{0} * \delta a^{1} * \cdots * \delta a^{k}\right)_{11}$ and summing them up in $C_{c}^{\infty}\left(G, \Lambda^{k}\left(t^{*} \nu^{*}\right)\right)_{C O}$, we get indeed that the multilinear map 4.19 is continuous with respect to the LF-topology on $C_{c}^{\infty}(G)$ and the CO-topology on $C_{c}^{\infty}\left(G, \Lambda^{k} t^{*}\left(\nu^{*}\right)\right)$.

Next, since we are assuming $M$ compact, the spaces $C^{\infty}\left(M, \Lambda^{*}\left(\nu^{*}\right)\right)_{L F}$ and $C^{\infty}\left(M, \Lambda^{*}\left(\nu^{*}\right)\right)_{C O}$ turn out to coincide and the resulting topology is a Fréchet topology. Moreover, we can choose a compact complete transversal $T$ to the foliation $\mathcal{F}$ and consider the space of differential forms $\Omega^{*}(T)$ with its natural LF-topology and the corresponding space of transverse differential forms 
$\Omega^{*}(\operatorname{Tr}(\mathcal{F}, \Gamma))$ (see Section 4.1.1) endowed with the quotient topology. Hence, the restriction of the map 4.19 to $M$ is continuous and the integration along the leaves, as defined in 4.3 , is also continuous.

Hence, the $k$-linear map

$$
\left(a^{0}, a^{1}, \ldots, a^{k}\right) \mapsto \int_{\mathcal{F}}\left(\left(a^{0} * \delta a^{1} * \cdots * \delta a^{k}\right)_{11}\right)_{\mid M}
$$

is continuous with respect to the LF-topology on $C_{c}^{\infty}(G)$ and the quotient topology on $\Omega^{k}(\operatorname{Tr}(\mathcal{F}, \Gamma))$. Finally, by definition an invariant $k$-current is a continuous linear form on $\Omega^{k}(\operatorname{Tr}(\mathcal{F}, \Gamma))$. Therefore,

$$
\left(a^{0}, a^{1}, \ldots, a^{k}\right) \mapsto\left\langle\int_{\mathcal{F}}\left(\left(a^{0} * \delta a^{1} * \cdots * \delta a^{k}\right)_{11}\right)_{\mid M}, C\right\rangle
$$

is continuous with respect to the LF-topology on $C_{c}^{\infty}(G)$. This concludes the proof.

From now on and unless otherwise specified, on $C_{c}^{\infty}(H(\mathcal{F}, \Gamma), E)$ we will always consider the LF-topology.

Remark 4.4. In [7] it is stated that the cyclic cocycle $\tau_{C}$ on $C_{c}^{\infty}(H(\mathcal{F}), E)$ is continuous with respect to the compact-open $C^{\infty}$ topology. Unfortunately, with respect to this topology the cocycle is only separately continuous.

Remark 4.5. The construction we have performed is formally the same as that done in [6] for the holonomy groupoid of the foliation. However, since we are working with the twisted holonomy groupoid we have produced different cyclic cocycles.

Let $(M, \mathcal{F}, \Gamma)$ be a codimension $q$ transversely oriented foliated $\Gamma$-manifold and assume that the action of $\Gamma$ on the normal bundle $\nu$ to the foliation consists of orientation-preserving bundle morphisms. We have shown in Example 4.1 that in this case there is always an invariant $q$-current $\int$ associated to the transverse orientation.

Definition 4.1. The transverse fundamental class of the transversely oriented foliated $\Gamma$-manifold $(M, \mathcal{F}, \Gamma)$, with $\Gamma$-action on the normal bundle $\nu$ given by orientation-preserving morphisms, is the class $[\tau] \in H C^{q}\left(C_{c}^{\infty}(H(\mathcal{F}, \Gamma), E)\right)$ of the cocycle associated to the invariant current $\int$ as described in Theorem 4.1.4

Remark 4.6. The restriction of any transverse fundamental cyclic cocycle $\tau$ to the smooth convolution algebra $C_{c}^{\infty}\left(H(\mathcal{F}), \Omega^{1 / 2} H(\mathcal{F})\right)$ of the holonomy groupoid coincides with the transverse fundamental cyclic cocycle as defined for instance in [17, Chapter III, Section 7, $\alpha$. More generally this holds for all cyclic cocycles coming from $P_{\Gamma}$-invariant currents.

Remark 4.7. All the constructions of the last two sections hold more generally for any foliation groupoid in the sense of [19]. 


\subsubsection{Equivariant cyclic cocycles}

For any foliated $\Gamma$-manifold $(M, \mathcal{F}, \Gamma)$, the cyclic cocycles on the smooth algebra $C_{c}^{\infty}(H(\mathcal{F}, \Gamma), E)$ introduced in the previous section give rise to twisted and equivariant cocycles. In this section, we will show that any cyclic cocycle $\tau_{C}$ associated to an invariant current determine in a natural way an equivariant cyclic cocycle, if the group $\Gamma$ is compact.

We keep denoting the twisted holonomy groupoid $H(\mathcal{F}, \Gamma)$ of $(M, \mathcal{F}, \Gamma)$ simply by $G$ and the bundle of half densities over it by $\Omega^{1 / 2}$ instead of $\Omega^{1 / 2} H(\mathcal{F}, \Gamma)$. Moreover, the bundle of leafwise densities on $M$ will be denoted by $\Omega_{\mathcal{F}}^{1 / 2}$.

As already mentioned in the introduction many ideas of this section are taken from [7, even if our treatment is more general, not assuming anything about the leafwise diffeomorphisms.

Thus, let $(M, \mathcal{F}, \Gamma)$ denote a foliated $\Gamma$-manifold and let $G$ be its twisted holonomy groupoid. Let $\Gamma$ to be a compact Lie group.

Let $\nu=T M / T(\mathcal{F})$ be the normal bundle to the foliation and let us choose a horizontal distribution $Q \subseteq T M$ that is invariant with respect to the action of $\Gamma$ on $T M$ given by differentials. Being $\Gamma$ compact, such horizontal distributions will always exist. In fact, the short exact sequence of $\Gamma$-equivariant vector bundles

$$
0 \rightarrow T(\mathcal{F}) \rightarrow T M \stackrel{\pi}{\rightarrow} \nu \rightarrow 0
$$

admits a linear splitting, i.e. a (not necessarily equivariant) bundle morphism $s^{\prime}: \nu \rightarrow T M$ over the identity of $M$, with $\pi \circ s^{\prime}=i d$. Now, since $\Gamma$ is compact, one can average $s^{\prime}$ with respect to the Haar measure of $\Gamma$ and obtain in this way an equivariant splitting $s$ such that $\pi \circ s=i d$.

Finally, let $E$ denote a $\Gamma$-equivariant bundle endowed with a $\Gamma$-invariant Hermitian metric and with a $\Gamma$-invariant connection $\nabla$.

By Theorem 1.5.7, for any $h \in \Gamma$ there exists a smooth global bisection

$$
\sigma_{h}: M \rightarrow G
$$

such that

- $t \circ \sigma_{h}=h$;

- for any $x \in M$, the holonomy along $\sigma_{h}(x)$ coincides with the germ $\operatorname{Hol}(f)_{x}$ at $x$.

Through $\sigma_{h}$ we have defined the operator (see 2.17)

$$
S(h)=\left\{S(h)_{x}\right\}_{x \in M}: L_{\tau}^{2}\left(G, t^{*}(E)\right) \rightarrow L_{\tau}^{2}\left(G, t^{*}(E)\right) .
$$

According to Proposition 2.1.14 we know that $S(h)$ defines a multiplier for the smooth convolution algebra $C_{c}^{\infty}(G, E)$, i.e. $S(h) \circ R(k), R(k) \circ S(h) \in$ $\left.C_{c}^{\infty} G, E\right)$. As usual, we understand under $R(k)$ the action of $k \in C_{c}^{\infty}(G, E)$ on $L_{\tau}^{2}\left(G, t^{*}(E)\right)$ through the right regular representation (see 2.8). Since this representation is faithful, we will identify $C_{c}^{\infty}(G, E)$ with its image using the 
same notation $k$ to denote an element of $C_{c}^{\infty}(G, E)$ and the corresponding operator $R(k)$, if there is no cause of confusion.

The compositions $S(h) \circ k$ and $k \circ S(h)$ defines linear actions of $\Gamma$ on $C_{c}^{\infty}(G, E)$, that are explicitly given by the formulas

$$
(S(h) \circ k)(\gamma)=k_{h}^{l}(\gamma)=\left(A_{h} \otimes i d\right) \cdot k\left(\sigma_{h^{-1}}(t(\gamma)) \gamma\right)
$$

and

$$
(k \circ S(h))(\gamma)=k_{h}^{r}(\gamma)=\left(i d \otimes A_{h^{-1}}^{*}\right) \cdot k\left(\sigma_{h^{-1}}(h t(\gamma)) h(\gamma)\right)
$$

for all $k \in C_{c}^{\infty}(G, E), h \in \Gamma$ and $\gamma \in \Gamma$. See Proposition 2.1.14 and Remark 2.2 for notation and the explicit computations.

These actions can be extended to $\Omega_{E}^{*}$. Indeed, the bundle $E N D(E) \otimes \Omega^{1 / 2} \otimes$ $t^{*}\left(\Lambda^{*} \nu^{*}\right)$ is $\Gamma$-equivariant, the action $\alpha_{h}$ on $t^{*}\left(\Lambda^{*} \nu^{*}\right)$ being induced canonically by the action of $\Gamma$ on $\nu$ and explicitly determined by the transpose $\left(h^{-1}\right)_{*}^{t}$ of the differential of $h^{-1}$ acting on the fibres of $\Lambda^{*} \nu^{*}$.

Remark 4.8. Notice that for all $\gamma \in G,\left(\alpha_{h}\right)_{\gamma}=\lambda_{\sigma_{h}(t(\gamma))}$, since the transverse action of $h$ is encoded in the bisection $\sigma_{h}$.

Remark 4.9. Using the $\Gamma$-invariant horizontal distribution $Q$, that we have chosen, we also have that the bundle $E N D(E) \otimes \Omega^{1 / 2} \otimes t^{*}\left(\Lambda^{*} Q^{*}\right)$ is $\Gamma$-equivariant. Moreover, the isomorphism between $E N D(E) \otimes \Omega^{1 / 2} \otimes t^{*}\left(\Lambda^{*} \nu^{*}\right)$ and $E N D(E) \otimes$ $\Omega^{1 / 2} \otimes t^{*}\left(\Lambda^{*} Q^{*}\right)$ is equivariant.

For any $\omega \in \Omega_{E}^{k}$ and $h \in \Gamma$ we set

$$
\begin{array}{r}
\omega_{h}^{l}(\gamma)=\left(A_{h} \otimes i d \otimes\left(h^{-1}\right)_{*}^{t}\right) \cdot \omega\left(\sigma_{h^{-1}}(t(\gamma)) \gamma\right) \\
\omega_{h}^{r}(\gamma)=\left(i d \otimes A_{h^{-1}}^{*} \otimes i d\right) \cdot \omega\left(\sigma_{h^{-1}}(h t(\gamma)) h(\gamma)\right)
\end{array}
$$

The notation expresses explicitly that we are acting separately on the target part and on the source part of the section $\omega$.

Remark 4.10. Viewing (see [26]) an element $\omega \in \Omega_{E}^{k}$ as the Schwartz kernel of a $G$-invariant family of smoothing operators $P^{\omega}=\left(P_{x}^{\omega}\right)_{x \in M}$ such that

$$
P_{x}^{\omega}: C_{c}^{\infty}\left(G, t^{*}(E)\right) \rightarrow C_{c}^{\infty}\left(G, t^{*}\left(E \otimes \Lambda^{k} Q^{*}\right),\right.
$$

it can be shown arguing as in Proposition 2.1.14 that the composition of operators $S(h) \circ P^{\omega}$ is an operator with Schwartz kernel given by $\omega_{h}^{l}$. Similarly the composition $P^{\omega} \circ S(h)$ is an operator with Schwartz kernel $\omega_{h}^{r}$.

Proposition 4.1.6. Let $(M, \mathcal{F}, \Gamma)$ be a foliated $\Gamma$-manifold and let $Q \subseteq T M$ be a $\Gamma$-invariant horizontal distribution.

1. For any $\omega \in \Omega_{E}^{k}, \eta \in \Omega_{E}^{l}$, it holds that

$$
\omega * \eta_{h}^{l}=\omega_{h}^{r} * \eta
$$


2. If the bundle $E$ is endowed with a $\Gamma$-invariant connection and if $\nabla_{Q}$ denotes the corresponding transverse differentiation on $\Omega_{E}^{*}$, then for any $k \in \Omega_{E}^{0}$ and $h \in \Gamma$

$$
\nabla_{Q}(S(h) \circ k)=\nabla_{Q}\left(k_{h}^{l}\right)=\left(\nabla_{Q} k\right)_{h}^{l}
$$

Proof. 1). For notational simplicity, we assume that the bundle $E$ is trivial of rank one. For general bundles the result follows exactly from the same argument. Moreover, we will not denote explicitly the action of $\Gamma$ on densities, being obvious where it is used.

Recall that by definition and for any $\gamma \in G, \gamma: x \rightarrow y$

$$
\begin{aligned}
& \left(\omega * \eta_{h}^{l}\right)(\gamma)=\int_{G^{y}} \omega\left(\gamma_{1}\right) \wedge \lambda_{\gamma_{1}}\left(\eta_{h}^{l}\left(\gamma_{1}^{-1} \gamma\right)\right)= \\
& =\int_{G^{y}} \omega\left(\gamma_{1}\right) \wedge \lambda_{\gamma_{1}}\left(\alpha_{h} \eta\left(\sigma_{h^{-1}}\left(s\left(\gamma_{1}\right)\right) \gamma_{1}^{-1} \gamma\right)\right) .
\end{aligned}
$$

Now, setting $\gamma^{\prime}=\gamma_{1} \sigma_{h}\left(h^{-1} s\left(\gamma_{1}\right)\right)$ one gets

$$
\omega\left(\gamma_{1}\right)=\omega\left(\gamma^{\prime} \sigma_{h}\left(h^{-1} s\left(\gamma_{1}\right)\right)^{-1}\right)=\omega\left(\gamma^{\prime} \sigma_{h}^{-1}\left(h s\left(\gamma^{\prime}\right)\right)\right)
$$

Moreover

$$
\left(\gamma^{\prime}\right)^{-1}=\sigma_{h}\left(h^{-1} s\left(\gamma_{1}\right)\right)^{-1} \gamma_{1}^{-1}=\sigma_{h^{-1}}\left(s\left(\gamma_{1}\right)\right) \gamma_{1}^{-1} .
$$

Hence from this computation, the fact that $\left(\alpha_{h}\right)_{h^{-1} s\left(\gamma_{1}\right)}=\lambda_{\sigma_{h}\left(h^{-1} s\left(\gamma_{1}\right)\right)}$ and the invariance of the integral of densities with respect to diffeomorphisms, we finally deduce that

$$
\begin{gathered}
\int_{G^{y}} \omega\left(\gamma_{1}\right) \wedge \lambda_{\gamma_{1}}\left(\alpha_{h} \eta\left(\sigma_{h^{-1}}\left(s\left(\gamma_{1}\right)\right) \gamma_{1}^{-1} \gamma\right)\right)= \\
\left.=\int_{G^{y}} \omega\left(\gamma^{\prime} \sigma_{h}^{-1}\left(h s\left(\gamma^{\prime}\right)\right)\right) \wedge \lambda_{\gamma_{1}} \lambda_{\sigma_{h}\left(h^{-1} s\left(\gamma_{1}\right)\right)} \eta\left(\left(\gamma^{\prime}\right)^{-1} \gamma\right)\right)= \\
=\int_{G^{y}} \omega_{h}^{r} \wedge \lambda_{\gamma^{\prime}} \eta\left(\left(\gamma^{\prime}\right)^{-1} \gamma\right)=\left(\omega_{h}^{r} * \eta\right)(\gamma) .
\end{gathered}
$$

2). Let us first assume that the bundle $E$ is trivial of rank 1 and let us denote the transverse derivation simply by $d_{Q}$.

Given an arbitrary element $k \in C_{c}^{\infty}\left(G, \Omega^{1 / 2}\right)$, we can write it as

$$
k=f s^{*}(\alpha) t^{*}(\alpha),
$$

where $f \in C_{c}^{\infty}(G), \alpha \in C^{\infty}\left(M, \Omega_{\mathcal{F}}^{1 / 2}\right)$. With this notation, it is easy to check that for any $h \in \Gamma$

$$
k_{h}^{l}=\left(f s^{*}(\alpha) t^{*}(\alpha)\right)_{h}^{l}=f_{h}^{l} s^{*}(\alpha) t^{*}(h \cdot \alpha),
$$

where

$$
f_{h}^{l}(\gamma)=f\left(\sigma_{h^{-1}}(t(\gamma)) \gamma\right)
$$

and

$$
(h \cdot \alpha)(t(\gamma))=h \cdot \alpha\left(h^{-1}(t(\gamma))\right)
$$

is the usual action on $h$ on half densities. On the density $s^{*}(\alpha)$ there is no action of $h$ since we only act on the target part of $k$.

Thus, the claim follows if we establish the two following equalities 
1. $d_{Q}\left(f_{h}^{l}\right)=\left(d_{Q} f\right)_{h}^{l}$

2. $t^{*}\left(d_{Q}(h \cdot \alpha)\right)=t^{*}\left(h \cdot d_{Q}(\alpha)\right)$

To show the first one, we proceed exactly as in [7, Lemma 4.16. We sketch the argument for the sake of completeness.

Let $\gamma \in G, \gamma: x \rightarrow y$ and consider the element $\gamma_{1}=\sigma_{h^{-1}}(y) \gamma$. Further, take any $X \in t^{*}(Q)_{\gamma} \cong Q_{y}$.

The normal vector $X$ gives rise to a tangent vector $\hat{X} \in T_{\gamma} G$ determined by the following requirements,

$$
s_{*}(\hat{X})=\lambda_{\gamma^{-1}} X \quad t_{*}(\hat{X})=X .
$$

Since the anchor map of the groupoid $(s, t)$ is an immersion we get that $\hat{X}$ is unique. The vector $X$ defines also a vector $Y \in T_{h^{-1}(y)} M$ by putting $Y=$ $h_{*}^{-1}(X)=\lambda_{\sigma_{h^{-1}}(y)}(X)$ and then the corresponding tangent vector $\hat{Y} \in T_{\gamma_{1}} G$ determined by setting

$$
s_{*}(\hat{Y})=\lambda_{\gamma_{1}^{-1}}(Y)=\lambda_{\left(\sigma_{h}^{-1}(y) \gamma\right)^{-1}}(Y)=\lambda_{\gamma^{-1}} X \quad t_{*}(\hat{Y})=Y .
$$

Now, by definition

$$
d_{Q}\left(f_{h}^{l}\right)(X)=d\left(f_{h}^{l}\right)(\hat{X})
$$

and

$$
d_{Q}(f)(Y)=d f(\hat{Y}) .
$$

Now, consider any smooth local curve $\gamma_{t} \subseteq G$ passing through $\gamma$ for $t=0$ and defining the tangent vector $\hat{X}$. Then, it can be shown that the curve $\beta_{t}=$ $\sigma_{h^{-1}}\left(t\left(\gamma_{t}\right)\right) \gamma_{t} \subseteq G$ passes through $\gamma_{1}$ and defines the vector $\hat{Y}$. Since $f_{h}^{l}\left(\gamma_{t}\right)=$ $f\left(\beta_{t}\right)$, we get

$$
\begin{gathered}
d_{Q}\left(f_{h}^{l}\right)(X)=d\left(f_{h}^{l}\right)(\hat{X})=\frac{d}{d t}\left(f_{h}^{l}\left(\gamma_{t}\right)\right)_{\mid t=0}= \\
=\frac{d}{d t}\left(f\left(\beta_{t}\right)\right)_{\mid t=0}=d f(\hat{Y})=d_{Q}(f)(Y)=d_{Q} f\left(h_{*}^{-1}(X)\right)= \\
=\left(\left(h^{-1}\right)_{*}^{t} d_{Q} f\right)(X) .
\end{gathered}
$$

Thus, we have proved the first equality.

For the second equality we argue as follows. Let us fix a $\Gamma$-invariant positive one-density $\lambda \in C^{\infty}\left(M, \Omega_{\mathcal{F}}^{1}\right)$. Then every half-density $\alpha \in C^{\infty}\left(M, \Omega_{\mathcal{F}}^{1 / 2}\right)$ can be written as $\alpha=f|\lambda|^{1 / 2}$ and the transverse derivation of $\alpha$ is given by the formula

$$
d_{Q}(\alpha)=d_{Q}\left(f|\lambda|^{1 / 2}\right)=d_{Q}(f)|\lambda|^{1 / 2}+\frac{1}{2} f|\lambda|^{1 / 2} k(\lambda)
$$

where $k(\lambda) \in C^{\infty}\left(M, Q^{*}\right)$ is the transverse derivation of $\lambda$ as defined in the previous section (see also [35, pag. 50).

Now, from the invariance of $\lambda$, one has that

$$
\begin{gathered}
d_{Q}(h \cdot \alpha)=d_{Q}\left((h \cdot f)|\lambda|^{1 / 2}\right)= \\
=d_{Q}(h \cdot f)|\lambda|^{1 / 2}+(h \cdot f)|\lambda|^{1 / 2} k(\lambda)=h \cdot d_{Q}(f)|\lambda|^{1 / 2}+(h \cdot f)|\lambda|^{1 / 2} k(\lambda) .
\end{gathered}
$$


On the other side

$$
h \cdot\left(d_{Q}(\alpha)\right)=h \cdot d_{Q}(f)|\lambda|^{1 / 2}+(h \cdot f)(h \cdot k(\lambda))|\lambda|^{1 / 2} .
$$

Hence, the equality follows if we show that $h \cdot k(\lambda)=k(\lambda)$, for any $h \in \Gamma$. But applying the definition of $k(\lambda)$ and using the invariance of $\lambda$, it follows straightforwardly that $k(\lambda)$ is indeed $\Gamma$-invariant.

This proves the secong equality and thus the claim in the case $E$ is trivial of a rank 1 .

For general $E$, observe that any element $k \in C_{c}^{\infty}(G, E)$ can be written as

$$
k=f s^{*}(\alpha) t^{*}(\beta),
$$

where $f \in C_{c}^{\infty}(G), \alpha \in C^{\infty}\left(M, E^{*} \otimes \Omega_{\mathcal{F}}^{1 / 2}\right)$ and $\beta \in C^{\infty}\left(M, E \otimes \Omega_{\mathcal{F}}^{1 / 2}\right)$. Hence, provided $E$ is endowed with a $\Gamma$-invariant connection $\nabla$, we can apply the same argument as above using $E$-valued densities.

Recall (cf. Section 4.1.1) that the pseudogroup associated to the reduced étale groupoid $G_{T}^{T}=H(\mathcal{F}, \Gamma)_{T}^{T}$ with respect to a complete transversal $T$ is denoted by $P_{\Gamma}$.

Proposition 4.1.7. Let $C \in C_{m}(\operatorname{Tr}(\mathcal{F}, \Gamma))$ be a closed $P_{\Gamma}$-invariant current, with $m>0$, and let $\tau_{C}$ be the corresponding cyclic cocycle on $C_{c}^{\infty}(G, E)$ as given by Theorem 4.1.4. Then, one has that

$$
\tau_{C}\left(k^{0}, \ldots, k^{i} \circ S(h), k^{i+1}, \ldots, k^{m}\right)=\tau_{C}\left(k^{0}, \ldots, k^{i}, S(h) \circ k^{i+1}, \ldots, k^{m}\right)
$$

for every $k^{i} \in C_{c}^{\infty}(G, E), h \in \Gamma$.

If $C \in C_{0}(\operatorname{Tr}(\mathcal{F}, \Gamma))$ is a closed invariant 0-current, then it holds

$$
\tau_{C}(k \circ S(h))=\tau_{C}(S(h) \circ k)
$$

for $k \in C_{c}^{\infty}(G, E), h \in \Gamma$.

Proof. We argue as in [7. Let us first prove the proposition for $m>0$. Using the fact that $\tau_{C}$ is cyclic, it suffices to show that

$$
\tau_{C}\left(k^{0} \circ S(h), \ldots, k^{m}\right)=\tau_{C}\left(k^{0}, S(h) \circ k^{1}, \ldots, k^{m}\right) .
$$

Now, recall that the $\tau_{C}$ is defined by the formula

$$
\tau_{C}\left(k^{0}, k^{1}, \ldots, k^{m}\right)=\int_{C} \operatorname{tr}\left(\left(\left(k^{0} * \delta k^{1} * \cdots * \delta k^{m}\right)_{11}\right)_{\mid M}\right.
$$

where $*$ is the product of the algebra $\overline{\Omega_{E}^{*}}=M_{2}\left(\Omega_{E}^{*}\right)$ and $\delta$ the derivation on it defined in the previous section. Thus, we only need to study the $(1,1)$ component in $M_{2}\left(\Omega_{E}^{*}\right)$ of the above product.

Computing explicitly $k^{0} * \delta k^{1} * \cdots * \delta k^{m}$ in terms of the product in $\Omega_{E}^{*}$, one gets two types of terms involving the action of the multiplier $S(h)$. First one obtains terms of the form $k^{0}\left(S(h) \circ k^{1}\right) Q$, where $Q$ is a polynomial in the variables 
$k^{i}, \nabla_{Q} k^{i}$ and the curvature term $\theta$, for which the result is immediate. The second type of terms are given by $k^{0} \nabla_{Q}\left(S(h) \circ k^{1}\right) Q$. Applying now Proposition 4.1.6. we deduce that $k^{0} \nabla_{Q}\left(S(h) \circ k^{1}\right)=\left(k^{0} \circ S(h)\right) \nabla_{Q} k^{1}$, which implies the result.

If now $C \in C_{0}(\operatorname{Tr}(\mathcal{F}, \Gamma))$, we argue as follows.

For any other group element $g \in \Gamma$ we will prove the formula

$$
\tau_{C}(g(k \circ S(h)))=\tau_{C}(k \circ S(h)),
$$

from which Equation 4.27 easily follows.

Since by definition of $\int_{C}$ we restrict to the $M$, one can assume the support of $\tilde{k}=k \circ S(h)$ to be contained in a foliated chart $U$ of $M$. Then, the support of $g(\tilde{k})$ is contained in the foliated chart $V=g(U)$.

By the invariance of the integral of 1 -densities with respect to diffeomorphisms we know that

$$
\int_{\mathcal{F}} g(\tilde{k})=\int_{V / \mathcal{F}} g(\tilde{k})=\int_{U / \mathcal{F}} \tilde{k} .
$$

This implies that

$$
\left\langle\int_{V / \mathcal{F}} g(\tilde{k}), C\right\rangle=\left\langle\int_{U / \mathcal{F}} \tilde{k},\left(g^{-1}\right)_{*} C\right\rangle
$$

Hence, if $\left(g^{-1}\right)_{*} C=C$, or equivalently $g_{*} C=C$, the result follows. Let $S \subseteq U$ a transversal and set $T=g(S) \subseteq V$. Since $g$ is lifted by the bisection $\sigma_{g} \in$ $\operatorname{Bis}(H(\mathcal{F}, \Gamma))$, one can cover $S$ with open sets $\Omega_{j}$ in such a way that $g_{\mid \Omega_{j}}=$ $\lambda_{\sigma_{g}\left(y_{j}\right)}$, for suitable $y_{j} \in \Omega_{j}$. Since $C$ is $P_{\Gamma}$-invariant, we have

$$
g_{*}(C)_{\mid g\left(\Omega_{j}\right)}=\lambda_{\sigma_{g}\left(y_{j}\right)}^{*}(C)_{\mid g\left(\Omega_{j}\right)}=C_{\mid g\left(\Omega_{j}\right)} .
$$

This completes the proof.

As consequence of the propositions above, we get the following theorem. Recall that $C_{c}^{\infty}(H(\mathcal{F}, \Gamma), E)$ carries its natural LF-topology.

Theorem 4.1.8. Let $(M, \mathcal{F}, \Gamma)$ a foliated $\Gamma$-manifold, with $\Gamma$ compact, and let $Q \subseteq T M$ a $\Gamma$-invariant horizontal distribution.

Let $C \in C_{m}(\operatorname{Tr}(\mathcal{F}, \Gamma))$ closed and let $\tau_{C}$ be the corresponding cyclic cocycle on $C_{c}^{\infty}(H(\mathcal{F}, \Gamma), E)$. Then the formula

$$
\bar{\varphi}_{C}\left(k^{0}, k^{1}, \ldots, k^{m} \mid h\right)=\tau_{C}\left(S(h) \circ k^{0}, k^{1}, \ldots, k^{m}\right)
$$

defines an equivariant cyclic $m$-cocycle on $\left(C_{c}^{\infty}(H(\mathcal{F}, \Gamma), E), \Gamma\right)$.

Proof. Continuity follows immediately from the continuity of $\tau_{C}$ and of the linear action defined by the multipliers $S(h)$. Moreover, the fact that it is a closed cyclic invariant cochain is an immediate corollary of Proposition 4.1.7 and Lemma 3.3 .2 . 
Remark 4.11. Notice that evaluating the equivariant cyclic $\bar{\varphi}_{C}$ at the identity $e \in \Gamma$, we get the (non-equivariant) cyclic cocycle $\tau_{C}$, which we started with. Heuristically, we can think of $\bar{\varphi}_{C}$ as being a delocalization of $\tau_{C}$ outside the identity element of $\Gamma$.

Remark 4.12. As suggested by Ralf Meyer, using the invariance of equivariant cyclic cohomology under equivariant Morita equivalences one can conceive an alternative method to get equivariant extension of nonequivariant cyclic cocycles.

More precisely, Remark 1.15 in Chapter 1 implies that $C_{c}^{\infty}(H(\mathcal{F}, \Gamma), E)$ with its canonical $\Gamma$-action is equivariantly Morita equivalent to $C_{c}^{\infty}(H(\mathcal{F}, \Gamma), E)$ with trivial $\Gamma$-action. Thus, the equivariant cyclic cohomology of $C_{c}^{\infty}(H(\mathcal{F}, \Gamma), E)$ is isomorphic to that of $C_{c}^{\infty}(H(\mathcal{F}, \Gamma), E)$ with trivial action, that is in turn isomorphic to the $C(\Gamma)$-valued nonequivariant cyclic cohomology of $C_{c}^{\infty}(H(\mathcal{F}, \Gamma), E)$. Hence, once one has a nonequivariant cyclic cocycle $\tau$ on $C_{c}^{\infty}(H(\mathcal{F}, \Gamma), E)$ one obtains an equivariant cyclic cocycle on the algebra $C_{c}^{\infty}(H(\mathcal{F}, \Gamma), E)$ with trivial $\Gamma$-action by extending $\tau$ constantly for any element of $\Gamma$. Then, using the isomorphisms mentioned above, one gets an equivariant cyclic cocycle $\bar{\varphi}$ on $C_{c}^{\infty}(H(\mathcal{F}, \Gamma), E)$ endowed with the canonical $\Gamma$-action, whose evaluation at the identity is $\tau$.

We believe that the methods shown in Theorem 4.1.8 and mentioned here yield eventually the same equivariant cyclic cocycles but we did not work out the details.

An important example of the above theorem is provided by the transverse fundamental cyclic cocycles of a foliated $\Gamma$-manifold as defined in Definition 4.1

Corollary 4.1.9. Let $(M, \mathcal{F}, \Gamma)$ be a foliated $\Gamma$-manifold of codimension $q$ with $\Gamma$ compact and let $Q$ denote a $\Gamma$-invariant horizontal distribution.

If $(M, \mathcal{F}, \Gamma)$ is transversely oriented and the action of $\Gamma$ on $Q$ is given by orientation-preserving bundle morphisms, then there exists an equivariant cyclic cocycle $\bar{\varphi}$ on $\left(C_{c}^{\infty}(H(\mathcal{F}, \Gamma), E), \Gamma\right)$ whose evaluation at $e \in \Gamma$ is the transverse fundamental cyclic $q$-cocycle $\tau$ on $\left(C_{c}^{\infty}(H(\mathcal{F}, \Gamma), E)\right)$ associated to the horizontal distribution $Q$.

The equivariant cyclic cocycle $\bar{\varphi}$ will be called an equivariant transverse fundamental cocycle of $(M, \mathcal{F}, \Gamma)$.

Using the $\Gamma$-equivariant inclusion $j: C_{c}^{\infty}(H(\mathcal{F}), E) \hookrightarrow C_{c}^{\infty}(H(\mathcal{F}, \Gamma), E)$, we can define an equivariant cyclic cocycle $\varphi_{C}$ on $\left(C_{c}^{\infty}(H(\mathcal{F}), E), \Gamma\right)$ by restriction. More precisely for every closed $C \in C_{m}(\operatorname{Tr}(\mathcal{F}, \Gamma))$ we can set

$$
\varphi_{C}\left(a^{0}, \ldots, a^{m} \mid h\right)=j^{*}\left(\bar{\varphi}_{C}\right)\left(a^{0}, \ldots, a^{m} \mid h\right)
$$

for all $a^{i} \in C_{c}^{\infty}(H(\mathcal{F}), E), h \in \Gamma$, defining an equivariant cyclic cocycle on $C_{c}^{\infty}(H(\mathcal{F}), E)$. We will say that $\varphi_{C}$ is the restriction of $\bar{\varphi}_{C}$ on the holonomy groupoid $H(\mathcal{F})$. 
We are now able to define higher Lefschetz numbers.

Let $P \in \Psi_{c}^{m}(H(\mathcal{F}, \Gamma), E)$ be a $\Gamma$-invariant elliptic pseudodifferential $H(\mathcal{F}, \Gamma)$-operator and let $h \in \Gamma$. Recall that the smooth Lefschetz class $L_{\infty}([h], P)$ on $H(\mathcal{F}, \Gamma)$ of the conjugacy class $[h]$ relative to $P$ is defined as

$$
L_{\infty}([h], P)=\frac{i n d_{C^{\infty}}^{\Gamma}(P)}{1_{R[\Gamma]}} \in K_{0}^{\Gamma}\left(C_{c}^{\infty}(H(\mathcal{F}, \Gamma), E)\right)_{[h]}
$$

where $K_{0}^{\Gamma}\left(C_{c}^{\infty}(H(\mathcal{F}, \Gamma), E)\right)_{[h]}$ is the module localized at the prime ideal $I_{[h]}$ of $R[\Gamma]$ determined by $[h]$ (cf. [2.2.3).

Let $C$ be a closed even dimensional invariant current and let $\bar{\varphi}_{C}$ be the corresponding equivariant cyclic cocycle on $\left(C_{c}^{\infty}(H(\mathcal{F}, \Gamma), E), \Gamma\right)$ as given by Theorem 4.1.8 By Proposition 3.4.5, the equivariant cocycle $\bar{\varphi}_{C}$ defines an additive map

$$
\left\langle\cdot, \bar{\varphi}_{C}\right\rangle_{[h]}: K_{0}^{\Gamma}\left(C_{c}^{\infty}(H(\mathcal{F}, \Gamma), E)\right)_{[h]} \rightarrow \mathbb{C} .
$$

Definition 4.2. The $\bar{\varphi}_{C}$-Lefschetz number on $H(\mathcal{F}, \Gamma)$ of $[h]$ with respect to the $\Gamma$-invariant elliptic operator $P$ is the number

$$
L_{\bar{\varphi}_{C}}([h], P)=\left\langle L_{\infty}([h], P), \bar{\varphi}_{C}\right\rangle_{I_{[h]}}
$$

Using again Proposition 3.4 .5 and the very definition of $L_{\infty}([h], P)$, one gets that

$$
L_{\bar{\varphi}_{C}}([h], P)=\chi_{\bar{\varphi}_{C}}([h])\left(\operatorname{ind}_{C^{\infty}}^{\Gamma}(P)\right)=\chi_{\bar{\varphi}_{C}}(h)\left(\operatorname{ind}_{C^{\infty}}^{\Gamma}(P)\right),
$$

i.e. the $\bar{\varphi}_{C}$-Lefschetz numbers depend directly on the equivariant index class without localizing (here we have used the notation of Section 2.2.3).

Now, restricting the cocycle $\bar{\varphi}_{C}$ to the holonomy groupoid $H(\mathcal{F})$ and denoting the resulting equivariant cocycle by $\varphi_{C}$, we obtain an additive map

$$
\left\langle\cdot, \varphi_{C}\right\rangle_{I_{[h]}}: K_{0}^{\Gamma}\left(C_{c}^{\infty}(H(\mathcal{F}), E)\right)_{[h]} \rightarrow \mathbb{C} .
$$

If $h \in \Gamma$ and $D \in \Psi_{c}^{\infty}(H(\mathcal{F}) ; E)$ is a $\Gamma$-invariant elliptic operator on the holonomy groupoid, we may take its Lefschetz class $\mathcal{L}_{\infty}([h], D) \in K_{0}^{\Gamma}\left(C_{c}^{\infty}(H(\mathcal{F}), E)\right)_{[h]}$ and set

Definition 4.3. The $\varphi_{C}$-Lefschetz number on $H(\mathcal{F})$ of $[h]$ with respect to the $\Gamma$-invariant elliptic operator $D$ is the scalar

$$
\mathcal{L}_{\varphi_{C}}([h], D)=\left\langle\mathcal{L}_{\infty}([h], D), \varphi_{C}\right\rangle_{I_{[h]}} .
$$

As above, one can deduce from Proposition 3.4.5 that the $\varphi_{C}-$ Lefschetz number on $H(\mathcal{F})$ only depends on the equivariant index class.

From Proposition 3.4 .4 and the discussion after it follows that these higher Lefschetz numbers do not depend on the group $\Gamma$ as far as this latter exists.

The Lefschetz numbers are important invariants associated to the diffeomorphism $h$ and the operators $P$ and $D$. We will see few properties of these invariants in the last section of the chapter. For othere results we refer to [7, [5] and [28. 


\subsubsection{Twisted cyclic cocycles}

If the group $\Gamma=\mathbb{Z}$, generated by a leaf-preserving diffeomorphism $h$, the procedure developed above allows to construct cyclic cocycles, which are equivariant with respect to $\mathbb{Z}$. However, since the group is no longer compact we will not use directly the equivariant cyclic theory, but rather the twisted theory (cf. Chapter 3). This will allow us to get Lefschetz-type invariants in situations that are slightly more general than those considered above.

Given a foliated manifold $(M, \mathcal{F})$ and $h \in \operatorname{Diff}(\mathcal{F})$ a leaf-preserving diffeomorphism, one can consider the foliated $\Gamma$-manifold $(M, \mathcal{F}, \Gamma)$, where $\Gamma$ is the cyclic group generated by $h$. Notice that $\Gamma$ could be isomorphic to $\mathbb{Z}$ or to $\mathbb{Z}_{n}$, where $h^{n}=i d$. Since we have already studied the case of a compact group, we will focus here on the case where $\Gamma=\mathbb{Z}$.

Then, let $(M, \mathcal{F}, \mathbb{Z})$ be a foliated $\mathbb{Z}$-manifold with $\mathbb{Z}$ generated by $h$ and consider its twisted holonomy groupoid $H(\mathcal{F}, \mathbb{Z})$. We assume that there exists a one-density $\lambda \in \Omega_{\mathcal{F}}^{1 / 2}$ which is preserved $h$ and that there exists a horizontal distribution $Q \subseteq T M$ complementary to $T(\mathcal{F})$ which is invariant under the action of $h$ given by differential. Moreover, let $E$ denote a $\mathbb{Z}$-equivariant Hermitian vector bundle endowed with a $\mathbb{Z}$-invariant connection.

Therefore, setting $\Gamma=\mathbb{Z}$ we can follow the same steps done in the previous section until Proposition 4.1.7.

More precisely, it follows that for any closed $P_{\mathbb{Z}}$-invariant current $C \in C_{m}(\operatorname{Tr}(\mathcal{F}, \mathbb{Z}))$ the corresponding cyclic cocycle $\tau_{C}$ on $C_{c}^{\infty}(H(\mathcal{F}, \mathbb{Z}), E)$ satisfies the fundamental relation

$$
\tau_{C}\left(k^{0}, \ldots, k^{i} \circ S(l), k^{i+1}, \ldots, k^{m}\right)=\tau_{C}\left(k^{0}, \ldots, k^{i}, S(l) \circ k^{i+1}, \ldots, k^{m}\right),
$$

for every $k^{i} \in C_{c}^{\infty}(H(\mathcal{F}, \mathbb{Z}), E), l \in \mathbb{Z}$ (if $m=0$, see 4.1.7).

Unfortunately, since $\mathbb{Z}$ is not compact we do not obtain an equivariant cyclic cocycle. However, arguing as in Theorem 4.1 .8 one immediately gets

Proposition 4.1.10. Let $(M, \mathcal{F}, \mathbb{Z})$ be a foliated $\mathbb{Z}$-manifold, with $\mathbb{Z}$ generated by the diffeomorphism $h \in \operatorname{Diff}(\mathcal{F})$. Let $\tau_{C}$ be the cyclic cocycle on $C_{c}^{\infty}(H(\mathcal{F}, \mathbb{Z}), E)$ associated to a closed $P_{\mathbb{Z}}$-invariant current $C \in C_{m}(\operatorname{Tr}(\mathcal{F}, \mathbb{Z}))$. The formula

$$
\bar{\varphi}_{C}^{h}\left(k^{0}, k^{1}, \ldots, k^{m}\right)=\tau_{C}\left(S\left(h^{-1}\right) \circ k^{0}, k^{1}, \ldots, k^{m}\right)
$$

defines a $h$-twisted cyclic $m$-cocycle on the pair $\left(C_{c}^{\infty}(H(\mathcal{F}, \mathbb{Z}), E), h\right)$.

Exactly as in the case where $\Gamma$ is compact, we have the important example represented by the transverse fundamental class. Precisely

Corollary 4.1.11. Let $(M, \mathcal{F}, \mathbb{Z})$ be a transversely oriented codimension $q$ foliated $\mathbb{Z}$-manifold and let $Q$ a $\mathbb{Z}$-invariant horizontal distribution. Assume that $h$ acts on $Q$ as an orientation-preserving bundle morphism.

Then the corresponding transverse fundamental cyclic cocycle $\tau$ of the algebra $\left(C_{c}^{\infty}(H(\mathcal{F}, \mathbb{Z}), E)\right)$ is the evaluation at $e \in \Gamma$ of a twisted cyclic cocycle $\bar{\varphi}_{C}^{h}$ of the pair $\left(C_{c}^{\infty}(H(\mathcal{F}, \mathbb{Z}), E), h\right)$. 
It is interesting to define twisted cyclic cocycles as above, in order to be able to treat the following situation. Let us assume that there exists a compact Lie group $W$ such that

- $\Gamma$ is a dense subgroup of $W$;

- $W$ acts only as foliation-preserving diffeomorphisms extending the action of $\Gamma$, i.e. there is an action $\alpha: W \rightarrow \operatorname{Diff}(M, \mathcal{F})$ extending that of $\Gamma$.

In this case, we have a foliated $\Gamma$-manifold, but not a foliated $W$-manifold, since by definition the group needs to act by leaf-preserving diffeomorphisms. However we can average over the group $W$ with respect to its Haar measure, in order to get the existence for instance of $W$-invariant one-densities on $M$ and invariant horizontal distribution complementary to the foliation.

Hence, taking $h \in \Gamma$ and considering $(M, \mathcal{F}, \mathbb{Z})$, with $\mathbb{Z}$ generated by $h$, all the required assumptions in Proposition 4.1.10 are satisfied and we get $h$-twisted cyclic cocycles $\bar{\varphi}_{C}^{h}$.

Now, we may restrict $\bar{\varphi}_{C}^{h}$ to the holonomy groupoid $H(\mathcal{F})$ getting the twisted cyclic cocycle $\varphi_{C}^{h}$. Applying Theorem 3.5.4, we get a map

$$
\left\langle\cdot, \varphi_{C}^{h}\right\rangle_{h}: K_{0}^{W}\left(C_{c}^{\infty}(H(\mathcal{F}), E)\right) \rightarrow \mathbb{C}
$$

that descends to the module of fractions $K_{0}^{W}\left(C_{c}^{\infty}(H(\mathcal{F}), E)\right)_{I_{[h]}}$. Hence, we set in this case the following definition

Definition 4.4. The $\varphi_{C}^{h}$-Lefschetz number of $[h]$ with respect to a $W$-invariant elliptic operator $D$ is the number

$$
\mathcal{L}_{\varphi_{C}^{h}}([h], D)=\left\langle\mathcal{L}_{\infty}([h], D), \varphi_{C}^{h}\right\rangle_{I_{[h]}}
$$

Thus we could define higher Lefschetz numbers as in the equivariant case, using the pairing between equivariant $K$-theory of the convolution algebra of the holonomy groupoid and its twisted cyclic cohomology.

\subsection{The equivariant Godbillon-Vey cyclic class}

This section is devoted to studying the Godbillon-Vey class for a transversely oriented codimension 1 foliated manifold. More comprehensive introductions are at the beginning of each subsection.

\subsubsection{The Godbillon-Vey class: classical definition}

We are going to introduce the Godbillon-Vey class for any transversely oriented foliated manifold $(V, \mathcal{F})$ of codimension 1 and present some of its basic features. Let $(M, \mathcal{F})$ be a transversely oriented foliated manifold of codimension 1 . Let $F=T(\mathcal{F}) \subseteq T M$ denote the tangent bundle to the foliation and let $\nu=T M / F$ be the normal bundle to the foliation. Recall that a foliation is transversely oriented if its normal bundle is oriented. 
In this case and since the foliation has codimension 1, there exists a 1-form $\omega \in \Omega^{1}(M)$ which defines the foliation, i.e. it is nonsingular and

$$
\operatorname{Ker}(\omega):=\left\{w \in T_{x} M: \omega_{x}(w)=0\right\}=F
$$

Let us consider the annihilator $I^{*}(F)$ defined by integrability of the vector bundle $F$, see 1.1.2. Since $I^{*}(F)$ is a differential graded ideal of $\Omega^{*}(M)$ and clearly $\omega \in I^{1}(F)$, it follows $d \omega \in I^{2}(F)$. Now, it is not very difficult to see that, in our case, $I^{*}(F)$ is actually a principal differential graded ideal of $\Omega^{*}(M)$ generated by $\omega$. Therefore it exists $\eta \in \Omega^{1}(M)$ so that

$$
d \omega=\eta \wedge \omega
$$

Moreover

$$
0=d^{2} \omega=d(\eta \wedge \omega)=d \eta \wedge \omega
$$

and, since $\omega$ is nonsingular, there is $\alpha \in \Omega^{1}(M)$

$$
d \eta=\alpha \wedge \omega .
$$

From these computations it follows easily that the differential form $\eta \wedge d \eta$ is a closed 3-form of $M$. Indeed

$$
d(\eta \wedge d \eta)=d \eta \wedge d \eta=\alpha \wedge \omega \wedge \alpha \wedge \omega=0
$$

Definition 4.5. The 3-dimensional cohomology class

$$
G V(\mathcal{F})=[\eta \wedge d \eta] \in H^{3}(M ; \mathbb{R})
$$

is called the Godbillon-Vey class of the transversely oriented codimension 1 foliation $(M, \mathcal{F})$.

The choice of $\omega$ and $\eta$, in the construction of $G V(\mathcal{F})$, is of course not unique; thus it might happen that the Godbillon-Vey class does depend also on the choice of these differential forms. However it does not as the following lemma states.

Lemma 4.2.1. The Godbillon-Vey class $G V(\mathcal{F})$ is independent upon the choice of the 1-forms $\omega$ and $\eta$, used in its definition. Hence it is an invariant of the foliated manifold $(M, \mathcal{F})$.

Proof. The proof is an easy computation. See for instance [17, pag. 250.

The first surprising feature of the Godbillon-Vey class $G V(\mathcal{F})$, coming out directly from its construction, is that it is a 3 -dimensional cohomology class, although it is constructed from the 1-dimensional normal bundle $\nu$ of the foliation. Therefore it can not be a characteristic class of $\nu$.

In fact $G V(\mathcal{F})$ is the first nontrivial example of exotic characteristic classes of the foliation $(M, \mathcal{F})$ (we refer to 12 for a nice and detailed introdution into the theory of these classes).

There exist indeed many examples of transversely oriented codimension 1 foliations for which the Godbillon-Vey class is not zero. The simplest example is 
perhaps that due to Roussarie, which we now briefly describe. For details, we refer to [11, pag. 39-40.

Let $\Sigma_{g}$ be a closed Riemann surface with genus $g>1$. Then its universal covering is the hyperbolic plane $\mathbb{H}^{2}$ and its fundamental group $\Gamma=\pi_{1}\left(\Sigma_{g}\right)$ is a Fuchsian group, acting on $\mathbb{H}^{2}$ by Moebius transformations. Letting now $\Gamma$ act on $\partial \mathbb{H}^{2}=S^{1}$ by Moebius transformations as well, one obtains a foliated bundle $\left(\mathbb{H}^{2} \times_{\Gamma} S^{1}, \mathcal{F}, p\right)$, as shown in Example 1.3 . This foliated bundle is transversely orientable and, once a transverse orientation is chosen, one can show that $\left\langle G V(\mathcal{F}),\left[\mathbb{H}^{2} \times_{\Gamma} S^{1}\right]\right\rangle=4 \pi(1-g)$, which shows that $G V(\mathcal{F}) \in H^{3}\left(\mathbb{H}^{2} \times_{\Gamma} S^{1}, \mathbb{R}\right)$ is nontrivial. Here $\left[\mathbb{H}^{2} \times_{\Gamma} S^{1}\right] \in H_{3}\left(\mathbb{H}^{2} \times_{\Gamma} S^{1}, \mathbb{R}\right)$ is the fundamental class of $\mathbb{H}^{2} \times_{\Gamma} S^{1}$. For the details of this construction and further comments we refer to [11] and [12.

Moreover, among the examples of foliated manifolds whose Godbillon-Vey class is non trivial, there is the family $\left\{\mathcal{F}_{t}\right\}_{t \in \mathbb{R}}$ of transversely oriented codimension 1 foliations on the 3 -sphere due to Thurston [43]. He showed that $\left\langle G V\left(\mathcal{F}_{t}\right),\left[S^{3}\right]\right\rangle=$ $t$ for any $t \in \mathbb{R}$. This proves in particular that there exist foliations whose Godbillon-Vey class has not integral coefficients.

The previous definition of the Godbillon-Vey class as $G V(\mathcal{F})=[\eta \wedge d \eta] \in$ $H^{3}(M, \mathbb{R})$ relies on the Frobenius theorem.

In some cases, however, it is useful to have at the level of forms other representatives for $G V(\mathcal{F})$. For $S^{1}$-foliated bundles Moriyoshi and Natsume [4] found another 3-form, representing the Godbillon-vey class. This form appears in their index formula for the Godbillon-Vey higher index of a leafwise Dirac operator $D=\left(D_{L}\right)_{L \in M / \mathcal{F}}$ on a spin foliation (see again [41]).

The definition of the Godbillon-Vey class does not give any hint about its geometric or dynamical meaning. However, it is nowadays very well known that this class has profound connections both with the geometry and the dynamical properties of the foliation. To illustrate briefly this point, let us mention the two following very deep results.

Theorem 4.2.2. (Duminy) Let $\mathcal{F}$ be a transversely oriented codimension 1 foliation of a closed manifold $M$. If $G V(\mathcal{F}) \neq 0$, then there is an open saturated set of $M$ consisting of leaves with exponential growth.

A detailed proof of this result and further insights in this circle of ideas can be found in [12], Chapter 7.

Theorem 4.2.3. (Hurder and Katok) (33]) Let $\mathcal{F}$ be a transversely oriented codimension 1 foliation of a closed manifold $M$. If $G V(\mathcal{F}) \neq 0$, then the von Neumann algebra $\mathcal{M}(M, \mathcal{F})$ of the foliation has a nontrivial type III component. If $\mathcal{F}$ is ergodic, then $\mathcal{M}(M, \mathcal{F})$ is a type III factor. 


\subsubsection{The Godbillon-Vey class: the Moriyoshi-Natsume' cyclic cocycle}

In this section we will construct the so-called Godbillon-Vey cyclic cocycle as done in [41]. Since they deal with foliated bundles $\left(\tilde{M} \times_{H} T, \mathcal{F}, \pi\right)$, in this section we will focus on this particular class of foliations. Moreover, we will assume that the foliated bundle is of codimension 1 and that all manifolds involved are oriented with the $H$-actions on them preserving the respective orientation.

Thus, consider a foliated bundle $\left(\tilde{M} \times{ }_{H} S^{1}, \mathcal{F}, \pi\right)$, as defined in Example 1.3 . where $S^{1}$ denotes the 1 -dimensional sphere. We set $V=\tilde{M} \times_{H} S^{1}$. Since we are assuming the manifold $\tilde{M}$ to be oriented, the foliation is naturally oriented. Furthermore, since the action of $H$ on $S^{1}$ consists of orientation-preserving diffeomorphisms, it can be checked that the foliation is also transversely oriented. Recall that if the action of the discrete group $H$ on $S^{1}$ is locally free ${ }^{1}$, then there exists a Lie groupoid isomorphism between the holonomy groupoid $H(V, \mathcal{F})$ of $(V, \mathcal{F}, \pi)$ and its monodromy groupoid $\Pi(V, \mathcal{F})=\left(\tilde{M} \times \tilde{M} \times S^{1}\right) / H$. In particular, $H(V, \mathcal{F})$ is Hausdorff.

Through this section we will always assume the $H$-action on $S^{1}$ to be locally free.

Following 41, we will use the pseudodifferential calculus introduced in Section 2.3 .

The Godbillon-Vey cyclic cocycle is the result of the interplay between the transverse orientation of the foliation and the action of the modular automorphism group on the foliation $C^{*}$-algebra $C^{*}(V, \mathcal{F})$. Let us introduce the latter.

Recall that $S^{1}$ is a complete transversal to the foliation and that $H(V, \mathcal{F})_{S^{1}}=$ $\left\{\gamma \in H(V, \mathcal{F}) \mid s(\gamma) \in S^{1}\right\} \cong \tilde{M} \times S^{1}$ (see [41] for details). Now, let $\tilde{\omega}_{\tilde{\tilde{M}_{\tilde{M}}}}$ be a $H$-invariant volume form on $\tilde{M}, \Omega_{\tilde{M} \times S^{1}}$ a $H$-invariant volume form on $\tilde{M} \times S^{1}$ corresponding to the orientation on $V$. Furthermore, let us fix once and for all a volume form on $S^{1}$ which will be denoted by $d t$. Notice that it is impossible in general to choose $d t$ to be $H$-invariant.

There exists a smooth nowhere vanishing function $\psi$ on $\tilde{M} \times S^{1}$ defined by

$$
\tilde{\omega}_{\tilde{M}} \wedge d t=\psi \Omega_{\tilde{M} \times S^{1}}
$$

The function $\psi$ is called a modular function of the holonomy groupoid.

Remark 4.13. It should be noticed that $\psi$ is not compactly supported nor $\Gamma$-invariant. In fact, it holds that

$$
g(\psi)=\mu(g) \psi \quad \forall g \in H
$$

where $\mu(g)$ is a positive smooth function on $S^{1}$ defined by

$$
g \cdot d t=\mu(g) d t \quad \forall g \in H
$$

It can be shown that $\mu(g)$ is 1 -cocycle on $H$ with values in $C^{\infty}\left(S^{1}\right)$.

\footnotetext{
${ }^{1}$ i.e. if for $g \in H$, if there is an open set $U \subseteq S^{1}$ such that $x \cdot g=x$, for all $x \in U$, then $g=e$.
} 
The modular function $\psi$ generates a 1 -parameter group of operators $\left\{\Delta^{i t}\right\}_{t \in \mathbb{R}}$ of $C_{c}^{\infty}\left(\tilde{M} \times S^{1}, \tilde{E}\right)$. Indeed, if $\tilde{E}$ is a $H$-equivariant Hermitian vector bundle over $\tilde{M} \times S^{1}$, the operator $\Delta^{i t}$ is defined by $\Delta^{i t}(\xi)=\psi^{-i t} \xi$ and sends $C_{c}^{\infty}\left(\tilde{M} \times S^{1}, \tilde{E}\right)$ to itself.

One gets the following

Proposition 4.2.4. 1. If $P \in \Psi_{H}^{l}(\tilde{E})$, then $\Delta^{i t} P \Delta^{-i t} \in \Psi_{H}^{l}(\tilde{E})$ for every $l \in \mathbb{Z}$.

2. The operator $\Delta^{i t}$ extends to an intertwining bounded operator on $L_{\tau}^{2}(\tilde{E})$.

3. For all $A \in C^{*}(V, \mathcal{F}, E)$ then $\Delta^{i t} A \Delta^{-i t} \in C^{*}(V, \mathcal{F}, E)$. This defines an automorphism of $C^{*}(V, \mathcal{F}, E)$.

4. Setting $\sigma_{t}(A):=\Delta^{i t} A \Delta^{-i t}$ for any $A \in C^{*}(V, \mathcal{F}, E)$ the family of automorphisms $\sigma_{t}$ is a one-parameter group of automorphisms that leaves the smooth subalgebra $C_{c}^{\infty}(H(\mathcal{F}), E)$ invariant.

For a detailed proof of these results we refer to [41.

Observe that the one-parameter group of automorphisms $\left(\sigma_{t}\right)_{t \in \mathbb{R}}$ on $C^{*}(V, \mathcal{F}, E)$ defines a $\mathbb{R}$-action on the smooth convolution algebra of the foliation.

To construct the Godbillon-Vey cyclic cocycle we just take advantage of this action. The methods used will actually lead to a family of new cyclic cocycles parametrized by closed 1-dimensional Haefliger currents.

For the moment, we assume the bundle $E$ trivial of rank one.

Since the foliation is oriented, we can consider the transverse differential calculus on the holonomy groupoid as defined in Remark 4.2. More precisely, we consider the graded algebra $\Omega^{*}=C_{c}^{\infty}\left(H(\mathcal{F}), \Lambda^{*} t^{*}\left(\nu^{*}\right)\right)$, where $\nu$ denotes the complexification of the normal bundle of the foliation. Moreover, as (complexified) horizontal distribution $Q$ we choose the vector bundle $T_{\text {vert }, \mathbb{C}} V=k e r(d \pi) \otimes \mathbb{C}$, i.e. the complexification of the vertical tangent bundle along the fibers of $\pi$. We denote by $d_{v e r t}$ the corresponding transverse derivation on $\Omega^{*}$.

In the case of a transversely oriented codimension 1 foliation, any horizontal distribution is integrable and therefore $\left(\Omega^{*}, d_{\text {vert }}\right)$ is a differential graded algebra, i.e. $d_{v e r t}^{2}=0$.

Now, we know that the one-parameter group $\left(\sigma_{t}\right)_{t \in \mathbb{R}}$ acts on $\Omega^{0}=C_{c}^{\infty}(H(\mathcal{F}))$. The infinitesimal generator $\delta$ of $\left(\sigma_{t}\right)_{t \in \mathbb{R}}$ defines an unbounded derivation on $C^{*}(V, \mathcal{F})$, whose domain contains $C_{c}^{\infty}(H(\mathcal{F}))$. This derivation, denoted again by $\delta$, is explicitly given by

$$
\delta(K)=[\phi, K]=\phi K-K \phi \quad K \in C_{c}^{\infty}(H(\mathcal{F}))
$$

where $\phi=\log \psi$, i.e. it is the logarithm of the modular function $\psi$.

The commutator can be interpreted in two equivalent ways. First, we can think of $K$ as a compactly smoothing operator through the isomorphism $C_{c}^{\infty}(H(\mathcal{F})) \cong$ $\Psi_{H}^{-\infty}(\mathbb{C})$ and $\phi$ is regarded as a multiplication operator from $C_{c}^{\infty}\left(\tilde{M} \times S^{1}\right)$ to itself. Then the commutator $[\phi, K]$ is the usual commutator of operators. It can checked that it represents a compactly smoothing operator of the foliated 
bundle (see Proposition 4.9 of [41]).

Equivalently, $[\phi, K]$ can be viewed as a compactly supported smooth function on the holonomy groupoid $H(\mathcal{F})$. Indeed, $[\phi, K]$ is a smooth function on $\tilde{M} \times \tilde{M} \times S^{1}$ such that

$[\phi, K](m, n, x)=(\phi K-K \phi)(m, n, x)=\phi(m, x) K(m, n, x)-K(m, n, x) \phi(n, x)$

for all $(m, n, x) \in \tilde{M} \times \tilde{M} \times S^{1}$. $2^{2}$ Moreover it is $H$-invariant (see again Proposition 4.9 of [1]). Thus it yields a smooth function on $H(\mathcal{F})$.

In this way, we have defined a derivation

$$
\delta: C_{c}^{\infty}(H(\mathcal{F})) \rightarrow C_{c}^{\infty}(H(\mathcal{F})) \quad k \mapsto[\phi, k] .
$$

Recall that the space $C_{c}^{\infty}(H(\mathcal{F}))$ is a locally convex vector space carrying its natural LF-topology.

Lemma 4.2.5. The derivation $\delta$ is a continuous operator with respect to the $L F$-topology on $C_{c}^{\infty}(H(\mathcal{F}))$.

Proof. This lemma is a special case of Lemma 4.2 .22 below.

To proceed further, recall that as horizontal distribution we have chosen $Q=T_{\text {vert }, \mathbb{C}} V$. Notice that its pullback $q^{*}\left(T_{\text {vert }, \mathbb{C}} V\right)$ on $\tilde{M} \times S^{1}$ under the quotient map $q: \tilde{M} \times S^{1} \rightarrow V$ is isomorphic to $\tilde{M} \times T_{\mathbb{C}} S^{1}$. The latter is of course the vertical tangent bundle of the bundle $\tilde{M} \times S^{1} \rightarrow \tilde{M}$. With respect to it the de Rham differential $d$ on $\tilde{M} \times S^{1}$ splits as

$$
d=d_{\tilde{M}}+(-1)^{n} d_{S^{1}}
$$

where $d_{\tilde{M}}$ and $d_{S^{1}}$ are respectively the exterior derivatives in the direction of $\tilde{M}$ and of $S^{1}$.

Remark 4.14. It can be shown that the transverse derivation $d_{\text {vert }}$ on $V$ corresponds to $d_{S^{1}}$ under the quotient map $\tilde{M} \times S^{1} \stackrel{q}{\rightarrow} V$, i.e. $q^{*}\left(d_{\text {vert }} k\right)=d_{S^{1}}\left(q^{*} k\right)$, for any $k \in C^{\infty}(V)$.

In the following to simplify the notation, the space of compactly supported smooth sections of $q^{*}\left(T_{\text {vert }, \mathbb{C}}^{*} V\right)=\tilde{M} \times T_{\mathbb{C}}^{*} S^{1}$ will be denoted by $C_{c}^{\infty}(\tilde{M} \times$ $\left.S^{1}, T_{\mathbb{C}}^{*} S^{1}\right)$.

Consider the transverse derivation of $\phi=\log \psi$,

$$
d_{S^{1}} \phi \in C^{\infty}\left(\tilde{M} \times S^{1}, T_{\mathbb{C}}^{*} S^{1}\right)
$$

and think of it as a multiplication operator

$$
d_{S^{1}} \phi: C_{c}^{\infty}\left(\tilde{M} \times S^{1}\right) \rightarrow C_{c}^{\infty}\left(\tilde{M} \times S^{1}, T_{\mathbb{C}}^{*} S^{1}\right)
$$

\footnotetext{
${ }^{2}$ This can be checked by analyzing the Schwartz kernels of the families of operators $\phi K$ and $K \phi$.
} 
by taking into account the $C_{c}^{\infty}\left(\tilde{M} \times S^{1}\right)$-module structure of $C_{c}^{\infty}\left(\tilde{M} \times S^{1}, T_{\mathbb{C}}^{*} S^{1}\right)$. Let $K \in C_{c}^{\infty}(H(\mathcal{F})) \cong \Psi_{H}^{-\infty}(\mathbb{C})$. Then the composition

$$
d_{S^{1}} \phi \circ K: C_{c}^{\infty}\left(\tilde{M} \times S^{1}\right) \rightarrow C_{c}^{\infty}\left(\tilde{M} \times S^{1}, T_{\mathbb{C}}^{*} S^{1}\right) .
$$

makes sense. To define the composition $K \circ d_{S^{1}} \phi$ we observe that

Lemma 4.2.6. There exists an algebra isomorphism

$$
\Theta: \Psi_{H}^{-\infty}(\mathbb{C}) \rightarrow \Psi_{H}^{-\infty}\left(T_{\mathbb{C}}^{*} S^{1}\right)
$$

induced by the chosen transverse volume form $d t \in C^{\infty}\left(\tilde{M} \times S^{1}, T_{\mathbb{C}}^{*} S^{1}\right)$.

Proof. Since $d t$ is a nowhere vanishing transverse form, any section $\xi \in C_{c}^{\infty}(\tilde{M} \times$ $\left.S^{1}, T_{\mathbb{C}}^{*} S^{1}\right)$ can be written as $\xi=f d t$. With this notation the action of $g \in H$ on $\xi$ is expressed by the formula

$$
g \cdot \xi=g \cdot(f d t)=(g \cdot f)(g \cdot d t)=\mu(g)(g \cdot f) d t .
$$

Now, on the trivial bundle $\left(\tilde{M} \times S^{1}\right) \times \mathbb{C}$ over $\tilde{M} \times \mathbb{C}$ define a $H$-action by

$$
\mathbb{C}_{\left(m^{\prime}, t\right)} \ni\left(m^{\prime}, t, v\right) \mapsto\left(m^{\prime} g, t g, \mu(g)^{-1}(t) v\right) \in \mathbb{C}_{\left(m^{\prime} g, t g\right)} \quad g \in H
$$

Denote the corresponding $H$-equivariant bundle by $\widetilde{\mathbb{C}}$.

Then the corresponding action on a section $\sigma \in C_{c}^{\infty}\left(\tilde{M} \times S^{1}, \widetilde{\mathbb{C}}\right)$ is

$$
(g \cdot \sigma)\left(m^{\prime}, t\right)=\mu(g)(t) \sigma\left(m^{\prime} g, t g\right) \quad \forall\left(m^{\prime}, t\right) \in \tilde{M} \times S^{1}
$$

and we get the $H$-equivariant isomorphism

$$
j: C_{c}^{\infty}\left(\tilde{M} \times S^{1}, \widetilde{\mathbb{C}}\right) \rightarrow C_{c}^{\infty}\left(\tilde{M} \times S^{1}, T_{\mathbb{C}}^{*} S^{1}\right) .
$$

defined by

$$
f \mapsto f d t .
$$

Hence, for any $P \in \Psi_{H}^{-\infty}(\widetilde{\mathbb{C}})$ we can define $P^{\prime} \in \Psi_{H}^{-\infty}\left(T_{\mathbb{C}}^{*} S^{1}\right)$ by setting $P^{\prime}=$ $j P j^{-1}$. This correspondence defines an algebra isomorphism between $\Psi_{H}^{-\infty}(\widetilde{\mathbb{C}})$ and $\Psi_{H}^{-\infty}\left(T_{\mathbb{C}}^{*} S^{1}\right)$.

Moreover, as proved in [41, there is a natural isomorphism $i$ from $\Psi_{H}^{-\infty}(\mathbb{C})$ onto $\Psi_{H}^{-\infty}(\widetilde{\mathbb{C}})$. Composing these two bijections, we get the desired isomorphism.

Thus, unless otherwise specified, we can identify $\Psi_{H}^{-\infty}(\mathbb{C})$ with $\Psi_{H}^{-\infty}\left(T_{\mathbb{C}}^{*} S^{1}\right)$.

Remark 4.15. Passing to Schwartz kernels, by Lemma 4.2.6 we get an algebra isomorphism $C_{c}^{\infty}(H(\mathcal{F})) \stackrel{\cong}{\rightrightarrows} C_{c}^{\infty}\left(H(\mathcal{F}), E N D\left(T_{\text {vert }, \mathbb{C}}^{*} V\right)\right)$, which is more explicitly determined as follows.

Consider the transverse volume form $d t \in C_{c}^{\infty}\left(\tilde{M} \times S^{1}, T_{\mathbb{C}}^{*} S^{1}\right)$ and the corresponding section $X \in C_{c}^{\infty}\left(\tilde{M} \times S^{1}, T_{\mathbb{C}} S^{1}\right)$ such that $d t(X) \equiv 1$. If $K=$ $\left(K_{t}\right)_{t \in S^{1}} \in \Psi_{H}^{-\infty}(\mathbb{C})$ and $\xi=f d t \in C_{c}^{\infty}\left(\tilde{M} \times S^{1}, T_{\mathbb{C}}^{*} S^{1}\right)$, then

$$
\Theta(K)(f d t)=(K(f)) d t .
$$


If we forget for a moment the $H$-invariance of the family of operators $K=$ $\left(K_{t}\right)_{t \in S^{1}}$, then its Schwartz kernel is a smooth function $k \in C^{\infty}\left(\tilde{M} \times \tilde{M} \times S^{1}\right)$ and the isomorphism $\Theta$ sends

$$
k \mapsto k\left(\hat{s}^{*}(X) \otimes \hat{t}^{*}(d t)\right) \in C^{\infty}\left(\tilde{M} \times \tilde{M} \times S^{1}, \hat{s}^{*}\left(T_{\mathbb{C}} S^{1}\right) \otimes \hat{t}^{*}\left(T_{\mathbb{C}}^{*} S^{1}\right)\right),
$$

where $\hat{s}(m, n, t)=(n, t)$ is the source map of the groupoid $\tilde{M} \times \tilde{M} \times S^{1}$ and $\hat{t}(m, n, t)=(m, t)$ its target map.

By definition $\left(\hat{s}^{*}(X) \otimes \hat{t}^{*}(d t)\right) \in C^{\infty}\left(\tilde{M} \times \tilde{M} \times S^{1}, \hat{s}^{*}\left(T_{\mathbb{C}} S^{1}\right) \otimes \hat{t}^{*}\left(T_{\mathbb{C}}^{*} S^{1}\right)\right)$ is a nowhere vanishing section and it is easily seen to be $H$-invariant. Hence, since $k \in C^{\infty}\left(\tilde{M} \times \tilde{M} \times S^{1}\right)$ is $H$-invariant, then $k\left(\hat{s}^{*}(X) \otimes \hat{t}^{*}(d t)\right)$ is so as well.

In other words, since $\left(\hat{s}^{*}(X) \otimes \hat{t}^{*}(d t)\right)$ is $H$-invariant, it descends to a section $\mu \in C^{\infty}\left(H(\mathcal{F}), E N D\left(T_{\text {vert }, \mathbb{C}}^{*}\right)\right)$ and for all $k \in C_{c}^{\infty}(H(\mathcal{F}))$, one has $\Theta(k)=$ $k \mu \in C_{c}^{\infty}\left(H(\mathcal{F}), E N D\left(T_{\text {vert }, \mathbb{C}}^{*} V\right)\right)$.

The lemma above make possible to define the commutator

$$
\left[d_{S^{1}} \phi, K\right]=d_{S^{1}} \phi \circ K-K \circ d_{S^{1}} \phi \quad K \in C_{c}^{\infty}(H(\mathcal{F}))
$$

Lemma 4.2.7. One has $\left[d_{S^{1}} \phi, K\right] \in C_{c}^{\infty}\left(H(\mathcal{F}), t^{*}\left(T_{\text {vert }, \mathbb{C}}^{*} V\right)\right)$.

Proof. To show the claim, let us first think in terms of operators. Thus, let us take $K \in \Psi_{H}^{-\infty}(\mathbb{C})$. Since $d_{S^{1}} \phi \in C^{\infty}\left(\tilde{M} \times S^{1}, T_{\mathbb{C}}^{*} S^{1}\right)$, the operators $d_{S^{1}} \phi \circ K$ and $K \circ d_{S^{1}} \phi$ are families of smoothing operators from $C_{c}^{\infty}\left(\tilde{M} \times S^{1}\right)$ to $C_{c}^{\infty}(\tilde{M} \times$ $\left.S^{1}, T_{\mathbb{C}}^{*} S^{1}\right)$ with $H$-compact support. Then, we have only to check that the commutator $\left[d_{S^{1}} \phi, K\right]$ is $H$-invariant.

Using the transverse volume form $d t$ on $\tilde{M} \times S^{1}$, we can write $d_{S^{1}} \phi=\alpha d t$. For $g \in H$ one has that

$$
\begin{gathered}
g\left(d_{S^{1}} \phi\right)=g(\alpha d t)=\mu(g) g(\alpha) d t= \\
d_{S^{1}} g(\phi)=d_{S^{1}}(\phi+\log \mu(g))=(\alpha+\rho(g)) d t,
\end{gathered}
$$

where $\rho(g) d t=d_{S^{1}} \log \mu(g)$ is a smooth function constant along $\tilde{M}_{x}$, for all $x \in S^{1}$. Therefore, $\mu(g) g(\alpha)=\alpha+\rho(g)$.

Then

$$
\begin{gathered}
g\left(d_{S^{1}} \phi \circ K-K \circ d_{S^{1}}\right)=g\left(d_{S^{1}} \phi\right) g K-g\left(K d_{S^{1}} \phi\right)= \\
=g\left(d_{S^{1}} \phi\right) K g-K g\left(d_{S^{1}} \phi\right) g=(g(\alpha d t) K-K g(\alpha d t)) g= \\
=(\mu(g) g(\alpha) K d t-K(\mu(g) g(\alpha)) d t) g=((\alpha+\rho(g)) K d t-K(\alpha+\rho(g)) d t) g .
\end{gathered}
$$

Now, since $\rho(g)$ is constant along $\tilde{M}_{x}$ it commutes with $K$ and hence we obtain

$$
\begin{gathered}
((\alpha+\rho(g)) K d t- \\
=\left(d_{S^{1}} \phi \circ K-K \circ d_{S^{1}} \phi\right) g .
\end{gathered}
$$

Thus, $\left[d_{S^{1}} \phi, K\right] \in \Psi_{H}^{-\infty}\left(\mathbb{C}, T_{\mathbb{C}}^{*} S^{1}\right)$. Finally, using the isomorphism given by passing to Schwartz kernels

$$
\Phi: \Psi_{H}^{-\infty}\left(\mathbb{C}, T_{\mathbb{C}}^{*} S^{1}\right) \cong C_{c}^{\infty}\left(H(\mathcal{F}), t^{*}\left(T_{\text {vert }, \mathbb{C}}^{*} V\right)\right)
$$

we get a smooth section in the chosen horizontal distribution, proving the lemma. 
According to the above lemma, we have the map

$$
\left[d_{S^{1}} \phi, \cdot\right]: C_{c}^{\infty}(H(\mathcal{F})) \rightarrow C_{c}^{\infty}\left(H(\mathcal{F}), t^{*}\left(T_{\text {vert }, \mathbb{C}}^{*} V\right)\right)
$$

Notice that the space $C_{c}^{\infty}\left(H(\mathcal{F}), t^{*}\left(T_{\text {vert }, \mathbb{C}} V\right)\right)$ is a bimodule over $C_{c}^{\infty}(H(\mathcal{F}))$, with bimodule-structure given by the graded product of $\Omega^{*}$, i.e. by convolution. On the other side, $\Psi_{H}^{-\infty}\left(\mathbb{C}, T_{\mathbb{C}}^{*} S^{1}\right)$ has also a well-defined bimodule structure over $C_{c}^{\infty}(H(\mathcal{F})) \cong \Psi_{H}^{-\infty}(\mathbb{C})$, defined through the isomorphism 4.2.6.

Lemma 4.2.8. The linear isomorphism

$$
\Phi: \Psi_{H}^{-\infty}\left(\mathbb{C}, T_{\mathbb{C}}^{*} S^{1}\right) \cong C_{c}^{\infty}\left(H(\mathcal{F}), t^{*}\left(T_{\text {vert }, \mathbb{C}}^{*} V\right)\right)
$$

defined by associating to each operator $P$ its corresponding Schwartz kernel $p$, is an isomorphism of bimodules.

Proof. The right action of $C_{c}^{\infty}(H(\mathcal{F})) \cong \Psi_{H}^{-\infty}(\mathbb{C})$ on $\Psi_{H}^{-\infty}\left(\mathbb{C}, T_{\mathbb{C}}^{*} S^{1}\right)$ is defined to be the composition $P \circ K \in \Psi_{H}^{-\infty}\left(\mathbb{C}, T_{\mathbb{C}}^{*} S^{1}\right)$ for every element $P \in$ $\Psi_{H}^{-\infty}\left(\mathbb{C}, T_{\mathbb{C}}^{*} S^{1}\right)$ and $K \in \Psi_{H}^{-\infty}(\mathbb{C})$. By passing to the Schwartz kernels $p \in$ $C_{c}^{\infty}\left(H(\mathcal{F}), t^{*}\left(T_{\text {vert }, \mathbb{C}}^{*} V\right)\right)$ and $k \in C_{c}^{\infty}(H(\mathcal{F}))$ of, respectively, $P$ and $K$ we have that composition of operators coincides with the convolution $p * k \in$ $C_{c}^{\infty}\left(H(\mathcal{F}), t^{*}\left(T_{\text {vert }, \mathbb{C}}^{*} V\right)\right)$, which is exactly the right action of $C_{c}^{\infty}(H(\mathcal{F}))$ on $C_{c}^{\infty}\left(H(\mathcal{F}), t^{*}\left(T_{\text {vert }, \mathbb{C}}^{*} V\right)\right)$.

Hence, we get that $\Phi$ is a morphism of right $C_{c}^{\infty}(H(\mathcal{F}))$-modules.

To show that it preserves the left actions as well, we argue in the following way. By definition, the left action of an element $K \in \Psi_{H}^{-\infty}(\mathbb{C})$ on $P \in \Psi_{H}^{-\infty}\left(\mathbb{C}, T_{\mathbb{C}}^{*} S^{1}\right)$ is given by

$$
K \cdot P=\Theta(K) \circ P .
$$

In terms of the Schwartz kernels $k$ and $p$ of $K$ and $P$, respectively, we deduce from Remark 4.15 that the above formula takes the form

$$
k \cdot p(\gamma)=\int_{H(\mathcal{F})^{t(\gamma)}} k\left(\gamma_{1}\right) \mu\left(\gamma_{1}\right) p\left(\gamma_{1}^{-1} \gamma\right) d \gamma_{1}
$$

for all $\gamma \in H(\mathcal{F})$.

On the other side, recall from Section 4.1.2 that the left action of $C_{c}^{\infty}(H(\mathcal{F}))$ on $C_{c}^{\infty}\left(H(\mathcal{F}), t^{*}\left(T_{v e r t, \mathbb{C}}^{*} V\right)\right)$ is defined by means of the holonomy action through the formula (we have slightly changed notation but the meaning is the same)

$$
k \cdot p(\gamma)=\int_{H(\mathcal{F})^{t(\gamma)}} k\left(\gamma_{1}\right) \lambda\left(\gamma_{1}\right) p\left(\gamma_{1}^{-1} \gamma\right) d \gamma_{1} .
$$

Notice that the holonomy action $\lambda$ on the normal bundle can be viewed as a section $\lambda \in C^{\infty}\left(H(\mathcal{F}), E N D\left(T_{\text {vert }, \mathbb{C}}^{*} V\right)\right)$ such that $\lambda\left(\gamma_{1} \gamma_{2}\right)=\lambda\left(\gamma_{1}\right) \lambda\left(\gamma_{2}\right)$ for all $\gamma_{1}, \gamma_{2} \in H(\mathcal{F})$.

Hence, if we prove that $\mu=\lambda$, then the left actions will coincide.

Now, since both $\lambda$ and $\mu$ belong to $C^{\infty}\left(H(\mathcal{F}), E N D\left(T_{\text {vert }, \mathbb{C}}^{*} V\right)\right)$ we can consider the liftings $\hat{\lambda}$ of $\lambda$ and $\left(\hat{s}^{*}(X) \otimes \hat{t}^{*}(d t)\right)$ of $\mu$ on the space of smooth sections $C^{\infty}\left(\tilde{M} \times \tilde{M} \times S^{1}, \hat{s}^{*}\left(T_{\mathbb{C}} S^{1}\right) \otimes \hat{t}^{*}\left(T_{\mathbb{C}}^{*} S^{1}\right)\right)$. 
It is not difficult to see that $\hat{\lambda}$ is exactly the holonomy action on the bundle $\tilde{M} \times T_{\mathbb{C}}^{*} S^{1}$ over $\tilde{M} \times S^{1}$. Moreover, since the transverse volume form $d t$ is constant along the leaves $\tilde{M}_{t}$ of the trivial foliation $\mathcal{F}_{0}=\left(\tilde{M}_{t}\right)_{t \in S^{1}}$ on $\tilde{M} \times S^{1}$, we deduce that it is holonomy-invariant. Since $d t$ vanishes nowhere and it is in each point $(m, t) \in \tilde{M} \times S^{1}$ a base of the fiber $\left.\left(\tilde{M} \times T_{\mathbb{C}}^{*} S^{1}\right)\right)_{(m, t)}$, one deduces that $\hat{\lambda}=\left(\hat{s}^{*}(X) \otimes \hat{t}^{*}(d t)\right)$, which in turn implies the equality $\lambda=\mu$. This concludes the proof.

Thus we get

Proposition 4.2.9. The commutator

$$
\left[d_{S^{1}} \phi, \cdot\right]: C_{c}^{\infty}(H(\mathcal{F})) \rightarrow C_{c}^{\infty}\left(H(\mathcal{F}), t^{*}\left(T_{\text {vert }, \mathbb{C}} V\right)\right)
$$

defines a bimodule derivation, that is continuous with respect to the LF-topologies on $C_{c}^{\infty}(H(\mathcal{F}))$ and on $C_{c}^{\infty}\left(H(\mathcal{F}), t^{*}\left(T_{\text {vert }, \mathbb{C}} V\right)\right)$.

Proof. The fact that $\left[d_{S^{1}} \phi, \cdot\right]$ defines a bimodule derivation follows directly from the discussion above. For the continuity we refer to Corollary 4.2.25.

In what follows, we will denote the derivation $\left[d_{S^{1}} \phi, \cdot\right]$ by $\delta_{1}$.

There is also a linear map $\delta^{\prime}$ defined on the bimodule $C_{c}^{\infty}\left(H(\mathcal{F}), t^{*}\left(T_{\text {vert }, \mathbb{C}}^{*} V\right)\right)$ which is important to consider. It is defined as follows. Using the isomorphism 4.54 and the multiplication operator $\Delta^{i t}$ on both the trivial bundle $\left(\tilde{M} \times S^{1}\right) \times \mathbb{C}$ and on $\tilde{M} \times T_{\mathbb{C}}^{*} S^{1}$, one has

Lemma 4.2.10. There is a one-parameter group $\left(\tilde{\sigma}_{t}\right)_{t \in \mathbb{R}}$ of automorphisms of $\Psi_{H}^{-\infty}\left(\mathbb{C}, T_{\mathbb{C}}^{*} S^{1}\right)$, that is given by

$$
\tilde{\sigma}_{t}(P)=\Delta^{i t} P \Delta^{-i t}
$$

for any $P \in \Psi_{H}^{-\infty}\left(\mathbb{C}, T_{\mathbb{C}}^{*} S^{1}\right)$.

Proof. The only thing to prove is that $\Delta^{i t} P \Delta^{-i t}$ is $H$-invariant. But this follows from a straightforward computation.

The linear map $\delta^{\prime}$ is now defined to be the infinitesimal generator of the one-parameter group $\left(\tilde{\sigma}_{t}\right)_{t \in \mathbb{R}}$. Hence using the isomorphism 4.54

$$
\delta^{\prime}(P)=[\phi, P] \quad P \in C_{c}^{\infty}\left(H(\mathcal{F}), t^{*}\left(T_{\text {vert }, \mathbb{C}}^{*} V\right)\right),
$$

where $\phi=\log \psi$. This map is continuous with respect to the LF-topology on $C_{c}^{\infty}\left(H(\mathcal{F}), t^{*}\left(T_{\text {vert }, \mathbb{C}}^{*} V\right)\right)$.

The importance of introducing the map $\delta^{\prime}$ relies on the following result.

Lemma 4.2.11. For every $K \in C_{c}^{\infty}(H(\mathcal{F})), \delta_{1}(\delta(K))=\delta^{\prime}\left(\delta_{1}(K)\right)$.

Proof. It is a direct consequence of the definitions and of the Jacobi identity. 
Remark 4.16. Summarizing, we have constructed two derivations on $\Omega^{*}$. The first one is the derivation $\delta_{\Omega}^{0}$ of degree 0 defined on $\Omega^{0}$ by

$$
\delta_{\Omega}^{0}(k)=\delta(k)=[\phi, k]=\phi k-k \phi \quad k \in \Omega^{0}
$$

and on $\Omega^{1}$ by

$$
\delta_{\Omega}^{0}(p)=\delta^{\prime}(p) \quad p \in \Omega^{1}
$$

The other one is the derivation $\delta_{\Omega}^{1}$ of degree 1 on $\Omega^{*}$ given by the formula

$$
\delta_{\Omega}^{1}(k)=\delta_{1}(k)=\left[d_{S^{1}} \phi, k\right]
$$

for $k \in \Omega^{0}$, and $\delta_{\Omega}^{1}(p)=0$, for $p \in \Omega^{1}$.

Now, on the graded algebra $\Omega^{*}$ we have natural graded traces $\int_{C}$ for any closed holonomy-invariant current $C \in C_{*}(\operatorname{Tr} \mathcal{F})$ (see Lemma 4.1.3).

In particular, any closed $C \in C_{1}(\operatorname{Tr} \mathcal{F})$ defines a so-called trace map

$$
\int_{C}: C_{c}^{\infty}\left(H(\mathcal{F}), t^{*}\left(T_{\text {vert }, \mathbb{C}}^{*} V\right)\right) \rightarrow \mathbb{C}
$$

such that

$$
\int_{C}\left(k_{0} k_{1}\right)=\int_{C}\left(k_{1} k_{0}\right) \quad \text { for } k_{i} \in \Omega^{i} .
$$

Lemma 4.2.12. 1. One has

$$
\int_{C} \delta_{\Omega}^{1}=0
$$

for all closed $C \in C_{1}(\operatorname{Tr} \mathcal{F})$.

2. Similarly

$$
\int_{C} \delta_{\Omega}^{0}=0
$$

for all closed $C \in C_{1}(\operatorname{Tr} \mathcal{F})$.

Proof. 1). It suffices to show that $\int_{C} \delta_{1}=0$. Notice first that each $C \in C_{1}(\operatorname{Tr} \mathcal{F})$ induces a $H$-invariant current on $S^{1}$, which we denote by the same symbol. If $\mathcal{D}$ denotes a fundamental domain for the free $H$-action on $\tilde{M}$, one has that

$$
\left\langle C, \int_{\mathcal{F}}\left[d_{S^{1}} \phi, k\right]_{\mid V}\right\rangle=\left\langle C, \int_{\mathcal{D}}\left[d_{S^{1}} \phi, k\right](m, m, x) d m\right\rangle .
$$

Now, for any $x \in S^{1}$ it holds

$$
\int_{\mathcal{D}}\left[d_{S^{1}} \phi, k\right](m, m, x) d m=0 .
$$

which immediately implies

$$
\int_{C} \delta_{\Omega}^{1}=0
$$

2 ). The proof is the same as above.

Therefore, we have 
Lemma 4.2.13. The following formula

$$
\dot{\tau}_{C}\left(k_{0}, k_{1}\right)=\int_{C} k_{0} \delta_{1}\left(k_{1}\right)
$$

defines a continuous cyclic 1 -cocycle on $\Omega^{0}=C_{c}^{\infty}(H(\mathcal{F}))$.

The cocycles $\dot{\tau}_{C}$ are closely related to the cocycles $\tau_{C}$ as given by Theorem 4.1.4 More precisely

Proposition 4.2.14. Let $C \in C_{1}(T r \mathcal{F})$ closed and let $\tau_{C}$ be the corresponding cyclic 1-cocycle as defined by Theorem 4.1.4. Then the following formula holds

$$
\dot{\tau}_{C}\left(k_{0}, k_{1}\right)=\lim _{t \rightarrow 0} \frac{1}{t}\left(\tau_{C}\left(\sigma_{t}\left(k_{0}\right), \sigma_{t}\left(k_{1}\right)\right)-\tau_{C}\left(k_{0}, k_{1}\right)\right)
$$

where $\left(\sigma_{t}\right)_{t \in \mathbb{R}}$ is the modular group on $C_{c}^{\infty}(H(\mathcal{F}))$.

Before proving the proposition, let us make some observation. Consider $\left(\Omega^{*}, d_{v e r t}\right)$ and recall that $d_{v e r t}^{2}=0$, since we are dealing with a transversely oriented codimension 1 foliation. Thus, $\left(\Omega^{*}, d_{\text {vert }}\right)$ is a DGA.

Further, since $H(\mathcal{F}) \cong \frac{\tilde{M} \times \tilde{M} \times S^{1}}{H}$, each element $k \in \Omega^{0}=C_{c}^{\infty}(H(\mathcal{F}))$ can be thought of as a smooth $H$-invariant function. We shall denote the function on $H(\mathcal{F})$ and its lift on $\tilde{M} \times \tilde{M} \times S^{1}$ by the same symbol.

If $q: \tilde{M} \times \tilde{M} \times S^{1} \rightarrow\left(\tilde{M} \times \tilde{M} \times S^{1}\right) / H$ is the quotient map, it is immediate to see that

$$
q^{*}\left(t^{*}\left(T_{\text {vert }, \mathbb{C}} V\right)\right) \cong \tilde{M} \times \tilde{M} \times T_{\mathbb{C}}^{*} S^{1} .
$$

This is of course the horizontal distribution complementary to the obvious foliation $\mathcal{F}_{0}=\{\tilde{M} \times \tilde{M} \times\{t\}\}_{t \in S^{1}}$ on $\tilde{M} \times \tilde{M} \times S^{1}$. The foliation $\mathcal{F}_{0}$ corresponds clearly to that on the holonomy groupoid under $q$. We shall denote $d_{S^{1}}$ the corresponding transverse derivation on $\tilde{M} \times \tilde{M} \times S^{1}$.

Given any closed $C \in C_{1}(\operatorname{Tr} \mathcal{F})$ the corresponding cyclic cocycle $\tau_{C}$ can be written as

$$
\tau_{C}\left(k_{0}, k_{1}\right)=\left\langle C, \int_{\mathcal{D}} \int_{\tilde{M}} k_{0}(m, n, x)\left(d_{S^{1}} k_{1}\right)(n, m, x) d n d m,\right\rangle
$$

where $\mathcal{D}$ denotes a fundamental domain for the action of $H$ on $\tilde{M}$, the function $k_{1}$ is regarded as $H$-invariant functions on $\tilde{M} \times \tilde{M} \times S^{1}$, while $k_{0}$ is regarded as the corresponding unique image through the isomorphism 4.2.6. Finally we have used the transverse derivation $d_{S^{1}}$ on $\tilde{M} \times \tilde{M} \times S^{1}$.

Let us now prove Proposition 4.2 .14

Proof. Recall that the infinitesimal generator of the modular group $\left(\sigma_{t}\right)$ is precisely the derivation $\delta$ on $C_{c}^{\infty}(H(\mathcal{F}))$. Now,

$$
\begin{gathered}
\lim _{t \rightarrow 0} \frac{1}{t}\left(\tau_{C}\left(\sigma_{t}\left(k_{0}\right), \sigma_{t}\left(k_{1}\right)\right)-\tau_{C}\left(k_{0}, k_{1}\right)\right)=\tau_{C}\left(\delta\left(k_{0}\right), k_{1}\right)+\tau_{C}\left(k_{0}, \delta\left(k_{1}\right)\right)= \\
=\tau_{C}\left(\left[\phi, k_{0}\right], k_{1}\right)+\tau_{C}\left(k_{0},\left[\phi, k_{1}\right]\right)=
\end{gathered}
$$


$\left\langle C, \int_{\mathcal{D}} \int_{\tilde{M}}\left[\phi, k_{0}\right](m, n, x)\left(d_{S^{1}} k_{1}\right)(n, m, x) d n+k_{0}(m, n, x) d_{S^{1}}\left(\left[\phi, k_{1}\right]\right)(n, m, x) d n d m\right\rangle$.

Let us study the integrand of this expression. One has

$$
\begin{gathered}
{\left[\phi, k_{0}\right](m, n, x)\left(d_{S^{1}} k_{1}\right)(n, m, x)+k_{0}(m, n, x) d_{S^{1}}\left(\left[\phi, k_{1}\right]\right)(n, m, x)=} \\
=\left(\phi k_{0}-k_{0} \phi\right)(m, n, x)\left(d_{S^{1}} k_{1}\right)(n, m, x)+k_{0}(m, n, x)\left(d_{S^{1}}\left(\phi k_{1}-k_{1} \phi\right)\right)(n, m, x)= \\
=\left(\phi(m, x) k_{0}(m, n, x)-k_{0}(m, n, x) \phi(n, x)\right)\left(d_{S^{1}} k_{1}\right)(n, m, x)+ \\
k_{0}(m, n, x)\left(d_{S^{1}}(\phi) k_{1}+\phi d_{S^{1}}\left(k_{1}\right)-d_{S^{1}}\left(k_{1}\right) \phi-k_{1} d_{S^{1}}(\phi)\right)(n, m, x)= \\
=k_{0}(m, n, x)\left(d_{S^{1}}(\phi)(n, x) k_{1}(n, m, x)\right)-k_{0}(m, n, x)\left(k_{1}(n, m, x) d_{S^{1}}(\phi)(m, x)\right)= \\
=k_{0}(m, n, x)\left[d_{S^{1}} \phi, k_{1}\right](n, m, x) .
\end{gathered}
$$

Hence, we have obtained that

$$
\begin{gathered}
\left\langle C, \int_{\mathcal{D}} \int_{\tilde{M}}\left[\phi, k_{0}\right](m, n, x)\left(d_{S^{1}} k_{1}\right)(n, m, x) d n d m+k_{0}(m, n, x) d_{S^{1}}\left(\left[\phi, k_{1}\right]\right)(n, m, x) d n d m\right\rangle= \\
\left\langle C, \int_{\mathcal{D}} \int_{\tilde{M}} k_{0}(m, n, x)\left[d_{S^{1}} \phi, k_{1}\right](n, m, x) d n d m\right\rangle= \\
=\int_{C} k_{0} \delta_{1}\left(k_{1}\right) .
\end{gathered}
$$

This finishes the proof.

In other words, we have built out of the cocycles $\tau_{C}$ a new family of cyclic cocycles $\dot{\tau}_{C}$ as the "Lie derivative" of $\tau_{C}$ under the flow $\left(\sigma_{t}\right)_{t \in \mathbb{R}}$. A remarkable property of the cocycles $\dot{\tau}_{C}$ is that they are invariant under the action of the modular group. Indeed

Proposition 4.2.15. One has that

$$
\ddot{\tau}_{C}\left(k_{0}, k_{1}\right)=\lim _{t \rightarrow 0} \frac{1}{t}\left(\dot{\tau}_{C}\left(\sigma_{t}\left(k_{0}\right), \sigma_{t}\left(k_{1}\right)\right)-\dot{\tau}_{C}\left(k_{0}, k_{1}\right)\right)=0 .
$$

Proof. Using the properties of the derivations established above, one has

$$
\begin{gathered}
\lim _{t \rightarrow 0} \frac{1}{t}\left(\dot{\tau}_{C}\left(\sigma_{t}\left(k_{0}\right), \sigma_{t}\left(k_{1}\right)\right)-\dot{\tau}_{C}\left(k_{0}, k_{1}\right)\right)=\dot{\tau}_{C}\left(\delta\left(K_{0}\right), k_{1}\right)+\dot{\tau}_{C}\left(k_{0} \delta\left(k_{1}\right)\right)= \\
=\int_{C}\left(\delta\left(k_{0}\right) \delta_{1}\left(k_{1}\right)\right)+\int_{C}\left(k_{0} \delta_{1}\left(\delta\left(k_{1}\right)\right)\right)= \\
=\int_{C}\left(\delta\left(k_{0}\right) \delta_{1}\left(k_{1}\right)\right)+\int_{C}\left(k_{0} \delta^{\prime}\left(\delta_{1}\left(k_{1}\right)\right)\right)= \\
=\int_{C}\left(\delta\left(k_{0}\right) \delta_{1}\left(k_{1}\right)\right)+\int_{C}\left(\delta^{\prime}\left(k_{0} \delta_{1}\left(k_{1}\right)\right)\right)-\int_{C}\left(\delta\left(k_{0}\right) \delta_{1}\left(k_{1}\right)\right)=0 .
\end{gathered}
$$


As already mentioned in Chapter 3 , once one has a cyclic $n$-cocycle $\tau$ that is invariant under the action of a one-parameter group $\left(\alpha_{t}\right)_{t \in \mathbb{R}}$, it is always possible to define a cyclic $(n+1)$-cocycle $i_{D}(\tau)$, called the contraction of $\tau$ with respect to the infinitesimal generator $D$ of $\left(\alpha_{t}\right)_{t \in \mathbb{R}}$.

Its explicit definition is given in Equation 4.81 below and we refer to [17], pag. 264 for details.

In our case, to any closed holonomy-invariant current $C \in C_{1}(\operatorname{Tr} \mathcal{F})$ we can associate a cyclic $2-$ cocycle $\mathbf{g v}_{C}$ on $C_{c}^{\infty}(H(\mathcal{F}))$, namely the contraction of $\dot{\tau}_{C}$ with respect to the one-parameter group $\left(\sigma_{t}\right)_{t \in \mathbb{R}}$.

Definition 4.6. The cyclic 2-cocycle $\mathbf{g v}_{C}$ on $C_{c}^{\infty}(H(\mathcal{F}))$ associated to $C \in$ $C_{1}(\operatorname{Tr} \mathcal{F})$ is called the distributional $C$-Godbillon-Vey cyclic cocycle of the foliated bundle $\left(\tilde{M} \times_{H} T, \mathcal{F}, \pi\right)$.

Each $C$-Godbillon-Vey cyclic cocycle $\mathbf{g v}_{C}$ is a continuous cyclic 2-cocycle on $C_{c}^{\infty}(H(\mathcal{F}))$.

Recall that each $H$-invariant current on $S^{1}$ gives rise to a holonomy invariant current on $(V, \mathcal{F})$.

Notice that if the closed holonomy-invariant current $C \in C_{1}(\operatorname{Tr} \mathcal{F})$ corresponds to the measure determined by the transverse volume form $d t$, then the corresponding Godbillon-Vey cyclic cocycle gv is the cyclic 2-cocycle constructed in 41 .

Let us now analyze the situation where the bundle $E$ is a nontrivial bundle. In this case, the infinitesimal generator $\delta$ of the one-parameter group $\left(\sigma_{t}\right)_{t \in \mathbb{R}}$ gives rise to a derivation on the algebra $C_{c}^{\infty}(H(\mathcal{F}), E) \cong \Psi_{H}^{-\infty}(\tilde{E})$.

Moreover, one considers the bimodule

$$
\Omega_{E}^{1}=C_{c}^{\infty}\left(H(\mathcal{F}), E N D(E) \otimes t^{*}\left(\left(T_{\text {vert }, \mathbb{C}} V\right)\right)\right) \cong \Psi_{H}^{-\infty}\left(\tilde{E}, \tilde{E} \otimes\left(\tilde{M} \times T_{\mathbb{C}}^{*} S^{1}\right)\right)
$$

over $C_{c}^{\infty}(H(\mathcal{F}), E)$. Equivalently, the bimodule $\Omega_{E}^{1}$ can be viewed as the space of operators $\Psi_{H}^{-\infty}\left(\tilde{E}, \tilde{E} \otimes\left(\tilde{M} \times T_{\mathbb{C}}^{*} S^{1}\right)\right)$, which is a bimodule over $\Psi_{H}^{-\infty}(\tilde{E})$. To define the left action one proves that there is an algebra isomorphism

$$
\Psi_{H}^{-\infty}(\tilde{E}) \cong \Psi_{H}^{-\infty}\left(\tilde{E} \otimes\left(\tilde{M} \times T_{\mathbb{C}}^{*} S^{1}\right)\right)
$$

generalizing Lemma 4.2.6.

The transverse derivation $d_{S^{1}} \phi$ induces again a multiplication operator

$$
d_{S^{1}} \phi: C_{c}^{\infty}\left(\tilde{M} \times S^{1}, \tilde{E}\right) \rightarrow C_{c}^{\infty}\left(\tilde{M} \times S^{1}, \tilde{E} \otimes\left(\tilde{M} \times T_{\mathbb{C}}^{*} S^{1}\right)\right)
$$

exactly as above and one can show that the commutator $\left[d_{S^{1}} \phi, K\right] \in \Omega_{E}^{1}$, for all $K \in C_{c}^{\infty}(H(\mathcal{F}), E)$. This defines the bimodule derivation $\delta_{1}: \Omega_{E}^{0} \rightarrow \Omega_{E}^{1}$.

One also gets the linear map $\delta^{\prime}$ exactly as in the scalar case.

Finally, for any $C \in C_{1}(\operatorname{Tr}(\mathcal{F}))$ one has a graded trace $\int_{C}$ on $\Omega_{E}^{1}$, which happens to be closed with respect to the derivation $\delta_{1}$ and with respect to the linear map $\delta^{\prime}$. The proof of these results follows the same lines done in the scalar case.

Hence, with these ingredients we can define on $C_{c}^{\infty}(H(\mathcal{F}), E)$ the family of cyclic $1-$ cocycles $\dot{\tau}_{C}$ and the distributional Godbillon-Vey cyclic cocycles $\mathbf{g v}_{C}$ 
parametrized by closed $C \in C_{1}(\operatorname{Tr}(\mathcal{F}))$ exactly as in the scalar case.

If $C$ is the holonomy invariant current corresponding to the transverse volume form $d t$, then we obtain the Godbillon Vey cyclic cocycle gv on $C_{c}^{\infty}(H(\mathcal{F}), E)$ constructed in [41].

Remark 4.17. The only point in the scalar theory which is not easy to be generalized to general vector bundles is Proposition 4.2.14, since in this case $\left(\Omega_{E}^{0}, \nabla_{Q}\right)$ is no longer a DGA.

\subsubsection{The Godbillon-Vey class: the general case}

In this paragraph we will generalize the construction of the Godbillon-Vey cyclic cocycles done in 4.2 .2 for a transversely oriented compact foliated $\Gamma$-manifold $(M, \mathcal{F}, \Gamma)$ of codimension 1.

For notational simplicity, in this section we will denote simply by $G$ the twisted holonomy groupoid $H(\mathcal{F}, \Gamma)$ of $(M, \mathcal{F}, \Gamma)$. Moreover, we will always assume the group $\Gamma$ to be a compact Lie group.

Let us begin first with an arbitrary not necessarily transversely oriented codimension 1 compact foliated $\Gamma$-manifold with $G=H(\mathcal{F}, \Gamma)$ as twisted holonomy groupoid.

Let us choose once and for all a complete transversal $T$ of $(M, \mathcal{F})$, i.e. a submanifold $T \subseteq M$ of dimension 1 transverse to the foliation which intersects every leaf of $\mathcal{F}$. Since the source map $s$ of $G$ is transverse to $T$, then $G_{T}=s^{-1}(T) \subseteq G$ is a submanifold of $G$. We consider on $G_{T}$ the foliation $\mathcal{F}_{s}$ defined by the surjective submersion $s: G_{T} \rightarrow T$. The leaves of $\mathcal{F}_{s}$ are therefore the connected components of the fibers of $s$. Since $G$ has discrete isotropy groups, the dimension of $\mathcal{F}_{s}$ is equal to $p=\operatorname{dim}\left(G_{x}\right)=\operatorname{dim}(\mathcal{F})$, for all $x \in T$, and its codimension is just $1=\operatorname{dim}(T)$. In particular, one has $\operatorname{dim}\left(G_{T}\right)=\operatorname{dim}(M)=p+1$.

Now, considering the target map $t$ restricted to $G_{T}$, we get

Lemma 4.2.16. 1. The target map $t: G_{T} \rightarrow M$ is a surjective local diffeomorphism. Moreover, viewed as a map between foliated manifolds

$$
t:\left(G_{T}, \mathcal{F}_{s}\right) \rightarrow(M, \mathcal{F})
$$

it is a leafwise map. For all $x \in T$, its restriction to the fiber $G_{x}$

$$
t: G_{x} \rightarrow L_{x}
$$

is a principal bundle onto the leaves $L_{x}$ through $x$ with structure group $G_{x}^{x}$.

2. The pullback foliation $t^{*}(\mathcal{F})$ on $G_{T}$ induced by $t$ coincides with the foliation $\mathcal{F}_{s}$.

Proof. Given any $x \in M$, by definition of the twisted holonomy groupoid the orbit $G x$ of $G$ through $x$ is exactly the leaf $L_{x}$ passing through $x$. Thus, the first item follows from [39], Proposition 5.20 and Theorem 5.4.

The second item is an immediate consequence of the definition of pullback foliation and of the fact that $t$ is a leafwise map. 
Summarizing we are considering

$$
\begin{array}{lll}
G_{T} & \stackrel{s}{\rightarrow} & T \\
\downarrow & & \\
M & &
\end{array}
$$

where $s$ and $t$ are, respectively, the restrictions of the source and the target map of $G$ to $G_{T}$ and satisfy the properties stated in Lemma 4.2.16.

We can define a right action of the étale groupoid $G_{T}^{T}$ on $G_{T}$ simply by right multiplication. It is not difficult to check that this groupoid action is proper and free and that the target map induces a diffeomorphism $\bar{t}: G_{T} / G_{T}^{T} \rightarrow M$. Moreover, if $p: G_{T} \rightarrow G_{T} / G_{T}^{T}$ is the quotient map, then one gets that $\bar{t} \circ p=t$. We refer to [39], 20] and the references therein for more details and further remarks about this and groupoid actions in general.

The étale groupoid $G_{T}^{T}$ acts of course also on its space of objects $T$. More precisely, if $g \in G_{T}^{T}$ with $t(g)=y$ the assignment

$$
(y, g) \mapsto s(g)
$$

defines a right action of $G_{T}^{T}$ on $T$. This action can be equivalently described in the following way. Recall that, being $G_{T}^{T}$ étale, each element $g \in G_{T}^{T}$ induces the germ $\left(h_{g}\right)_{s(g)}$ of a local diffeomorphism $h_{g}$ at $s(g)$ defined as follows. If $U \subseteq G_{T}^{T}$ is an open neighborhood of $g$ such that both $s_{\mid U}$ and $t_{\mid U}$ are diffeomorphisms, one has the local diffeomorphism of $T$ given by

$$
h_{g}=t_{\mid U} \circ\left(s_{\mid U}\right)^{-1} \text {. }
$$

Then $\left(h_{g}\right)_{s(g)}$ is by definition the germ at $s(g)$ of $h_{g}$. It is evident that $\left(h_{g}\right)_{s(g)}$ does only depend on $g$.

Given $g \in G_{T}^{T}$, one set

$$
y \cdot g=\left(h_{g}^{-1}\right)_{t(g)}(t(g))
$$

This defines a right action of $G_{T}^{T}$ on $T$ which coincides with that given by 4.62 . The importance of viewing the $G_{T}^{T}$-action on $T$ as being induced by the germs $\left(h_{g}^{-1}\right)_{t(g)}$ relies on the fact that we can induce a $G_{T}^{T}$-action on the tangent bundle $T(T)$ by taking the linear isomorphisms $d\left(h_{g}^{-1}\right)_{t(g)}: T_{t(g)}(T) \rightarrow T_{s(g)}(T)$ for every $g \in G_{T}^{T}$.

With respect to the above $G_{T}^{T}$-actions, it is immediate to see that the map $s: G_{T} \rightarrow T$ is $G_{T}^{T}$-equivariant, i.e. $s(p g)=s(p) g$, for all $p \in G_{T}, g \in G_{T}^{T}$ such that $s(p)=t(g)$.

Now, the pull-back bundle $s^{*}(T(T))$ fits in the short exact sequence of vector bundles over $G_{T}$

$$
0 \rightarrow \operatorname{ker}(d s) \rightarrow T\left(G_{T}\right) \stackrel{d s}{\longrightarrow} s^{*}(T(T)) \rightarrow 0
$$

Since the foliation $\mathcal{F}_{s}$ on $G_{T}$ is defined by the involutive distribution $\operatorname{ker}(d s)$ it follows that $s^{*}(T(T))$ is isomorphic to the normal bundle of the foliation. Moreover, it inherits a nontrivial action of $G_{T}^{T}$, defined by pulling back through $s$ the $G_{T}^{T}$-action on $T(T)$. 
Lemma 4.2.17. 1. There exists an action of $G_{T}^{T}$ on $T\left(G_{T}\right)$ and on $k e r(d s)$ which turns the sequence 4.63 into a short exact sequence of $G_{T}^{T}$-equivariant vector bundles and $G_{T}^{T}$-equivariant morphisms.

2. The quotient $s^{*}(T(T)) / G_{T}^{T}$ defines a vector bundle over $M$ isomorphic to the normal bundle $\nu$ of the foliation $\mathcal{F}$ of $M$.

3. There exists a $G_{T}^{T}$-invariant subbundle $Q \subseteq T\left(G_{T}\right)$ that is equivariantly isomorphic to $s^{*}(T(T))$.

Proof. 1). Since $G_{T}$ is a proper and free $G_{T}^{T}$-manifold, any element $g \in G_{T}^{T}$ with $s(g)=x$ and $t(g)=y$ induces a diffeomorphism $\alpha_{g}: G_{y} \rightarrow G_{x}$ given by the action. Hence, by taking the differential of these diffeomorphisms one gets a welldefined action of $G_{T}^{T}$ on the tangent bundle to the foliation $T\left(\mathcal{F}_{s}\right)=\operatorname{Ker}(d s)$ over $G_{T}$.

Next, for any $g \in G_{T}^{T}$ and $p \in G_{T}$ with $s(p)=t(g)$, one has a local diffeomorphism induced by $g$ and defined in a suitable neighborhood of $p$. More precisely, take a foliated chart $W \simeq P \times V$ around $p$ with $V \subseteq T$ an open coordinate neighborhood of $s(p) \in T$. Assume further that $W$ and $V$ are chosen in such a way that there is an open set $U \subseteq G_{T}^{T}$ containing $g$, where $t_{\mid U}$ and $s_{\mid U}$ are both diffeomorphisms on their images and $t_{\mid U}(U)=V$. By construction $h_{g}^{-1}=\left(s_{\mid U}\right) \circ\left(t_{\mid U}\right)^{-1}: V \rightarrow V^{\prime}$, where $V^{\prime}=s_{\mid U}(U)$.

Thus, we define $\psi_{g}: W \rightarrow G_{T}$ as follows. For any $q \in W$

$$
\psi_{g}(q)=\alpha_{g_{1}}(q)
$$

where $g_{1} \in U$ is the unique element of $G_{T}^{T}$ whose target is $t\left(g_{1}\right)=s(q)=t^{\prime} \in V$. By construction, the image of $\psi_{g}$ is a foliated chart $W^{\prime} \simeq P \times V^{\prime}$ around $\alpha_{g}(p)=p g$ and $\psi_{g}$ determines a local diffeomorphism of $G_{T}$ which preserves the foliation.

It is readily checked that the germ $\left(\psi_{g}\right)_{p}$ of $\psi_{g}$ at $p$ does only depend on the $p \in G_{T}$ and $g \in G_{T}^{T}$, being independent from the other data needed to define it. Now, for all $p \in G_{T}$ and $g \in G_{T}^{T}$ with $s(p)=t(g)$ we set

$$
\beta(g): T_{p}\left(G_{T}\right) \rightarrow T_{p g}\left(G_{T}\right), \quad \beta(g)=d\left(\psi_{g}\right)_{p} .
$$

This defines a linear action of $G_{T}^{T}$ on $T\left(G_{T}\right)$ turning it into a $G_{T}^{T}$-equivariant vector bundle over $G_{T}$.

Moreover, it is a direct consequence of the definition of the diffeomorphisms $\psi_{g}$ that the bundle morphisms in 4.63 are $G_{T}^{T}$-equivariant, making the sequence an exact sequence of $G_{T}^{T}$-equivariant bundles.

2). Since the action of $G_{T}^{T}$ on $G_{T}$ is proper and free, then the linear $G_{T}^{T}$-action on every equivariant vector bundle over $G_{T}$ is free and proper as well. Thus, the quotient $s^{*}(T(T)) / G_{T}^{T}$ is a manifold equipped with a submersion $\pi: s^{*}(T(T)) / G_{T}^{T} \rightarrow M$ (cf. [39, Lemma 5.35). Moreover, since the target map (that can be identified with the projection map) $t: G_{T} \rightarrow M$ is a local diffeomorphism, then $s^{*}(T(T)) / G_{T}^{T}$ inherits the structure of vector bundle over 
$M$. We denote this bundle more simply by $\tau$.

The same argument can be applied to the vector bundle $T\left(G_{T}\right)$ and to the involutive distribution $\operatorname{ker}(d s)$ and, using that $t$ is also a leafwise map, we get immediately that $T\left(G_{T}\right) / G_{T}^{T} \cong T M$ and $\operatorname{ker}(d s) / G_{T}^{T} \cong T(\mathcal{F})$.

Now, since in our case any $G_{T}^{T}$-equivariant bundle morphism induces a bundle morphism between the quotient vector bundles, one has the commutative diagram

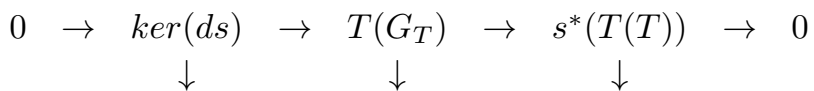

$$
\begin{aligned}
& 0 \rightarrow T(\mathcal{F}) \rightarrow T M \quad \rightarrow \quad \tau \quad \rightarrow 0
\end{aligned}
$$

where the vertical maps are the quotient maps and the horizontal ones in the bottom sequence are those induced by the equivariant morphisms in the top sequence. From this diagram, we deduce that the bottom sequence must be also short exact. This implies that $\tau$ is isomorphic to the normal bundle $\nu$ to the foliation $(M, \mathcal{F})$.

3). Take any distribution $H \subseteq T M$ such that $H \cong \nu$. Then the pullback $Q=$ $t^{*}(H)$ determines a $G_{T}^{T}$-invariant distribution of $T\left(G_{T}\right)$ which is equivariantly isomorphic to $s^{*}(T(T))$, the isomorphism being determined by that between $H$ and $\nu$.

Let us now choose a $G_{T}^{T}$-invariant horizontal distribution $Q \subseteq T\left(G_{T}\right)$ as given by point 3 ) of the lemma above. We know that this amounts to choose a horizontal distribution $H \subseteq T M$ isomorphic to $\nu$.

Let us now suppose that $(M, \mathcal{F}, \Gamma)$ is trasversely oriented and that the induced action of $\Gamma$ on $\nu$ consists of orientation-preserving bundle morphisms. Furthermore, we assume also that the foliation $\mathcal{F}$ is oriented.

We can define a transverse orientation on $G_{T}$ in a such way that the quotient map $Q \rightarrow H$ is orientation-preserving and every element $g \in G_{T}^{T}$ acts on $Q$ as an orientation-preserving linear maps. This orientation induces also an orientation on $T$ such that the bundle morphism $Q \stackrel{d s}{\longrightarrow} T(T)$ becomes orientation-preserving and $G_{T}^{T}$ acts on $T(T)$ through orientation-preserving germs. Moreover, we can induce a leafwise orientation on the foliation $\mathcal{F}_{s}$ of $G_{T}$ making the quotient map and the $G_{T}^{T}$-action orientation-preserving.

Let us choose a volume form $\bar{\Omega}$ of $M$ and consider the pullback $\Omega=t^{*}(\bar{\Omega})$ defined on $G_{T}$. Since $t$ is a local diffeomorphism, $\Omega$ is a $G_{T}^{T}$-equivariant volume form of $G_{T}$. Moreover, if we take a smooth nowhere vanishing section $\bar{\omega} \in C^{\infty}\left(M, \Lambda^{p} T^{*}(\mathcal{F})\right)$, its pullback $\omega=t^{*}(\bar{\omega})$ turns out to be a $G_{T}^{T}$-equivariant nowhere vanishing section on $G_{T}$ of $(k e r(d s))^{*}$, since $t$ is a leafwise map and its restriction to any leaf is a local diffeomorphism (see Lemma 4.2.16).

Let now $\rho^{\prime}$ be a volume form on $T$. It is impossible, in general, to choose this form to be $G_{T}^{T}$-equivariant. Let us consider the section $\rho$ of $Q^{*}$, obtained by taking the pull-back section $s^{*}\left(\rho^{\prime}\right) \in C^{\infty}\left(G_{T}, s^{*}\left(T^{*}(T)\right)\right)$ of $\rho^{\prime}$ along $s$ and then identifying $s^{*}\left(T^{*}(T)\right)$ with $Q^{*}$. 
Since $s$ is a surjective submersion, $\rho$ is nowhere vanishing and, therefore, a defining 1 -form for the foliation $\mathcal{F}_{s}$ on $G_{T}$.

Now, let us consider the smooth positive function $\psi \in C^{\infty}\left(G_{T}\right)$ defined as the Radon-Nikodym derivative

$$
\psi=\frac{\omega \wedge \rho}{\Omega} .
$$

The positive function $\psi \in C^{\infty}\left(G_{T}\right)$ is called a modular function of the groupoid $G$.

Remark 4.18. The choice of the volume form $\rho^{\prime}$ on $T$ determines a positive function $\delta: G_{T}^{T} \rightarrow \mathbb{R}_{+}$on the étale groupoid $G_{T}^{T}$ defined by

$$
\delta=\frac{s^{*}\left(\rho^{\prime}\right)}{t^{*}\left(\rho^{\prime}\right)} .
$$

This in turn defines a one-parameter group $\left(\alpha_{t}\right)_{t \in \mathbb{R}}$ of automorphisms of $C_{r}^{*}\left(G_{T}^{T}\right)$ by the formula

$$
\alpha_{t}(f)(\gamma)=\delta^{-i t}(\gamma) f(\gamma) \quad \forall f \in C_{c}^{\infty}\left(G_{T}^{T}\right), \gamma \in G_{T}^{T}
$$

This one-parameter group leaves $C_{c}^{\infty}\left(G_{T}^{T}\right)$ globally invariant. It can be proved that $\left(\alpha_{t}\right)_{t \in \mathbb{R}}$ is the modular group associated to the normal faithful weight naturally associated to $\rho^{\prime}$. For details about this point, we refer to [17], pag. 263 and the references therein.

The modular function $\psi$ defined above is in general neither compactly supported nor $G_{T}^{T}$-invariant, i.e constant along the orbits. Indeed, from a straightforward computation it follows that $\psi$ is related to the function $\delta \in C^{\infty}\left(G_{T}^{T}\right)$ by the following formula

$$
\psi(p g)=\delta(g) \psi(p)
$$

for any $p \in G_{T}, g \in G_{T}^{T}$ such that $s(p)=t(g)$.

By means of $\psi$, we will now define a one-parameter group of automorphisms of the groupoid $C^{*}$-algebra $C_{r}^{*}(G, E)$ that leaves the smooth convolution algebra $C_{c}^{\infty}(G, E)$ invariant.

Let $E$ be a Hermitian vector bundle over $M$ and let $\tilde{E}=t^{*}(E)$ denote the $G_{T}^{T}$-equivariant Hermitian vector bundle pulled back over $G_{T}$. Consider the space $C_{c}^{\infty}\left(G_{T}, \tilde{E}\right)$. For $t \in \mathbb{R}$ define a linear operator $\Delta^{i t}$ on $C_{c}^{\infty}\left(G_{T}, \tilde{E}\right)$ by setting

$$
\Delta^{i t}(f)=\psi^{-i t} f \quad f \in C_{c}^{\infty}\left(G_{T}, \tilde{E}\right) .
$$

It is well known that using the right $G_{T}^{T}$-action on $G_{T}$, one can $\operatorname{turn} C_{c}^{\infty}\left(G_{T}, \tilde{E}\right)$ into a Hilbert pre- $C^{*}$-module on $C_{c}^{\infty}\left(G_{T}^{T}\right)$. Its completion in the norm given by the $G_{T}^{T}$-valued scalar product gives rise to a Hilbert $C^{*}$-module denoted by $\mathcal{E}_{M}$. For details about this construction, we refer to 30, or 20] and the references therein.

Lemma 4.2.18. The operator $\Delta^{i t}$ extends to a bounded operator of the Hilbert $C^{*}$-module $\mathcal{E}_{M}$ such that 
1. $\left\langle\Delta^{i t} \xi, \Delta^{i t} \eta\right\rangle=\alpha_{t}(\langle\xi, \eta\rangle), \quad \xi, \eta \in \mathcal{E}_{M}$.

2. $\Delta^{i t}(\xi a)=\left(\Delta^{i t}(\xi)\right) \alpha_{t}(a), \quad \xi \in \mathcal{E}_{M}, a \in C_{r}^{*}\left(G_{T}^{T}\right)$

3. The family $\left(\Delta^{i t}\right)_{t \in \mathbb{R}}$ defines a one-parameter group of bounded operators of $\mathcal{E}_{M}$.

Proof. 1). Let $\xi, \eta \in C_{c}^{\infty}\left(G_{T}, \tilde{E}\right)$. Then using Equation 4.70 one gets, for all $\gamma \in G_{T}^{T}$,

$$
\begin{gathered}
\left\langle\Delta^{i t}(\xi), \Delta^{i t}(\eta)\right\rangle(\gamma)=\left\langle\psi^{-i t} \xi, \psi^{-i t} \eta\right\rangle(\gamma)= \\
=\int_{G_{t(\gamma)}}<\left(\psi^{-i t} \xi\right)(p),\left(\psi^{-i t} \eta\right)(p \gamma)>_{\tilde{E}} d p= \\
=\int_{G_{t(\gamma)}}<\psi^{-i t}(p) \xi(p), \psi^{-i t}(p \gamma) \eta(p \gamma)>_{\tilde{E}} d p= \\
=\int_{G_{t(\gamma)}} \overline{\psi^{-i t}(p)} \delta^{-i t}(\gamma) \psi^{-i t}(p)<\xi(p), \eta(p \gamma)>_{\tilde{E}} d p= \\
=\delta^{-i t}(\gamma) \int_{G_{t(\gamma)}}<\xi(p), \eta(p \gamma)>_{\tilde{E}} d p= \\
=\delta^{-i t}(\gamma)(\langle\xi, \eta\rangle)(\gamma)=\alpha_{t}(\langle\xi, \eta\rangle)(\gamma) .
\end{gathered}
$$

In the above computation we have used the action of $\gamma$ to identify $\tilde{E}_{p \gamma}$ with $\tilde{E}_{p}$. Thus the equality 1$)$ holds on $C_{c}^{\infty}\left(G_{T}, \tilde{E}\right)$. Moreover, this implies that

$$
\begin{gathered}
\sup \left\{\frac{\left\|\Delta^{i t}(\xi)\right\|}{\|\xi\|}, \xi \in C_{c}^{\infty}\left(G_{T}, \tilde{E}\right), \xi \neq 0\right\}= \\
=\sup \left\{\frac{\left\|\alpha_{t}(\langle\xi, \xi\rangle)\right\|^{1 / 2}}{\|\langle\xi, \xi\rangle\|^{1 / 2}}, \xi \in C_{c}^{\infty}\left(G_{T}, \tilde{E}\right), \xi \neq 0\right\}=1 .
\end{gathered}
$$

Thus, $\Delta^{i t}$ extends to a bounded operator on the Banach space $\mathcal{E}_{M}$ and the equality holds on the whole $\mathcal{E}_{M}$.

2) follows from a straightforward computation and by continuity. So does 3 ) as well.

In other words, Lemma 4.2 .18 shows that $\mathcal{E}_{M}$ endowed with the $\mathbb{R}$-action given by $\left(\Delta^{i t}\right)_{t \in \mathbb{R}}$ becomes a $\mathbb{R}$-equivariant Hilbert $C^{*}$-module on the $\mathbb{R}$-algebra $C_{r}^{*}\left(G_{T}^{T}\right)$ with action defined by $\left(\alpha_{t}\right)_{t \in \mathbb{R}}($ cf. Remark 4.18$)$.

Now, if $\mathcal{L}\left(\mathcal{E}_{M}\right)$ denotes the $C^{*}$-algebra of adjuntable morphisms of $\mathcal{E}_{M}$, we have that $\Delta^{i t} P \Delta^{-i t} \in \mathcal{L}\left(\mathcal{E}_{M}\right)$ for any $P \in \mathcal{L}\left(\mathcal{E}_{M}\right)$. Setting $\sigma_{t}(P)=\Delta^{i t} P \Delta^{-i t}$, we have

Proposition 4.2.19. 1. The family $\left(\sigma_{t}\right)_{t \in \mathbb{R}}$ on $\mathcal{L}\left(\mathcal{E}_{M}\right)$ defines a one-parameter group of automorphisms of $\mathcal{L}\left(\mathcal{E}_{M}\right)$.

2. One has $\sigma_{t}\left(\mathcal{K}\left(\mathcal{E}_{M}\right)\right) \subseteq \mathcal{K}\left(\mathcal{E}_{M}\right)$ for all $t \in \mathbb{R}$. Therefore, $\left(\sigma_{t}\right)_{t \in \mathbb{R}}$ restricts to a one-parameter group of automorphisms of the $C^{*}$-algebra of compact operators $\mathcal{K}\left(\mathcal{E}_{M}\right)$. 
Proof. 1). The fact that $\Delta^{i t} P \Delta^{-i t} \in \mathcal{L}\left(\mathcal{E}_{M}\right)$, if $P \in \mathcal{L}\left(\mathcal{E}_{M}\right)$, follows easily from Lemma 4.2.18. In particular, the adjoint of $\Delta^{i t} P \Delta^{-i t}$ is just $\Delta^{i t} P^{*} \Delta^{-i t}$. Moreover, it can be checked that $t \rightarrow \sigma_{t}$ is strongly continuous. Hence it defines a one-parameter group of automorphisms.

2). It holds that

$$
\Delta^{i t} \theta_{\xi, \eta} \Delta^{-i t}=\theta_{\Delta^{i t} \xi, \Delta^{i t} \eta}
$$

where $\xi, \eta \in \mathcal{E}_{M}$ and $\theta_{\xi, \eta}$ is the corresponding "rank one" operator. This implies immediately the second statement.

Corollary 4.2.20. The family $\left(\sigma_{t}\right)_{t \in \mathbb{R}}$ induces a one-parameter group of automorphisms of $C_{r}^{*}(G, E)$.

Proof. Since $G$ is a foliation groupoid, the manifold $G_{T}$ is a $\left(G, G_{T}^{T}\right)$-Morita equivalence and therefore $C_{r}^{*}(G, E) \cong \mathcal{K}\left(\mathcal{E}_{M}\right)$. See [20] for details.

Any compactly smoothing operator $K=\left(K_{x}\right)_{x \in M} \in \Psi_{c}^{-\infty}(G, E)$ can be "restricted" to the complete transversal $T$, giving rise to a linear operator

$$
K^{\prime}: C_{c}^{\infty}\left(G_{T}, \tilde{E}\right) \rightarrow C_{c}^{\infty}\left(G_{T}, \tilde{E}\right)
$$

where $\tilde{E}=t^{*}(E)$ denotes the pullback of $E$ over $G_{T}$, such that

- $K^{\prime}$ is the family $K_{\mid T}=\left(K_{t}\right)_{t \in T}$,

- $K^{\prime}$ is $G_{T}^{T}$-invariant,

- the distributional kernel of $K^{\prime}$ has $G_{T}^{T}$-compact support.

Conversely, let $K^{\prime}=\left(K_{t}^{\prime}\right)_{t \in T}$ be a $G_{T}^{T}$-invariant family of compactly smoothing operators on $C_{c}^{\infty}\left(G_{T}, \tilde{E}\right)$, with distributional kernel having $G_{T}^{T}$-compact support. Then, using the $G_{T}^{T}$-invariance, one can extend $K^{\prime}$ to a $G$-invariant family $K=\left(K_{x}\right)_{x \in M}$ of smoothing operators parametrized by $M$, defining a unique element of $\Psi_{c}^{-\infty}(G, E)$.

Lemma 4.2.21. The one-parameter group $\left(\sigma_{t}\right)_{t \in \mathbb{R}}$ preserves the dense subalgebra $C_{c}^{\infty}(G, E) \subseteq C_{r}^{*}(G, E)$.

Proof. Using the isomorphism $\Psi_{c}^{-\infty}(G, E) \cong C_{c}^{\infty}(G, E)$, we need to show that for $K \in \Psi_{c}^{-\infty}(G, E)$, then $\Delta^{i t} K \Delta^{-i t} \in \Psi_{c}^{-\infty}(G, E)$.

Viewing $K$ as a $G_{T}^{T}$-invariant family of operators of $C_{c}^{\infty}\left(G_{T}, \tilde{E}\right)$, the only non trivial point to check is the $G_{T}^{T}$-invariance of $\Delta^{i t} K \Delta^{-i t}$. Let $\gamma \in G_{T}^{T}, \gamma: x \rightarrow y$. Then for any $\xi \in C_{c}^{\infty}\left(G_{x}, \tilde{E}\right)$ and $p \in G_{y}$

$$
\begin{gathered}
U_{\gamma}\left(\Delta_{x}^{i t} \xi\right)(p)=U_{\gamma}\left(\psi^{-i t} \xi\right)(p)= \\
=\left(\psi^{-i t} \xi\right)(p \gamma)=\psi^{-i t}(p \gamma) \xi(p \gamma)= \\
=\delta^{-i t}(\gamma) \psi^{-i t}(p)\left(U_{\gamma} \xi\right)(p)=\delta^{-i t}(\gamma)\left(\Delta_{y}^{i t}\left(U_{\gamma} \xi\right)\right)(p)
\end{gathered}
$$

Hence using this and the invariance of $K$, one gets

$$
U_{\gamma}\left(\Delta^{i t} K \Delta^{-i t}\right)_{x}=U_{\gamma} \Delta_{x}^{i t} K_{x} \Delta_{x}^{-i t}=\delta^{-i t}(\gamma) \Delta_{y}^{i t}\left(U_{\gamma} K_{x}\right) \Delta_{x}^{-i t}=
$$




$$
=\delta^{-i t}(\gamma) \Delta_{y}^{i t} K_{y} U_{\gamma} \Delta_{x}^{-i t}=\delta^{-i t}(\gamma) \Delta_{y}^{i t} K_{y} \delta^{i t}(\gamma) \Delta_{y}^{-i t} U_{\gamma}=\left(\Delta^{i t} K \Delta^{-i t}\right)_{y} U_{\gamma} .
$$

This proves the lemma.

Hence, as in the special case of a foliated bundle, we get

Proposition 4.2.22. $\quad$ 1. The infinitesimal generator of $\left(\sigma_{t}\right)_{t \in \mathbb{R}}$ defines an unbounded derivation of $C_{r}^{*}(G, E)$, whose domain contains the smooth convolution algebra $C_{c}^{\infty}(G, E)$ and which is given by

$$
\delta(K)=[\phi, K] \quad K \in C_{c}^{\infty}(G, E),
$$

where $\phi=\log \psi$.

2. $\delta$ determines a derivation of $C_{c}^{\infty}(G, E)$, i.e. for all $K \in C_{c}^{\infty}(G, E)$, $\delta(K) \in C_{c}^{\infty}(G, E)$. Moreover, this derivation is continuous with respect to the LF-topology on $C_{c}^{\infty}(G, E)$.

Proof. Since $\left(\sigma_{t}\right)_{t \in \mathbb{R}}$ defines a one-parameter group of automorphisms of the $C^{*}$-algebra $C_{r}^{*}(G, E)$, then its infinitesimal generator defines a densely defined operator $\delta$ that is a derivation.

Viewing $K \in C_{c}^{\infty}(G, E)$ as a $G_{T}^{T}$-invariant family of smoothing operators parametrized by $T$ it follows from the definition of $\left(\sigma_{t}\right)_{t \in \mathbb{R}}$ and a straightforward computation that $\delta(K)$ exists and it is equal to the commutator $[\phi, K]$, where $\phi=\log \psi$ acts as a pointwise multiplication operator.

Next, one has that $\delta\left(C_{c}^{\infty}(G, E)\right) \subseteq C_{c}^{\infty}(G, E)$. More precisely, it is easy to see that $[\phi, K]$ represents a family of smoothing operators parametrized by $T$. Furthermore, $[\phi, K]$ is $G_{T}^{T}$-invariant. Indeed, for $\gamma \in G_{T}^{T}, \gamma: x \rightarrow y$, notice that Equation 4.70 implies that

$$
\phi(p \gamma)=\log \delta(\gamma)+\phi(p)
$$

for every $p \in G_{y}$. Hence

$$
\begin{gathered}
U_{\gamma}[\phi, K]_{x}=U_{\gamma}\left(\phi K_{x}-K_{x} \phi\right)=U_{\gamma} \phi K_{x}-U_{\gamma} K_{x} \phi= \\
=(\log \delta(\gamma)+\phi) U_{\gamma} K_{x}-K_{y} U_{\gamma} \phi=(\log \delta(\gamma)+\phi) K_{y} U_{\gamma}-K_{y}(\log \delta(\gamma)+\phi) U_{\gamma}= \\
=\phi K_{y} U_{\gamma}-K_{y} \phi U_{\gamma}=[\phi, K]_{y} U_{\gamma} .
\end{gathered}
$$

Thus, $\delta$ determines a derivation of $C_{c}^{\infty}(G, E)$.

To show that $\delta$ is continuous with respect to the LF-topology on $C_{c}^{\infty}(G, E)$, we first observe that $\delta$ preserves the supports, i.e. if $K \in C_{c}^{\infty}(G, E)$ has support contained in the compact subset $C \subset G$, then the support of $\delta(K)$ will be also contained in $C$. Hence, we need to prove that for all compact subset $C \subset G$, $\delta: C^{\infty}(C, E) \rightarrow C^{\infty}(C, E)$ is continuous with respect to the Fréchet topology on $C^{\infty}(C, E)$. But this follows directly from the fact that $\delta$ is the commutator with the smooth function $\phi$. 
Thus, we have introduced the first derivation we need to define the GodbillonVey cyclic cocycles.

To define the other data needed in the construction, we again use the chosen horizontal distribution $Q \subseteq T\left(G_{T}\right)$ over $G_{T}$ complementary to $\mathcal{F}_{s}$. Recall that $Q$ determines a horizontal distribution $H \subseteq T M$ over $M$ through the quotient map $t: G_{T} \rightarrow M$ such that $t^{*}(H)=Q$.

Let $E$ be a vector bundle over $M$. The space $C_{c}^{\infty}\left(G, s^{*}\left(E^{*}\right) \otimes t^{*}\left(E \otimes H^{*}\right)\right)$ carries a natural right action of the convolution algebra $C_{c}^{\infty}(G, E)$ given by

$$
(P K)(\gamma)=\int_{\gamma_{1} \gamma_{2}=\gamma} P\left(\gamma_{1}\right) K\left(\gamma_{2}\right),
$$

for all $P \in C_{c}^{\infty}\left(G, s^{*}\left(E^{*}\right) \otimes t^{*}\left(E \otimes H^{*}\right)\right), K \in C_{c}^{\infty}(G, E)$.

Equivalently, viewing $P \in C_{c}^{\infty}\left(G, s^{*}\left(E^{*}\right) \otimes t^{*}\left(E \otimes H^{*}\right)\right)$ as a $G_{T}^{T}$-invariant family of operators

$$
\left(P_{t}\right)_{t \in T}: C_{c}^{\infty}\left(G_{T}, t^{*}(E)\right) \rightarrow C_{c}^{\infty}\left(G_{T}, t^{*}(E) \otimes Q^{*}\right)
$$

and $K \in C_{c}^{\infty}(G, E)$ as the corresponding $G_{T}^{T}$-invariant family $\left(K_{t}\right)_{t \in T}$ acting on $C_{c}^{\infty}\left(G_{T}, t^{*}(E)\right)$, one immediately deduces that the above action is simply the composition of these operators, i.e. $P K=P \circ K=\left(P_{t} \circ K_{t}\right)_{t \in T}$.

Next, we can endow $C_{c}^{\infty}\left(G, s^{*}\left(E^{*}\right) \otimes t^{*}\left(E \otimes H^{*}\right)\right)$ with a left action of $C_{c}^{\infty}(G, E)$, turning it into a $C_{c}^{\infty}(G, E)$-bimodule.

Recall that $E N D(E)$ denotes the bundle $s^{*}\left(E^{*}\right) \otimes t^{*}(E)$ and that $C_{c}^{\infty}(G, E)=$ $C_{c}^{\infty}(G, E N D(E))$. One has

Proposition 4.2.23. $\quad$ 1. There exists an algebra isomorphism

$$
\iota: C_{c}^{\infty}(G, E) \rightarrow C_{c}^{\infty}\left(G, E N D\left(E \otimes H^{*}\right)\right)
$$

induced by the chosen transverse volume form $\rho=s^{*}\left(\rho^{\prime}\right)$ on $G_{T}$.

2. Given $P \in C_{c}^{\infty}\left(G, s^{*}\left(E^{*}\right) \otimes t^{*}\left(E \otimes H^{*}\right)\right)$ and $K \in C_{c}^{\infty}(G, E)$, the formula

$$
(K P)(\gamma)=\int_{\gamma_{1} \gamma_{2}=\gamma} \iota(K)\left(\gamma_{1}\right) P\left(\gamma_{2}\right)
$$

determines a left action of $C_{c}^{\infty}(G, E)$ on $C_{c}^{\infty}\left(G, s^{*}\left(E^{*}\right) \otimes t^{*}\left(E \otimes H^{*}\right)\right)$ that commutes with the right action defined by 4.73 .

Proof. 1). Let us consider the kernel groupoid of the submersion $s$

$$
G_{T} \times_{T} G_{T}=\left\{\left(\gamma_{1}, \gamma_{2}\right) \in G_{T} \times G_{T} \mid s\left(\gamma_{1}\right)=s\left(\gamma_{2}\right)\right\}
$$

This is a groupoid over $G_{T}$ with target $\hat{t}$ and source $\hat{s}$ given, respectively, by the first and the second projection.

The étale groupoid $G_{T}^{T}$ acts diagonally on $G_{T} \times_{T} G_{T}$ and this action is clearly free and proper. Moreover, one has the natural morphism of Lie groupoids $\pi: G_{T} \times_{T} G_{T} \rightarrow G$ defined by

$$
\left(\gamma_{1}, \gamma_{2}\right) \mapsto \gamma_{1} \gamma_{2}^{-1}
$$


Since the transversal $T$ is complete, the morphism $\pi$ is surjective and induces an isomorphism of Lie groupoids

$$
\bar{\pi}:\left(G_{T} \times_{T} G_{T}\right) / G_{T}^{T} \rightarrow G .
$$

For a proof that $\left(G_{T} \times_{T} G_{T}\right) / G_{T}^{T}$ is indeed a Lie groupoid over $M$ we refer to 39, Lemma 5.9 .

Now, let us consider the transverse volume form $\rho \in C^{\infty}\left(G_{T}, Q^{*}\right)$ defined above and the corresponding dual section $r \in C^{\infty}\left(G_{T}, Q\right)$ such that $\rho(r) \equiv 1$. We define the smooth section $\hat{s}^{*}(r) \otimes \hat{t}^{*}(\rho) \in C^{\infty}\left(G_{T} \times_{T} G_{T}, \hat{s}^{*}(Q) \otimes \hat{t}^{*}\left(Q^{*}\right)\right)$.

By definition, this is a nowhere vanishing section of the bundle $\hat{s}^{*}(Q) \otimes \hat{t}^{*}\left(Q^{*}\right)$ over $G_{T} \times_{T} G_{T}$. Moreover, it is easy to check using 4.72 that this section is $G_{T}^{T}$-invariant, i.e. constant along the orbits, and for all $\left(p_{1}, p\right),\left(p, p_{2}\right) \in G_{T} \times_{T}$ $G_{T}$ it satisfies

$$
\left(\hat{s}^{*}(r) \otimes \hat{t}^{*}(\rho)\right)\left(\left(p_{1}, p\right)\left(p, p_{2}\right)\right)=\left(\hat{s}^{*}(r) \otimes \hat{t}^{*}(\rho)\right)\left(p_{1}, p\right)\left(\hat{s}^{*}(r) \otimes \hat{t}^{*}(\rho)\right)\left(p, p_{2}\right) .
$$

Hence it descends to a well-defined section $\mu \in C^{\infty}\left(G, s^{*}(H) \otimes t^{*}\left(H^{*}\right)\right)$ which is nowhere vanishing and satisfies $\mu\left(\gamma_{1} \gamma_{2}\right)=\mu\left(\gamma_{1}\right) \mu\left(\gamma_{2}\right)$, for all $\gamma_{1}, \gamma_{2} \in G$ with $s\left(\gamma_{1}\right)=t\left(\gamma_{2}\right)$.

Now, given any $K \in C_{c}^{\infty}(G, E)$ we set

$$
\iota(K)=K \otimes \mu \in C_{c}^{\infty}\left(G, E N D\left(E \otimes H^{*}\right)\right) .
$$

Since the bundle $\operatorname{END}\left(H^{*}\right)$ over $G$ has rank 1, it is then immediate to see that $\iota$ defines an algebra isomorphism between the algebras $C_{c}^{\infty}(G, E)$ and $C_{c}^{\infty}\left(G, E N D\left(E \otimes H^{*}\right)\right)$. This concludes the proof of 1$)$.

2 ). It follows from a straightforward computation, since $\iota$ is an algebra isomorphism.

Remark 4.19. If we view $K$ and $P$ as the corresponding $G_{T}^{T}$-invariant families of operators, the definition of the left action 4.75 is nothing but the composition of operators

$$
K P=\iota(K) \circ P=\left(\iota(K)_{t} \circ P_{t}\right)_{t \in T} .
$$

Important Remark 4.1. Repeating the proof of Lemma 4.2 .8 one can show that the section $\mu$ constructed in Lemma 4.2.23 coincides with the holonomy action $\lambda$ of $G$ on the dual bundle $H^{*}$, viewed as a section $\lambda \in C^{\infty}\left(G, s^{*}(H) \otimes\right.$ $\left.t^{*}\left(H^{*}\right)\right)$.

In other words, the graded vector space $C_{c}^{\infty}(G, E) \oplus C_{c}^{\infty}\left(G, s^{*}\left(E^{*}\right) \otimes t^{*}\left(E \otimes H^{*}\right)\right)$, with graded product induced by the bi-module structure 4.75 is nothing but the transverse differential calculus $\Omega_{E}^{*}$ of the groupoid $G$ (cf. Section 4.1.2).

Let $d_{Q}: C^{\infty}\left(G_{T}\right) \rightarrow C^{\infty}\left(G_{T}, Q^{*}\right)$ be the transverse differentiation on $G_{T}$ with respect to the chosen horizontal distribution $Q$ (see Section 4.1.2) and denote by $d_{Q} \phi \in C^{\infty}\left(G_{T}, Q^{*}\right)$ the transverse derivative of $\phi$.

We can regard $d_{Q} \phi$ as a form-valued multiplication operator

$$
d_{Q} \phi: C_{c}^{\infty}\left(G_{T}, t^{*}(E)\right) \rightarrow C_{c}^{\infty}\left(G_{T}, t^{*}(E) \otimes Q^{*}\right)
$$


Now, using the algebra isomorphism $\iota$ of Lemma 4.2.23, we consider the commutator

$$
\delta_{1}(K)=\left[d_{Q} \phi, K\right]=\left(d_{Q} \phi\right) K-\iota(K) d_{Q} \phi \quad \text { for } K \in C_{c}^{\infty}(G, E)
$$

( $K$ is viewed as the corresponding $G_{T}^{T}$-invariant family of smoothing operators and then the commutator is the usual commutator of operators).

Proposition 4.2.24. $\delta_{1}(K)$ defines an element of $C_{c}^{\infty}\left(G, s^{*}\left(E^{*}\right) \otimes t^{*}\left(E \otimes H^{*}\right)\right)$, for all $K \in C_{c}^{\infty}(G, E)$.

Proof. We need to show that $\delta_{1}(K)$ determines a $G_{T}^{T}$-invariant family of smoothing operators parametrized on $T$ from $C_{c}^{\infty}\left(G_{T}, t^{*}(E)\right)$ to $C_{c}^{\infty}\left(G_{T}, t^{*}(E) \otimes Q^{*}\right)$. Viewing $K \in C_{c}^{\infty}(G, E)$ as the corresponding family of operators, one sees immediately that both operators $\left(d_{Q} \phi\right) K$ and $\iota(K) d_{Q} \phi$ define families of smoothing operators on $T$.

Thus, the only thing to show is the $G_{T}^{T}$-invariance of the family of operators $\left[d_{Q} \phi, K\right]=\left(d_{Q} \phi\right) K-\iota(K) d_{Q} \phi$. But, using the transverse volume form $\rho=s^{*}\left(\rho^{\prime}\right) \in C^{\infty}\left(G_{T}, Q^{*}\right)$ and the function $\delta$ on the étale groupoid $G_{T}^{T}$ defined in Remark 4.18, this follows exactly from the same computation as that done in Lemma 4.2.7.

Corollary 4.2.25. The map

$$
\delta_{1}: C_{c}^{\infty}(G, E) \rightarrow C_{c}^{\infty}\left(G, s^{*}\left(E^{*}\right) \otimes t^{*}\left(E \otimes H^{*}\right)\right)
$$

is a bimodule derivation, that is continuous with respect to the LF-topologies on $C_{c}^{\infty}(G, E)$ and on $C_{c}^{\infty}\left(G, s^{*}\left(E^{*}\right) \otimes t^{*}\left(E \otimes H^{*}\right)\right)$.

Proof. The fact that $\delta_{1}$ is a bimodule derivation follows immediately from the discussion above. The continuity can be proved in the same way as we have done for the continuity of $\delta$. In fact, we first notice that $\delta_{1}$ preserves the supports. Hence, one has to show that for any compact set $C \subset G, \delta_{1}: C^{\infty}(C, E) \rightarrow$ $C^{\infty}\left(C, s^{*}\left(E^{*}\right) \otimes t^{*}\left(E \otimes H^{*}\right)\right)$ is a continuous operator between Fréchet spaces. But this follows directly from the fact that, by definition, $\delta_{1}$ is the commutator with the smooth section $d_{Q} \phi$.

Finally for any $P \in C_{c}^{\infty}\left(G, s^{*}\left(E^{*}\right) \otimes t^{*}\left(E \otimes H^{*}\right)\right)$, we set

$$
\delta^{\prime}(P)=[\phi, P]
$$

where again $\phi=\log \psi$. We get the following lemma, whose proof is completely analogous to that of Lemma 4.2 .221 ).

Lemma 4.2.26. $\delta^{\prime}$ determines a continuous linear map

$$
\delta^{\prime}: C_{c}^{\infty}\left(G, s^{*}\left(E^{*}\right) \otimes t^{*}\left(E \otimes H^{*}\right)\right) \rightarrow C_{c}^{\infty}\left(G, s^{*}\left(E^{*}\right) \otimes t^{*}\left(E \otimes H^{*}\right)\right)
$$

such that $\delta_{1}(\delta(K))=\delta^{\prime}\left(\delta_{1}(K)\right)$, for all $K \in C_{c}^{\infty}(G, E)$. 
Exactly as for foliated bundles, the derivations $\delta, \delta^{\prime}$ and $\delta_{1}$ determine two graded derivations $\delta_{\Omega}^{0}$ and $\delta_{\Omega}^{1}$, respectively, of degree 0 and 1 on the graded algebra $\Omega_{E}^{*}=C_{c}^{\infty}(G, E) \oplus C_{c}^{\infty}\left(G, s^{*}\left(E^{*}\right) \otimes t^{*}\left(E \otimes H^{*}\right)\right)$ by means of the same formulas of Remark 4.16 .

Now, from Lemma 4.1 .3 we know that any closed $P_{\Gamma}$-invariant current $C \in$ $C_{1}(\operatorname{Tr}(\mathcal{F}, \Gamma)$ ) (for the notation see Section 4.1.1), determines a bimodule trace

$$
\int_{C}: C_{c}^{\infty}\left(G, s^{*}\left(E^{*}\right) \otimes t^{*}\left(E \otimes H^{*}\right)\right) \rightarrow \mathbb{C},
$$

i.e. a linear map such that

$$
\int_{C} K P=\int_{C} P K
$$

for every $K \in C_{c}^{\infty}(G, E), P \in C_{c}^{\infty}\left(G, s^{*}\left(E^{*}\right) \otimes t^{*}\left(E \otimes H^{*}\right)\right)$.

These traces happen to be closed with respect to the derivations $\delta_{\Omega}^{0}$ and $\delta_{\Omega}^{1}$.

Lemma 4.2.27. It holds that

$$
\int_{C} \delta_{\Omega}^{1}=0
$$

for every closed invariant current $C \in C_{1}(\operatorname{Tr}(\mathcal{F}, \Gamma))$. Similarly, we have that

$$
\int_{C} \delta_{\Omega}^{0}=0
$$

for all closed current $C \in C_{1}(\operatorname{Tr}(\mathcal{F}, \Gamma))$.

Proof. For the first claim it suffices to prove that $\int_{C} \delta_{1}=0$. Recall that for any $K \in C_{c}^{\infty}(G, E)$

$$
\int_{C} \delta_{1}(K)=\left\langle C, \int_{\mathcal{F}} \operatorname{tr}\left(\left[d_{Q} \phi, K\right]_{\mid M}\right)\right\rangle
$$

By definition of integration along the leaves (cf. 4.3) and since $t: G_{T} \rightarrow M$ is a local diffeomorphism, we may assume that the support of $\operatorname{tr}\left(\left[d_{Q} \phi, K\right]_{\mid M}\right)$ is contained in a foliated chart $U$ diffeomorphic to a foliated chart $\tilde{U}$ in $G_{T}$. Then

$$
\int_{\mathcal{F}} \operatorname{tr}\left(\left[d_{Q} \phi, K\right]_{\mid M}\right)=\int_{\tilde{U} / \mathcal{F}_{s}} \operatorname{tr}\left(\left[d_{Q} \phi, K\right](p, p)\right) d p=0 .
$$

This concludes the proof of the first statement.

For the second claim, one may argue in a similar way.

As a consequence of the above results, we get the following

Proposition 4.2.28. 1. Given any closed invariant current $C \in C_{1}(\operatorname{Tr}(\mathcal{F}, \Gamma))$, the bilinear functional

$$
\dot{\tau}_{C}\left(k_{0}, k_{1}\right)=\int_{C} k_{0} \delta_{1}\left(k_{0}\right)
$$

defines a continuous cyclic $1-$ cocycle on $C_{c}^{\infty}(G, E)$. 
2. The cyclic cocycle $\dot{\tau}_{C}$ is invariant under the $\mathbb{R}$-action on $C_{c}^{\infty}(G, E)$ defined by the one-parameter group of automorphisms $\left(\sigma_{t}\right)_{t \in \mathbb{R}}$.

Proof. 1). It follows from a straightforward computation that $\dot{\tau}$ is a cyclic cocycle. The continuity follows from Corollary 4.2.25. Remark 4.1 and from the proof of Theorem 4.1.4.

2). We must show that the limit

$$
\lim _{t \rightarrow 0} \frac{1}{t}\left(\dot{\tau}_{C}\left(\sigma_{t}\left(k_{0}\right), \sigma_{t}\left(k_{1}\right)\right)-\dot{\tau}_{C}\left(k_{0}, k_{1}\right)\right)=0 .
$$

To prove this, one proceeds in exactly the same way as we did in Proposition 4.2.15. We omit the details.

Since $\dot{\tau}_{C}$ is invariant under the automorphisms $\sigma_{t}$, we can define the contraction of $\dot{\tau}_{C}$ by the infinitesimal generator $\delta$ of $\left(\sigma_{t}\right)_{t \in \mathbb{R}}$. Explicitly this is the continuous cyclic 2-cocycle

$$
\operatorname{gv}_{C}\left(k_{0}, k_{1}, k_{2}\right)=\dot{\tau}_{C}\left(\delta\left(k_{2}\right) k_{0}, k_{1}\right)-\dot{\tau}_{C}\left(k_{0} \delta\left(k_{1}\right), k_{2}\right)
$$

for all $k_{0}, k_{1}, k_{2} \in C_{c}^{\infty}(G, E)$ ( cf. [17], pag. 264).

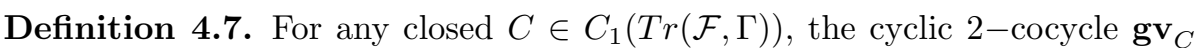
on $C_{c}^{\infty}(G, E)$ is called the distributional $C$-Godbillon-Vey cyclic cocycle of the foliated $\Gamma$-manifold $(M, \mathcal{F}, \Gamma)$ and the bundle $E$.

If $C \in C_{1}(\operatorname{Tr}(\mathcal{F}, \Gamma))$ is the closed invariant current determined by the transverse orientation, then the cyclic cocycle $\mathbf{g v}_{C}$ is a straightforward generalization of the cyclic cocycle constructed in [41] in the case of a foliated bundle.

We point out that all the results of this section should hold more generally for an arbitrary foliation groupoid $G$ integrating the foliated manifold $(M, \mathcal{F})$.

\subsubsection{The equivariant Godbillon-Vey cyclic cocycle}

In this section we will investigate the equivariance of the cyclic cocycles $\mathbf{g v}_{C}$ introduced in the previous section.

Thus, let $(M, \mathcal{F}, \Gamma)$ be a foliated $\Gamma$-manifold which is transversely oriented and of codimension 1 . For simplicity, we further suppose $(M, \mathcal{F})$ to be longitudinally oriented.

Next, let us assume that the induced action of $\Gamma$ on the normal bundle $\nu$ of the foliation consists of orientation-preserving diffeomorphisms.

We consider the twisted holonomy groupoid $H(\mathcal{F}, \Gamma)$ of $(M, \mathcal{F}, \Gamma)$. As in the previous paragraph, for notational simplicity we will denote it simply by $G$.

By Theorem 1.5.7 there exists a group homomorphism $\sigma: \Gamma \rightarrow B i s(G)$, in the group $\operatorname{Bis}(G)$ of global smooth bisections of $G$, such that for all $x \in M$ and $h \in \Gamma, \sigma_{h}(x)$ is the element of $G$ that acts as the germ of the transverse diffeomorphism determined by $h$ in a transverse neighborhood of $x$. See Chapter 1 for details.

If $E$ is a $\Gamma$-equivariant vector bundle over $M$, recall that on $C_{c}^{\infty}(G, E)$ we 
have the action of $\Gamma$ induced by that on $(M, \mathcal{F}, \Gamma)$, determined (cf. Proposition 2.1.15) by the multipliers $S(h)$, which are in turn determined by the bisections $\sigma_{h}$ for every $h \in \Gamma$. We refer to 2.1 .3 for details.

Furthermore, on $C_{c}^{\infty}(G, E)$ we also have the $\mathbb{R}$-action implemented by the one-parameter group of automorphisms $\left(\sigma_{t}\right)_{t \in \mathbb{R}}$, as constructed in the previous section.

We shall define a suitable modular function $\psi$ of the twisted holonomy groupoid $G$ such that the corresponding $\mathbb{R}$-action determined by $\psi$ and $\Gamma$-action will be compatible.

To begin with, let us choose a complete transversal submanifold $T$ of $(M, \mathcal{F}, \Gamma)$. Using the bisections $\sigma_{h}$, for $h \in \Gamma$, we can define on the manifold $G_{T}$ a left action of $\Gamma$ by diffeomorphisms, setting

$$
h \cdot \gamma=\sigma_{h}(t(\gamma)) \gamma \quad h \in \Gamma, \gamma \in G_{T}
$$

Since $\sigma$ is a group homomorphism, it is not difficult to check that this is indeed an action which leaves invariant the source of the points. In other words, each element of $\Gamma$ acts on $G_{T}$ taking each fiber of $s: G_{T} \rightarrow T$ to itself. Therefore, it remains induced an action of $\Gamma$ on $T$, which is trivial. Moreover, this action commutes with the right action of $G_{T}^{T}$ on $G_{T}$ and the target map $t: G_{T} \rightarrow M$ is equivariant with respect to it and the $\Gamma$-action on $M$. Hence, the diagram

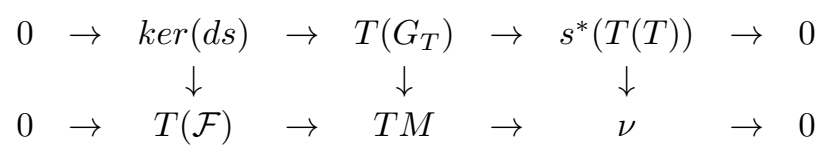

consists of $\Gamma$-equivariant vector bundles and $\Gamma$-equivariant bundle morphisms, with the horizontal sequences short exact.

Since the group $\Gamma$ is compact, we can choose a $\Gamma$-invariant horizontal distribution $H \subseteq T M$ that is equivariantly isomorphic to the normal bundle $\nu$ of the foliation $\mathcal{F}$. Then, the pull-back $Q=t^{*}(H) \subseteq T\left(G_{T}\right)$ of $H$ through the local diffeomorphism $t: G_{T} \rightarrow M$ determines a $\Gamma$-invariant horizontal distribution, which is $\Gamma$-equivariantly isomorphic to $s^{*}(T(T))$.

Remark 4.20. With the above $\Gamma$-action on $G_{T}$ the operator $S(h)$, defined in Proposition 2.1.14, induces by restriction an operator, denoted with the same symbol,

$$
S(h)=\left(S(h)_{t}\right)_{t \in T}: L_{\tau}^{2}\left(G_{T}, t^{*}(E)\right) \rightarrow L_{\tau}^{2}\left(G_{T}, t^{*}(E)\right)
$$

where $S(h)_{t}$ is defined by the formula 2.17 for every $t \in T$.

Now, again because the group $\Gamma$ is compact, we choose a $\Gamma$-invariant volume form $\hat{\Omega}$ on $M$. Then its pullback $\Omega$ on $G_{T}$ through $t$ is also $\Gamma$-invariant, because the target map is equivariant. Moreover, we can also choose a $\Gamma$-invariant nowhere vanishing form $\hat{\omega}_{\mathcal{F}} \in C^{\infty}\left(M, \Lambda^{\operatorname{dim} \mathcal{F}} T^{*} \mathcal{F}\right)$, whose pullback $\omega$ defines a $\Gamma$-invariant leafwise volume form, once again because $t$ is equivariant. Finally, take a volume form $\rho^{\prime}$ on $T$ and consider the corresponding section $\rho=s^{*}\left(\rho^{\prime}\right)$ of $Q^{*}$, as defined in Section 4.2.3. Then, $\rho$ is $\Gamma$-invariant, since the action of $\Gamma$ 
on $G_{T}$ takes each fiber to itself.

Defining the modular function $\psi$ as the Radon-Nykodim derivative

$$
\psi=\frac{\omega \wedge \rho}{\Omega}
$$

we get, therefore, that $\psi$ is a $\Gamma$-invariant modular function. We consider the one-parameter group $\left(\sigma_{t}\right)_{t \in \mathbb{R}}$ defined by $\psi$.

With these choices, we are able to state the compatibility relation between the $\mathbb{R}$-action and the $\Gamma$-action.

Lemma 4.2.29. For every $h \in \Gamma$ and $t \in \mathbb{R}$, it holds that

$$
\begin{gathered}
\sigma_{t}(S(h) \circ K)=S(h) \circ \sigma_{t}(K) \\
\sigma_{t}(K \circ S(h))=\sigma_{t}(K) \circ S(h),
\end{gathered}
$$

for all $K \in C_{c}^{\infty}(G, E)$.

In particular, the $\mathbb{R}$-action and the $\Gamma$-action on $C_{c}^{\infty}(G, E)$ commute.

Proof. We assume first that $E$ is (equivariantly) trivial of rank 1. Given a function $s \in C^{\infty}\left(G_{T}\right)$, the action of an element $h \in \Gamma$ on $s$ is given by the formula $(h \cdot s)(v)=s\left(\sigma_{h^{-1}}(t(v)) v\right)$, for all $v \in G_{T}$.

Now, if $\xi \in C_{c}^{\infty}\left(G_{T}\right)$ and for any $v \in G_{T}$ one has

$$
\begin{gathered}
\left(\Delta^{i t}(S(h) \xi)\right)(v)=\left(\psi^{-i t}(S(h) \xi)\right)(v)= \\
=\psi^{-i t}(v)(S(h) \xi)(v)=\psi^{-i t}(v) \xi\left(\sigma_{h^{-1}}(t(v)) v\right) .
\end{gathered}
$$

Since $\psi$ is $\Gamma$-invariant, we deduce that

$$
\begin{gathered}
\psi^{-i t}(v) \xi\left(\sigma_{h^{-1}}(t(v)) v\right)=\psi^{-i t}\left(\sigma_{h^{-1}}(t(v)) v\right) \xi\left(\sigma_{h^{-1}}(t(v)) v\right)= \\
=\left(\psi^{-i t} \xi\right)\left(\sigma_{h^{-1}}(t(v)) v\right)=\left(S(h)\left(\Delta^{i t} \xi\right)\right)(v) .
\end{gathered}
$$

Then, it follows that $\Delta^{i t}(S(h) \xi)=S(h)\left(\Delta^{i t} \xi\right)$. In other words, since $\xi$ is arbitrary, we have established that $\Delta^{i t} \circ S(h)=S(h) \circ \Delta^{i t}$.

Now, using the above relation let us show that $\sigma_{t}(S(h) \circ K)=S(h) \circ \sigma_{t}(K)$ for all $K \in C_{c}^{\infty}(G)$.

We have

$$
\sigma_{t}(S(h) \circ K)=\Delta^{i t}(S(h) \circ K) \Delta^{-i t}=S(h) \circ\left(\Delta^{i t} K \Delta^{-i t}\right)=S(h) \circ \sigma_{t}(K) .
$$

The other formula is proved similarly.

Now, it is immediate to see that the same argument applies for any equivariant bundle $E$, giving the result.

Finally, that the two actions commute follows from Proposition 2.1.15 and from the equalities we have just proved.

The equivariance of the distributional Godbillon-Vey cyclic cocycles will follow from the following result. 
Proposition 4.2.30. Let $(M, \mathcal{F}, \Gamma)$ be a foliated $\Gamma$-manifold as considered above. Let $E$ be a $\Gamma$-equivariant vector bundle over $M$ and assume to have chosen a $\Gamma$-invariant horizontal distribution $H \subseteq T M$. Denote by $Q \subseteq T\left(G_{T}\right)$ the pullback $t^{*}(H)$ of $H$ through the map $t: G_{T} \rightarrow M$.

Then, for any closed $P_{\Gamma}$-invariant current $C \in C_{1}(\operatorname{Tr}(\mathcal{F}, \Gamma))$ the cyclic 1 -cocycle $\dot{\tau}_{C}$ on $C_{c}^{\infty}(G, E)$ satisfies the Relation 4.1.7, namely

$$
\dot{\tau}_{C}\left(k_{0}, S(h) \circ k_{1}\right)=\dot{\tau}_{C}\left(k_{0} \circ S(h), k_{1}\right),
$$

for every $k_{0}, k_{1} \in C_{c}^{\infty}(G, E), h \in \Gamma$.

Proof. Recall that for all $k_{0}, k_{1} \in C_{c}^{\infty}(G, E)$

$$
\dot{\tau}_{C}\left(k_{0}, k_{1}\right)=\int_{C} k_{0} \delta_{1}\left(k_{1}\right),
$$

where $\delta_{1}(\cdot)=\left[d_{Q} \phi, \cdot\right]$.

Since $Q$ is a $\Gamma$-invariant rank 1 distribution on $G_{T}$ and the function $\phi=\log \psi$ is $\Gamma$-invariant, it follows that the transverse $1-$ form $d_{Q} \phi$ is $\Gamma$-invariant as well, i.e. $h \cdot d_{Q} \phi=d_{Q} \phi$, for every $h \in \Gamma$. Hence, we have that

$$
S(h) \circ\left(\left(d_{Q} \phi\right) k\right)=\left(h \cdot d_{Q} \phi\right)(S(h) \circ k)=d_{Q} \phi(S(h) \circ k),
$$

for every $k \in C_{c}^{\infty}(G, E)$ viewed as the corresponding $G_{T}^{T}$-invariant family of smoothing operators acting on $C_{c}^{\infty}\left(G_{T}, t^{*}(E)\right)$. This implies that

$$
\delta_{1}(S(h) \circ k)=S(h) \circ \delta_{1}(k),
$$

for all $k \in C_{c}^{\infty}(G, E)$. Now the conclusion follows from Proposition 4.1.6 1).

Corollary 4.2.31. With the same assumptions and the notation as in Proposition 4.2.30, for any closed $C \in C_{1}(\operatorname{Tr}(\mathcal{F}, \Gamma))$ one has that

$$
\overline{\mathbf{g v}}_{C}^{\Gamma}\left(k_{0}, k_{1}, k_{2} \mid h\right)=\mathbf{g v}_{C}\left(S(h) \circ k_{0}, k_{1}, k_{2}\right)
$$

defines an equivariant cyclic 2 -cocycle on $\left(C_{c}^{\infty}(G, E), \Gamma\right)$.

Proof. This follows immediately from Proposition 4.2.30 Proposition 4.2.28 2) and Lemma 3.3.4.

Recall that for notational simplicity $G$ stands for the twisted holonomy groupoid $H(\mathcal{F}, \Gamma)$.

Definition 4.8. The equivariant cyclic cocycle $\overline{\mathbf{g v}}_{C}^{\Gamma}$ on $\left(C_{c}^{\infty}(H(\mathcal{F}, \Gamma), E), \Gamma\right)$ is called the equivariant $C$-Godbillon-Vey cyclic cocycle of $(M, \mathcal{F}, \Gamma)$ and the bundle $E$.

Evidently, evaluating $\overline{\mathbf{g v}}_{C}^{\Gamma}$ at the identity $e$ of $\Gamma$ one gets the (nonequivariant) $C$-Godbillon-Vey cyclic cocycle $\mathbf{g v}_{C}$. 
Remark 4.21. Arguing as in Remark 4.12, one can define an equivariant extension of $\mathbf{g v}_{C}$ using Remark 1.15 of Chapter 1 and the invariance of equivariant cyclic cohomology under equivariant Morita equivalences.

As observed in Remark 4.12, these two methods should yield the same equivariant cyclic cocycles.

Now, we are able to define the Lefschetz-Godbillon-Vey numbers. Let $P \in$ $\Psi_{c}^{m}(H(\mathcal{F}, \Gamma), E)$ be a $\Gamma$-invariant elliptic pseudodifferential $H(\mathcal{F}, \Gamma)$-operator and let $h \in \Gamma$. We consider the smooth Lefschetz class $L_{\infty}([h], P)$ of the conjugacy class $[h]$ relative to $P$ defined by

$$
L_{\infty}([h], P)=\frac{i n d_{C^{\infty}}^{\Gamma}(P)}{1_{R[\Gamma]}} \in K_{0}^{\Gamma}\left(C_{c}^{\infty}(H(\mathcal{F}, \Gamma), E)\right)_{[h]}
$$

where $K_{0}^{\Gamma}\left(C_{c}^{\infty}(H(\mathcal{F}, \Gamma), E)\right)_{[h]}$ is the module of fractions at the prime ideal $I_{[h]}$ of $R[\Gamma]$ determined by $[h]$ (cf. 2.2.3).

Let $C \in C_{1}(\operatorname{Tr}(\mathcal{F}, \Gamma))$ closed and let $\overline{g v}_{C}^{\Gamma}$ be the corresponding equivariant Godbillon-Vey cyclic cocycle on $\left(C_{c}^{\infty}(H(\mathcal{F}, \Gamma), E), \Gamma\right)$. With the same notation of Proposition 3.4.5, we set

Definition 4.9. The $\overline{\mathbf{g v}}_{C}^{\Gamma}$-Lefschetz number, or $C$-Godbillon-Vey Lefschetz number, on $H(\mathcal{F}, \Gamma)$ of $[h]$ with respect to the $\Gamma$-invariant elliptic operator $P$ is the number

$$
L_{\overline{\mathbf{g v}}_{C}^{\Gamma}}([h], P)=\left\langle L_{\infty}([h], P), \overline{\mathbf{g v}}_{C}^{\Gamma}\right\rangle_{I_{[h]}}
$$

Remark 4.22. Again by Proposition 3.4.5 and by the definition of $L_{\infty}([h], P)$, we have that

$$
L_{\overline{\mathbf{g v}}_{C}^{\Gamma}}([h], P)=\chi_{\overline{\mathbf{g v}}_{C}^{\Gamma}}([h])\left(i n d_{C}^{\Gamma}(P)\right)=\chi_{\overline{\mathbf{g v}}_{C}^{\Gamma}}(h)\left(i n d_{C^{\infty}}^{\Gamma}(P)\right) .
$$

Thus the $C$-Godbillon-Vey Lefschetz number depends directly on the equivariant index class.

Let now $D \in \Psi_{c}^{m}(H(\mathcal{F}), E)$ be a $\Gamma$-invariant elliptic operator and $\mathcal{L}_{\infty}([h], D)$ its corresponding Lefschetz class. We can restrict $\mathbf{g v}_{C}^{\Gamma}$ to the holonomy groupoid $H(\mathcal{F})$.

Then we define the Godbillon-Vey-Lefschetz number on $H(\mathcal{F})$ of $[h]$ with respect to $D$ by

$$
\mathcal{L}_{\mathbf{g v}_{C}^{\Gamma}}([h], D)=\left\langle\mathcal{L}_{\infty}([h], D), \mathbf{g v}_{C}^{\Gamma}\right\rangle_{I_{[h]}}
$$

where $\mathbf{g v}_{C}^{\Gamma}$ denotes the restricition of $\overline{\mathbf{g v}}_{C}^{\Gamma}$ to $H(\mathcal{F})$.

From Proposition 3.4.4 and the discussion after it one deduces that the Lefschetz numbers do not depend on the group $\Gamma$ as far as this latter exists.

Important Remark 4.2. As we have done in 4.1 .4 for the transverse fundamental cyclic cocycle and the other primary cocycles, we may also define twisted Godbillon-Vey cyclic cocycles $\overline{\mathbf{g v}}_{C}^{h}$. These arise naturally if we consider the action of just one leaf-preserving diffeomorphism $f \in \operatorname{Diff}(\mathcal{F})$ or, equivalently, if we allow the group $\Gamma$ to be the infinite cyclic group $\mathbb{Z}$ generated by $f$.

The procedure above permits to define such cocycles in the same way we did 
for equivariant ones (siehe Section 4.1.4).

This observation is interesting for the following reason. Suppose that $f$ is a leafpreserving diffeomorphism belonging to a compact Lie group $W \subseteq \operatorname{Diff}(M, \mathcal{F})$. Although not every element of $W$ takes a leaf into itself, hence we do not have $W$-equivariant cyclic cocyles, we still have $f$-twisted cyclic cocycles $\overline{\mathbf{g v}}_{C}^{f}$ which can be paired with equivariant $K_{0}^{W}$-theory of the holonomy groupoid, giving rise to higher Godbillon-Vey-Lefschetz numbers also in this case.

\subsection{Some examples and further developments}

In this section we shall briefly outline some example and few properties of the Lefschetz invariants introduced above. Moreover, we would like to give an informal outlook on some possible developments and applications of these invariants and, more generally, of equivariant/twisted index theory for foliated manifolds. Let $(M, \mathcal{F}, \Gamma)$ be a compact foliated $\Gamma$-manifold with $\Gamma$ a compact not discrete Lie group and let $H(\mathcal{F}, \Gamma)$ denote its twisted holonomy groupoid. Consider a closed even dimensional invariant current $C \in C_{e v}(\operatorname{Tr}(\mathcal{F}, \Gamma))$ and the corresponding equivariant cyclic cocycle $\bar{\phi}_{C}$ on $\left(C_{c}^{\infty}(H(\mathcal{F}, \Gamma)), \Gamma\right)$. Moreover, let us take a $\Gamma$-invariant elliptic pseudodifferential $H(\mathcal{F}, \Gamma)$-operator $P$ of order $m$. Then the first straightforward property of higher Lefschetz numbers is the following

Proposition 4.3.1. The $\bar{\phi}_{C}$-Lefschetz number $L_{\bar{\phi}_{C}}([h], P)$ is a continuous function on $\Gamma$. Hence, if $e \in \Gamma$ is the identity of $\Gamma$ and $L_{\bar{\phi}_{C}}(e, P) \neq 0$, then there exists a $h \in \Gamma, h \neq$ e such that $L_{\bar{\phi}_{C}}([h], P) \neq 0$.

Proof. We know that $L_{\bar{\phi}_{C}}([h], P)=\chi_{\bar{\phi}_{C}}(h)\left(i n d_{C}^{\Gamma}(P)\right)$. Now, Theorem 3.4.3 states that the function $h \mapsto \chi_{\bar{\phi}_{C}}(h)\left(i n d_{C^{\infty}}^{\Gamma}(P)\right)$ is a central continuous function on $\Gamma$. This proves the first statement.

The second one is an immediate consequence of the continuity.

We have of course a parallel result for the Godbillon-Vey Lefschetz numbers. Let $(M, \mathcal{F}, \Gamma)$ be a codimension 1 transversely oriented foliated $\Gamma$-manifold with $\Gamma$ a compact not discrete Lie group, whose induced action on the normal bundle preserves the orientation. Let $P$ be a $\Gamma$-invariant elliptic operator as above. Then

Proposition 4.3.2. The $\overline{\mathbf{g v}}_{C}$-Lefschetz number $L_{\overline{\mathbf{g v}}_{C}}([h], P)$ is a continuous function on $\Gamma$. If $e \in \Gamma$ is the identity element and $L_{\overline{\mathbf{g v}}_{C}}(e, P) \neq 0$ then there exists a $h \in \Gamma$ such that $L_{\overline{\mathbf{g v}}_{C}}([h], P) \neq 0$.

Remark 4.23. It is immediate to see that the same results hold for $\mathcal{L}_{\phi_{C}}([h], D)$ and $\mathcal{L}_{\mathbf{g v}_{C}^{\Gamma}}([h], D)$ for $D \in \Psi_{c}^{\infty}(H(\mathcal{F}), E)$.

We point out that in 29] it is shown that the measured Lefschetz number depends continuously on the group. However the proof is much more involved and requires heavy analytic tools. Let us now outline some examples. 
Example 4.2. Let $M$ be a closed oriented manifold and let $G$ be a Lie group acting smoothly on $M$. Suppose that this action is foliated, i.e. the dimension of the stabilizer $G_{x}$ is constant, for all $x \in M$. Then, the orbits of $G$ determine a foliation $(M, \mathcal{F})$ of codimension $q=\operatorname{dim} M-\operatorname{dim} G_{x}$. (see [39], pag. 16).

An important class of examples of foliated actions of compact Lie groups on manifolds comes from orbifolds' theory. More precisely, it can be shown ([39], Section 2.4) that to any compact orbifold $Q$ one can associate a closed manifold $M$ endowed with a foliated action of a compact connected Lie group $G$ such that the leaf space of the corresponding foliation is isomorphic to $Q$. Moreover, if the orbifold is orientable, then one gets a transversely orientable foliated manifold. We refer to [39], Section 2.4 for details.

Back to the general case, we can endow the foliated manifold $(M, \mathcal{F})$ with an action of compact Lie group whose elements act by leaf-preserving diffeomorphisms. Indeed, take a compact subgroup $\Gamma$ of $G$. Since by definition the leaves are the $G$-orbits, each element $h \in \Gamma$ can be viewed as a diffeomorphism of $M$ that takes each leaf into itself. Hence, one obtains a foliated $\Gamma$-manifold $(M, \mathcal{F}, \Gamma)$.

Now, given a closed invariant even dimensional current $C \in C_{e v}(\operatorname{Tr}(\mathcal{F}, \Gamma))$ and a $\Gamma$-invariant elliptic pseudodifferential operator $P$, the Lefschetz numbers $L_{\bar{\phi}_{C}}([h], P)$ and $\mathcal{L}_{\phi_{C}}([h], D)$ provide interesting invariants of the foliated manifold and the action of $\Gamma$. In particular, it seems to be worthwhile to study such Lefschetz-type invariants for foliations coming from orbifolds, as explained above, in order to understand more deeply the latter objects.

Example 4.3. Let $\Sigma_{g}$ be a closed Riemann surface of genus $g \geq 2$ and let $\Lambda \rightarrow \mathbb{H}^{2} \rightarrow \Sigma_{g}$ its universal covering. Consider the action of $\Lambda$ on $S^{1}$ by Moebius transformations and define the diagonal action of $\Lambda$ on the product manifold $\mathbb{H}^{2} \times S^{1}$. Notice that this action is free, proper and cocompact.

The foliation $\mathcal{F}_{0}$ on $\mathbb{H}^{2} \times S^{1}$, whose leaves are given by $\mathbb{H}^{2} \times\{t\}$, for every $t \in S^{1}$, is invariant under the action of $\Lambda$. Hence it descends to a well-defined foliation $\mathcal{F}$ on the quotient manifold $M=\frac{\mathbb{H}^{2} \times S^{1}}{\Lambda}$ whose leaves are the images of the leaves of $\mathcal{F}_{0}$ under the quotient map $q: \mathbb{H}^{2} \times S^{1} \rightarrow M$. In other words, the resulting foliated manifold $(M, \mathcal{F})$ is a leafwise and transversely oriented foliated bundle of codimension 1 (see Example 1.3. Chapter 1).

It is well known (cf. [11, Example 1.3.14 )that the Godbillon-Vey class $G V(\mathcal{F})$ of $(M, \mathcal{F})$ is nontrivial.

Next, let us consider the complex projective plane $\mathbb{C} P^{2}$ as the corresponding oriented smooth manifold of real dimension 4. Further, we consider the oriented manifold $M \times \mathbb{C} P^{2}$ and define the submersion $\pi: M \times \mathbb{C} P^{2} \rightarrow M$ by

$$
M \times \mathbb{C} P^{2} \ni(m, z) \mapsto m .
$$

Since $M$ is a foliated manifold, on $M \times \mathbb{C} P^{2}$ we may consider the pullback foliation $\mathcal{F}_{\pi}=\pi^{*}(\mathcal{F})$. This is a codimension 1 transversely oriented foliation, whose leaves are given by the inverse images

$$
\pi^{-1}(L)=L \times \mathbb{C} P^{2}
$$


where $L$ runs through the leaves of the foliation $(M, \mathcal{F})$. Moreover, it is clear that the tangent bundle $T\left(\mathcal{F}_{\pi}\right)$ to the foliation $\mathcal{F}_{\pi}$ has rank 6 and is isomorphic to $T\left(\mathcal{F}_{\pi}\right) \cong T(\mathcal{F}) \oplus T \mathbb{C} P^{2}$. Here $T \mathbb{C} P^{2}$ denotes the real tangent bundle of the smooth manifold $\mathbb{C} P^{2}$.

By the naturality property of the Godbillon-Vey class we have that

$$
G V\left(\mathcal{F}_{\pi}\right)=G V\left(\pi^{*}(\mathcal{F})\right)=\pi^{*}(G V(\mathcal{F})) .
$$

Since $G V(\mathcal{F}) \in H^{3}(M, \mathbb{R})$ is nontrivial, we immediately deduce that $\pi^{*}(G V(\mathcal{F}))$ defines a nontrivial element in $H^{3}\left(M \times \mathbb{C} P^{2}, \mathbb{R}\right)$. Hence $\left(M \times \mathbb{C} P^{2}, \mathcal{F}_{\pi}\right)$ is a foliated manifold with nontrivial Godbillon-Vey class.

Next, it can be easily shown that the $L$-class of the bundle $T\left(\mathcal{F}_{\pi}\right)$ (cf. [36], Section 11, Chapter III for basic definitions) is nontrivial. Indeed, the $L$-class is a suitable polynomial in the Pontryagin classes of $T\left(\mathcal{F}_{\pi}\right)$ and, for dimensional reasons, the only nontrivial Pontryagin classes of $T\left(\mathcal{F}_{\pi}\right)$ are $p_{0}\left(T\left(\mathcal{F}_{\pi}\right)\right)=1$ and $p_{1}\left(T\left(\mathcal{F}_{\pi}\right)\right)$. By construction and by naturality of the Pontryagin classes, one has that

$$
p_{1}\left(T\left(\mathcal{F}_{\pi}\right)\right)=\pi_{2}^{*}\left(p_{1}\left(T \mathbb{C} P^{2}\right)\right),
$$

where $\pi_{2}: M \times \mathbb{C} P^{2} \rightarrow \mathbb{C} P^{2}$ is the projection on $\mathbb{C} P^{2}$. Since $p_{1}\left(T \mathbb{C} P^{2}\right)$ is nontrivial, one deduces that $p_{1}\left(T\left(\mathcal{F}_{\pi}\right)\right)$ is nonzero and, consequently, that $L\left(T\left(\mathcal{F}_{\pi}\right)\right) \in H^{*}\left(M \times \mathbb{C} P^{2}, \mathbb{R}\right)$ is a nontrivial cohomology class.

Using all these facts we get that

$$
\left\langle L\left(T\left(\mathcal{F}_{\pi}\right)\right) \cap G V\left(\mathcal{F}_{\pi}\right),\left[M \times \mathbb{C} P^{2}\right]\right\rangle \neq 0,
$$

where $\left[M \times \mathbb{C} P^{2}\right] \in H_{7}\left(M \times \mathbb{C} P^{2}, \mathbb{R}\right)$ is the fundamental class of $M \times \mathbb{C} P^{2}$ and $\langle\cdot, \cdot\rangle$ is the pairing between cohomology and homology.

Now, recall the natural action of the compact connected Lie group $U(3)$ on $\mathbb{C} P^{2}$. Hence on the foliated manifold $M \times \mathbb{C} P^{2}$ we have an induced action of $U(3)$ by leaf-preserving diffeomorphisms. Indeed, for any $A \in U(3)$ we define the map $\varphi_{A}: M \times \mathbb{C} P^{2} \rightarrow M \times \mathbb{C} P^{2}$ by setting

$$
\varphi_{A}(m, z)=(m, A z)
$$

This clearly defines a diffeomorphism that takes each leaf into itself. Thus, we have constructed a foliated $U(3)$-manifold $\left(M \times \mathbb{C} P^{2}, \mathcal{F}_{\pi}\right)$.

Since $U(3)$ is compact, there exists a Riemannian metric on $M \times \mathbb{C} P^{2}$ with respect to which each element of $U(3)$ determines a leaf-preserving isometry. Hence, using the chosen metric and orientation on $M \times \mathbb{C} P^{2}$ and since the leaves are even dimensional, we can define the leafwise signature operator $D^{\text {sign, }}+=$ $\left(D_{L}^{\text {sign, }+}\right)_{L \in \mathcal{F}_{\pi}}$. This is a leafwise elliptic differential operator which is also $U(3)$-invariant, since the action of $U(3)$ consists of leaf-preserving isometries. Hence, for every $A \in U(3)$ and every closed $C \in C_{1}(\operatorname{Tr}(\mathcal{F}))$ we can define the Godbillon-Vey Lefschetz number $\mathcal{L}_{\mathbf{g v}_{C}^{U(3)}}\left([A], D^{\text {sign, }}+\right)$ of the conjugacy class $[A]$ of $A$ relative to $D^{\text {sign,+ }}$.

If $I$ denotes the identity element of $U(3)$, one has that $\mathcal{L}_{\mathbf{g v}_{C}^{U(3)}}\left(I, D^{\text {sign, }}\right)$ is the 
Godbillon-Vey higher index $i n d_{\mathbf{g v}_{C}}\left(D^{\text {sign,+}}\right)$ of the signature operator. This (nonequivariant) higher index has been computed (see [4], 25]) and one has

$$
\operatorname{ind}_{\mathbf{g v}_{C}}\left(D^{\text {sign, }+}\right)=\left\langle L\left(T\left(\mathcal{F}_{\pi}\right)\right) \cap G V\left(\mathcal{F}_{\pi}\right),\left[M \times \mathbb{C} P^{2}\right]\right\rangle .
$$

Then, we get that $\mathcal{L}_{\mathbf{g v}_{C}^{U(3)}}\left(I, D^{\text {sign,+}}\right)=i n d_{\mathbf{g v}_{C}}\left(D^{\text {sign, }}\right) \neq 0$ and thus we deduce that there exists an element $A \in U(3)$ such that $\mathcal{L}_{\mathbf{g v}_{C}^{U(3)}}\left([A], D^{\text {sign, }+}\right) \neq$ 0 .

Therefore, Godbillon-Vey Lefschetz numbers are not always zero.

Lefschetz-type invariants are usually related to the fixed point sets of the elements in the conjugacy class $[h]$ of $h$. In particular, if a Lefschetz number of $[h]$ is not zero, then the fixed point set of $[h]$ should be non empty.

For the $\phi_{C}$-Lefschetz numbers this result is shown in [7] in the case of a compact Lie group acting by holonomy diffeomorphisms. Recall that $M^{[h]} \subseteq M$ denotes the fixed point set of the conjugacy class $[h]$ of $h$. Then

Theorem 4.3.3. ([7]) . Let $(M, \mathcal{F}, \Gamma)$ be a closed foliated $\Gamma$-manifold with $\Gamma$ acting by holonomy diffeomorphisms. Let $P \in \Psi_{c}^{m}(H(\mathcal{F}), E)$ be a $\Gamma$-invariant elliptic pseudodifferential operator with values in the $\Gamma$-equivariant bundle $E$. If for a closed $C \in C_{e v}(\operatorname{Tr} \mathcal{F})$ one has that $\mathcal{L}_{\phi_{C}}([h], P) \neq 0$, then $M^{[h]} \neq \emptyset$.

This should, more generally, hold for any compact group acting by leafpreserving diffeomorphisms.

Moreover, similar results should hold for the Godbillon-Vey Lefschetz numbers, as well.

In these cases, however, one has first to show that the equivariant cyclic cocycles extend to a holomorphically closed $\Gamma$-equivariant subalgebra $\mathcal{B}$ of the foliation $\Gamma-C^{*}$-algebra $C_{r}^{*}(H(\mathcal{F}, \Gamma), E)$.

The problem of extending equivariant or twisted cyclic cocycles to equivariant holomorphically closed subalgebras $\mathcal{B}$ of $C_{r}^{*}(H(\mathcal{F}, \Gamma))$ in order to get additive map of the equivariant $K$-theory of the $\Gamma-C^{*}$-algebra $C_{r}^{*}(H(\mathcal{F}, \Gamma))$ is a deep problem in this theory.

For the nonequivariant cyclic cocycles $\tau_{C}$ and the Godbillon-Vey cocycles $\mathbf{g v}_{C}$ it was solved by Connes in [16]. See also [41] for $\mathbf{g v}_{C}$. In the equivariant case, it is sketched in [7] that in the case of a compact Lie group consisting of holonomy diffeomorphisms the equivariant cyclic cocycles $\phi_{C}$ can be extended to a holomorphically closed $\Gamma$-invariant subalgebra $\mathcal{B}$ of $C_{r}^{*}(H(\mathcal{F}))$.

Anyway, solving the extension problem in case of an arbitrary compact Lie group of leaf-preserving diffeomorphisms and for both the primary equivariant cyclic cocycles $\phi_{C}$ and the equivariant Godbillon-Vey cyclic cocycles $\mathbf{g v}_{C}^{\Gamma}$ is an important step that still has to be done, even if we think it should hold true.

Furthermore, the other fundamental problem in the theory is the computation of the higher Lefschetz invariants.

Under a suitable transversality and regularity assumption of the fixed point sets and in the case of a compact Lie group consisting of holonomy diffeomorphisms, the $\phi_{C}$-Lefschetz numbers for any closed even dimensional $C \in C_{e v}(\operatorname{Tr}(\mathcal{F}))$ 
have been computed in [7, Theorem 5.2 and Theorem 5.3.

The resulting cohomological formulas involve characteristic classes of the fixed point set of the elements of the conjugacy classes $[h]$ and are clear generalizations of the classical fixed point theorems due to Atiyah and Segal (cf. [3]).

Restricting only on measured foliations, we have also the Lefschetz theorem due to Heitsch and Lazarov [28, that generalizes more directly the Atiyah-Bott approach to fixed point Lefschetz formulae.

We are interested in extending these formulas to general leaf-preserving diffeomorphisms that do not necessarily satisfy the holonomy assumption.

Moreover, it seems worthwhile to establish fixed point formulae for the GodbillonVey Lefschetz numbers. In this case we expect to obtain fixed point formulae which involve in some way the Godbillon-Vey class restricted to the fixed point sets.

Let us now conclude with few words about possible applications.

First, it seems to be interesting to prove rigidity results for $\phi_{C}$-Lefschetz numbers and Godbillon-Vey Lefschetz numbers analoguous to those proved in [28] for measured foliations. This would permit to get more insight in the structure of the foliated manifold itself and it would provide useful informations about its group of leafwise diffeomorphisms.

As further application, one could use fixed point formulae to detect nontrivial elements of the equivariant $K$-theory group of the foliation $C^{*}$-algebra and, therefore, of the $K$-theory of the crossed product algebra. Indeed, in the nonequivariant theory nontrivial elements and torsion elements of the $K$-theory of the foliation $C^{*}$-algebra can be detected by applying higher index formulae. See, for instance, [16] or [41] for interesting results in this direction.

Hence, it seems natural to use Lefschetz fixed point formulae for detecting nontrivial elements of equivariant $K$-theory of the foliation $C^{*}$-algebra, in order to shed more light on its structure. Finally, since equivariant $K$-theory of the foliation $C^{*}$-algebra with respect to a compact group is isomorphic to the $K$-theory of the corresponding crossed product algebra (cf. 9], Theorem 11.7.1), we would obtain, in this way, informations about the structure of the $K$-theory of the crossed product algebra. 


\section{Appendix A}

\section{Proof of Lemma 4.1.5}

In this appendix we give a proof of the technical lemma, needed in the proof of Theorem 4.1.4. The notation is the same as that of Section 4.1.2. Moreover, for the sake of simplicity we assume the bundle $E$ to be trivial and to have fixed a smooth everywhere positive leafwise density $\alpha$. Thus, we consider functions, rather than densities, and the integrals are computed with respect to the Haar system induced by $\alpha$. Finally, we denote the groupoid $H(\mathcal{F}, \Gamma)$ simply by $G$.

Recall that on the spaces $\Omega^{k}$, for $k=0, \ldots, q$, we have two natural topologies compatible with the vector space structure. The first one is the LF-topology, which turns each $\Omega^{k}$ into a LF-space (cf. 46], pag. 15 and the references therein, or [10, Chapter II, Section 6). If we want to emphasize it, we will denote $\Omega^{k}$ endowed with this topology by the symbol $\Omega_{L F}^{k}$.

The other topology is the so-called compact-open $C^{\infty}$ topology. For the definition of this topology, we refer to [31, Chapter 2 . In this case, if $\Omega^{k}$ carries the compact-open $C^{\infty}$ topology, we shall denote it by $\Omega_{C O}^{k}$.

Lemma A.0.4. Fix $k \leq q$, where $\operatorname{codim} \mathcal{F}=q$, and consider the space $\Omega_{C O}^{k}$. Then, for any $m$-tuple $\left(k_{1}, k_{2}, \ldots, k_{m}\right) \in \mathbb{N}^{l}$ such that $\sum_{l=0}^{m} k_{l}=k$ the multilinear map

$$
\Omega_{L F}^{k_{1}} \times \Omega_{L F}^{k_{2}} \times \cdots \times \Omega_{L F}^{k_{m}} \rightarrow \Omega_{C O}^{k}
$$

defined by the convolution

$$
\left(a^{1}, a^{2}, \ldots, a^{m}\right) \mapsto a^{1} * a^{2} * \cdots * a^{m}
$$

is continuous.

Proof. For the sake of simplicity, we will consider the case $m=2$.

Since we are dealing with topological vector spaces, we only need to show continuity at 0 . Moreover, we need to check it only for a subbasic family of neighborhoods of 0 in $\Omega_{C O}^{k} \cong C_{c}^{\infty}\left(G, \Lambda^{k} t^{*}\left(\nu^{*}\right)\right)$.

We consider a Hermitian metric on $\nu^{*}$. Moreover, we take a coordinate neighborhood $U \subseteq G$ that trivializes the bundle $\Lambda^{k} t^{*}\left(\nu^{*}\right)$ and consider a compact subset $K \subseteq U$. Further, we choose a positive integer $N$ and a positive real number $\epsilon>0$. 
Then we consider the open neighborhood $\mathcal{U}_{K, N}$ of 0 in $C_{c}^{\infty}\left(G, \Lambda^{k} t^{*}\left(\nu^{*}\right)\right)_{C O}$ given by

$$
\mathcal{U}_{K, N}=\left\{f \in C_{c}^{\infty}\left(G, \Lambda^{k} t^{*}\left(\nu^{*}\right)\right) \mid\left\|\partial^{\alpha} f(\gamma)\right\|<\epsilon \text {, for all } \gamma \in K \text { and }|\alpha| \leq N\right\} .
$$

Here, $\|\cdot\|$ is defined through the Hermitian metric on $\Lambda^{k} t^{*}\left(\nu^{*}\right)$ induced by that on $\nu^{*}$ and $|\alpha|$ denotes the lenght of the multindex $\alpha$.

For $i=1,2$, we must find open neighborhoods $\mathcal{V}_{i} \subseteq \Omega_{L F}^{k_{i}} \cong C_{c}^{\infty}\left(G, \Lambda^{k_{i}} t^{*}\left(\nu^{*}\right)\right)$ of 0 such that for every $a^{i} \in \mathcal{V}_{i}$, the convolution $a^{1} * a^{2} \in \mathcal{U}_{K, N}$.

To this purpose, let us take a countable compact exhaustion of $G$, i.e. a countable family $\mathcal{K}$ of compact subsets $K_{j} \subseteq G$ such that $\emptyset=K_{0} \subset K_{1} \subset \stackrel{\circ}{K_{2}} \subset$ $K_{2} \subset \stackrel{\circ}{K}_{3} \subset K_{3} \subset \cdots \subset G$.

For each $i=1,2$, we consider now a step function $\phi_{i}$ on $G$ defined by $\phi_{i}(\gamma)=c_{j}^{i}$ for all $\gamma \in K_{j} \backslash K_{j-1}$ (recall that by definition $K_{0}=\emptyset$ ), where $c_{j}^{i} \in \mathbb{R}$ and $c_{j+1}^{i}<c_{j}^{i}$, for all $j>0$.

Consider the subset $\mathcal{V}_{i} \subseteq \Omega_{L F}^{k_{i}}(i=1,2)$ of all functions $a^{i} \in \Omega_{L F}^{k_{i}}$ such that $\left\|a^{i}\right\|<\phi_{i}$ on $G$, together with all its derivatives up to order $N$.

It is easy to check that each $\mathcal{V}_{i} \subseteq \Omega_{L F}^{k_{i}}$, for $i=1,2$, is an open neighborhood of 0 with respect to the LF-topology on $\Omega^{k_{i}}$.

Moreover, by choosing the values $c_{j}^{i}$ of the step functions small enough, it can be directly checked that for all $\left(a^{1}, a^{2}\right) \in \mathcal{V}_{1} \times \mathcal{V}_{2}$ one gets $a^{1} * a^{2} \in \mathcal{U}_{K, N}$.

Indeed, let $a^{1} \in \mathcal{V}_{1}, a^{2} \in \mathcal{V}_{2}$ and fix $\gamma \in K$ with $s(\gamma)=x$ and $t(\gamma)=y$. One has

$$
\begin{gathered}
\left\|\left(a^{1} * a^{2}\right)(\gamma)\right\|=\left\|\int_{G^{y}} a^{1}\left(\gamma_{1}\right) \lambda\left(\gamma_{1}\right) a^{2}\left(\gamma_{1}^{-1} \gamma\right) d \alpha^{y}\left(\gamma_{1}\right)\right\| \leq \\
\leq \int_{G^{y}}\left\|a^{1}\left(\gamma_{1}\right)\right\|\left\|\lambda\left(\gamma_{1}\right) a^{2}\left(\gamma_{1}^{-1} \gamma\right)\right\| d \alpha^{y}\left(\gamma_{1}\right) \leq \\
\leq \int_{G^{y}}\left\|a^{1}\left(\gamma_{1}\right)\right\|\left\|\lambda\left(\gamma_{1}\right)\right\|\left\|a^{2}\left(\gamma_{1}^{-1} \gamma\right)\right\| d \alpha^{y}\left(\gamma_{1}\right),
\end{gathered}
$$

where $\left\|\lambda\left(\gamma_{1}\right)\right\|$ denotes the operator norm of $\lambda\left(\gamma_{1}\right): \Lambda^{k_{2}} \nu_{s\left(\gamma_{1}\right)}^{*} \rightarrow \Lambda^{k_{2}} \nu_{t\left(\gamma_{1}\right)}^{*}$ given by infinitesimal holonomy.

Since $a^{1}$ and $a^{2}$ have both compact support, there exists an index $m \in \mathbb{N}$ such that the compact subset $K_{m} \in \mathcal{K}$ contains both supports of $a^{1}$ and $a^{2}$. Then

$$
\begin{aligned}
& \int_{G^{y}}\left\|a^{1}\left(\gamma_{1}\right)\right\|\left\|\lambda\left(\gamma_{1}\right)\right\|\left\|a^{2}\left(\gamma_{1}^{-1} \gamma\right)\right\| d \alpha^{y}\left(\gamma_{1}\right)= \\
= & \int_{G^{y} \cap K_{m}}\left\|a^{1}\left(\gamma_{1}\right)\right\|\left\|\lambda\left(\gamma_{1}\right)\right\|\left\|a^{2}\left(\gamma_{1}^{-1} \gamma\right)\right\| d \alpha^{y}\left(\gamma_{1}\right) .
\end{aligned}
$$

Now, since by hypothesis $a^{2} \in \mathcal{V}_{2}$, there is a constant $c_{0}$ indipendent of $\gamma$ such that

$$
\begin{gathered}
\int_{G^{y} \cap K_{m}}\left\|a^{1}\left(\gamma_{1}\right)\right\|\left\|\lambda\left(\gamma_{1}\right)\right\|\left\|a^{2}\left(\gamma_{1}^{-1} \gamma\right)\right\| d \alpha^{y}\left(\gamma_{1}\right) \leq \\
\leq c_{0} \int_{G^{y} \cap K_{m}}\left\|a^{1}\left(\gamma_{1}\right)\right\|\left\|\lambda\left(\gamma_{1}\right)\right\| d \alpha^{y}\left(\gamma_{1}\right) .
\end{gathered}
$$


Moreover, since the compact subsets $K_{j}$ are nested into each other, one gets that

$$
\begin{gathered}
c_{0} \int_{G^{y} \cap K_{m}}\left\|a^{1}\left(\gamma_{1}\right)\right\|\left\|\lambda\left(\gamma_{1}\right)\right\| d \alpha^{y}\left(\gamma_{1}\right) \leq \\
c_{0} \sum_{j=1}^{m} \int_{G^{y} \cap\left(K_{j} \backslash K_{j-1}\right)}\left\|a^{1}\left(\gamma_{1}\right)\right\|\left\|\lambda\left(\gamma_{1}\right)\right\| d \alpha^{y}\left(\gamma_{1}\right) .
\end{gathered}
$$

Viewing the holonomy action as a smooth section $\lambda: G \rightarrow E N D\left(\nu^{*}\right)$, one easily deduces that for all $j \geq 1$ there exist a constant $N_{j}$ such that $\left\|\lambda\left(\gamma_{1}\right)\right\| \leq N_{j}$ for all $\gamma_{1} \in K_{j} \backslash K_{j-1}$. Moreover, by definition of the open set $\mathcal{V}_{1}$, it follows that $\left\|a^{1}\left(\gamma_{1}\right)\right\|<c_{j}^{1}$ for every $\gamma_{1} \in K_{j} \backslash K_{j-1}$. Then one has that

$$
\begin{gathered}
c_{0} \sum_{j=1}^{m} \int_{G^{y} \cap\left(K_{j} \backslash K_{j-1}\right)}\left\|a^{1}\left(\gamma_{1}\right)\right\|\left\|\lambda\left(\gamma_{1}\right)\right\| d \alpha^{y}\left(\gamma_{1}\right)< \\
<c_{0} \sum_{j=1}^{m} N_{j} c_{j}^{1} \int_{G^{y} \cap\left(K_{j} \backslash K_{j-1}\right)} d \alpha^{y}\left(\gamma_{1}\right) .
\end{gathered}
$$

By the continuity of the Haar system $d \alpha=\left(d \alpha^{x}\right)_{x \in M}$ with respect to $x$ and the fact that $\gamma$ varies in the fixed compact set $K$, it follows immediately that the integral $\int_{G^{y} \cap\left(K_{j} \backslash K_{j-1}\right)} d \alpha^{y}\left(\gamma_{1}\right)$ can be estimated uniformly with respect to $y=t(\gamma)$ by some constant $M_{j}$.

Hence, we get finally that

$$
c_{0} \sum_{j=1}^{m} N_{j} c_{j}^{1} \int_{G^{y} \cap\left(K_{j} \backslash K_{j-1}\right)} d \alpha\left(\gamma_{1}\right)<c_{0} \sum_{j=1}^{m} N_{j} M_{j} c_{j}^{1} .
$$

Now, by choosing for instance $c_{j}^{1}=\frac{\epsilon}{c_{0} M_{j} N_{j}} \frac{1}{2^{j}}$, we immediately deduce that

$$
c_{0} \sum_{j=1}^{m} N_{j} M_{j} c_{j}^{1}<\sum_{j=1}^{\infty} c_{0} N_{j} M_{j} c_{j}^{1} \leq \epsilon .
$$

This shows that $a^{1} * a^{2} \in \mathcal{U}_{K, N}$. A similar computation can be done to estimate the derivatives of $a^{1} * a^{2}$ up to order $N$.

Since the open sets of the form $\mathcal{U}_{K, N}$ generate the CO-topology, we deduce the lemma. 


\section{Bibliography}

[1] I. Androulidakis and G. Skandalis. The holonomy groupoid of a singular foliation. J. Reine Angew. Math., 626:1-37, 2009.

[2] M. Atiyah and I. Macdonald. Introduction to commutative algebra, volume 1. Addison-Wesley Reading, Mass, 1969.

[3] M. Atiyah and G. Segal. The index of elliptic operators: Ii. The Annals of Mathematics, 87(3):531-545, 1968.

[4] M. Atiyah and I. Singer. The index of elliptic operators: I. The Annals of Mathematics, 87(3):484-530, 1968.

[5] M.-T. Benameur. A longitudinal Lefschetz theorem in $K$-theory. $K$-Theory, 12(3):227-257, 1997.

[6] M.-T. Benameur and J. L. Heitsch. "Index theory and non-commutative geometry. I. Higher families index theory" - corrigendum [ $K$-Theory $\mathbf{3 3}$ (2004), no. 2, 151-183; ]. K-Theory, 36(3-4):397-402 (2006), 2005.

[7] M.-T. Benameur and J. L. Heitsch. The higher fixed point theorem for foliations. I. Holonomy invariant currents. J. Funct. Anal., 259(1):131-173, 2010.

[8] M.-T. Benameur and P. Piazza. Index, eta and rho invariants on foliated bundles. Astérisque, (327):201-287 (2010), 2009.

[9] B. Blackadar. K-theory for operator algebras, volume 5 of Mathematical Sciences Research Institute Publications. Cambridge University Press, Cambridge, second edition, 1998.

[10] N. Bourbaki. Topological vector spaces. Chapters 1-5. Elements of Mathematics (Berlin). Springer-Verlag, Berlin, 1987. Translated from the French by H. G. Eggleston and S. Madan.

[11] A. Candel and L. Conlon. Foliations. I, volume 23 of Graduate Studies in Mathematics. American Mathematical Society, Providence, RI, 2000.

[12] A. Candel and L. Conlon. Foliations. II, volume 60 of Graduate Studies in Mathematics. American Mathematical Society, Providence, RI, 2003. 
[13] A. Connes. Sur la théorie non commutative de l'intégration. In Algèbres d'opérateurs (Sém., Les Plans-sur-Bex, 1978), volume 725 of Lecture Notes in Math., pages 19-143. Springer, Berlin, 1979.

[14] A. Connes. A survey of foliations and operator algebras. In Operator algebras and applications, Part I (Kingston, Ont., 1980), volume 38 of Proc. Sympos. Pure Math., pages 521-628. Amer. Math. Soc., Providence, R.I., 1982.

[15] A. Connes. Noncommutative differential geometry. Inst. Hautes Études Sci. Publ. Math., (62):257-360, 1985.

[16] A. Connes. Cyclic cohomology and the transverse fundamental class of a foliation. In Geometric methods in operator algebras (Kyoto, 1983), volume 123 of Pitman Res. Notes Math. Ser., pages 52-144. Longman Sci. Tech., Harlow, 1986.

[17] A. Connes. Noncommutative geometry. Academic Press Inc., San Diego, CA, 1994.

[18] A. Connes, J. Cuntz, E. Guentner, N. Higson, J. Kaminker, and J. E. Roberts. Noncommutative geometry, volume 1831 of Lecture Notes in Mathematics. Springer-Verlag, Berlin, 2004. Lectures given at the C.I.M.E. Summer School held in Martina Franca, September 3-9, 2000, Edited by S. Doplicher and R. Longo, Fondazione C.I.M.E.. [C.I.M.E. Foundation].

[19] M. Crainic and I. Moerdijk. Foliation groupoids and their cyclic homology. Adv. Math., 157(2):177-197, 2001.

[20] C. Daenzer. A groupoid approach to noncommutative T-duality. Comm. Math. Phys., 288(1):55-96, 2009.

[21] C. Debord and J.-M. Lescure. Index theory and groupoids. In Geometric and topological methods for quantum field theory, pages 86-158. Cambridge Univ. Press, Cambridge, 2010.

[22] A. Fermi. An introduction to foliations and groupoids. Quaderni del Seminario Matematico di Brescia, 31, 2011.

[23] D. G. Gong. Excision of equivariant cyclic cohomology of topological algebras. Michigan Math. J., 39(3):455-473, 1992.

[24] A. Gorokhovsky. Explicit formulae for characteristic classes in noncommutative geometry. Ph.D. Thesis, OSU.

[25] A. Gorokhovsky and J. Lott. Local index theory over étale groupoids. J. Reine Angew. Math., 560:151-198, 2003.

[26] A. Gorokhovsky and J. Lott. Local index theory over foliation groupoids. Adv. Math., 204(2):413-447, 2006. 
[27] A. Haefliger. Some remarks on foliations with minimal leaves. J. Differential Geom., 15(2):269-284 (1981), 1980.

[28] J. L. Heitsch and C. Lazarov. A Lefschetz theorem for foliated manifolds. Topology, 29(2):127-162, 1990.

[29] J. L. Heitsch and C. Lazarov. Rigidity theorems for foliations by surfaces and spin manifolds. Michigan Math. J., 38(2):285-297, 1991.

[30] M. Hilsum and G. Skandalis. Stabilité des $C^{*}$-algèbres de feuilletages. Ann. Inst. Fourier (Grenoble), 33(3):201-208, 1983.

[31] M. W. Hirsch. Differential topology, volume 33 of Graduate Texts in Mathematics. Springer-Verlag, New York, 1994. Corrected reprint of the 1976 original.

[32] S. Hurder. Classifying foliations. In Foliations, geometry, and topology, volume 498 of Contemp. Math., pages 1-65. Amer. Math. Soc., Providence, RI, 2009.

[33] S. Hurder and A. Katok. Secondary classes and transverse measure theory of a foliation. Bull. Amer. Math. Soc. (N.S.), 11(2):347-350, 1984.

[34] S. Klimek, W. Kondracki, and A. Lesniewski. Equivariant entire cyclic cohomology. I. Finite groups. K-Theory, 4(3):201-218, 1991.

[35] Y. A. Kordyukov. Noncommutative geometry of foliations. J. K-Theory, 2(2, Special issue in memory of Yurii Petrovich Solovyev. Part 1):219-327, 2008.

[36] H. B. Lawson, Jr. and M.-L. Michelsohn. Spin geometry, volume 38 of Princeton Mathematical Series. Princeton University Press, Princeton, NJ, 1989.

[37] J.-L. Loday. Cyclic homology, volume 301 of Grundlehren der Mathematischen Wissenschaften [Fundamental Principles of Mathematical Sciences]. Springer-Verlag, Berlin, second edition, 1998. Appendix E by María O. Ronco, Chapter 13 by the author in collaboration with Teimuraz Pirashvili.

[38] K. C. H. Mackenzie. General theory of Lie groupoids and Lie algebroids, volume 213 of London Mathematical Society Lecture Note Series. Cambridge University Press, Cambridge, 2005.

[39] I. Moerdijk and J. Mrčun. Introduction to foliations and Lie groupoids, volume 91 of Cambridge Studies in Advanced Mathematics. Cambridge University Press, Cambridge, 2003.

[40] C. C. Moore and C. L. Schochet. Global analysis on foliated spaces, volume 9 of Mathematical Sciences Research Institute Publications. Cambridge University Press, New York, second edition, 2006. 
[41] H. Moriyoshi and T. Natsume. The Godbillon-Vey cyclic cocycle and longitudinal Dirac operators. Pacific J. Math., 172(2):483-539, 1996.

[42] V. Nistor, A. Weinstein, and P. Xu. Pseudodifferential operators on differential groupoids. Pacific J. Math., 189(1):117-152, 1999.

[43] W. Thurston. Noncobordant foliations of $S^{3}$. Bull. Amer. Math. Soc., 78:511-514, 1972.

[44] W. Thurston. The theory of foliations of codimension greater than one. Comment. Math. Helv., 49:214-231, 1974.

[45] S. Vassout. Unbounded pseudodifferential calculus on Lie groupoids. $J$. Funct. Anal., 236(1):161-200, 2006.

[46] C. Voigt. Equivariant cyclic homology. Ph.D. Thesis, University of Münster.

[47] H. E. Winkelnkemper. The graph of a foliation. Ann. Global Anal. Geom., $1(3): 51-75,1983$. 